\title{
Digital Regionalism: Identity, Place, and the Ottawa Train Station
}

\author{
by
}

\section{Benjamin Connolly}

A thesis submitted to the Faculty of Graduate and Post Doctoral Affairs in partial fulfillment of the requirements for the degree of

Master of Architecture

\author{
Carleton University \\ Ottawa, Ontario \\ (C) 2015 \\ Benjamin Connolly
}




\section{$\underline{\text { ABSTRACT }}$}

The Critical Regionalist ethos was born of a staunch distrust to the utopian visions of Internationalism, succeeding in a retreat back to an architecture of local identity. With the onset of the globalized 21st-century architecture practice, digital design tools have subsequently come to homogenize a transnational working methodology where the importance of regional peripheries is receding over time.

This thesis questions the role of digital design and regionalism in contemporary architecture. How can architecture reconcile regional identity drivers with the increasing use of digital design tools for complex projects? Recognizing the role that advanced digital working methods have in contemporary design, this thesis uses a digital tool set to interpret natural wood phenomena that constitutes a novel design system. It expands the analysis to interpolate multidirectional patterns that otherwise go unseen, with the results used to produce a new placeform. In doing so it reinterprets the genius loci of place for a contemporary digital regionalism.

This thesis proposes an amalgamated transit terminal at the Ottawa Train Station as a series of node points dictating movement relationships as the generator of architecture. This project is in anticipation of a bullet-train system coming online in the future through the Quebec City - Windsor corridor, syncing with the ongoing LRT system slated for opening in 2018. 


\section{ACKNOWLEDGMENTS}

Johan Voordouw - for allowing me vast freedom in exploring abstract ideas while offering exceptional advice to keep me on the right track. Completing this thesis owes a huge gratitude to you.

Roly Hudson - for forming my first interests in advanced digital techniques and instilling a rigid approach to design. Your teaching during undergrad had a huge influence on this work.

Chris Kaltenbach - for your guidance in developing a keen eye for clean design and lively critical discourse, especially in bridging material culture with digital design.

Additional thanks to David Hines at Populous for taking the time to discuss the professional side of applying parametric design to built projects and providing a unique perspective to the thesis work.

Most importantly, for Mom + Dad + Jess. 


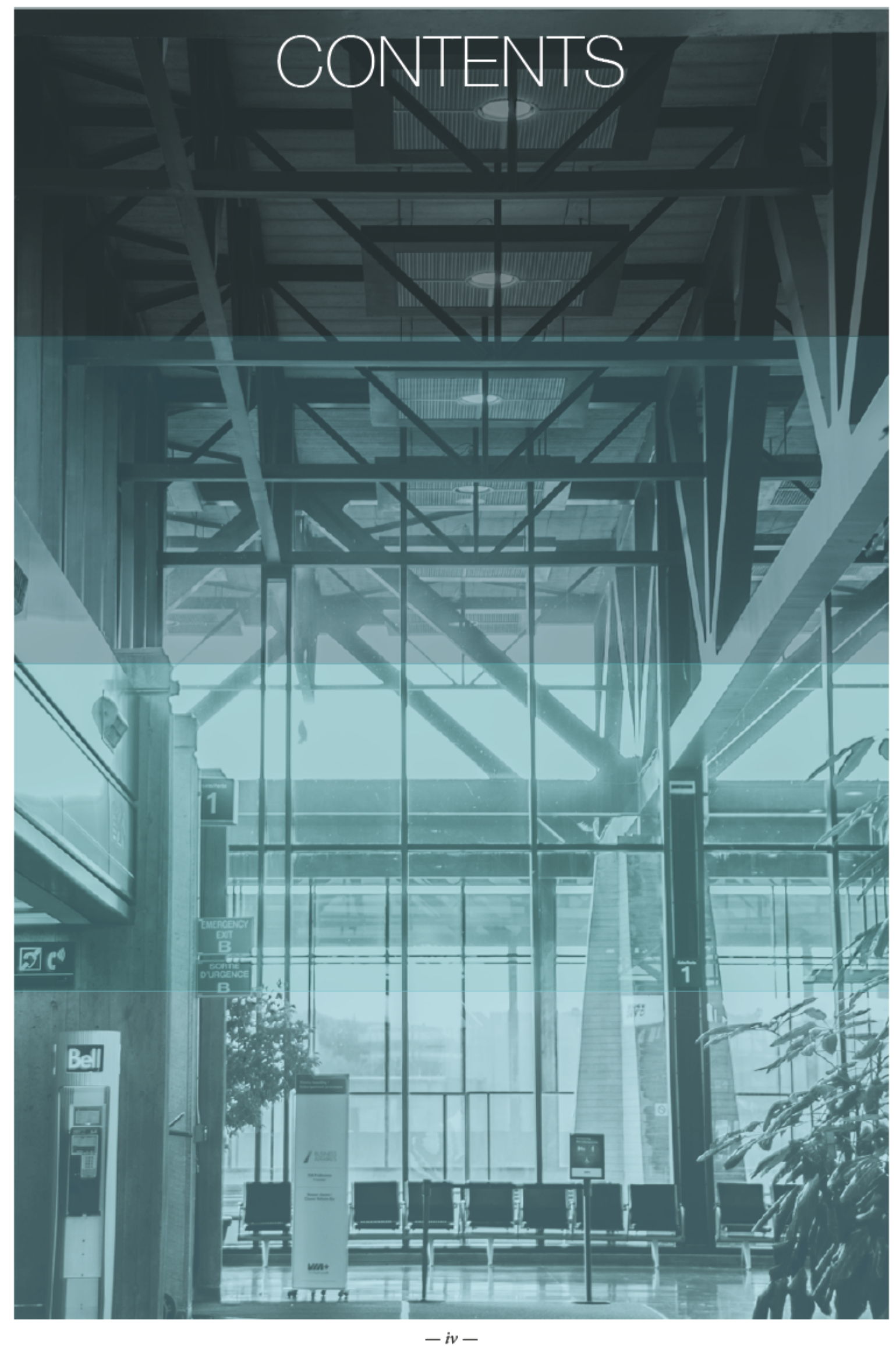


Ch.00 Forward

$\begin{array}{lll}0.1 & \text { Abstract } & \text { ii } \\ 0.2 & \text { Acknowledgments } & \text { iii } \\ 0.3 & \text { Contents } & \text { iv }\end{array}$

Ch.01 The Role of the Digital in Contemporary Discourse 2

1.1 Introduction 5

1.2 Prologue 11

1.3 Post-Medium and the Digital Method 15

1.4 Hidden Truth and False Complexity 17

Ch.02 Globalization as a Re-Emerging Internationalism 25

2.1 Form / Function $\quad 27$

2.2 Digital / Physical 33

2.3 Investigation I: Reconstructive Phenomena 38

Ch.03 Phenomena and the Existing Condition as a Critical Response 49

3.1 Digital Contextualism 51

3.2 Imitative Entities: Biomimicry and Phenomena $\quad 60$

3.3 Investigation II: Predictive Phenomena 63

Ch.04 VIA 1.0: The Ottawa Train Station 79

4.1 High-Speed for Ottawa 91

4.2 Nodes and Node-Based Systems 94

4.3 Investigation III: Sited Phenomena 108

Ch.05 The Problem of Place and Identity in the 21st Century 120

5.1 Transnational Practice 122

5.2 The Tyranny of Place 123

$\begin{array}{ll}5.3 & \text { Plain Contemporary } \\ 5.4 & 127\end{array}$

5.4 Digital Regionalism 130

5.5 Investigation IV: Developed Phenomena 134

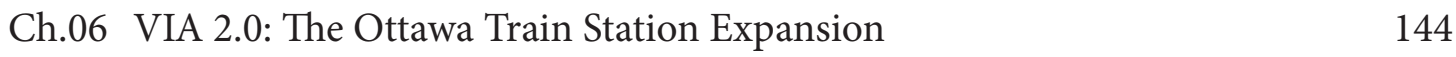

6.1 Proposal 146

$\begin{array}{ll}\text { Ch.07 Conclusion } & 161\end{array}$

$\begin{array}{lll}7.1 & \text { Conclusion } & 163\end{array}$

$\begin{array}{lll}7.2 & \text { Post-script } & 167\end{array}$

$\begin{array}{lll}\text { Appendix A } & \text { Populous: A Conversation with David Hines }\end{array}$

$\begin{array}{lll}\text { Appendix B } & 186\end{array}$ 

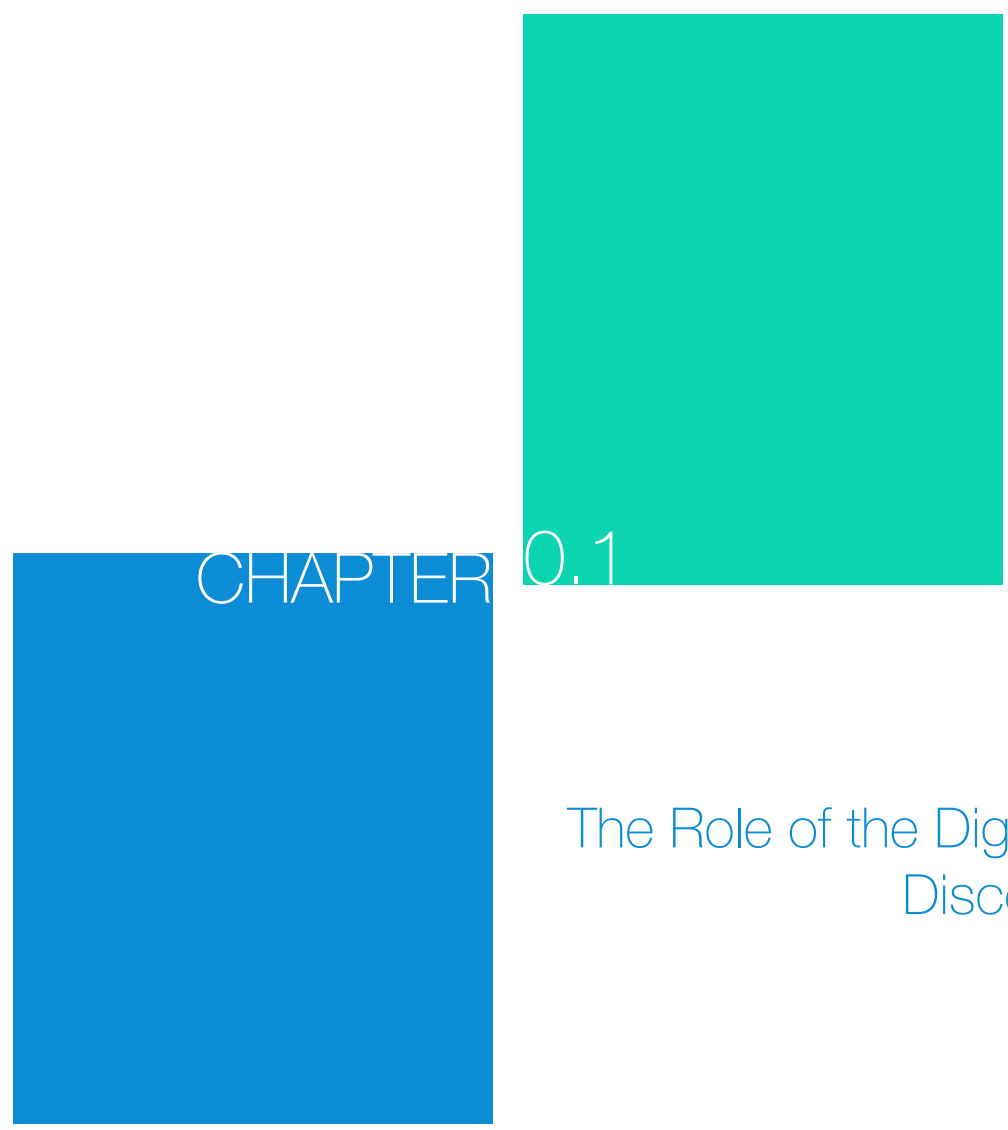

The Role of the Digital in Contemporary Discourse 
"The computer allows you to start a sentence without knowing its continuation. Dealing with the pen or the typewriter, you have to know exactly what you want and where you're heading." _ Thure Erik Lund. 


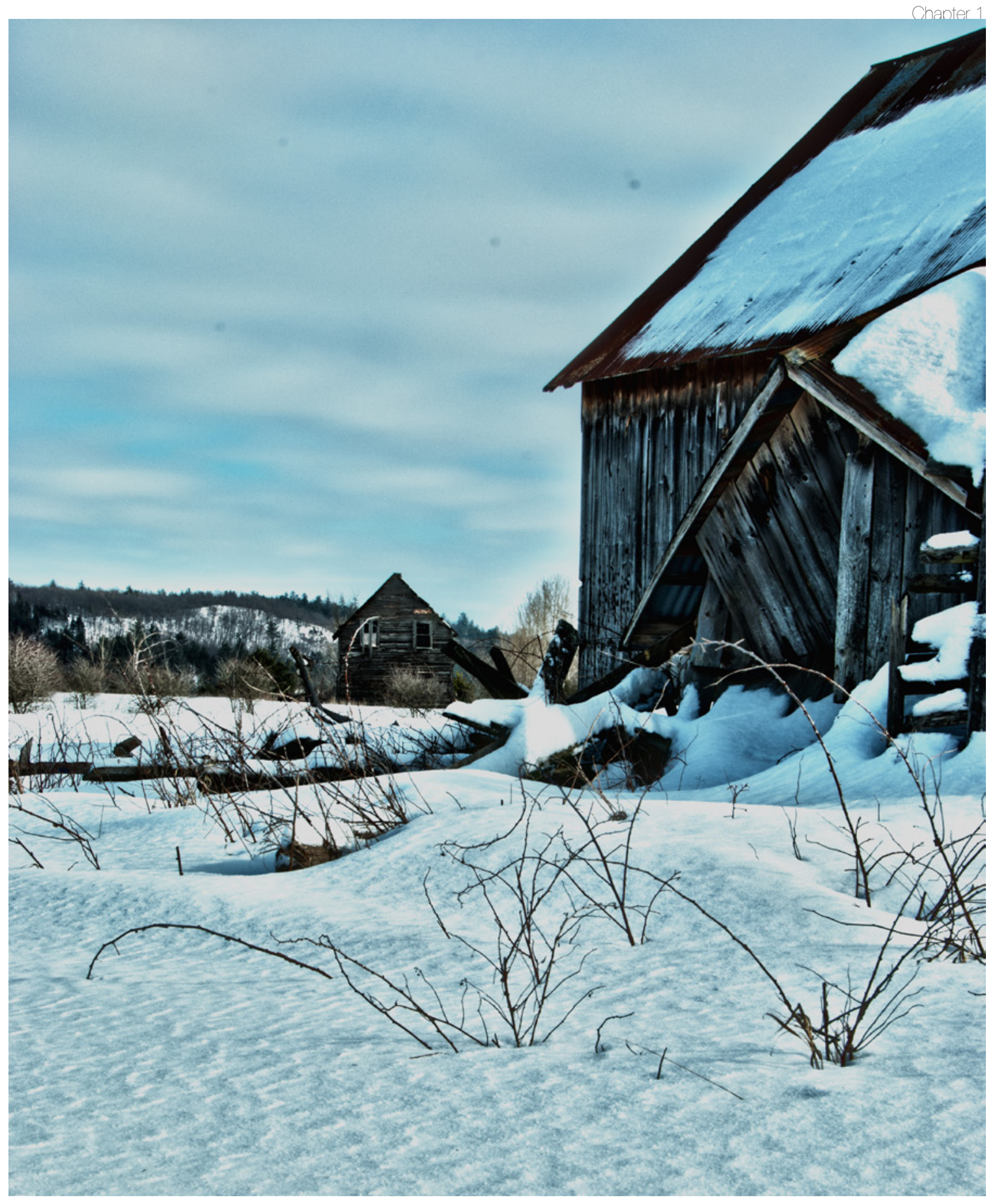

Ottawa River logging-era barn (construction date unknown).

Whitewater Region, Ontario. January 2013. 


\subsection{INTRODUCTION}

Critical Regionalism existed in a realm opposite that of modern Internationalist practice and discourse. The strength of vernacular theory rests on the rigid methods in the way it reacts with the natural phenomena of its direct environment. Through its existence as a traditional building method, it has played on the basic human attraction to historical influences. In the case of the vernacular, these influences have existed primarily as a means of pragmatic decisions that address specific problems, with techniques passed down through first-hand teaching. When Bernard Rudofsky presented his MOMA exhibition 'Architecture Without Architects', it showed a "vernacular architecture where the local population had found ways of using the local materials in response to the local climate that would create radically different vocabularies that were not aesthetic or academic; they were empirical." ${ }^{\prime}$ As an architectural response, the byproduct is a rich architectural methodology specific to a region.

The reactive method of this technique could be constituted as a systematic approach in its application as a middle ground between internal and external forces, which in tandem define the spatial makeup in the development of our built environments. The system of performance becomes a system of localized identity, and the usefulness of the product itself becomes self-evident in the beauty of the work. When architectural design starts to apply

$1 \quad$ Bjarke Ingels, "WorldCraft" (Harvard GSD Lecture, Boston, MA, 4 September 2014). 


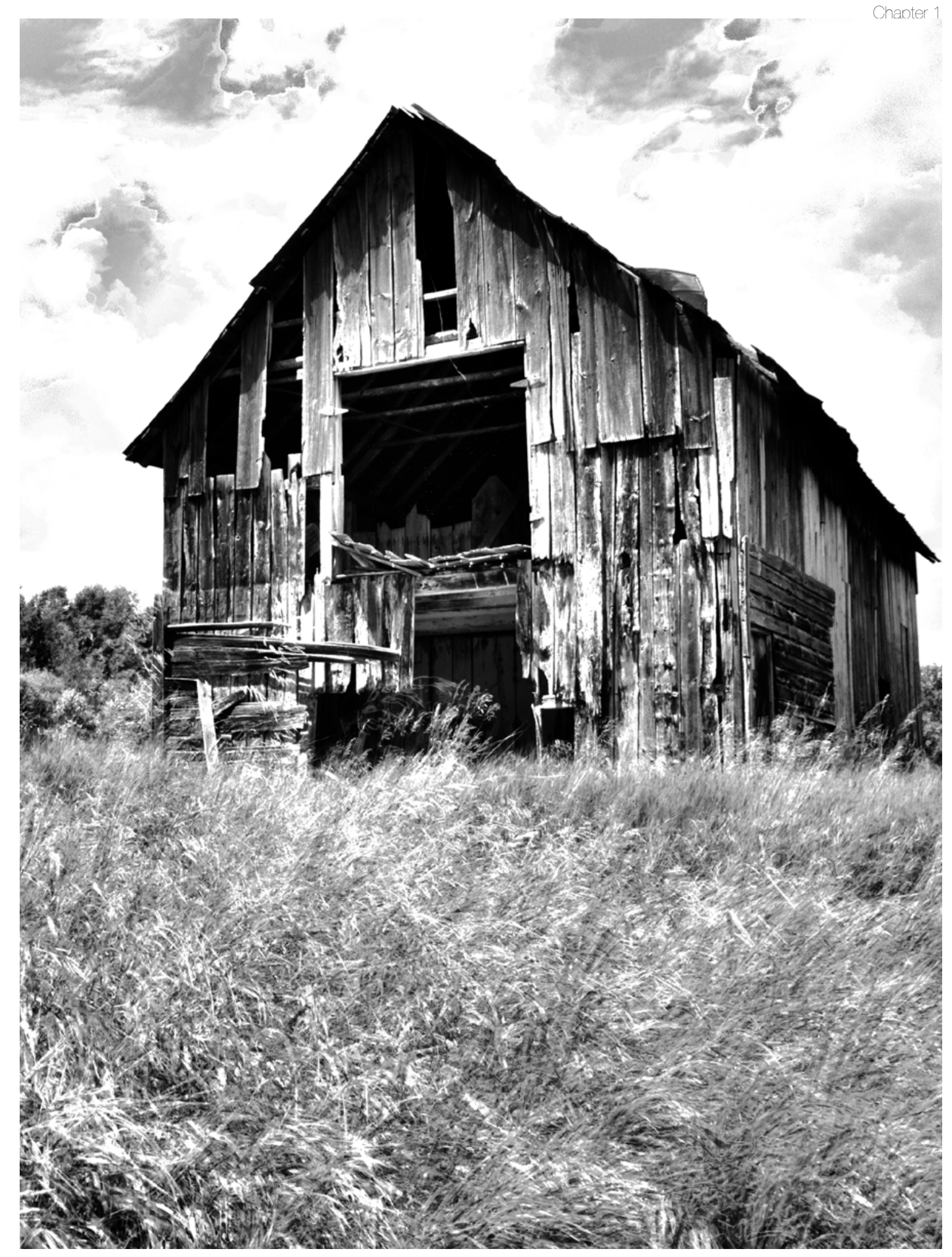

Ottawa River logging-era barn (construction date unknown).

Whitewater Region, Ontario. July 2012. 
vernacular motifs to achieve a solely aesthetic end based

on a set of stylistic rules, it subverts the meaning and circumvents the point. The raison d'etre in the usefulness no longer exists, and the project inevitably becomes a hollow shell of itself.

In a similar manner, the established and evolving strategies of performance-based contemporary design might constitute a new "digital vernacular." In the same way that the vernacular responded systematically to achieve practical ends in design (which itself became the aesthetic image), the digital revolution has allowed a related working method with more precise controls on establishing design criteria. Yet to call it vernacular is misleading, as this suggests dated building methods and materials. To this point it has much more in common with the critical regionalists, which can be applied to localized conditions in various regions independently, without defaulting to the sentimentality of historical kitsch.

The problem with this digital methodology has been the relative ease with which it has been hijacked to appropriate an architecture of image. The appearance of complexity is easier to achieve - and easier to understand - than true complexity in digital project development. Similar to critical regionalism, there are specific contextual times when it should be used; there is no such thing as cart blanche.

The missed potential of digital design tools has not only been lost to an uneducated application. The politicization of whether or not to use digital tools has led to seemingly more effort being put forth to argue each side 
Current relationship across the physical/digital architectural spectrum.

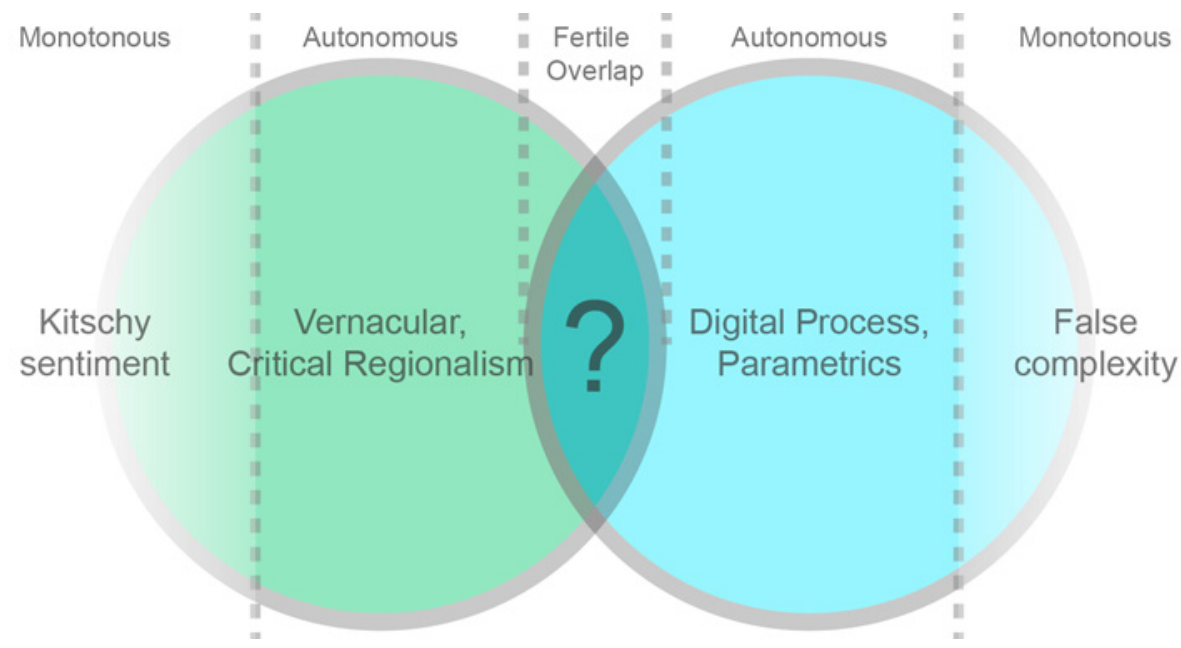

Near-future relationship across the physical/digital architectural spectrum.

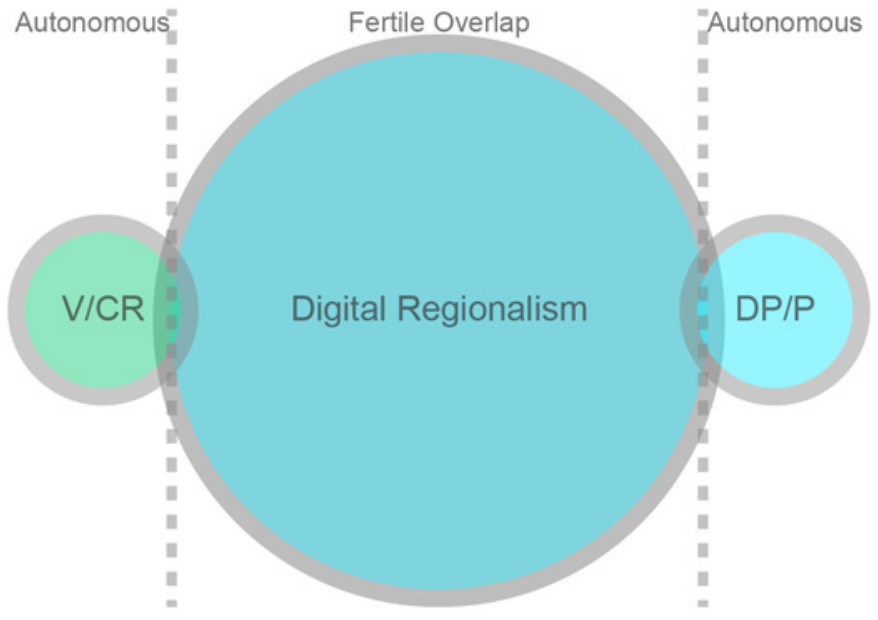

Thesis placement within larger discourse.

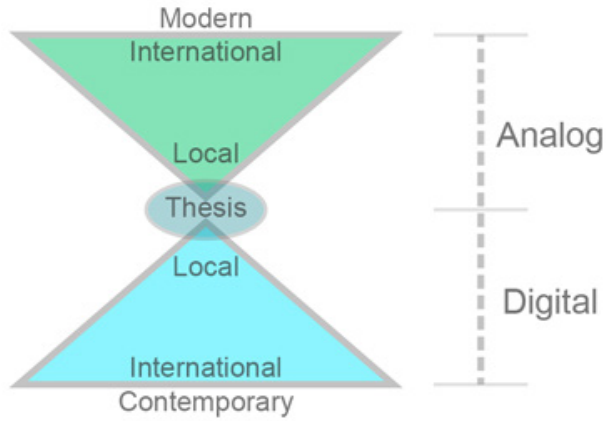


than the effort being put forth to actually advance those

fields of research.

This thesis rejects pondering this question, because it only serves to advance personal rather than collective interest. It is reductive to think that either should exist autonomously in the 21st century, especially when both are searching for a common ground of place-form in defining beauty through functional requirements. Form does not follow function - form is function. The fertile ground of overlap between craft and computer are the focus of this thesis. In the 21st century of architectural design, it is time to shift from a methodology of critical regionalism to one of Digital Regionalism.

In proposing this idea, it raises the question: How can architecture reconcile regional identity drivers with the increasing use of globalized digital design tools for complex projects? The Ottawa Train Station will serve as a case study to develop an architectural proposal in the process of answering this question.

This thesis will:

- Analyze how principles of vernacular craft can be synthesized with the contemporary discourse of digital tools.

- Show how 'place' can be appropriated from the NorbergSchulz era to contemporary ideas of the term.

- Explore a knowledgeable, truthful application of digital tools to provide rigorous analysis in the application of architectural design.

- Seek how contemporary architecture can avoid the pitfalls of utopian ideologues. 
Most importantly, this thesis will strive to show that

there can be a rich overlap between the digital process and

physical craft if the designer chooses to ignore the politics

of their respective hard-liner sides

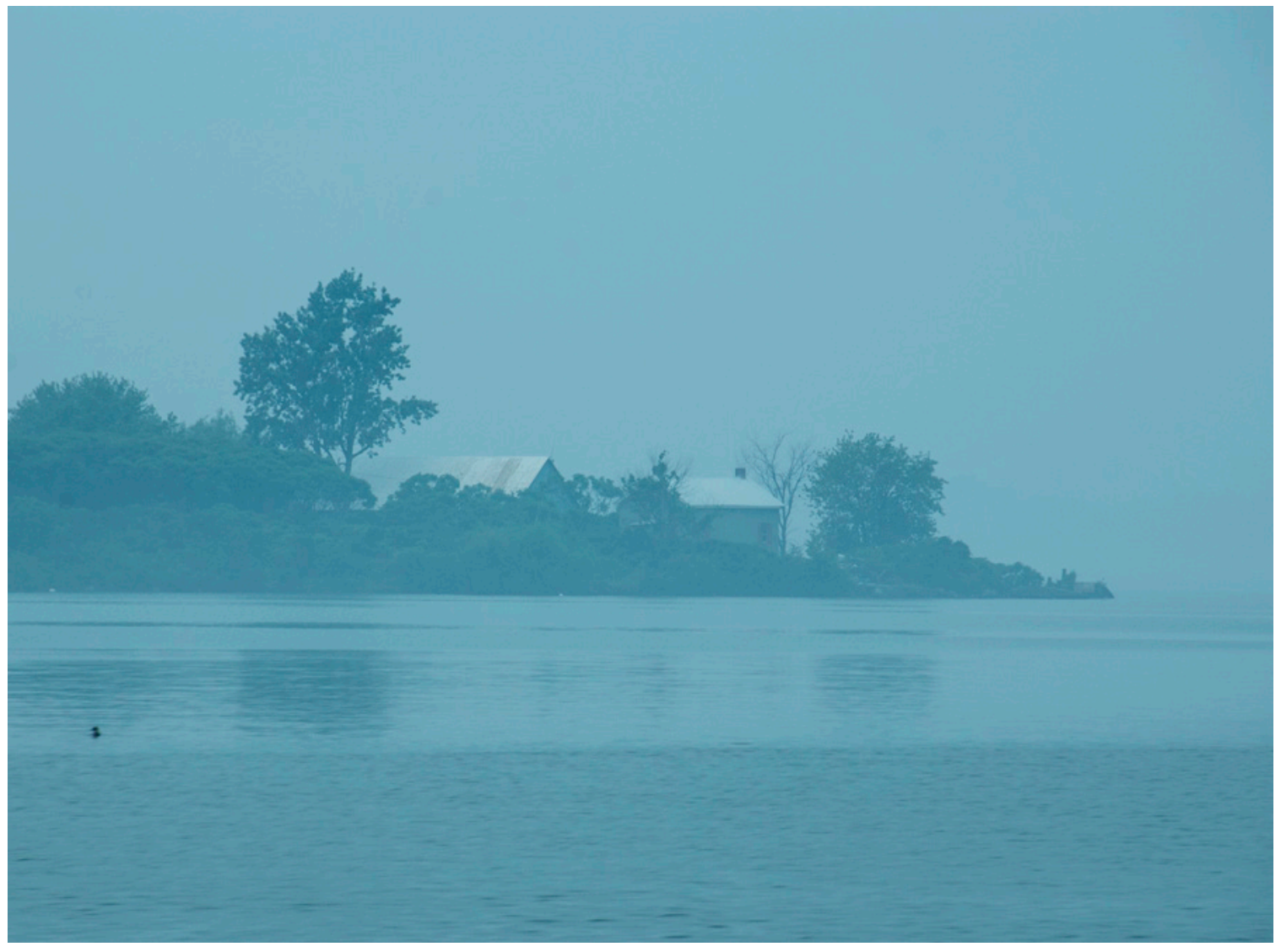

St. Lawrence River barn and house in the elements.

Wolfe Island, Ontario. August 2013. 


\subsection{PROLOGUE}

Key theoretical terms, definitions, and assumptions:

\section{Genius Loci}

Latin description for 'spirit of a place.' The idea has existed for centuries in various aspects with a morphing definition. In this thesis the term is inferred as the contextual characteristics that create the heightened sense of place for inhabitants relating to the specific site and its architecture. This thesis biases Christian Norberg-Schulz's architectural explanations on the subject (particularly from Genius Loci) because of the connection between identity, regional symbolism and distinctive atmospheres associated with the term. The examples and brief case studies touched on as part of the thesis bias Canadian and Northern European architecture. These focused on Snohetta, Sverre Fehn, and Brian MacKay-Lyons for their direct links to NorbergSchulz's ideas on genius loci, as well as Populous for techniques in placemaking for large-scale movement. They have each taken separate positions to the terms and applied them in different ways to their architectural practices. Within the Norgberg-Schulz worldview, this definition assumes a moral imperative for architecture to embody meaningful qualities of the context in which it exists. This thesis assumes that the Norberg-Schulz worldview does not unilaterally apply to vernacular architecture, but can be interpolated and updated to a digital toolset, not only an analog one. 
Vernacular

Describes primary methods of construction, colloquially referring to traditional, folk, or plain historical building techniques. In Ottawa, this is reflected in the deteriorating timber barn constructions throughout the Ottawa Valley and surrounding area. As a basis for this thesis, it appropriates the importance of the long-standing relationship to the economic tradition and historical industry of logging and the Ottawa River as transportation method that developed a material culture in the region. The implication for this is a recognition of regional independence intrinsically tied to the area and those inhabitants living there.

\section{Critical Regionalism}

Proposed by Kenneth Frampton to describe regional architecture practices and schools of thought that "reflect and serve the limited constituencies in which they are grounded."' This thesis biases this interpretation of regionalism for its explicit evolution of the vernacular tradition of functional building, reinterpreted to take Modern and Post-Modern theories into account while simultaneously avoiding kitsch and sentiment. This definition biases Frampton because it subverts surface styles (appliqués) in favor of creating place in the Modern world.

2 Kenneth Frampton, Modern Architecture: A Critical History (United Kingdom: Thames \& Hudson, 2007), 314. 
Internationalism

The overall theoretical association with the International Style for architecture. For the purpose of this thesis, Internationalism is understood as an architecture that places a primacy on a singular, uniform building style that transcends borders, first proposed by Hitchcock and Johnson in $1932 .^{3}$ This interpretation presumes that by doing so, it dissolves notions of region and individualism in favor of globalized design culture.

\section{Functionalism}

For the purpose of this thesis, it is interpreted as a utilitarian architecture that debases human condition from the design, instead producing "new forms [that] would be dictated by sociology and technique alone."4 This definition assumes the consequence of this building method is a loss of place in architecture through an overtly anti-human architecture, biasing ideas of a machine aesthetic instead.

\section{Modernism}

In this thesis, Modernism is recognized as the underlying attitudes toward architecture, as well as art, design, and related subjects emerging in the late 20th century (and should not be confused with the Modernist philosophy of the 17th century, which is not part of this study). Modernism is understood to suggest a new self-awareness to these allied arts that does not automatically follow

3 William J. Curtis, Modern Architecture Since 1900 (New York: Phaidon, 2010), Curtis, Modern Architecture, 209. 
established ideas preceding it.

\section{Cultural Landscape}

Defined by the United Nations as "cultural properties [that] represent the combined works of nature and of man... They are illustrative of the evolution of human society and settlement over time, under the influence of the physical constraints and/or opportunities presented by their natural environment and of successive social, economic and cultural forces, both external and internal." 5 This project concerns the genius loci of Ottawa as a small part of the region's larger cultural landscape, having been shaped through the economy of a material culture. Cultural landscape is extended to include how humans and landscape influence one another through their ongoing morphology, implying that meaningful place is correlated to the overlap of building and landscape. This definition assumes integration between landscape and habitation, rather than an opposition between building and nature.

\section{Identity}

The conditions that produce a cerebral connection for inhabitants to a specific region, where they can associate their architecture to their selves as a collective people. This definition assumes a primacy for human condition and the importance of belonging.

\footnotetext{
$5 \quad$ UNESCO, Operational Guidelines for the Implementation of the World Heritage Convention (July 2012): 24. http://whc.unesco.org/archive/opguide12-en.pdf. 
Meaning

The conceptual purpose that is subsequently projected

from mindful association between inhabitant and

architecture, producing an emotion of affection and importance towards the object.

"Meaning" and "Identity" in this context are intertwined at the most basic level, validating and reinforcing one another symbiotically. A cultural identity for a people is acknowledged because it holds a position of importance in the minds of the collective. It associates the individual to the group. When these associations are collectivized, they develop a communal ownership structure for the group and individual simultaneously. It symbolizes their existence and becomes a totem of significance to the group, creating meaning for their identity.

\subsection{POST-MEDIUM \& DIGITAL METHOD}

In a 2012 interview with Kivi Sotamaa in Helsinki, Peter Testa and Devyn Weiser of the LA-based studio TESTA/WEISER and Sci-Arc design faculty described how the nature of their work is evolving as "the digital paradigm is shifting from the flat Euclidean space of computer modeling tools to a post-medium condition"6. Their outlook presents a poignant measure of the current state of

6 Aalto Digital Design Laboratory, “ADD Discourse." ADD Summary (2013): 25 
an architecture in the throws of foundational shifts. Their

sentiment can be projected to a variety of issues arising

in the profession. "Post-materiality" describes the current

situation where past physical limitations in material science are eroding. The underlying structures are becoming less restrictive in nature and opening new ways of working material into projects. The term is now an apt description for the way architects are interacting with the most elemental, historical materials as they become consistently more digital.

It is setting up a methodology where "the convergence of computation, computational materials, and synchronous robotics breaks down outmoded distinctions between digital and analog or virtual and real,"7 key divides in the profession - and ones with detrimental effects. Defined in the most extreme terms this separation has rendered a politicized debate, with practitioners and academics heavily defending and promoting their respective camps. These two camps have been set opposite each other, but the black and white are irrelevant. The analog and the digital are different systems. One is outdated, so the other is naturally the descendant. It has nothing to do with one being better than the other, since the vast majority of work takes place in the beautiful gray area between them. That is what matters. The question instead becomes how architects retain quality in the built environment - the product.

This thesis arose out of a wariness surrounding 
a widespread, sometimes unquestioning acceptance of digital software advancements in architectural design. In this industry it has not only evolved as a tool, it has revolutionized the way that firms conduct work and business over a relatively short period of time. The onset of the computer in the world of architectural design has contributed to an incredible expansion of possibilities now afforded to architects and designers at a global scale. But what exactly is the relationship of the digital in relation to the physical in contemporary architectural discourse? "Post-material" can be interpreted a number of ways - the most obvious as a method for ignoring the realities of construction. Yet Testa/Weiser approach it more subtly, taking an attitude where "we test ideas against materials and materials against ideas in response to the unexpected qualities and virtual ties of materials." ${ }^{8}$ In questioning what the material intricacies can reveal to the designer, they are able to synthesize a new methodology for working methods that leverage physical traits through digital processes.

\subsection{HIDDEN TRUTH AND FALSE} COMPLEXITY

It is now common in contemporary practice to see a divide slowly establishing itself in architectural processes in terms of the pre-digital and post-digital. For much of the 
students now graduating from post-secondary institutions,

there does not exist a time in their worldview of projects

done entirely by hand.

Whether it be due to practicality or staunch

distrust, it is not uncommon for firm principles trained

during that time to reject the computer in their own work

today, and for many university professors to promote

similar sentiments - although this seems unlikely to be the

case for their younger counterparts. As the computer only

came into widespread architectural office use towards the end of the 20th century, there are still a large number of architects working who trained completely through hand drawing and manual modeling. Why has this happened? "The common objection to computer-generated design is the perceived handing over of responsibility from a human to a digital agent. And yet, the generation of form according to some criteria other than individual taste is, and has been the objective of many, whether a set of 'Godgiven' formal rules (Classicism), a set of structural and/ or programmatic requirements (functionalism), or a set of environmental parameters (bioclimactic architecture)."9 Therein lies the fallacy of the digital/physical divide: one should not reign supreme over the other. Hierarchies in design discourse have always been present in academia; only the guidelines have changed. Rather, this suggests that the autonomous working method of either/or is inferior to the integration of the two as both/and. There also exists a level of narcissism to the autonomous argument: to claim

9 Susanna Hagan, Digitalia: Architecture and the Digital, the Environmental and the Avant-Garde (United Kingdom: Routledge, 2008), 78. 


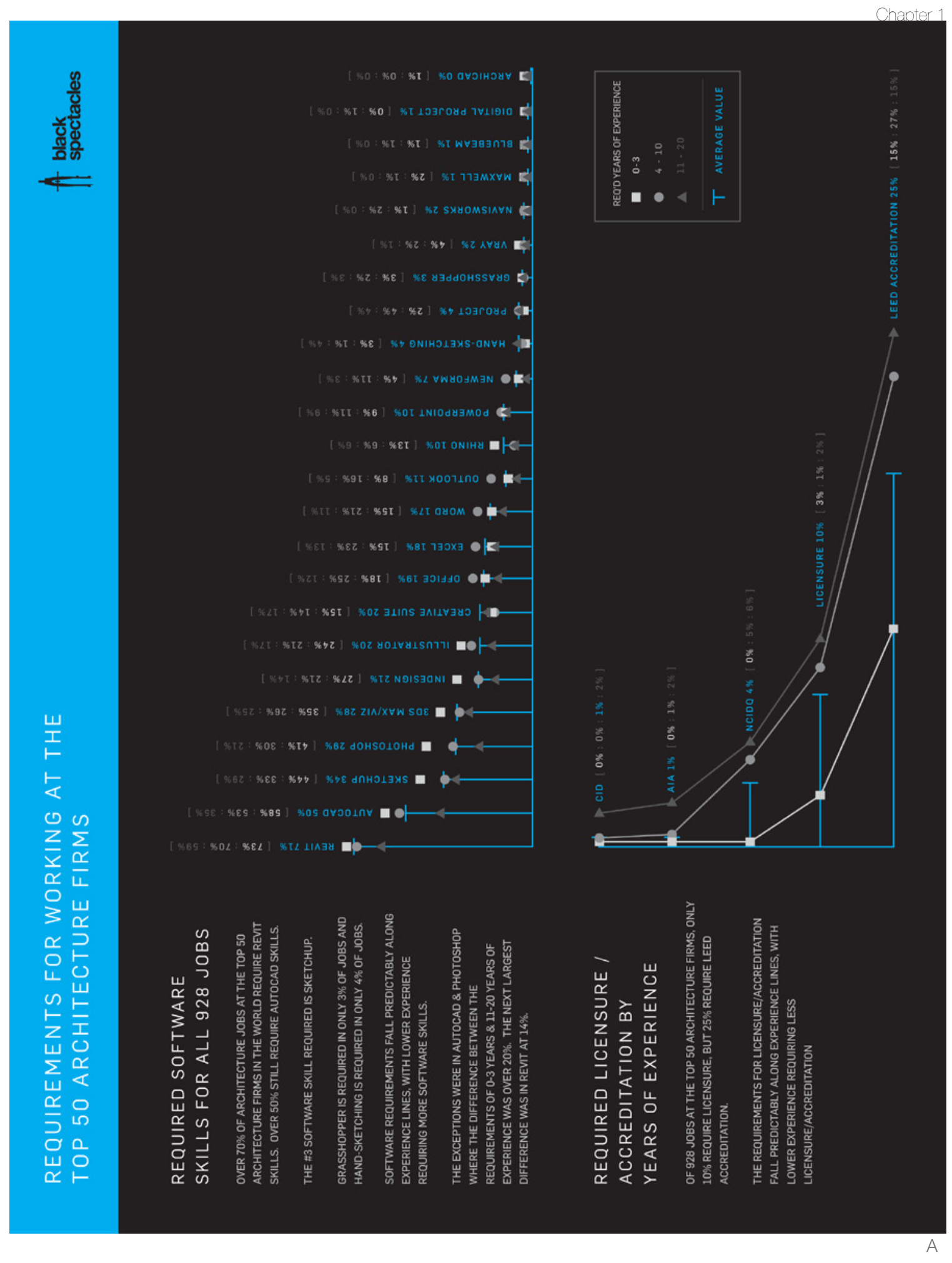


one doesn't need to acquire any useful software knowledge

is also a subtle claim of being above it, or as David Rutten

describes, the motivation for a wider "institutionalized

yearning for recognition." 10

Meanwhile, a critical mass has developed for architectural software familiarity. The skills consultancy firm Black Spectacles assessed nearly 1000 job openings from Architectural Record's July 2013 survey of 300 high-profile architecture firms across the world, and compiled the results into a graph. ${ }^{11}$ A quick look at the key industry-standard software packages shows a descending importance for Revit (71\%), AutoCAD (50\%), SketchUp (34\%), 3DSmax (28\%), Adobe Creative Suite (20\%) and Rhino (10\%). Comparatively, hand-sketching is near the bottom (4\%) in terms of desire, while hand-drafting was not even included in the questionnaire. This is not to dismiss hand-drawing itself as irrelevant; it simply points to shifting hierarchies in desired skills. With the recession of 2008 affecting the architecture/construction industry as well as young people disproportionately, it makes no sense that recent graduates should remain tied to analog working methods for the sake of a perceived purity.

Rather, the expansion of digital discourse in the academic world has lead to a new default in working methods. The evolution of digital tools provides opportunity for new levels of complexity because "the

10 David Rutten, "Worrisome Trends in Architecture Education," I Eat Bugs for Breakfast Blog, 9 August 2013, https://ieatbugsforbreakfast.wordpress.com/ 2013/08/09/ worrisome-trends-in-architecture-education/.

11 Marc Teer, "Requirements to Work in the Top 50 Architecture Firms," Black Spectacles, 27 June 2013, http://blackspectacles.com/blog/software-licensurerequirements-to-work-top-50-architecture-firms\#.VK1ZhSf31o4. 
computer enables the architect first to represent that

complexity... by taking over the calculation work - the computing." 12 Whether this promotes the use of non-linear form is hard to say; it simply makes it easier to work with and creates a viable fabrication process should that be the best working method. The problem arises when the default begins to inform the question before the answer, with Christopher Alexander pointing out that it could push the designer to distort the view of the question so that it aligns with a specific tool, exposing a superficial relationship ${ }^{13}-$ when one has a hammer, every problem looks like a nail. He goes on to describe that one should avoid a default of trying to apply a computer to a design problem, and instead wait for a complexity to be overwhelming so as to warrant a digital resolution. ${ }^{14}$

What is also becoming easier is the use of software programs to develop complexity in architectural design ad infinitum. When used with skill this can begin to unveil inferred hidden realities and solutions of a design problem. But, as Rutten ${ }^{15}$ points out, "it's easier and easier to call high-level functions and make a geometrically involved algorithm in just a few minutes. This is typically known as 'progress,' but there's a dark side" to the indiscriminate use of digital systems and tools, whether they are algorithmic, parametric, generative, or some other offshoot. As the inventor of the Grasshopper platform, he worries that "computation can be used to generate large amounts of

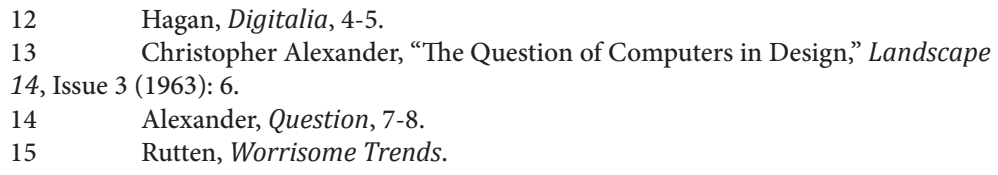


complexity, but complexity does not equal worth." ${ }^{16}$ When

the digital dilettante chooses to use software as a means

of achieving complexity to obscure and obfuscate, the

result can only be associated with a search for newness and

pure form. "They are tools, they are not content. There's a

difference, and if you don't have anything to say with your

tools, there's no point in using tools."17 These are shallow

goals for a meaningful architecture, existing without critical

application. In these instances it is a bastardized offshoot

"where complexity masquerades as information."18

The architecture that arises from these self-

referential pursuits was not the point of a digital revolution;

it has so many more possibilities. "Architecture as 'pure'

form - that is form that is not answerable to any criterion

beyond sensory pleasure - cuts relatively little ice" ${ }^{19}$ and

lead to the rejection of overly expressive design because

they exist as "signifiers of nothing in particular beyond the

significance of signification." ${ }^{20}$ The self-referential aspect,

where architecture exists in a vacuum, is therefore an

unworthy goal for the digital revolution.

Schumacher's autopoiesis exceeded the need for a contextual methodology - an autonomous Modernism par excellence. But just like the utopian idealists of Modernism, Schumacher's architecture-as-object argument disregards the realities of context. The system should not be autonomous, it should reinforce the other contextual

\footnotetext{
16 Ibid.

17 Elizabeth Timme, interview by Paul Petrunia et al, Archinect Sessions Podcast 11, 22 Jan. 2015.

18 Rutten, Worrisome Trends.

19 Michael Sorkin, “Critical Measure: Why Criticism Matters," Architectural Review 1408 (2014): 92.

20 Ibid.
} 
systems around it that invariably needs to interact with in tandem. Once the architecture becomes contextualized it becomes subject rather than object. If the designer forces the users to choose one autonomous system or the other, it devolves the work to segregated design and handcuffs future integration. To ignore this limits the qualities of the computer to compute relevant information from the site and contextualize the digital architecture into the integrated system. This expunges Schumacher's claim that the digital exceeds the need for a contextual methodology.

This context can provide the referential aspects that Sorkin belies, and as a focus back on true things can attain (or at least move towards) Rutten's assertion that "the only time is makes sense to invoke computation in the design process is when there is some relevant data that needs to be computed." ${ }^{21}$ It should be noted that while Rutten specifically uses the word 'data' here, this acts as a signifier for tangible things (ie. movement, growth, sunlight, expansion, direction, distance, etc.) and not simply Excel charts. Associating a digital approach with a contextual approach to the tangible site characteristics to generate an architectural response acts as a safeguard to many of the problems conjured: "insisting on autonomy is both indispensable and complete bad-faith. The link to agency makes all the difference." ${ }^{22}$ To have agency means to have an objective in mind; architects must reject the pursuit of a false complexity, which will inevitably produce faceless architecture, and instead turn to a digital method that 
promotes the use of new tools as means to an end when

human potential alone has been exhausted.

A) Black Spectacles, "Software Requirements for Working at the Top 50

Architecture Firms. http://blackspectacles.com/blog/software-licensure-

requirements-to-work-top-50-architecture-firms\#.VK9GOid8Mmc 

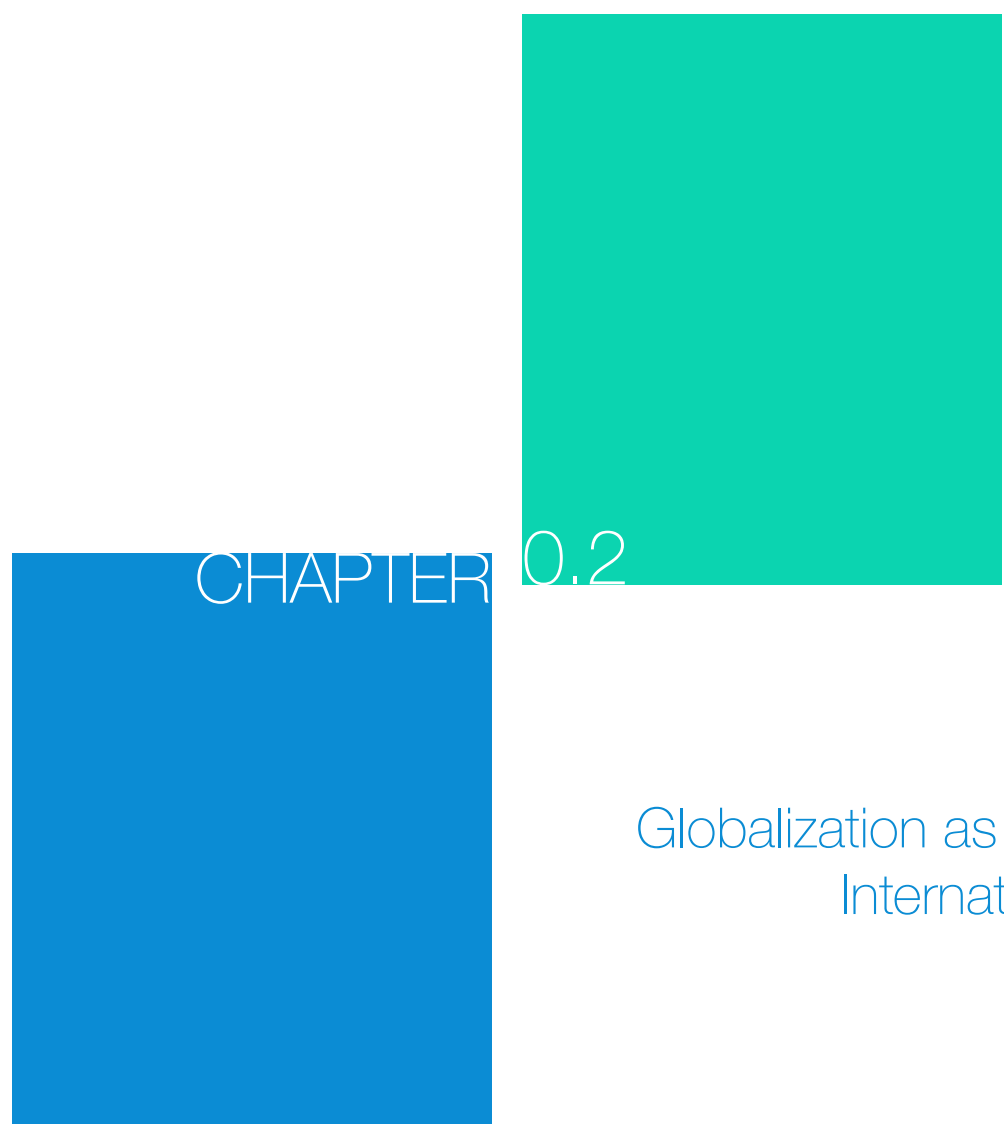

Globalization as the Re-emerging Internationalism 
_ Juhani Pallasmaa: "If I think of no identity, of architecture without any identity, would this be globalized architecture?" _Peter Zumthor: "Probably." 


\section{$\underline{2.1}$ FORM / FUNCTION}

Globalization and the inherent transnationalism

of the contemporary world have ensured a profound

connectedness. This alone is not necessarily negative;

the ability to transcend physical boarders is a testament

to the social-geopolitical understandings reflected

in contemporary society. Any reader most likely has

a smartphone in their pocket or within a few feet of

their relative position. With this device and a good

service signal, that individual can open Google and

instantaneously find a slew of search results to any subject

of their whim - the proverbial world at your fingertips. This

aspect of globalization has, admittedly, offered a revolution

in cultural understanding through an accessibility to

information overload for anyone who seeks it.

However, globalization has managed to transcend boarders, to the point where it has blurred the lines of what distinguishes regional identities from others, going far enough to have "the tendency to wipe out national and cultural differences"1 from one area to the next. This may be largely due to the fact that people can, through this newfound connectivity, begin to blur the reality of their own culture with those elsewhere. This is the key downfall with the resultant homogeneity, as "globalization is typically cited as the antithesis of cultural anchoring. In this context, it is seen as a threat to cultural diversity and linked to a fear of uniformity and a loss of cultural

$1 \quad$ Kjeld Kjeldsen, forward to New Nordic: Architecture \& Identity (Denmark: Rosendahls, 2012), 10. 

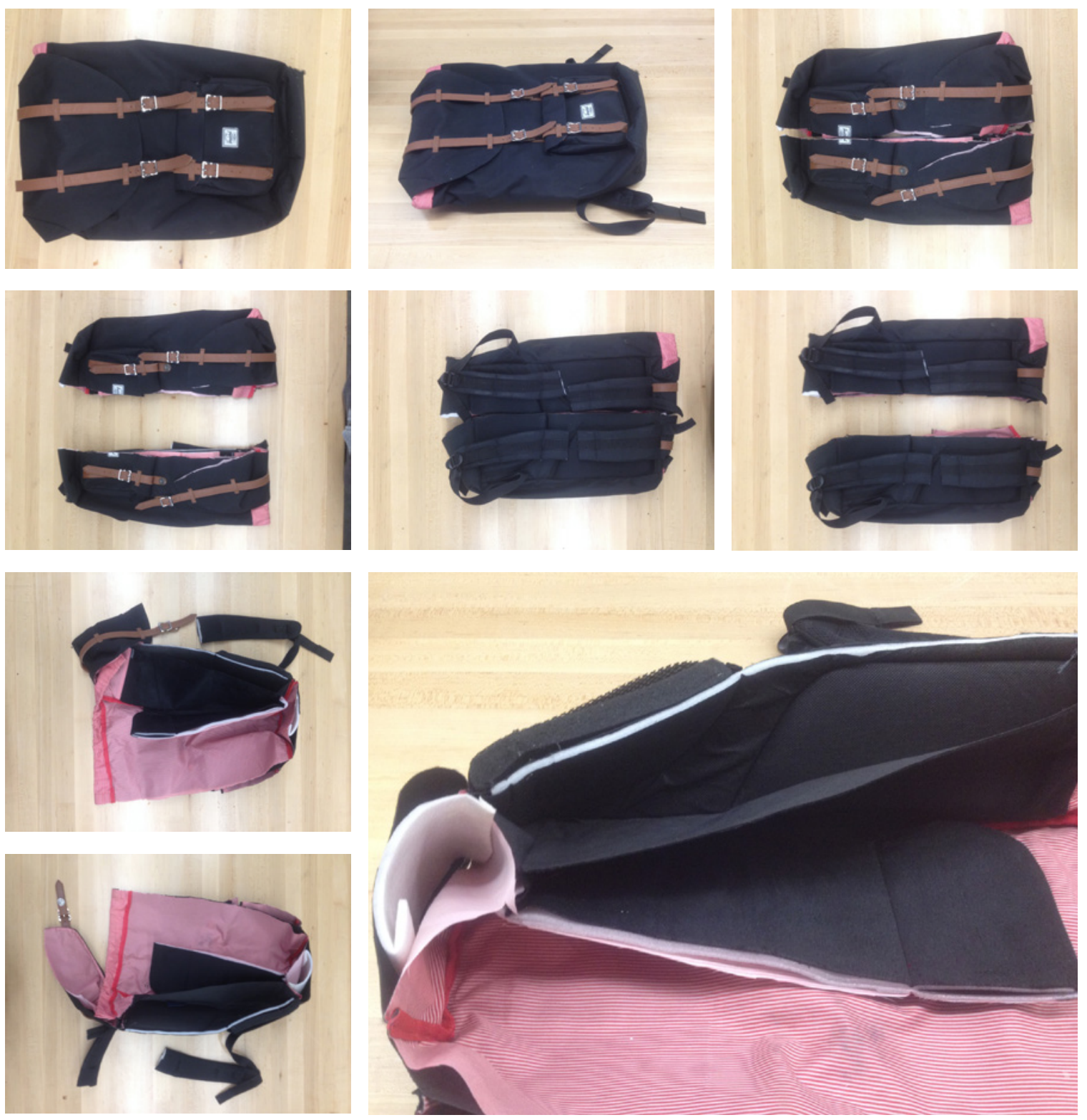

Dissected Herschel backpack revealing globalized material palette. 
distinctiveness." ${ }^{2}$ The effect is similar to that established

during the Industrial Revolution, as the institutionally

embedded pragmatism of the working machine replaced

vernacular handicraft. And it is not unreasonable to be wary of the effect of globalization on an increasingly

digital population. Uniformity can lead to destructive homogeneity if it is centered on overly functionalist ideals.

"Contemporary design computation is more aligned with Modernist thinking where the machine analogy dictated frictionless operations of closed spatial objects... Unplanned human interference was designed out, leading to cognitive dissonance and, ultimately, dysfunctional use"3 - the way a society thrives as a cultural identity is directly related to their built environments, and vice versa. This homogeneity has manifested itself in the ease of creating non-rational geometry and is particularly prevalent in examples such as the onset of the spline, which can "[mathematically] join a set of nonaligned geometrical points with a continuous curve."

While the feasibility of complex projects has become exponentially more manageable, the ability for limitless design via the computer has lead to a globalization of design sensibilities, with sleek surfaces becoming ubiquitous with the cutting edge. The trend, when viewed in a wider perspective, draws similarities as a contemporary link to ideas fronted by the Internationalist movement from the 1970s with the "loss of perspective characteristic

$2 \quad$ Michael Anderse and Jeanne Schelde, "Architecture Gives Shape to Our Existence," in New Nordic: Architecture \& Identity, (Denmark: Rosendahls, 2012), 35. 3 Christian Derix. "The Space of People in Computation," Architectural Design 231 (2014): 15.

$4 \quad$ Mario Carpo, "Breaking the Curve," ArtForum International 02 (2014): 169. 
of modernity, the collapse of depth, the premium of the surface." 5 This premium of the surface exposes the current discourse to similar failure if followed dogmatically. As Paul Coates points out, "The functionalist tradition has suffered many blows in the last 50 years, partly because it was always an oversimplification, and partly because technology has now reached a point where the constraints of structure have almost vanished, with form becoming the precursor of function rather than its determinant."6 This represents a shift in the way functionalism has been interpreted from Modernism to the contemporary; form no longer follows function, but rather supports and enhances it. It is the evolved manifestation of Reima Pietilla's decree to move away from the form-follows-function maxim, as he recognized the banality of the towers-in-the-park and the failures it represented. Pietilla was "advocating instead a more open-ended and accommodating proposal 'form follows approach.' By separating the idea from the form, Pietilla successfully invalidated the conventional notion of function as the controlling or dominant force in the field of architectural design." ${ }^{7}$ It's a common fallacy that a postfunctionalism discourse be wrapped in excessive expression - though it certainly is a symptom of its sickness. The freedom to disembark from that rigidity instead needs to focus on the form exercising and reinforcing programmatic requirements - a true function, rather than the function

\footnotetext{
$5 \quad$ Anthony Vidler, Warped Space: Art, Architecture, and Anxiety in Modern Culture (Boston: MIT Press, 2000), 239-240.

6 Paul Coates, "The Deep Structure of the Picturesque," Architectural Design 231 (2014): 35.

$7 \quad$ Malcolm Quantrill, Plain Modern: The Architecture of Brian MacKay-Lyons (New York: Princeton Architectural Press, 2005), 35.
} 
of structure. It is accepted that "the white-plaster walls and planar surfaces so often employed in the 1920s were perhaps intended to convey a non-material quality, to suggest the opposite of handicraft: the abstraction of the machine"8 - but humans are not machines, no matter how closely they integrate their work flows. This played to the alienation of spaces in Functionalist architecture, with the idea of Functionalism as a performative design. The minimal use of endless concrete columns (in relation to the lengthy load bearing wall systems previous) to support a utopian ideal for building culminating in the towersin-the-park model that directly supported this machine aesthetic.

The oddity is that this partially drove the problem of placelessness during Modernism - the failures of the Towers in the Park are well documented. Structural functionalism could feed the aesthetic, but could not produce worthy 'places'. The machine aesthetic of the 1920's periodically morphed and developed into various forms, with the lineage moving along commercial Modernism (1950’s - 1970’s), Post-Modernism (1970’s - 1990’s) and Deconstructivism (1990's - 2000's). By the late 1980's and 1990's the idea was in a state of failure.

In the 2000's, however, the digital revolution has freed structure from functionalist rigidity - and thereby allowed a productive method to create 'place' from that evolution. The emerging techniques of the digital design tool set mean it is able to reject the common weakness

8 William J. R. Curtis, Modern Architecture Since 1900, $3^{\text {rd }}$ Ed. (New York: Phaidon, 2010), 268. 
of sterile banality in this evolution of the Functionalist

machine aesthetic.

It is time to leave that dated theory behind and embrace what Christian Derix calls a new use aesthetic, which "affords an empathic correlation between computational heuristic and spatial experience which will require the synthesis of formal and system aesthetics." In the computational, Derix's 'use aesthetic' creates an architecture that responds to requirements of spatial function, based on prevailing needs. This is distinct from the Functionalist ethos, which simply minimized the architecture into ambiguity; free-flowing space between endless columns defines nothing. Instead, the use aesthetic makes space by defining necessary boundaries. In terms of vernacular, the 'use aesthetic 'correlates the material choice to localized culture as a visual representation. It has its own use aesthetic that resonates more prevalently at the empathic level. In the transition from digital use aesthetic to physical use aesthetic, the "field of immaterial ciphers is substituted for the material traces of the object"10 which give evidence to the existence of phenomena in the object. This is most prominent in connection to time and weathering, as evidence of the resilience in their material culture.

Unfortunately it now seems that the ideal from the minimalist agenda of overt simplification, "that is, simplicity through reduction - in order to promote

\footnotetext{
9 Derix, "Space of People," 22.

10 Stan Allen, Points \& Lines (New York: Princeton Architectural Press, 1999), 15. 
complexity within the whole"11 has swung too far to

the other side. Where this reduction boiled down to a homogeneity of similarities, sleek digital surfaces are now blending together as a homogeneity of newness. The literal use of symbology shows that "these images mark a shift from models of formal organization and meaning that work with transparency and depth to a condition of shallow surfaces, in which meaning resides in graphic information lying on the surface."12

To outright reject the influence of the hand/mind/ body in search of a perceived autonomy only reinforces the inherent problems of sterility in design. Overlap between digital and physical must be achieved because "architecture works simultaneously with abstract images and with material realities, in complex interplay. It is a material practice." ${ }^{13}$

\subsection{PHYSICAL / DIGITAL}

Similar to the Arts \& Crafts movement that happened as a reaction to the Industrial Revolution, now in contemporary design "the tradition of craftsmanship is clearly gaining increasing value and appreciation in today's reality of the technological world" as these reflect the traditions of a people's identity. ${ }^{14}$ Just as the individuals involved around promoting the Arts \& Crafts as an

11 Robert Venturi, Complexity and Contradiction in Architecture, $2^{\text {nd }} E d$. (New York: Baronet Litho Inc., 2007), 18.

$12 \quad$ Allen, Points \& Lines, 49.

13 Allen, Points \& Lines, 51.

14 Juhani Pallasmaa, The Thinking Hand (Italy: John Wiley \& Sons, 2009), 51. 
alternative to the monotony of a machine-aesthetic, so

too does an appreciation for work of the hands offer an

alternative to the surface aesthetic of many contemporary

projects.

It is precisely the truth in the act of physical things interacting with the hands that activate the body, as "hand gestures are, naturally, an important aspect of the arts of rhetoric and acting." ${ }^{15}$ This acting, as an extension of agency, is what needs to remain in the design process when incorporating the digital into the physical. To lose this agency and submit to autonomy is one problem of a non-synthesized, disconnected system, where "automation tends to turn us from actors into observers" of the objects on the screen. ${ }^{16}$ This disconnect creates a false reality, with the architect submitting control to the machine as a default, similar to the Functionalists and their machine aesthetic. As a counter, "the task of the critical, profound and responsible architect is to create and defend the sense of the real." 17

Working through physical creation is a method of establishing the real. Humanity, however, rarely has shaped his objects through handiwork alone. Rather the ingenuity of various tools has steadily increased production from era to era. "Tools evolve gradually through a process of small improvements, use and rejection"18 just as much as the repetitive nature of a skilled craft is learned via
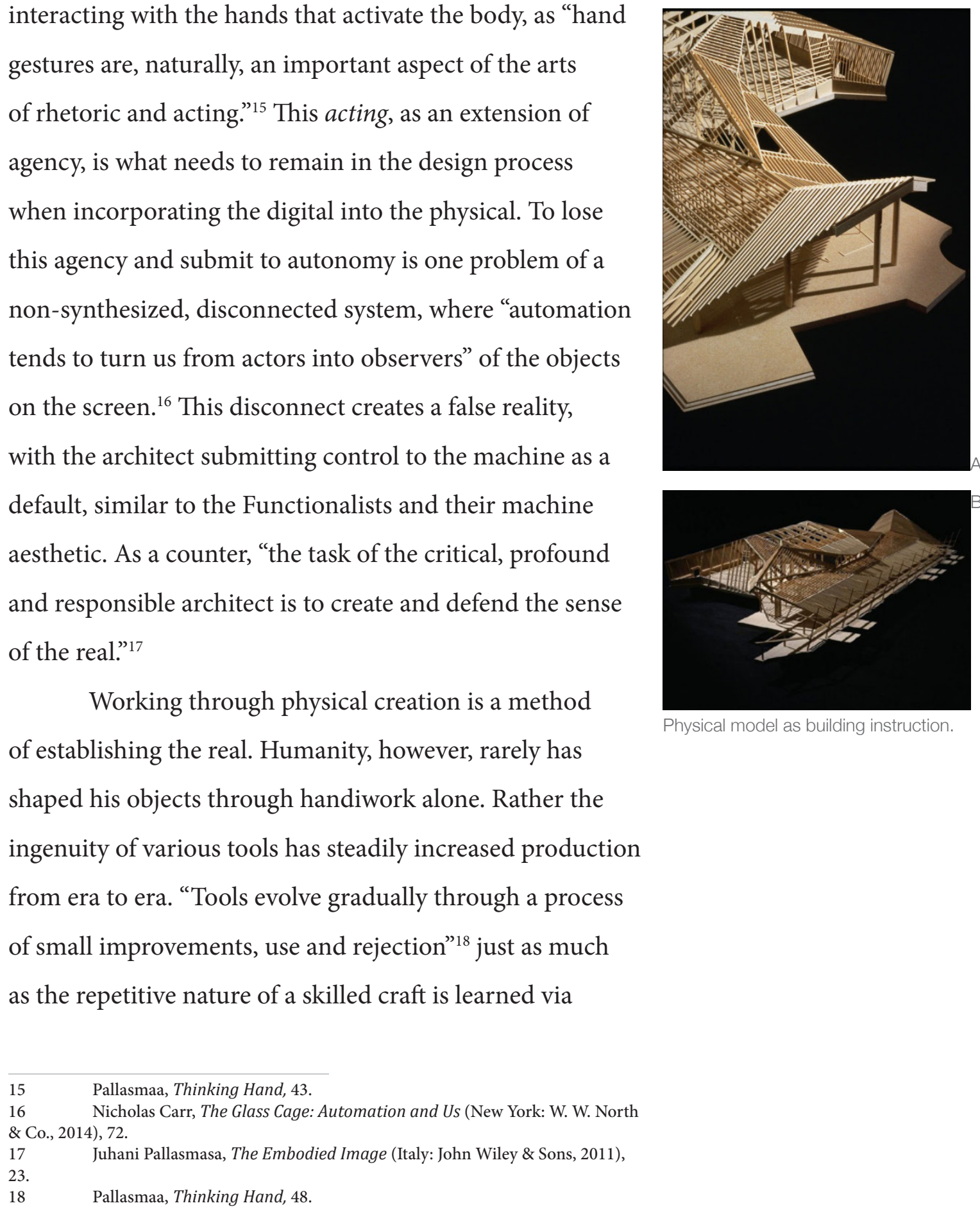

Physical model as building instruction. 
the apprentice's mind correcting its hand through minor

shifts, over and over. The tool is not distinct from the hand when in contact, but rather "the tool is an extension and specialization of the hand that alters the hand's natural powers and capacities... it has transformed into an entirely new species of organs, a tool-hand." 19

However, the traditional tool is based around fixing a physical object, and editing its exact being. The working method of physical tools also has a slightly different translation to those of the digital realm, which constitute broader systems. With a potential risk to the flesh in any craft, a craftsman must be mindful in avoiding physical harm by a slip of the hand. With a direct consequence, the craftsman can learn to work with the flaws of his tools as required. To the contrary, digital tools, particularly scripting interfaces like Grasshopper, are based around a large group of tooling elements that the designer crafts into his own digital tool set according to the specific need. If the tool is no longer working as intended, the actual tool (not the object/project) is changed or updated. The editing of flaws is therefore different at an essential level - but it still constitutes a unified entity between tool and user. It is simply not embodied - the digital craftsman corrects himself through a natural, unconscious intuition, rather than an conscious reaction.

Pallasmaa describes that through repetition as means of improvement, the connected eye/hand/mind can intimately understand the problem so well that he becomes 
accustomed to the process as a responsive reaction to

an action. At this point is where the natural intuition becomes true, and the craftsman can use his built-up instinct to produce. As the maker can at that point trust that their instinct is correct, the embedded wisdom of the physical material interacting with the maker becomes a pure reflection of that identity, since at that point "the work seems to be producing itself." ${ }^{20}$ The refinement of a series of intuitive movements constitutes an autonomous reactive system as a reflection of the embodied wisdom of the connected, singular hand-eye-mind response to the material condition it is engaging with. The wood carver understands how to whittle with the grain rather than across it. The automatic method exposes the physical human condition to disconnect from the digital realm because of its removal of the tool's tactility, the very haptic qualities it embodies.

Pallasmaa gives two main points for architecture to address, as it occupies "a double task: the practical function of life and activities, and psychic function of establishing our mental foothold in the lived reality." ${ }^{21}$ The disconnects described above appear to occupy scenarios where one has overtaken the other; the functionalists created unlivable places, while the digital dilettante has tried to obfuscate the lived reality through false complexities. The computer is an integral part of the contemporary design process. It can help to infer information that would otherwise be unattainable, and in its own way complete a set of 
information to a reasonably accurate degree when that

would otherwise be impossible. However, the computer

does not recreate the inherent physicality that is established

through the process of making. Because of this, it leaves out some inherent material subtleties. This does not by default make it subordinate; rather it is simply a factor that must be acknowledged during the design process. 


\subsection{INVESTIGATION I:}

\section{RECONSTRUCTING PHENOMENA}

The proliferation of a variety of digital design tools has expanded the possibilities of what designers can investigate and produce. In beginning to bridge the gap between the physical object and digital representation, the first set of material analysis dealt with attempting to recreate existing organic conditions found within the Ottawa region. Particularly, how to separate these elements into different parts and understand them simultaneously in isolation and congregation. Understanding elemental structures of regional phenomena can inform translative methods for defining building form via regional associations of identity through existing material culture.

This produced two digitally reconstructed knots for $3 \mathrm{D}$ printing, a surface separation between the hardnesses within the wood, and finally an isolation of the growth rings inside destroyed logs. 

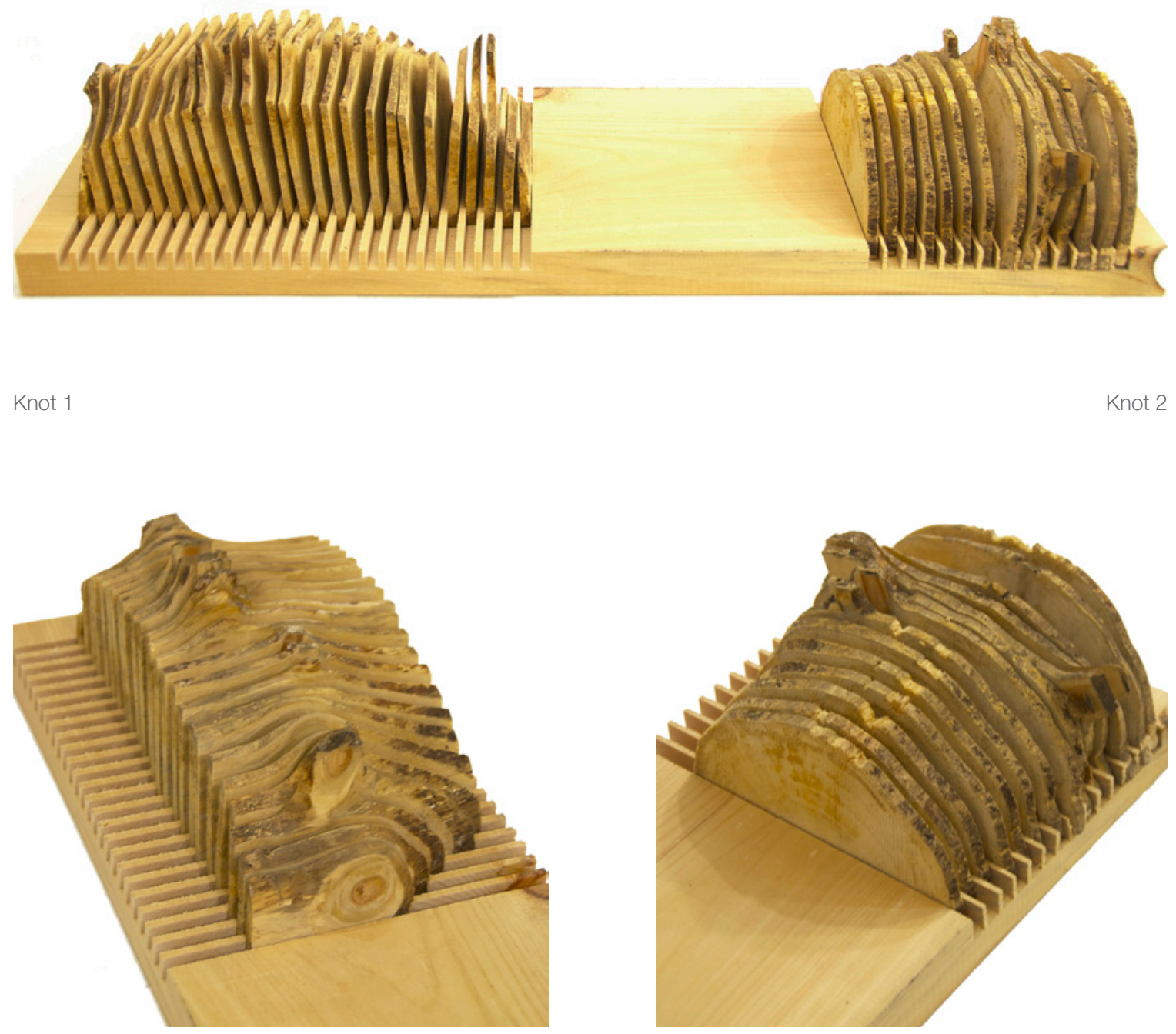

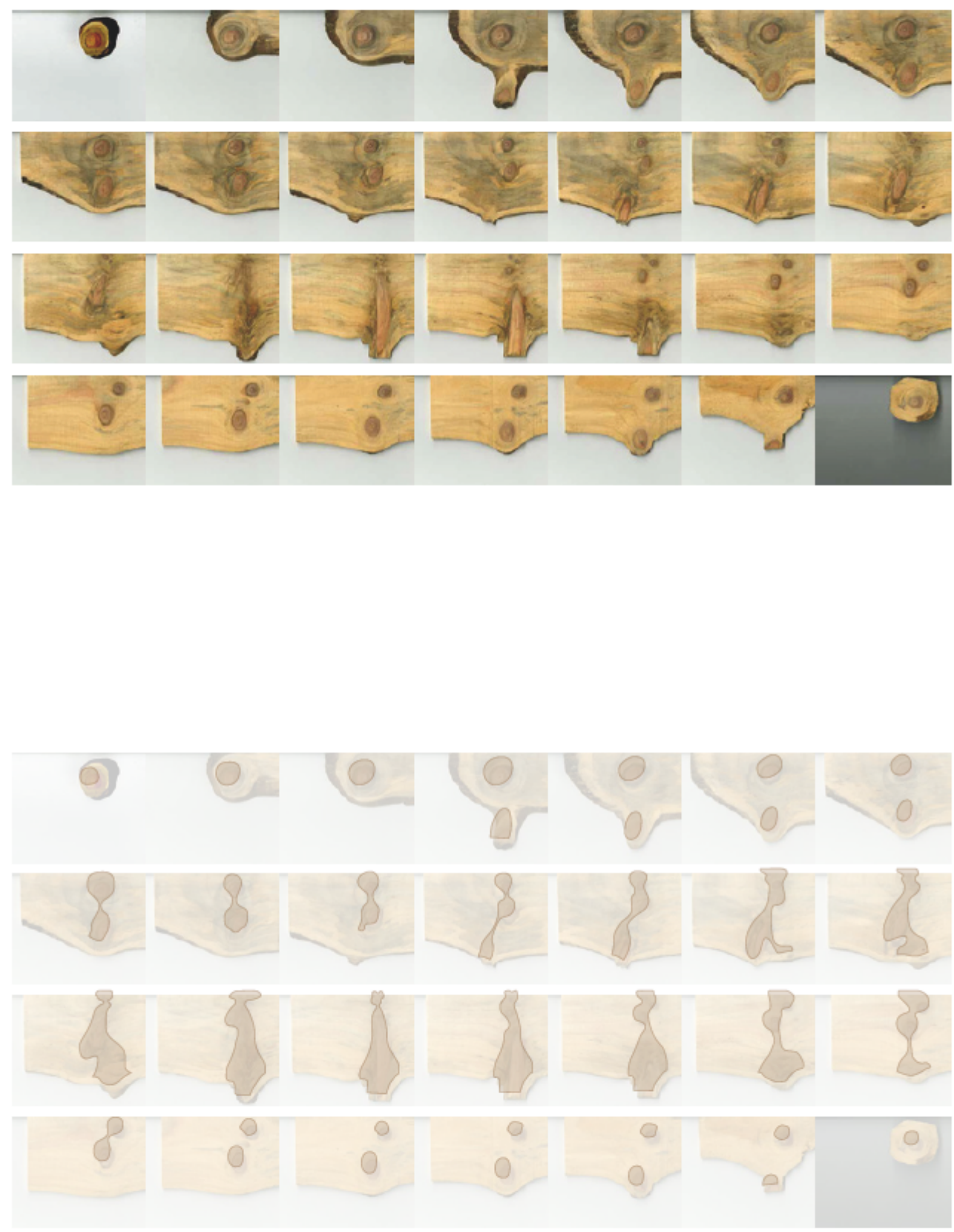

Knot 1 Sections 
Chapter 2
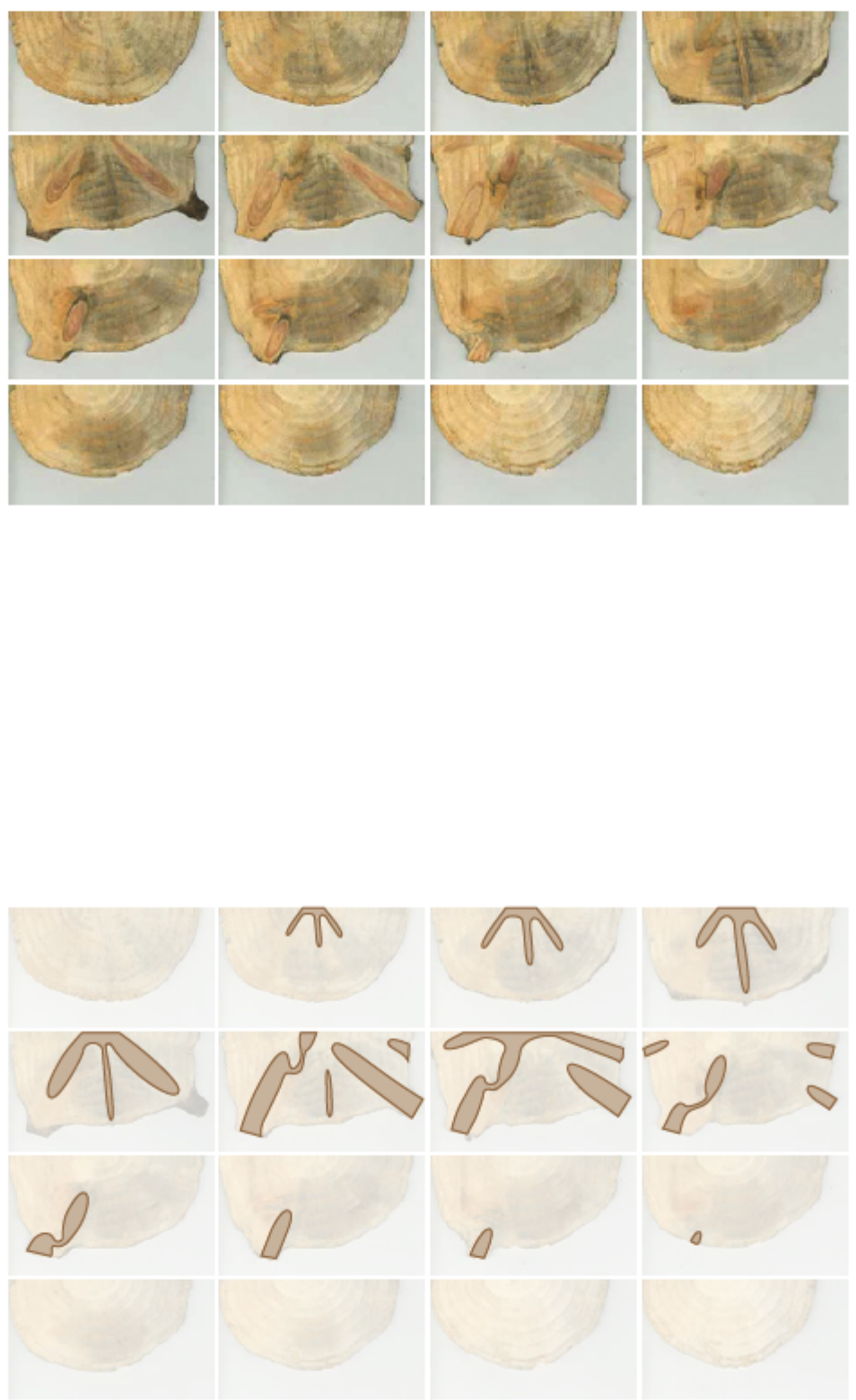

Knot 2 Sections

$$
-41-
$$



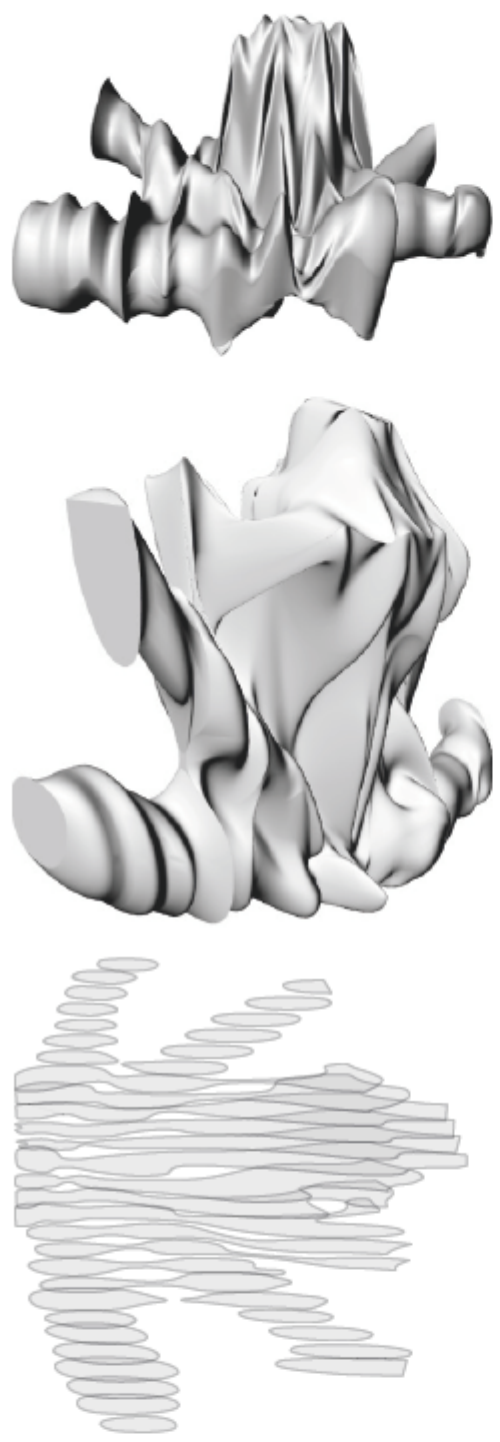

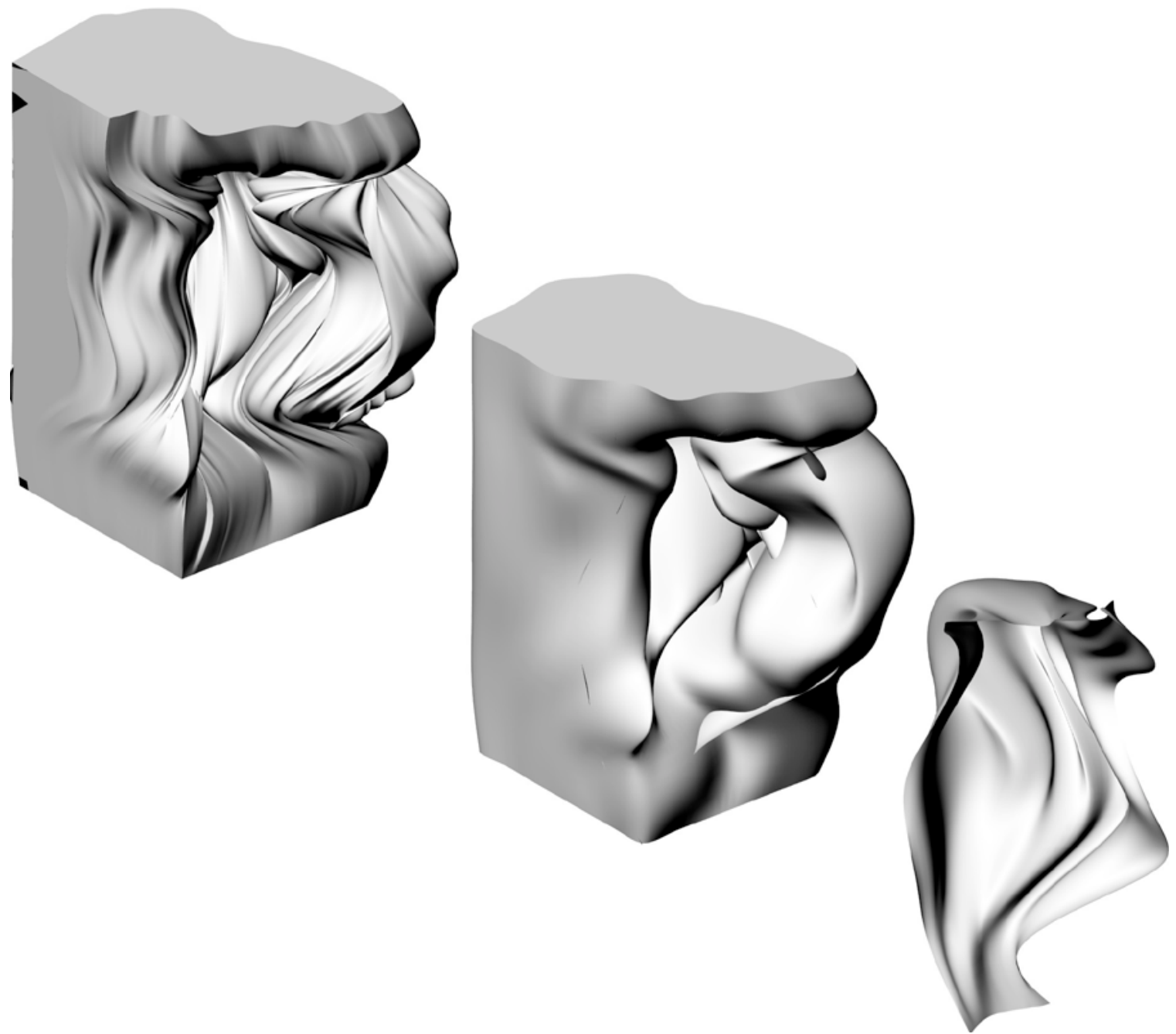


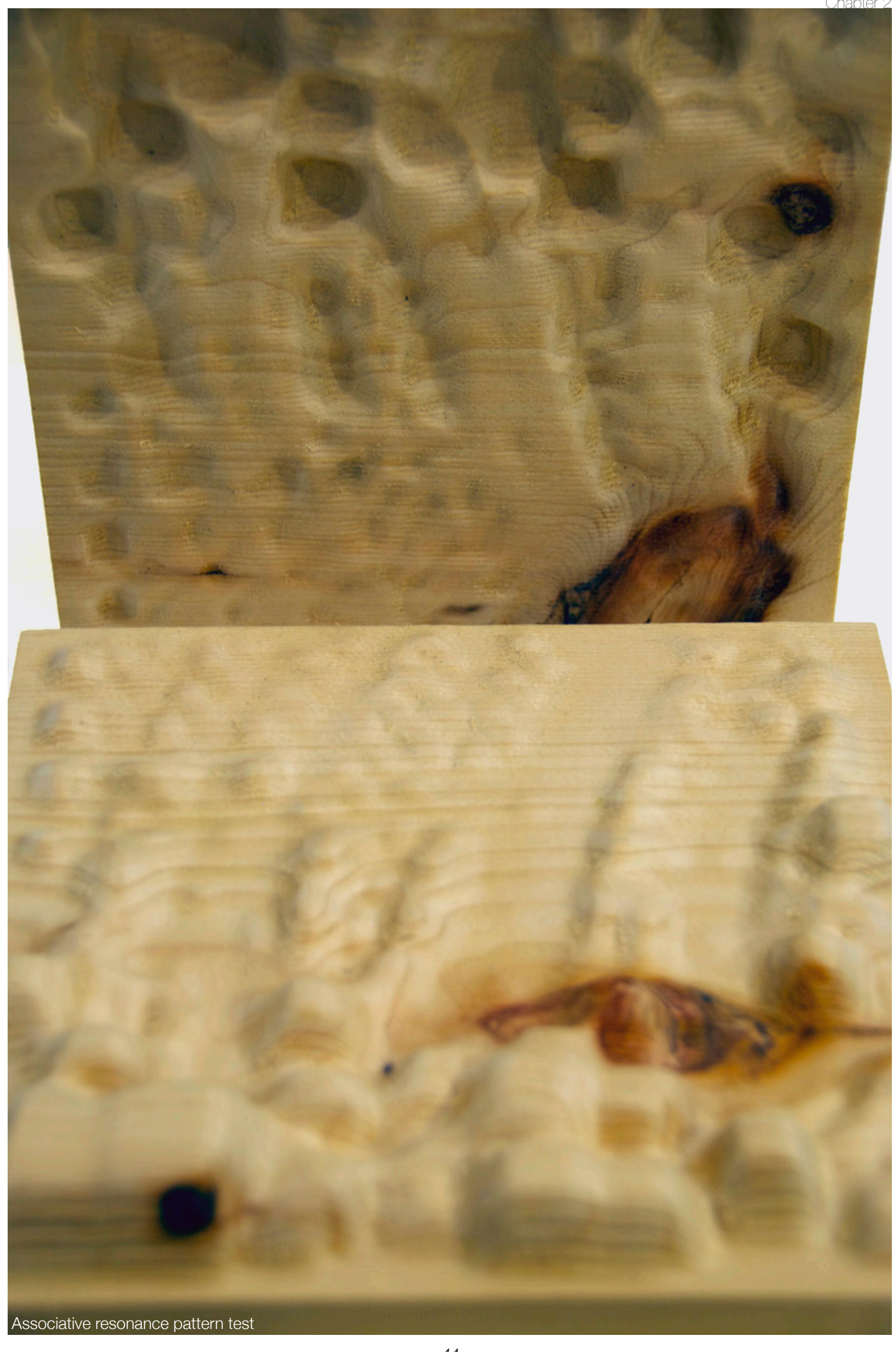


$\log 1$

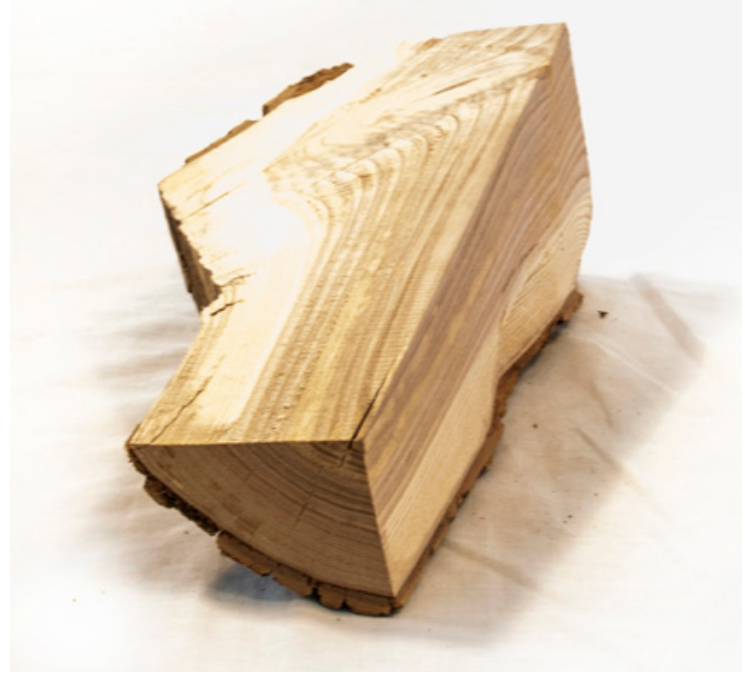

$\log 2$

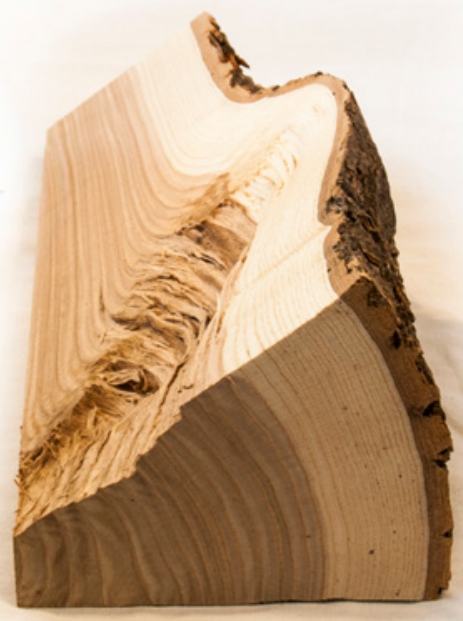

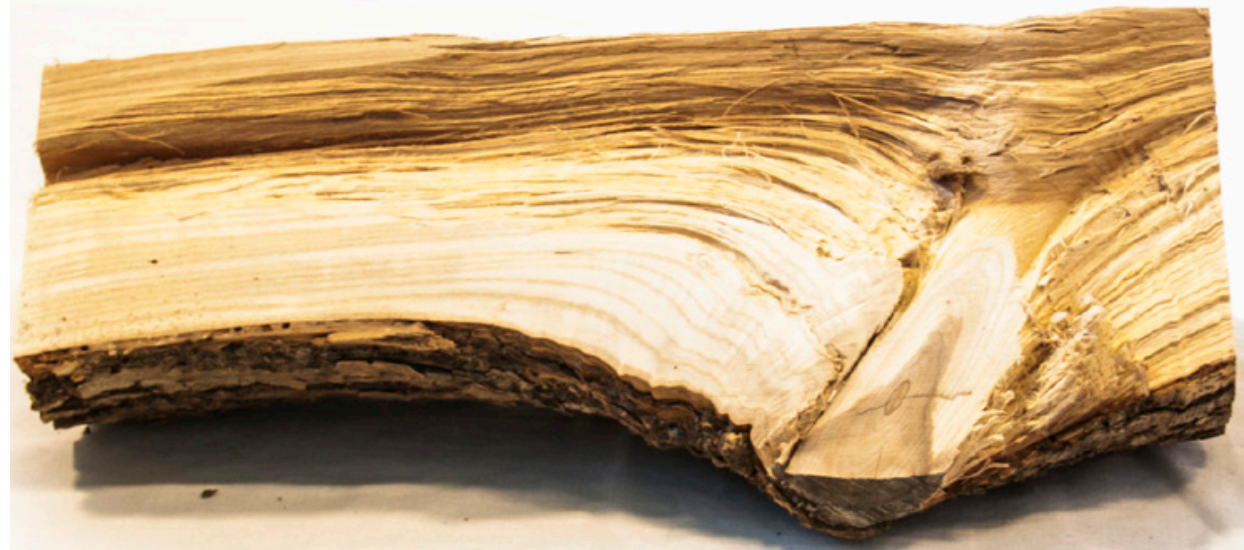

$\log 3$ 


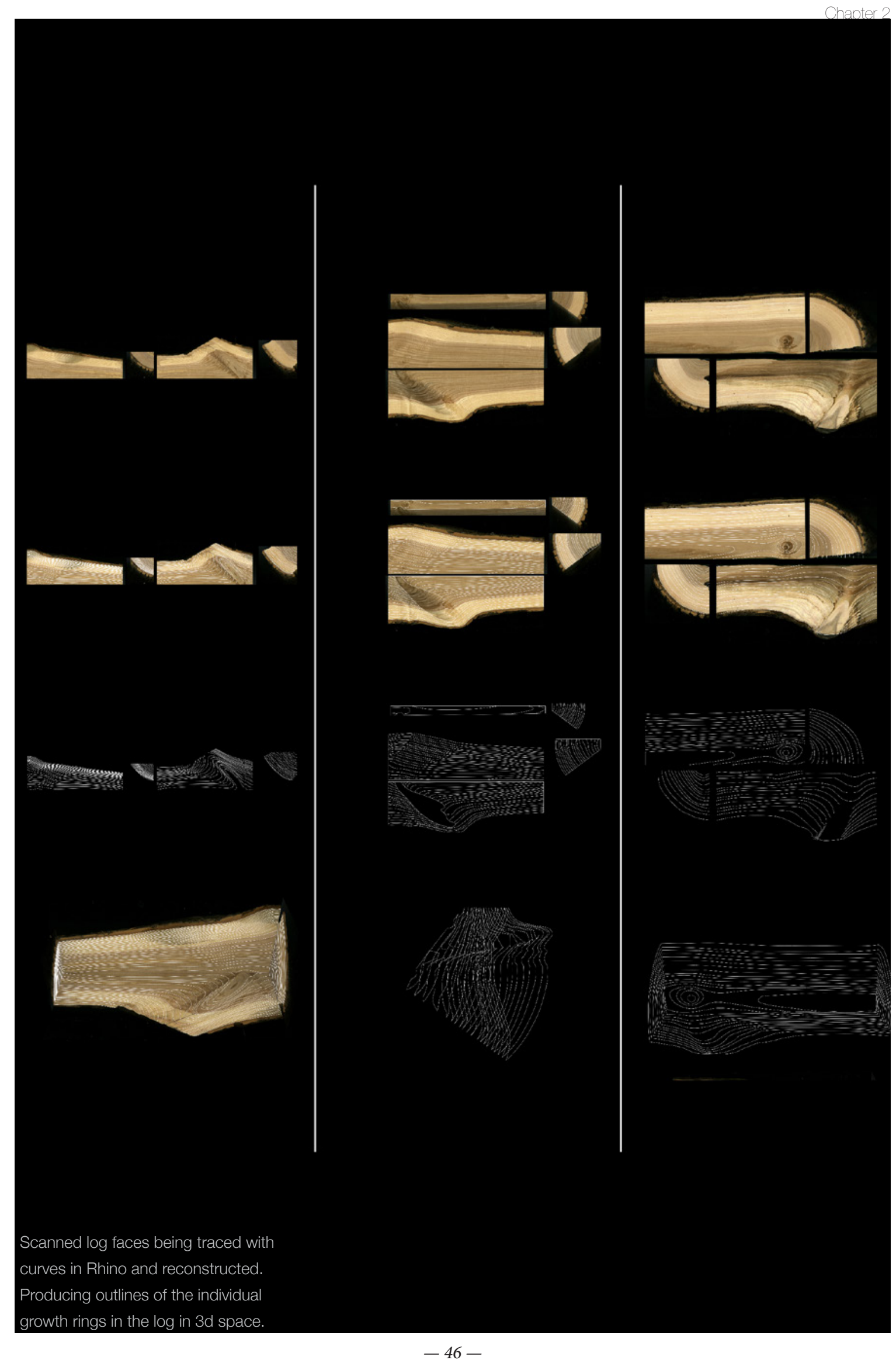




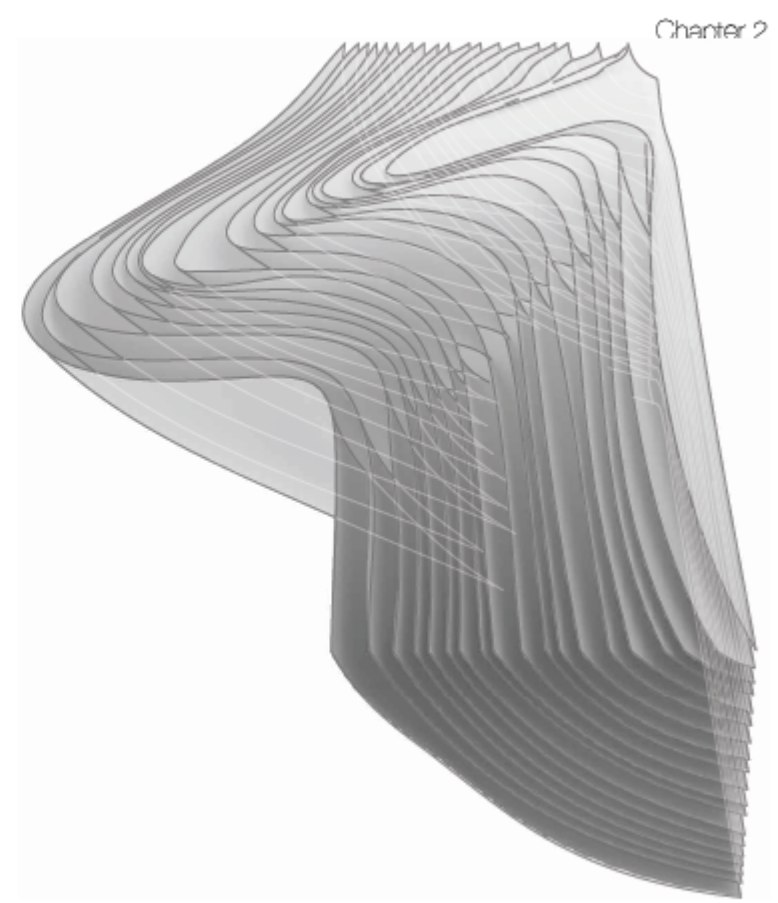

Growth Rings Isolation 1

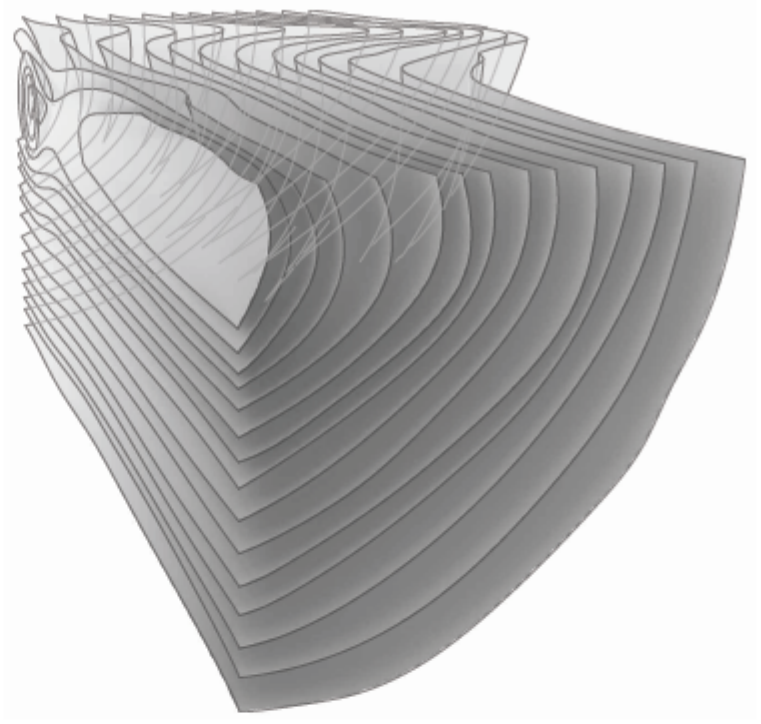

Growth Rings Isolation 3

These diagrams show the inferred growth rings through the wood by digitally tracing the growth ring sections from the scans. By planing flat sides and contrasting the fibers, surfaces can be recreated of the individual pieces. 
Chapter 2

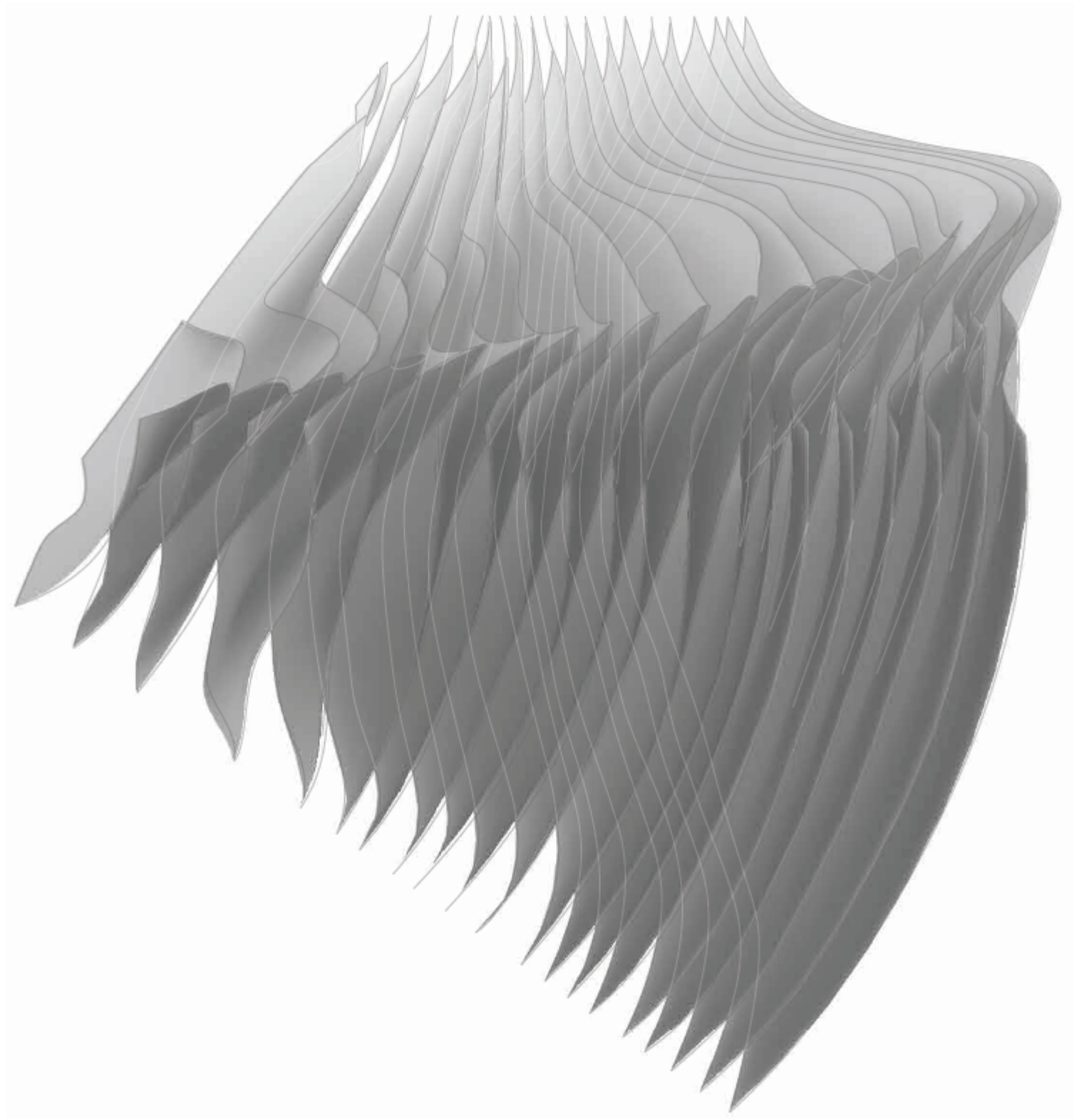

Growth Rings Isolation 2 

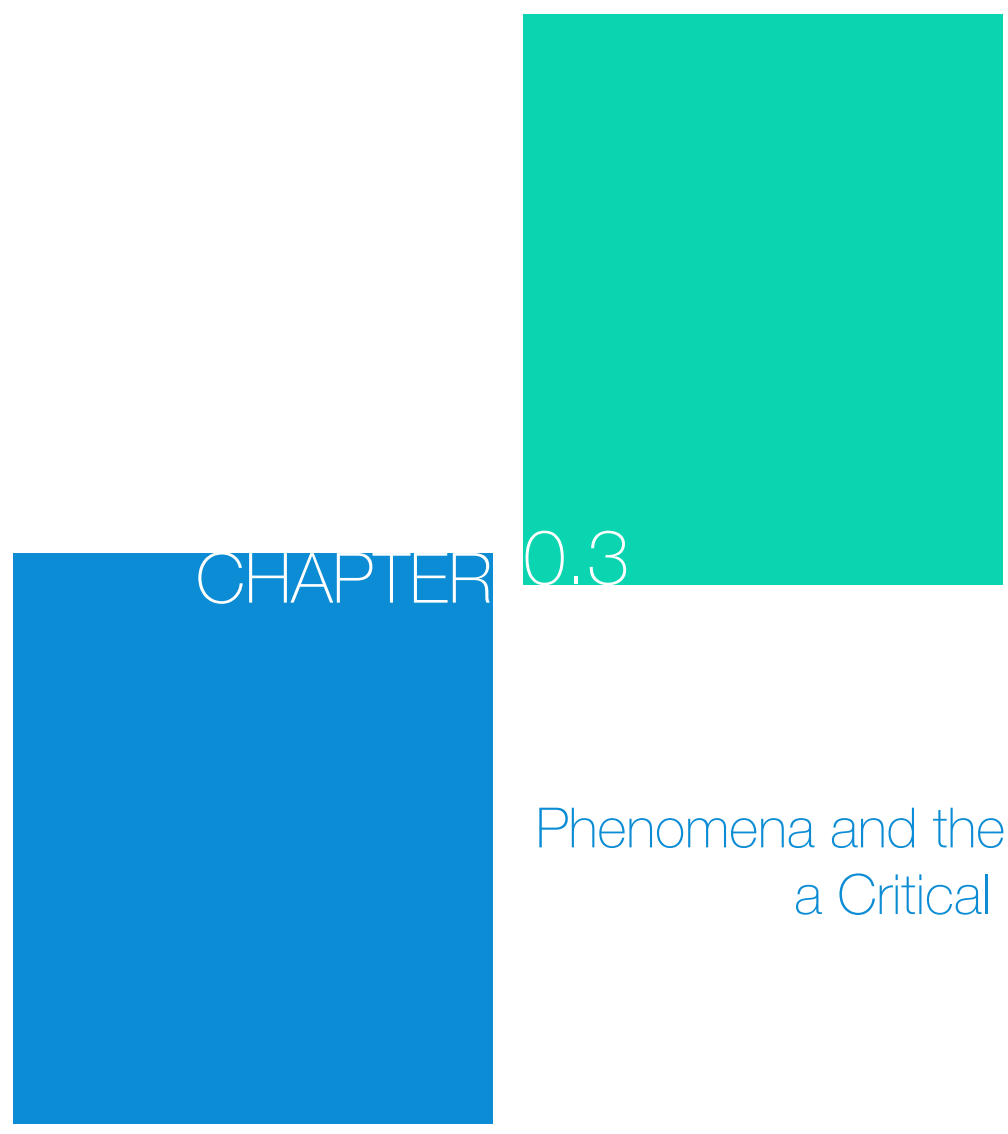

Phenomena and the Existing Condition as a Critical Response 
"Circumstance has no value. It is how one relates to a situation that has value. All true meaning resides in the personal relationship to a phenomenon, what it means to you." _ Christopher McCandless 


\subsection{DIGITAL CONTEXTUALISM}

A key response to the Internationalist movement during the Modern period called for a back-to-basics approach to local architecture and contextual design approaches. Most notably, these included Christian Norberg-Schultz's genius loci and Kenneth Frampton's critical regionalism. Norberg-Schultz argues for an architecture that reacts and synthesizes with the direct site conditions architecturally; his genius loci literally translates to 'spirit of a place.' Frampton advocates for an architecture that rejects centrist ideals and promotes regional design independence as a reflection of that area's character. ${ }^{1}$ Although separate and distinct ideas, NorbergSchultz's 'genius loci' and Frampton's 'critical regionalism' share similar sentiments in their approach to site-based contextual design. Both acknowledge the importance of an identity in the process of design and building, and this could often be construed in their writings as a type of vernacular tradition inherent in a localized context. These determinant factors played a role in their development of a regionalist approach to character; they were localized because they had to be. In this sense, "character" describes the localized visual, formal and material culture. This character spoke directly not only to the lives of their inhabitants, but the relationship to the direct effect that natural phenomena applied to their lives: "In pre-industrial settlements, one had to understand and go with the natural

1 Kenneth Frampton, Modern Architecture: A Critical History (London: Thames \& Hudson, 2007), 327. 

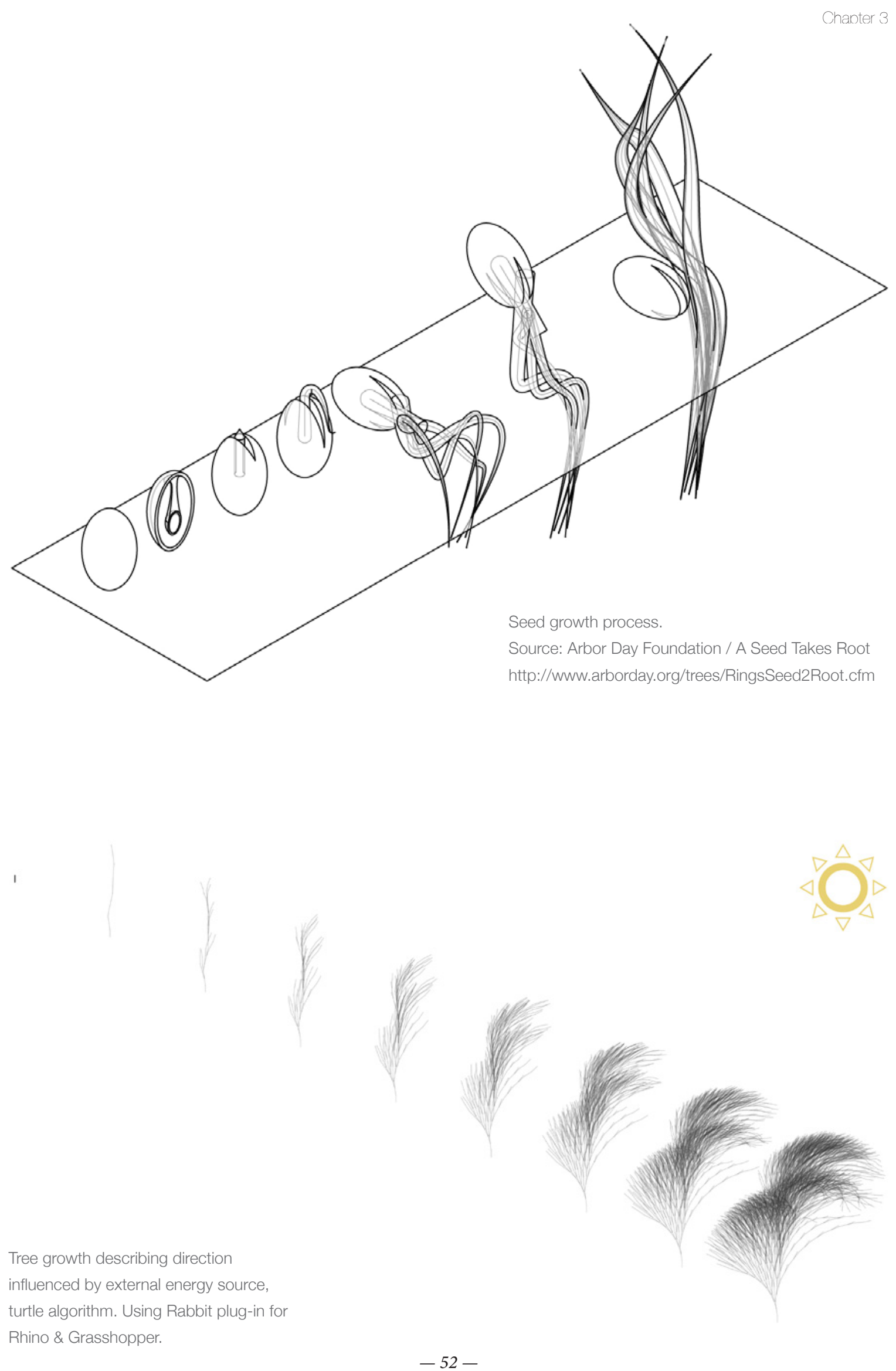
flow. There wasn't the technology to do otherwise. If there was a river, one built along its sides and tried to use it for trade (London). If solar radiation was too intense, then streets were narrow to minimize the surface area exposed to it (Fez). If water was scarce, then all the surfaces of the settlement would be organized to catch and store rainwater for the dry months (Santorini)."2

This reinforcement of the need for a vernacular 'inherent' functionalism could be construed as similar to contemporary function - a turn away from Modernism's idealized-Functionalism, which was reductive to the point of absurd banality. To the contrary, "any study of vernacular settlements will uncover this direct relationship between the form of the unbuilt site and the formation of the built fabric." 3 In its own idiosyncratic way, the vernacular could at times be more functionalist than actual Functionalism. But while vernacular architecture responded to a direct need with the available materials at hand, technological advances have dated many of these due to evolved performance standards. By extension, in many cases a return to purely vernacular motifs tends to stray toward stylistic kitsch due to a crutch on the sentimental image of that architecture. Because of this reduction, "the work appears sentimental or kitsch as our mental awareness glides too easily into the suggested illusion." 4 This suggests that enabling a material kitsch is reductively grounding to the viewer, and is a key problem of the Norberg-Schulz

\footnotetext{
Hagan, Digitalia, 116.

Ibid.

Pallasmaa, Embodied Image, 95.
} 
Anatomy of branched growth
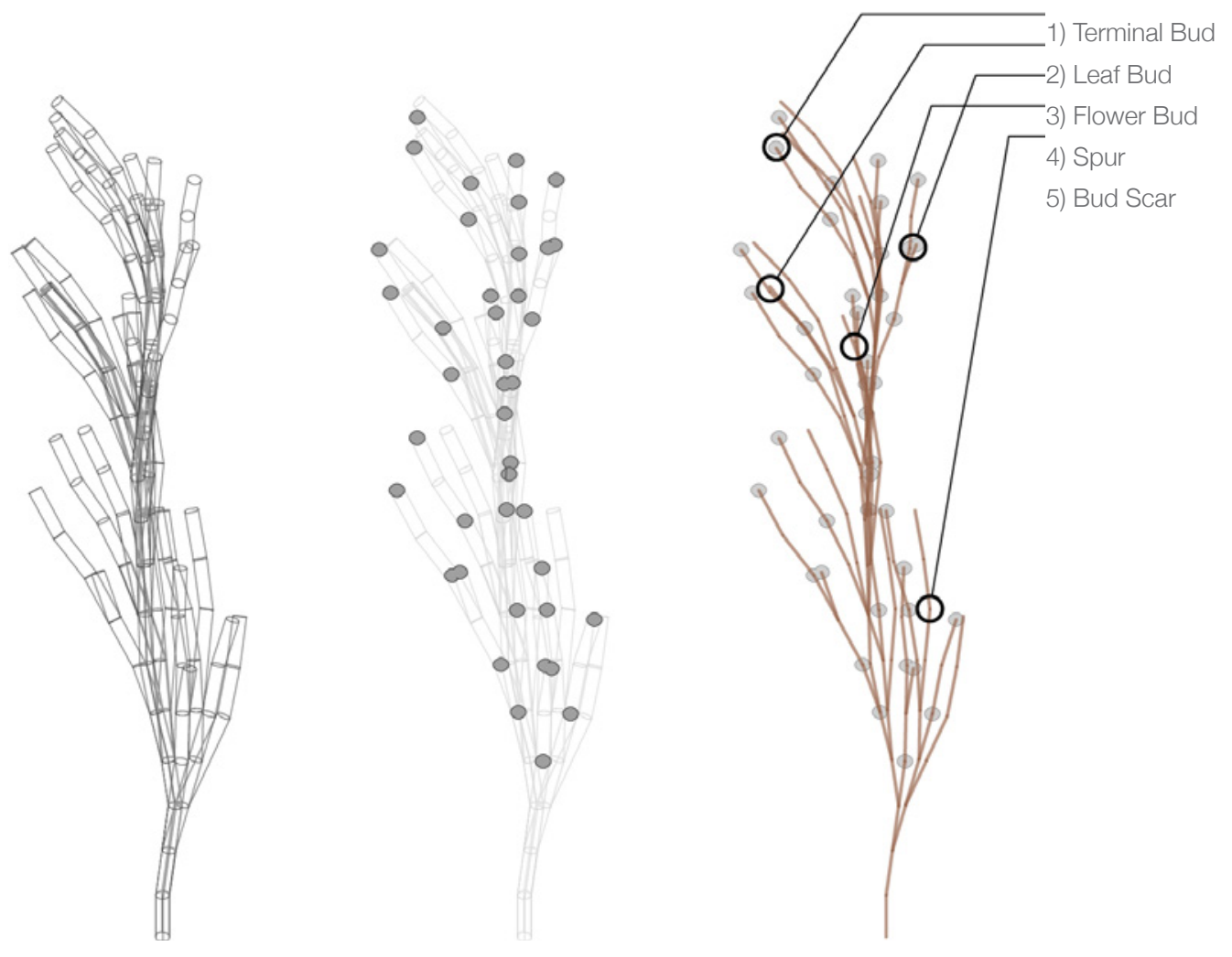

Source: Oregon State University. Extension Service, Lane County. http://extension.oregonstate.edu/ linn/sites/default/files/pruning_pub_ handout2012.pdf 
methodology for 'place.' It is also one that the digital is able

to circumvent simply due to the fact of its immateriality.

Digital methods have downsides, particularly the need to "give expression to the computation without simply representing the datascape." ${ }^{5}$ In these cases, as a contrast to the vernacular kitsch, "when the reality of matter or execution dominates, the work tends to appear crude and unarticulated, and it seems incapable of evoking a credibly imaginary world."6 Pallasmaa suggests therefore that an embodied material condition will produce a more powerful architecture when it is able to produce within tension between these two sides. This suggests the act of getting inside the makeup of the material instead of passive observation.

To this end, Pallasmaa and his associative writing contemporaries are often in praise of a critical regionalist approach of contemporary practice, which tend to skew towards what could be described as phenomenologistcentric practices such as Peter Zumthor, Glenn Murcutt and Brian MacKay-Lyons. With the latter, MacKay-Lyons' working method has been coined as "plain modern," a successful interpretation of Modernism's ideals coinciding with subtle assertions of the Nova Scotia vernacular that avoid the tropes of kitsch. To achieve this he has developed a systemic working method for a site-based contextualism. The "Three F's for an Architecture of regionalism" can be summarized as:

5 Trevor Patt, “Taipei 2.0.2: Computation and the Urban Generic," In Highrise Shuffle (Finland: Multiprint Oy, 2011), 114.

6 Pallasmaa, Embodied Image, 95.

7 Quantrill, Plain Modern, 27.

8 Quantrill, Plain Modern, 32. 

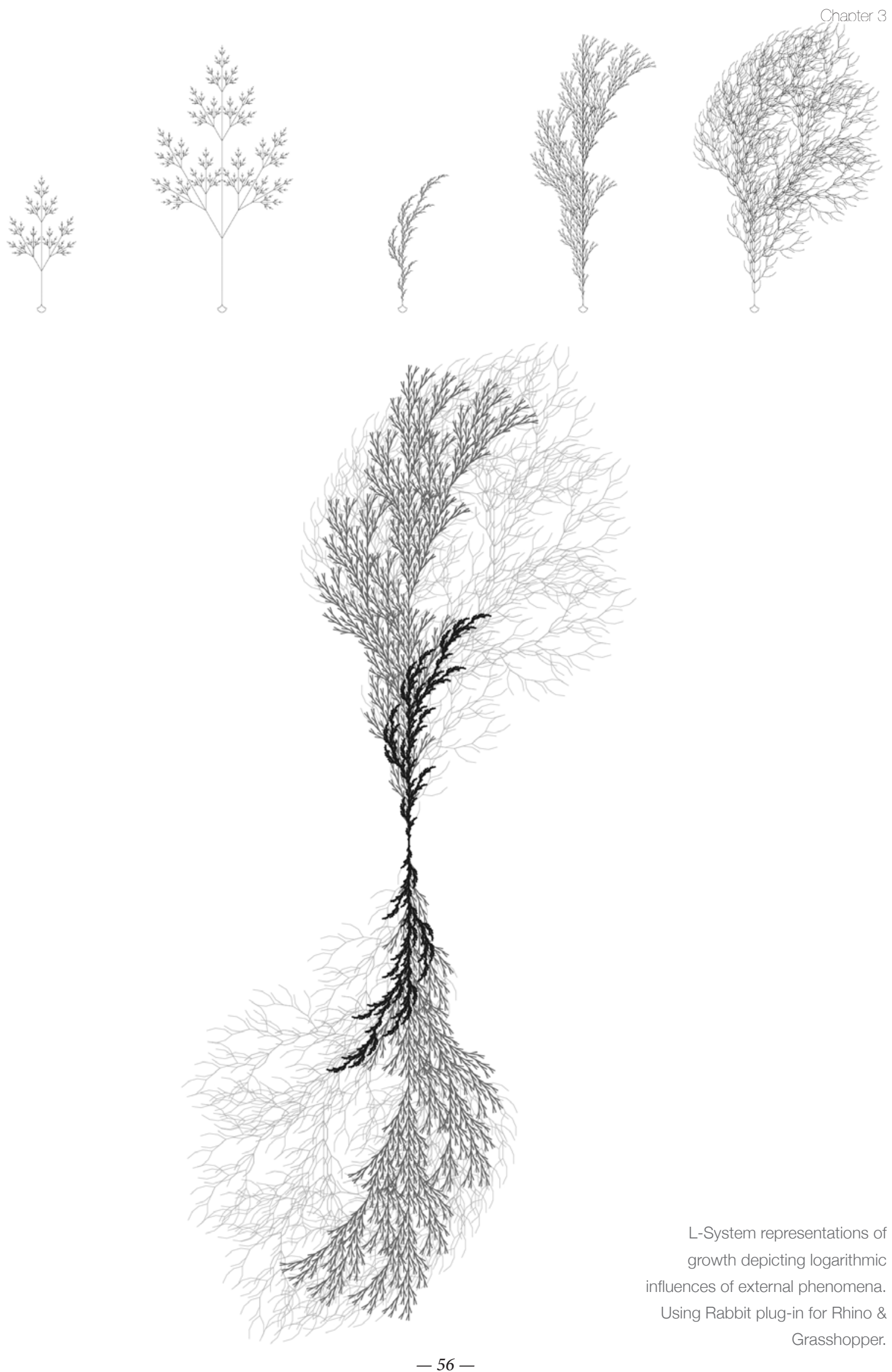

L-System representations of growth depicting logarithmic influences of external phenomena. Using Rabbit plug-in for Rhino \& Grasshopper. 
Fitting as a metaphysical method of siting in the

landscape as a reaction to the established characteristics already there,

Framing as a way to develop a skeletal representation of the conceptual idea without establishing hard boundaries,

Forming as a way of establishing the overall shape to reinforce the idea of the project. ${ }^{9}$

While he remains effusive in his regard for the computer as a useful and necessary part of the production process, Pallasmaa conspicuously refrains from discussing any contemporary firms that have incorporated cuttingedge design technologies into their workflow. This is unfortunate, as much of the praise is for works that have incorporated a tension between the digital and physical. The vernacular kitsch is simply a stylistic device, but the push towards its use as a fundamental, broader symbol of regionalism represents a place-making device, independent of any personal preference of best-practice. To build on MacKay-Lyons 'plain modern,' creating contemporary place should seek a 'plain digital.'

Norberg-Schulz describes a similar key divergence between that of the Vernacular Genius as a literal interpretation of the locality of place that is circumstantial (farms and villages) as opposed to the Urban Genius as a broader interpretation of the local genius in a symbolic transposition (squares and public structures). ${ }^{10}$ This is 


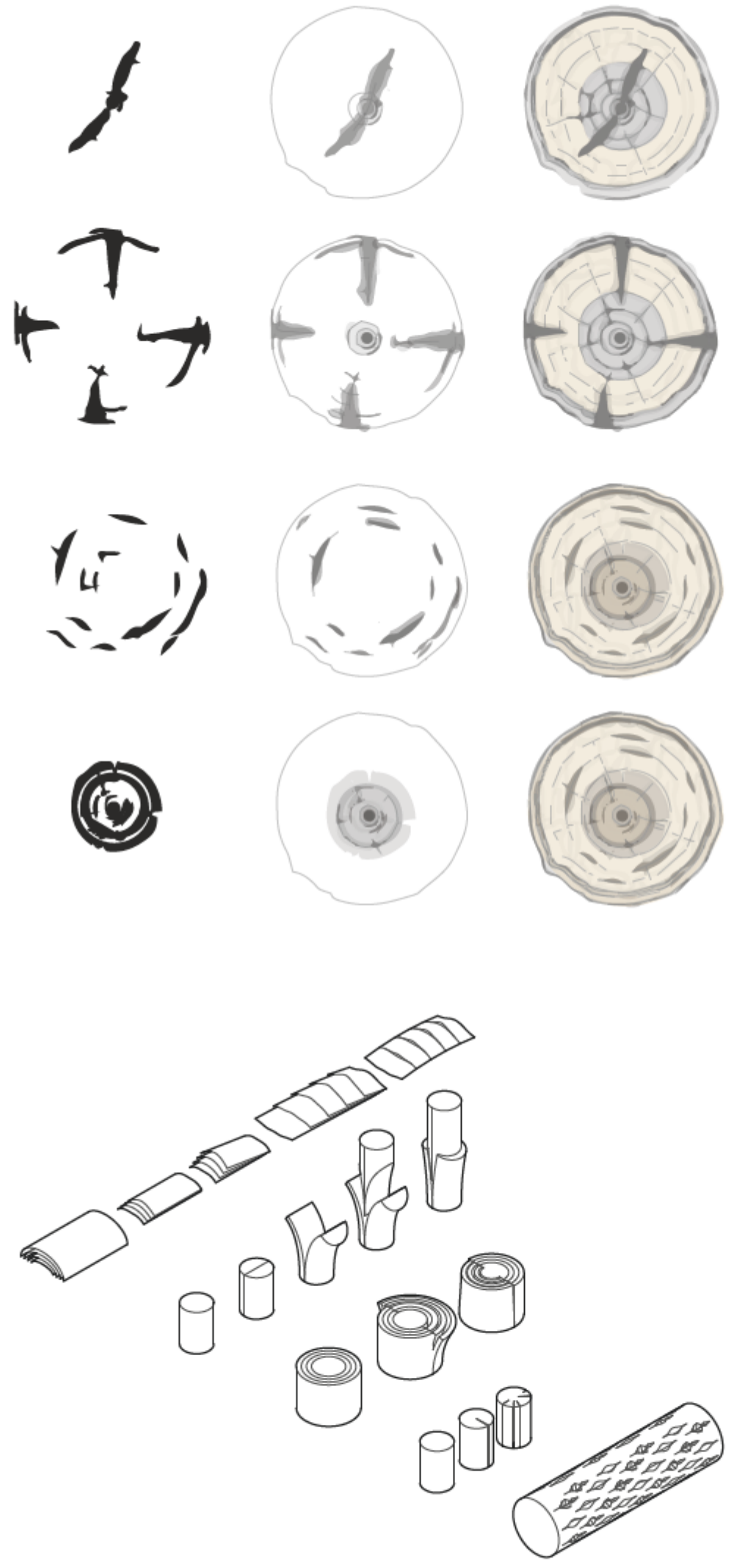

Heart Shake
Internal splitting, or honeycombing,
radiating from the center of the grain.
Perpendicular to the growth rings, with
the adjacent fibers separating from one
another parallel to the main tre日 axis.

Surface Checks

Cracking around exterior of log in the radial direction (perpendicular to rings), splitting caused by force of drying shift.

\section{Ring Shake}

Splitting in parallel tangent along tre日 rings. Caused by lateral forces during growth, or heavy lateral turbulent forces after decay (drift wood). Can also be caused by exterior hardwood pressing into softer center wood. It is the result of tension build-up on one side versus the compressive force on the opposite side of the tre $\theta$ and the cracking betweөn the most tense rings.

Source: Kumal Buhsal, "Defects in Wood." http://forestrycomponents. blogspot.ca/2010/03/defects-in-wood. html

Deteriorating wood conditions.

1) Tension build-up

2) Grafted trunk

3) Ring shake (Int. and Ext.)

4) Singular surface checking

5) Broad surface checking 
corroborated by Frampton's statement that he does not

intend Critical Regionalism "to denote the vernacular as

this was once spontaneously reproduced... but rather to

identify those recent 'schools' whose primary aim has been

to reflect and serve the limited constituencies in which they

are grounded."11

To ignore the face value appliqué in favor of an

understanding rooted in phenomena similarly represents

a place-making device for a new genius loci. Natural

phenomenon of a distinct area represents a rejection of

centrist ideals in favor of a regional architectural working

methodology that reacts and synthesizes to its component

parts and surroundings. Interpreted this way, vernacular

$11 \quad$ Frampton, Modern Architecture, 317.

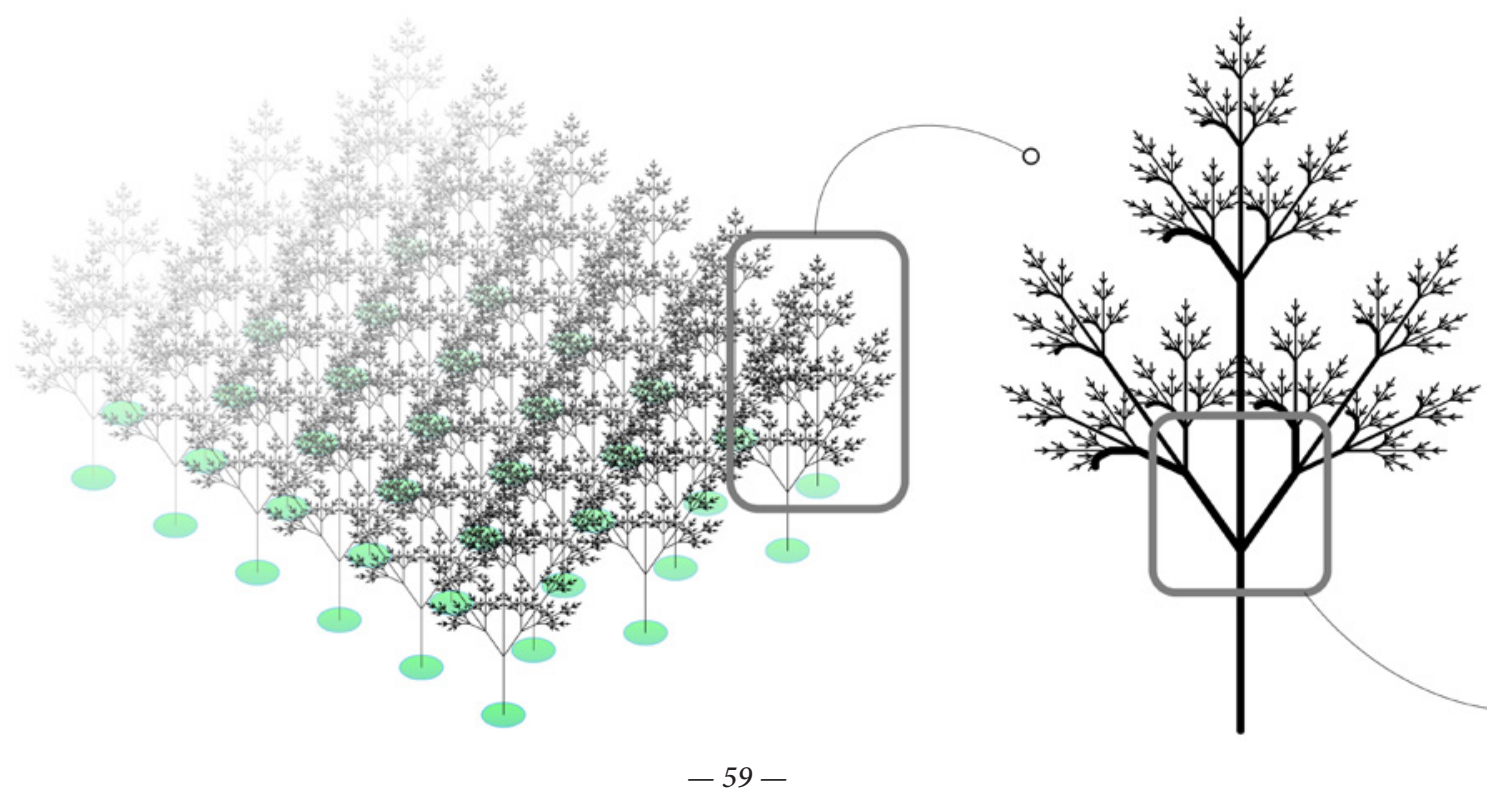


is more functionalist than Functionalism. In this sense it

becomes a truly responsive system. After all, what could be more phenomenal than actual phenomena?

\subsection{IMITATIVE ENTITIES:}

\section{BIOMIMICRY AND PHENOMENA}

This thesis is explicitly exploring the phenomena of growth, in how it effectively responds to local context. While there is some overlap in their respective themes, it should be noted that there exists a clear distinction between 'imitation' and 'biomimicry' in architecture. The term biomimicry has gained traction since the introduction of Buckminster Fuller's geodesic domes, and has slowly

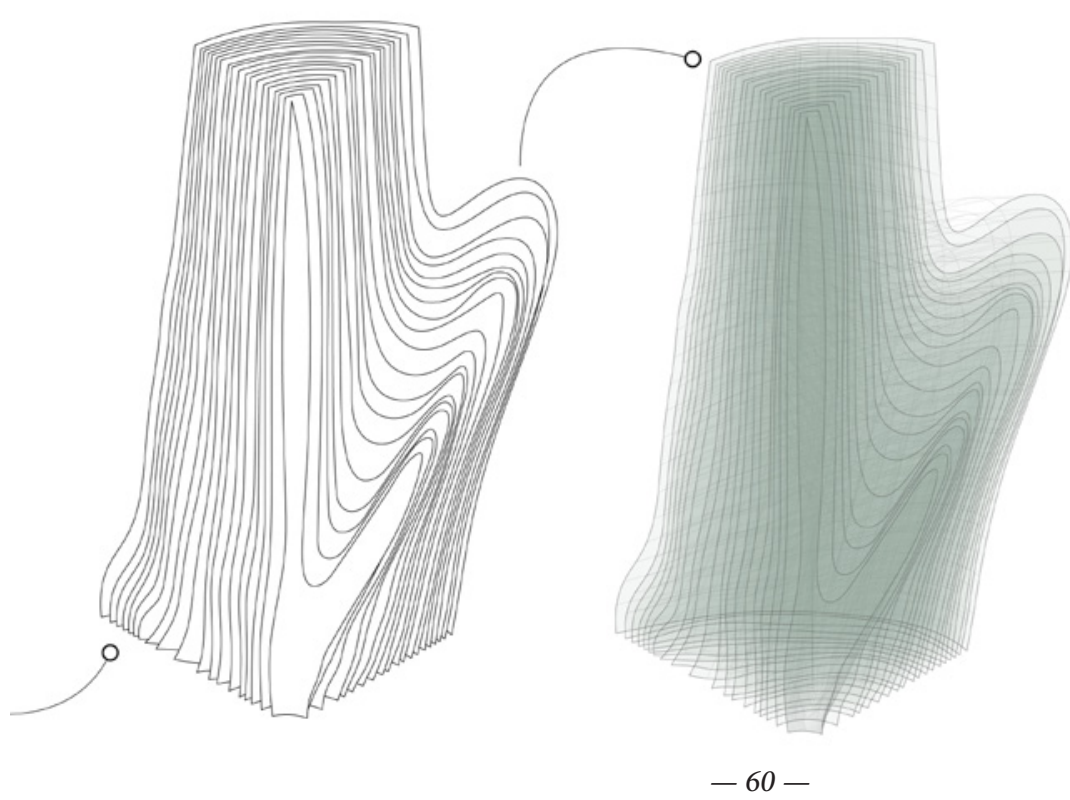

LL - Forest of localized systems. LR - Growths within individual system. $\mathrm{RL}$ - Growth ring evidence. $\mathrm{RR}$ - Digitally rebuilt localized condition. 
entered the rhetoric of contemporary discourse.

While it would be unreasonable to outright reject the idea of a biomimetic architecture, there are some key fallacies in its discourse. Most notably is rhetoric of architectural biomimetics instead of actual biomemises - just because something looks biological does not inherently make it so. Organic form or paneling does not automatically produce biomemises. A turn instead towards appropriating the natural systemic occurrences within a growth offers a more pragmatic way of thinking about architecture's relationship to biomimesis. This requires focusing on the forces producing the result rather than the result itself, and what the architectural implications of those are.

The main issue with a biomimetic process appears to be its obsession with skinning and façade systems. It often appears that a biomimetic architecture appropriates a reactive process strictly in the sense of how it reacts in physical connection with the elements around it. It is an internal method, and as Derix points out, "User-centric simulations are generally post design and driven by the need for evidence reporting, such as pedestrian simulations for fire egress... [However, the] less visible computational agenda has asked exactly those questions of the correlation between spatial dynamics and form." ${ }^{12}$

Therein precisely lies one of the key disconnects in physical and digital design: the predictive methods that can be extrapolated from a digital system are often not 
given the credence they deserve in the physical realm.

"Schmarsow was inspired by the natural sciences and wanted to systematize architecture, while the organic architecture worked at implementing their concepts on real buildings."13 This may be where a divergence of thought begins to emerge and establish key difference of biomimicry and phenomena. To define imitation in the context of natural architecture is to appropriate their the rather than the product. A system can be synthesized and applied to new contexts. Therefore biomimicry can still be generative, however, the biomimetic process is in fact searching to push towards an observed ideal (or a parametric space of an observed ideal). The method of appropriating a phenomena proposes instead a predictive methodology from the observed that can be instilled to produce an unknown end, or may not even have an end at all. This itself is what constitutes a system which, as a model, is more suited to a node-based/networked approached. 


\subsection{INVESTIGATION II: PREDICTIVE PHENOMENA}

Investigation I showed a simple understanding of recreating evidence of existing phenomena. To expand on this, how can the same tools be used to incorporate further complexity through the returnable editing process that is inherent in Grasshopper?

This was explored in two ways. Firstly, understanding the way that a growth expands within the context of a an already compressed and enclosed space. This created a set of generative drawings by simply using the field component at its most basic level. These growths, as emergent entities inside of an established core, denote the 'node,' and signify pressure-points that exert catalyst influences to their immediate and extended surroundings.

By understanding the way that a knot growth occurs, the second analysis broke down the process into its basic formal components and defined them in Grasshopper geometrically by their relationships. From this set of conditions, a growth can start to be predicted/generated within the model as defining a parametric space. This process can then be appropriated to other instances and influence a broader set of conditions as opposed to only being situated on the single object. Those results can then be fabricated to produce a digital crafting of the 'ghosted knot' where the physicality reality once existed previously.

Up to this point, analysis has shown where the growth phenomena has already traveled. By understanding the way that the micro-movement of tree structures are 
moving, conditions can be set up to manipulate the vector

flows of the growth in anticipation of manipulating them for specific ends.

It should be noted that while this portion of the study is attempting to find the processes of growth, the general opinion of biologists is that this cannot definitively be done at an elemental level. Pinpointing exactly the generative growth is not possible. However, broader patterns can be found when accounting for surrounding conditions, such as across a larger region of forest. ${ }^{1}$ Therefore accuracy, not precision, is prominent.

1 Andrew Simons, interview with author, 12 Nov. 2015. 

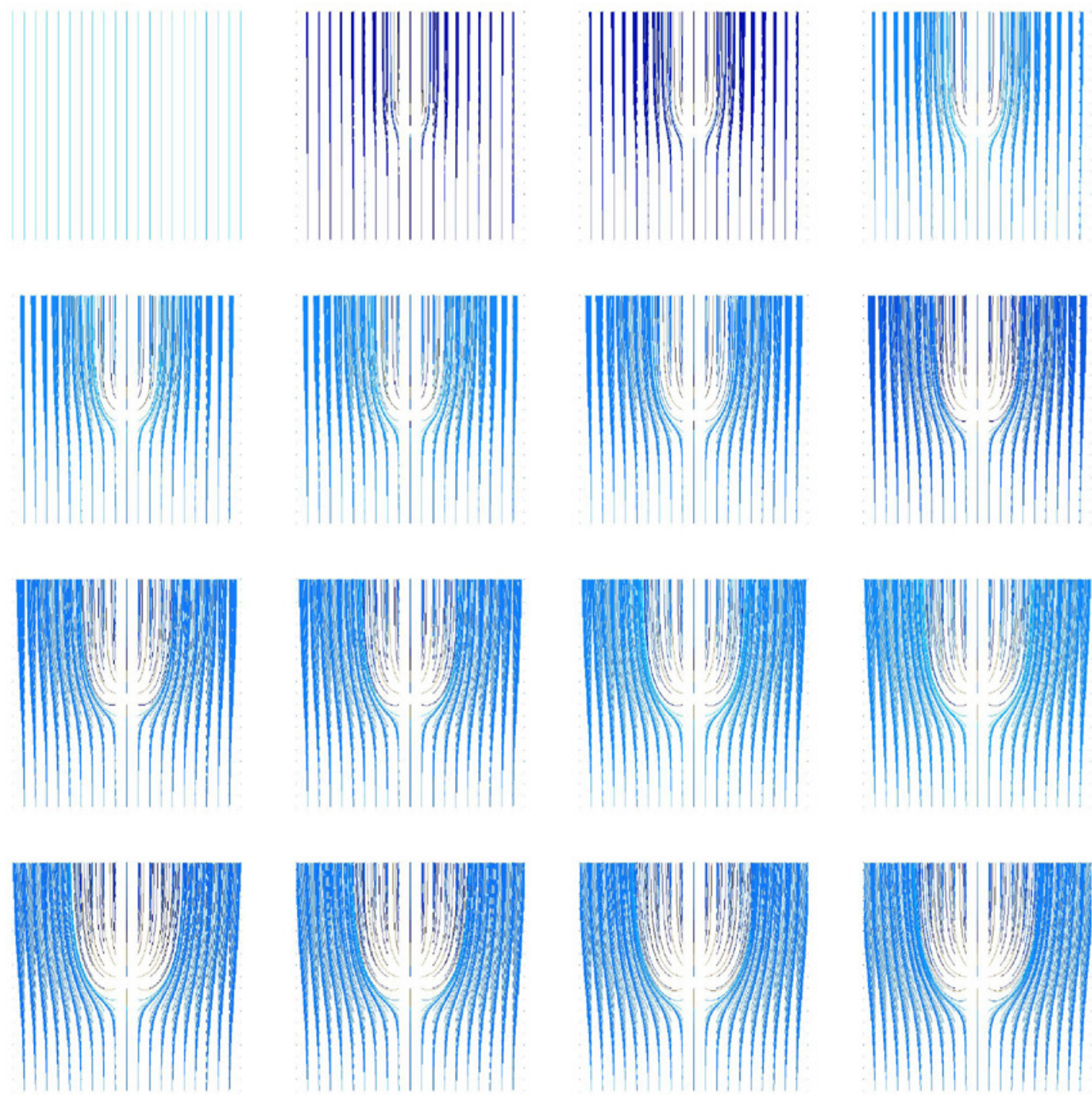

Generated Growth 1, no recursion (describing rates of change and line length of the individual objects with color-coding) 

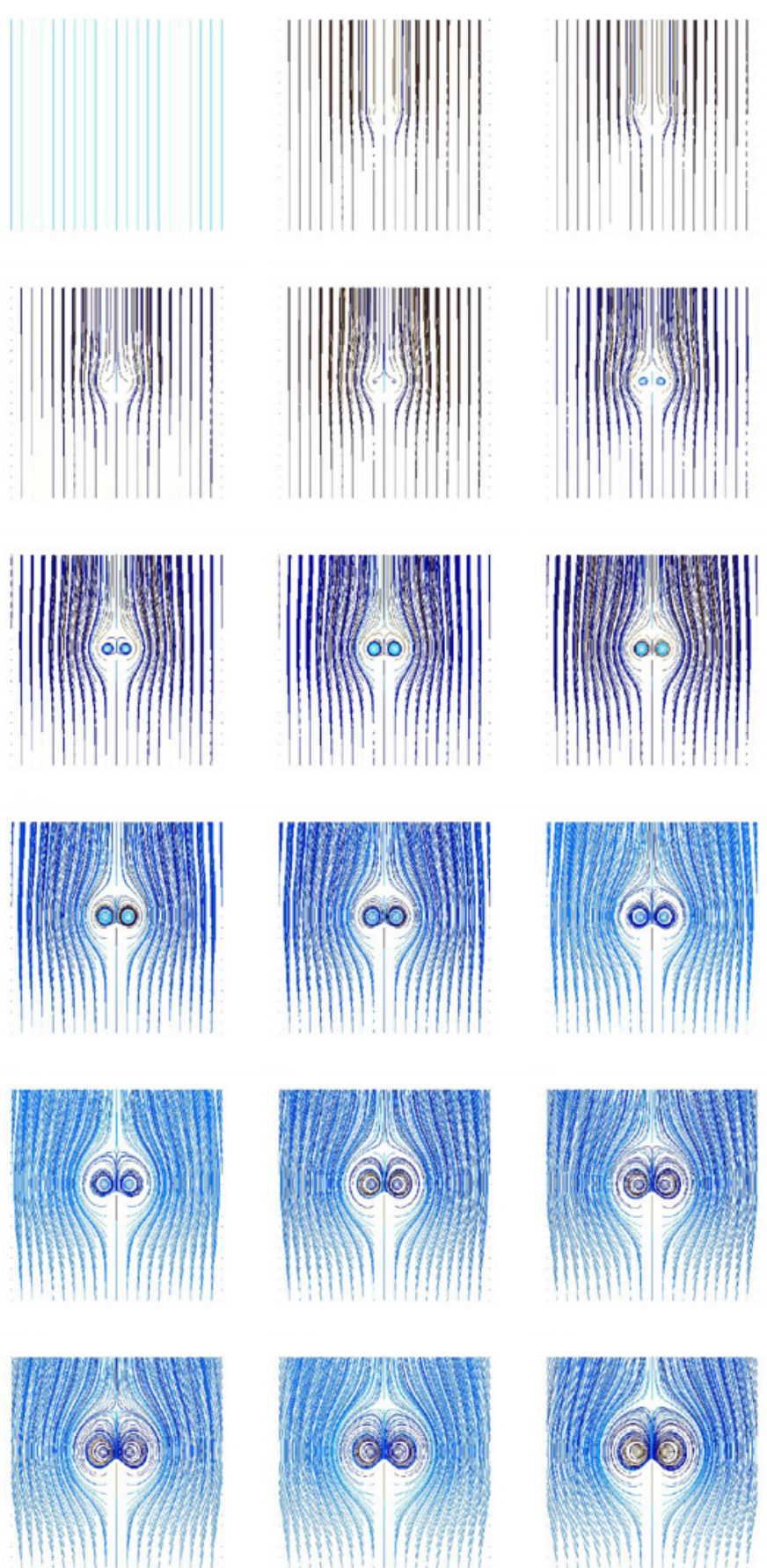

Generated Growth 2, with recursion
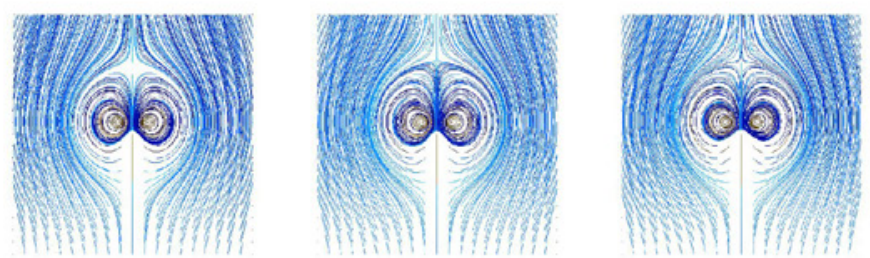
(describing rates of change and line length of the individual objects with color-coding) 


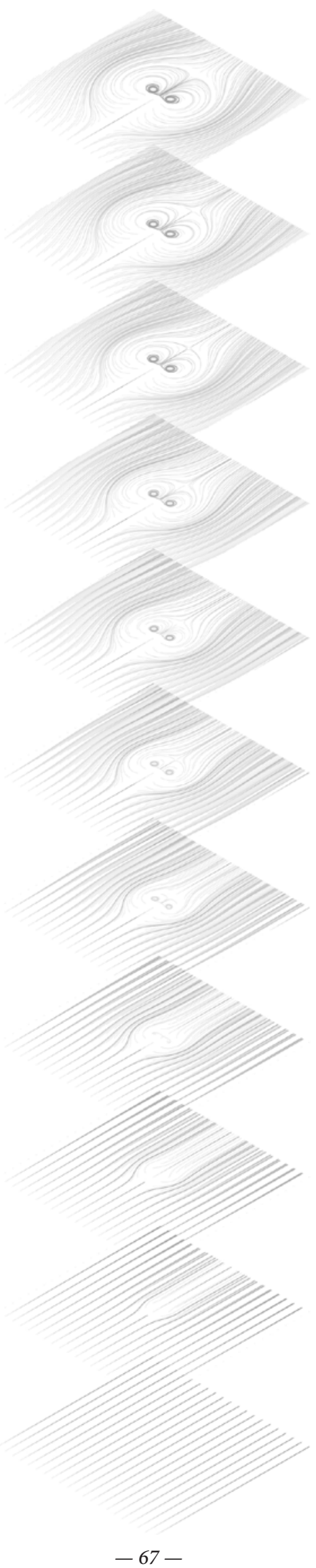




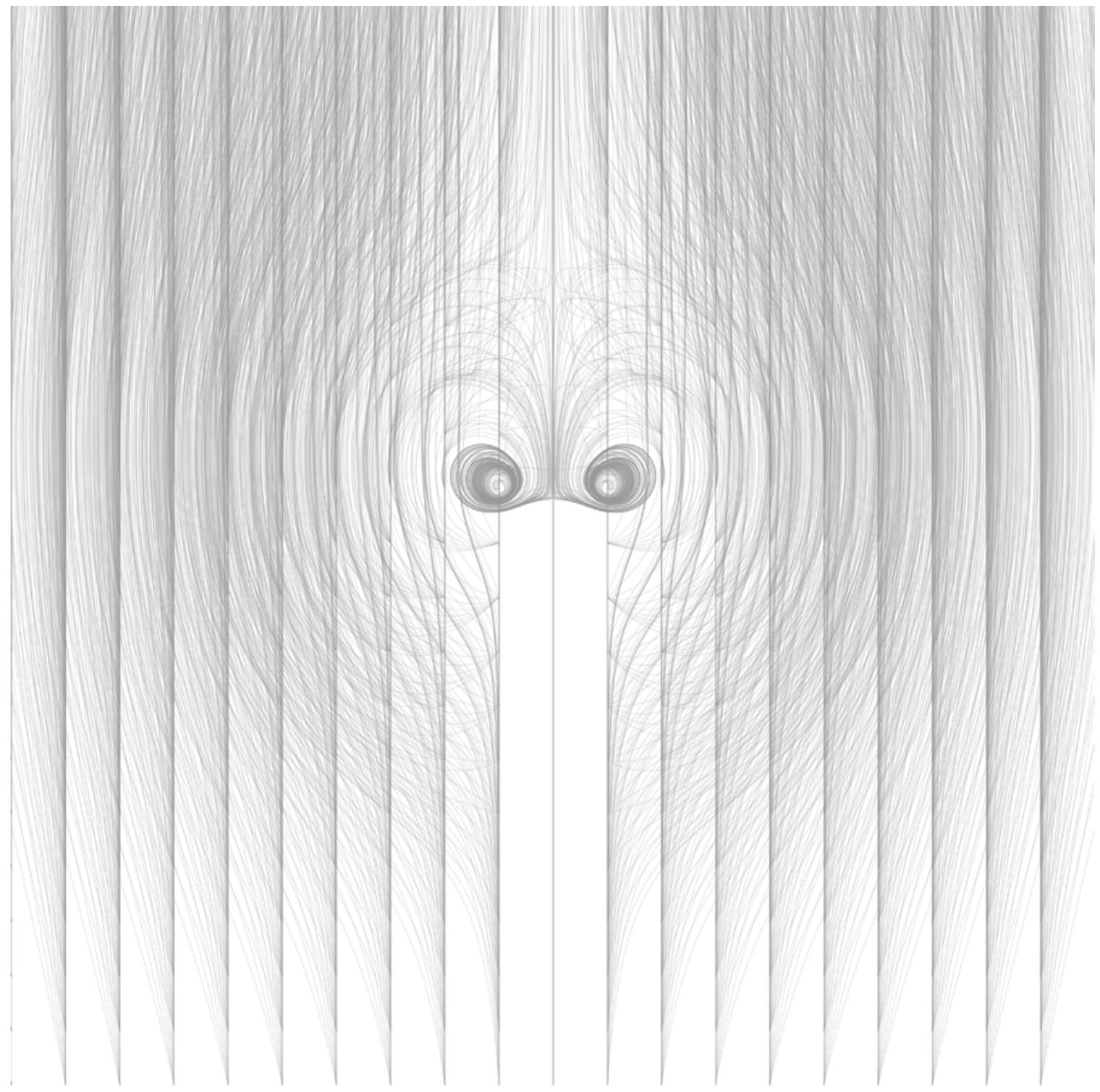


Grafted Branching 1

The Grasshopper scripts (left page) are contrasted with the Rhino model spatial results (right page) as wөll as a description of the definition at that point of the script.

In using Grasshopper and Rhino collectively, the system of growth can start to be defined and generated from a 'ghost' of branched phenomena that once $\theta x i s t \theta d$
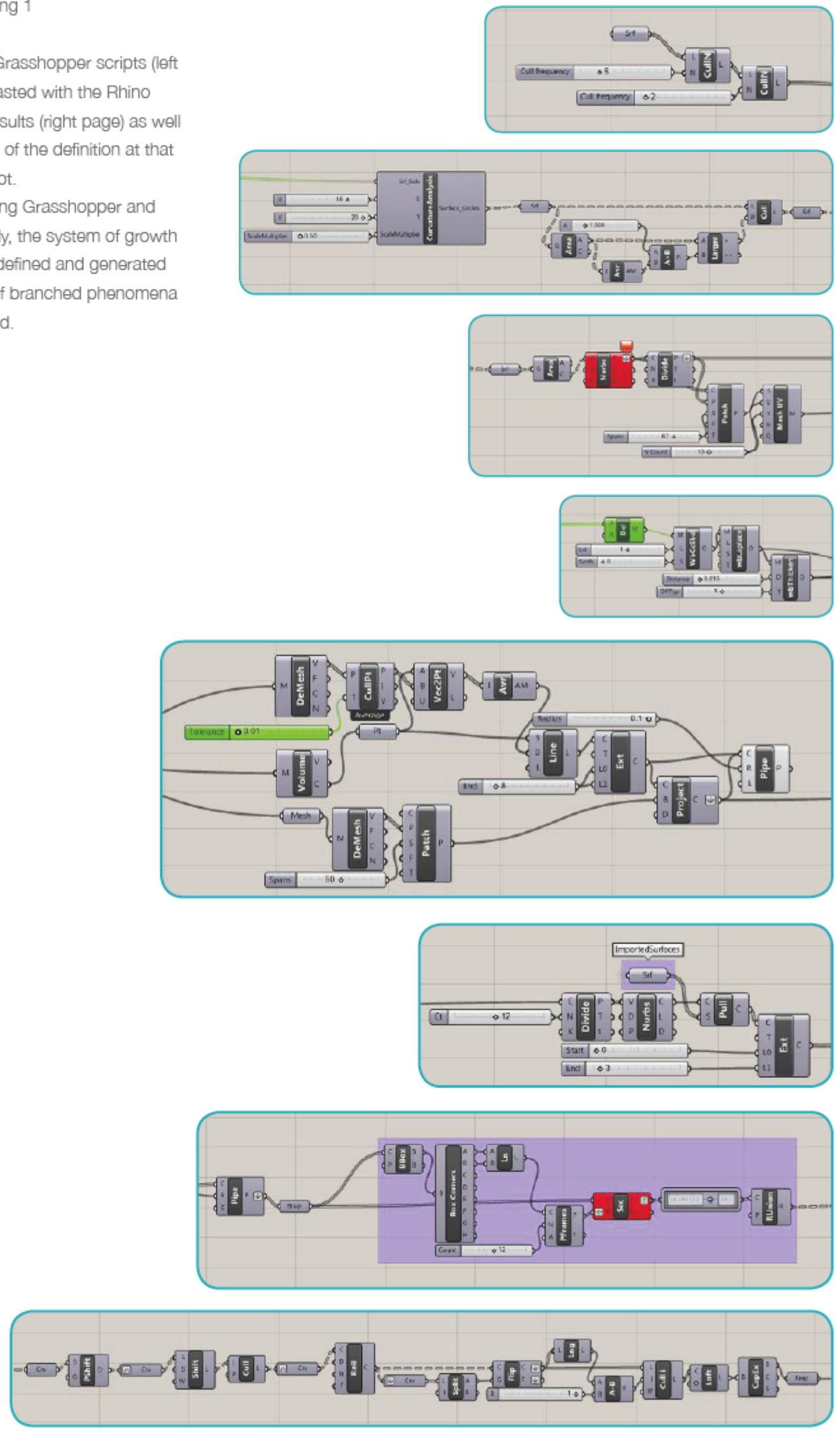

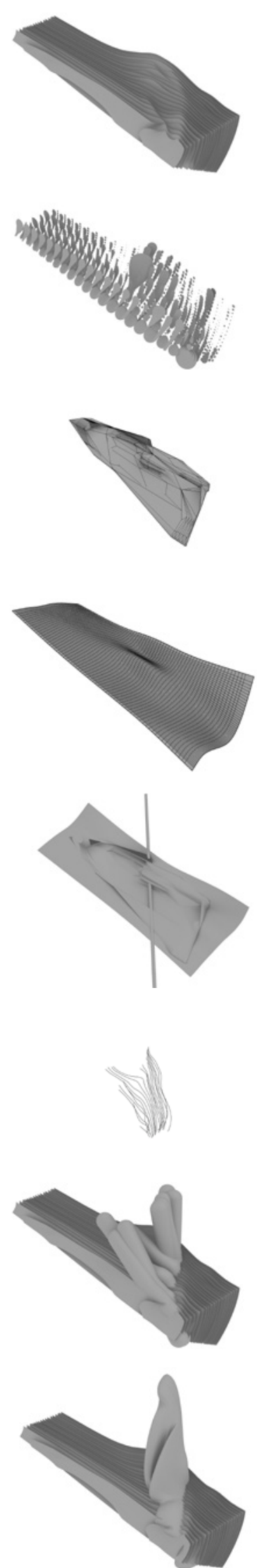

1) The physical act of growth can be reconstructed, as shown in the previous analysis. With a series of surfaces in Rhino, they can be referenced into Grasshopper and subdivided as a set of surfaces into smaller subsurfaces for a more varied analysis.

2) The evidence of a confluence of forces is found by analyzing the rate of change for each subsurface in the ring growths. The result as a rate of change is represented visually by a varying radius of circle.

3) The smaller values are culled out to leave only the points with the highest rates of change remaining (representing large confluence, and therefore growth). These points are connected through a Delauney script to produce a 3D mesh that finds the closest connections between nodes.

4) The delauney mesh is patched into a Nurbs surface and extended to cover the entire digital space of the original growth ring surfaces, showing a visual representation of the force across the object.

5) The mesh (constituting a series of nodes) and the surface (evidence of the parametric field extents) can be incorporated to produce an overall vector force of the growth (shown visually with a pipe) through the analysis space.

6) The growth vector curve is pulled back onto the original growth ring surfaces to show a varied pattern result that influenced in the same manner on the separate instances.

7) The pulled curves are extended outwards and piped to produce a $3 d$ form of the possible parametric space of growth throughout. The curves, however, are still individualized.

8) The $3 d$ forms have contouring sections taking through them, and the results are rebuilt with a smaller number of Nurbs points. The result is lofted to produce a final amalgamated growth knot parametric space and reapplied to the original growth ring surfaces. 

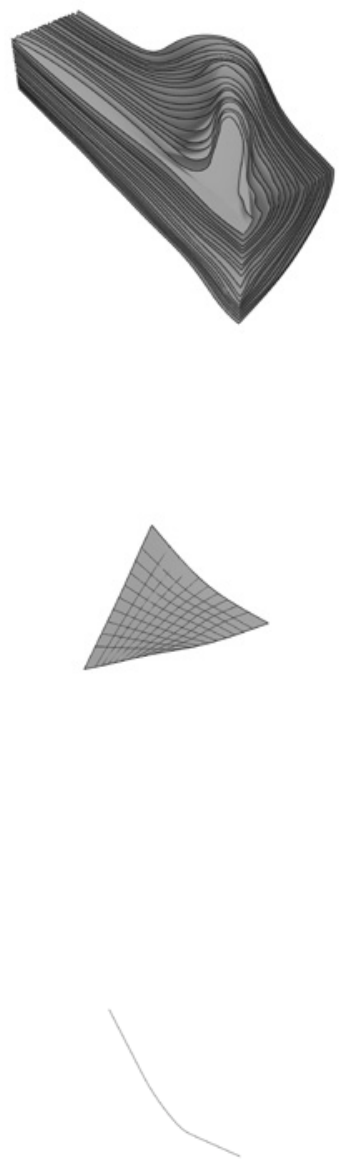

Grafted Branching 2

Because of the broader applicability of using a parametric software to investigate the relationships of growths (both internally and externally) rather than simply reconstructing the existing object, while specific instances may vary, the way that they act does not. The definition remains separate from the particular biases of the singular object. It can therefore be taken and reapplied to another object of a similar family, but with its own unique qualities.
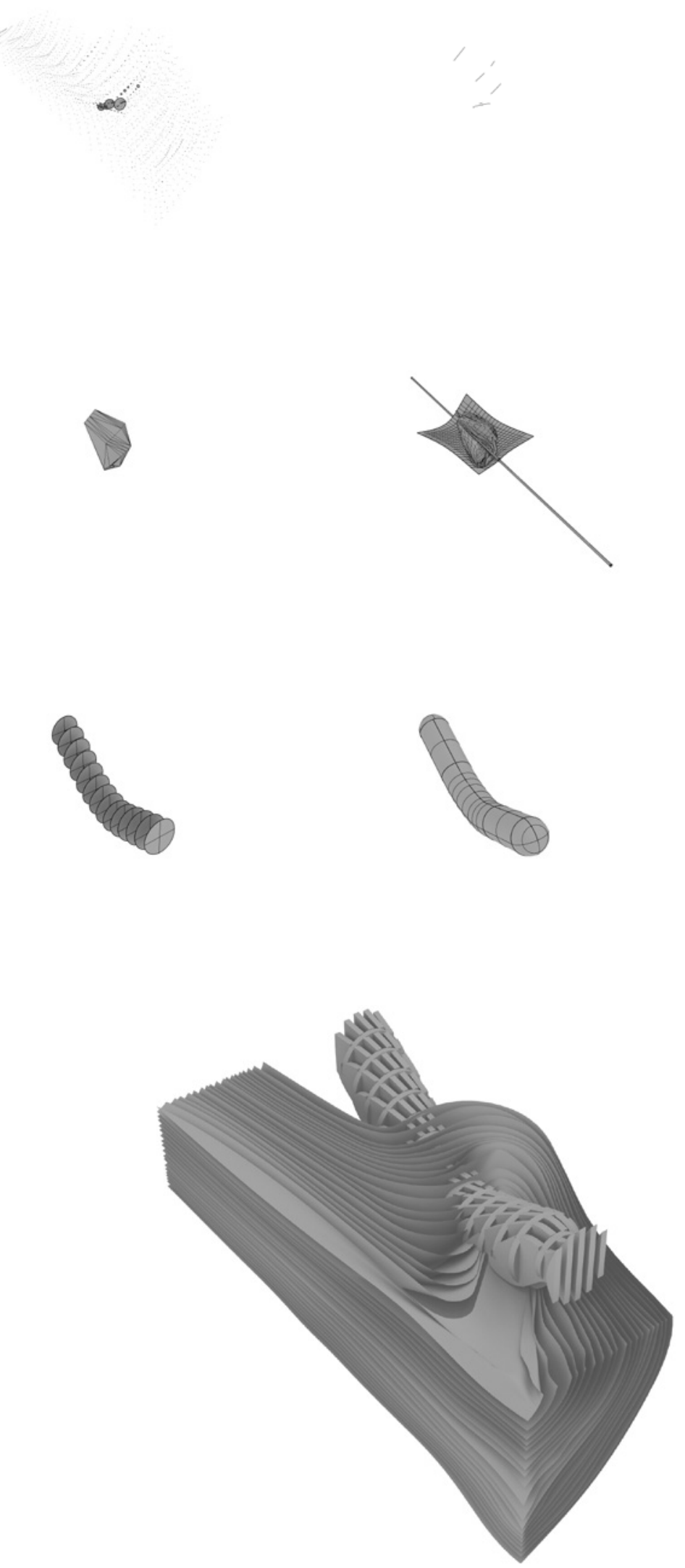

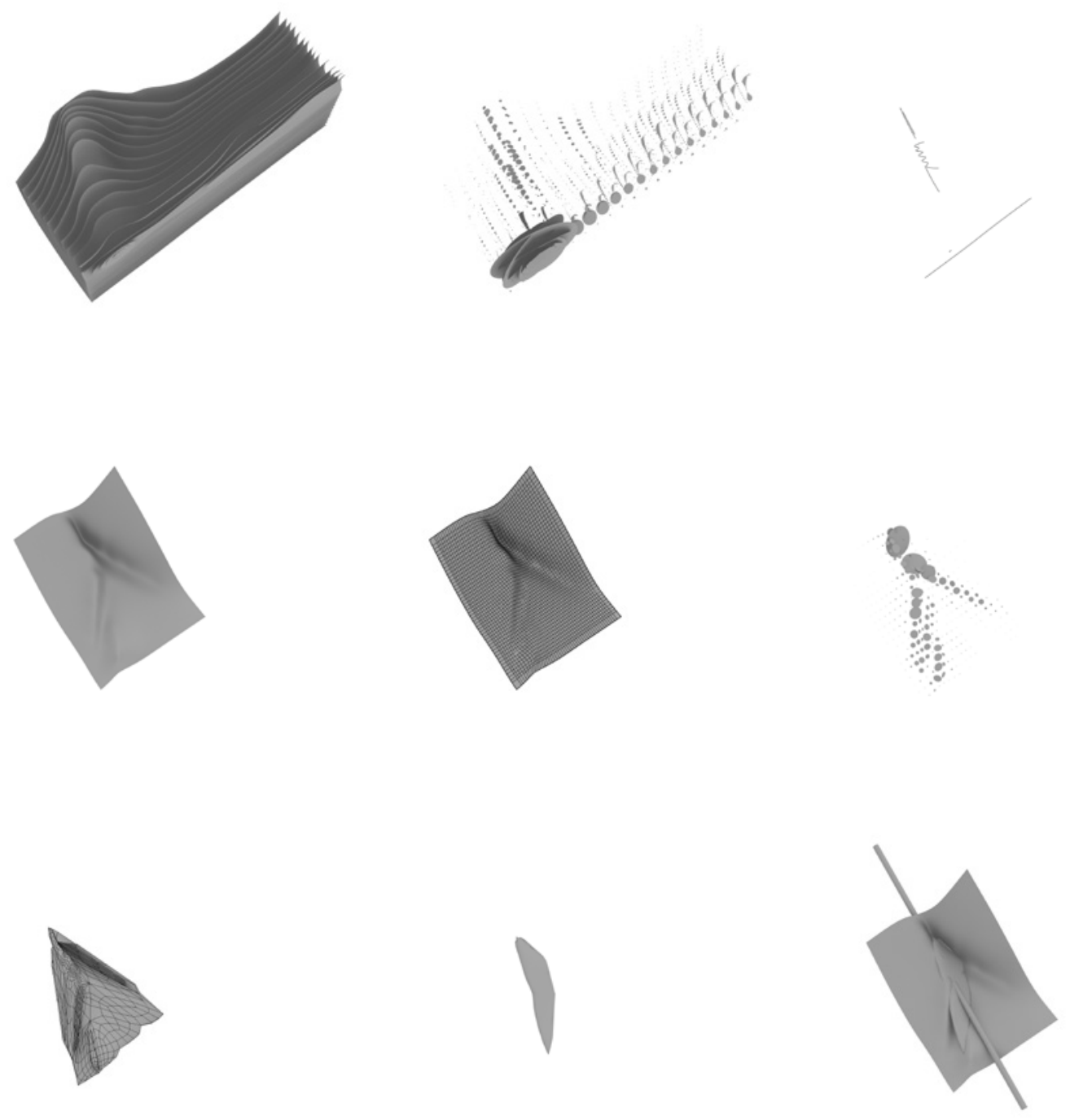

\section{Grafted Branching 3}

Through the iterative process, additional methods became incorporated in the system to investigate other ways of finding the growth phenomena system (shown in the renders above).

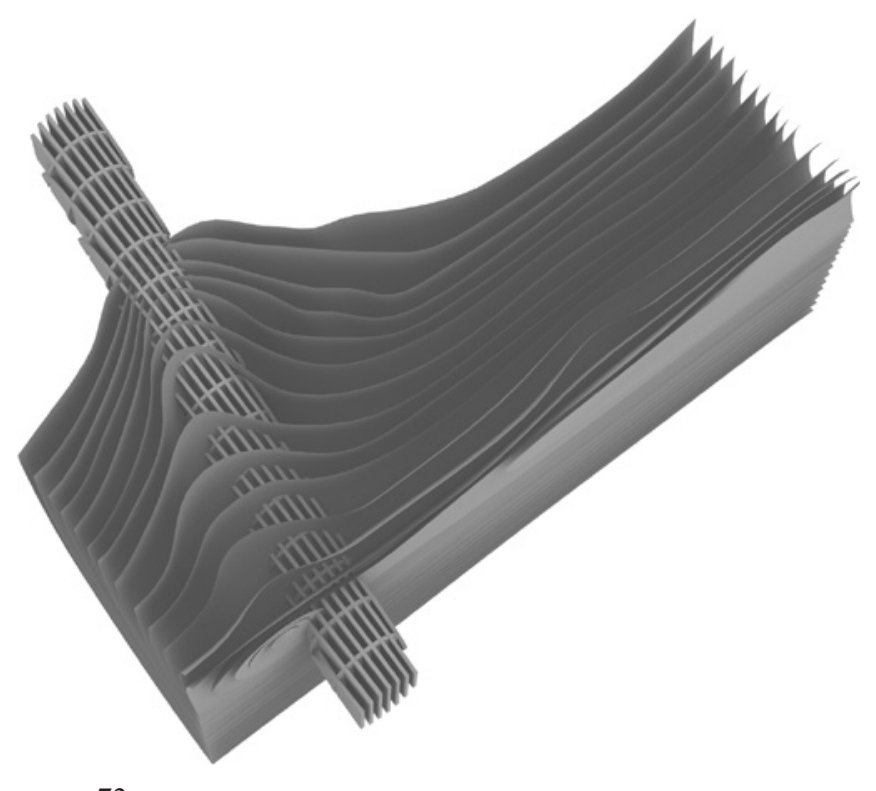


Chapter 3

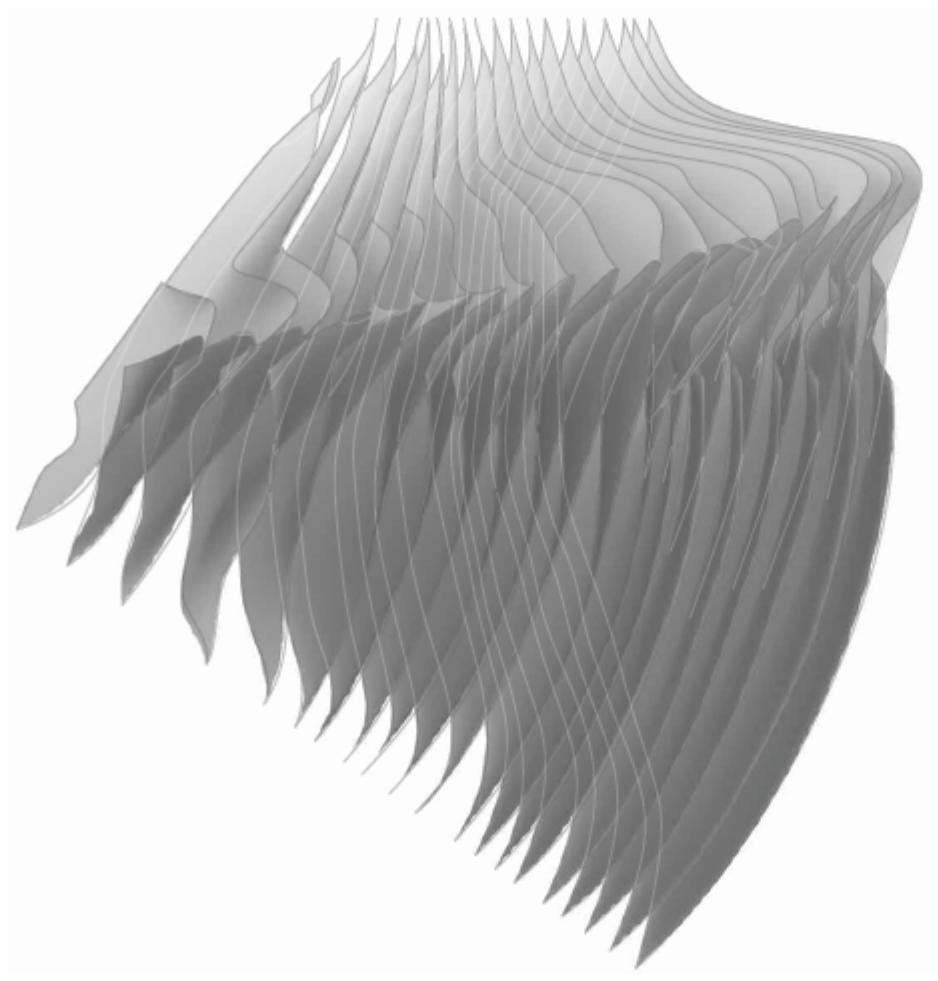

Initial growth ring surfacing

$-73-$ 


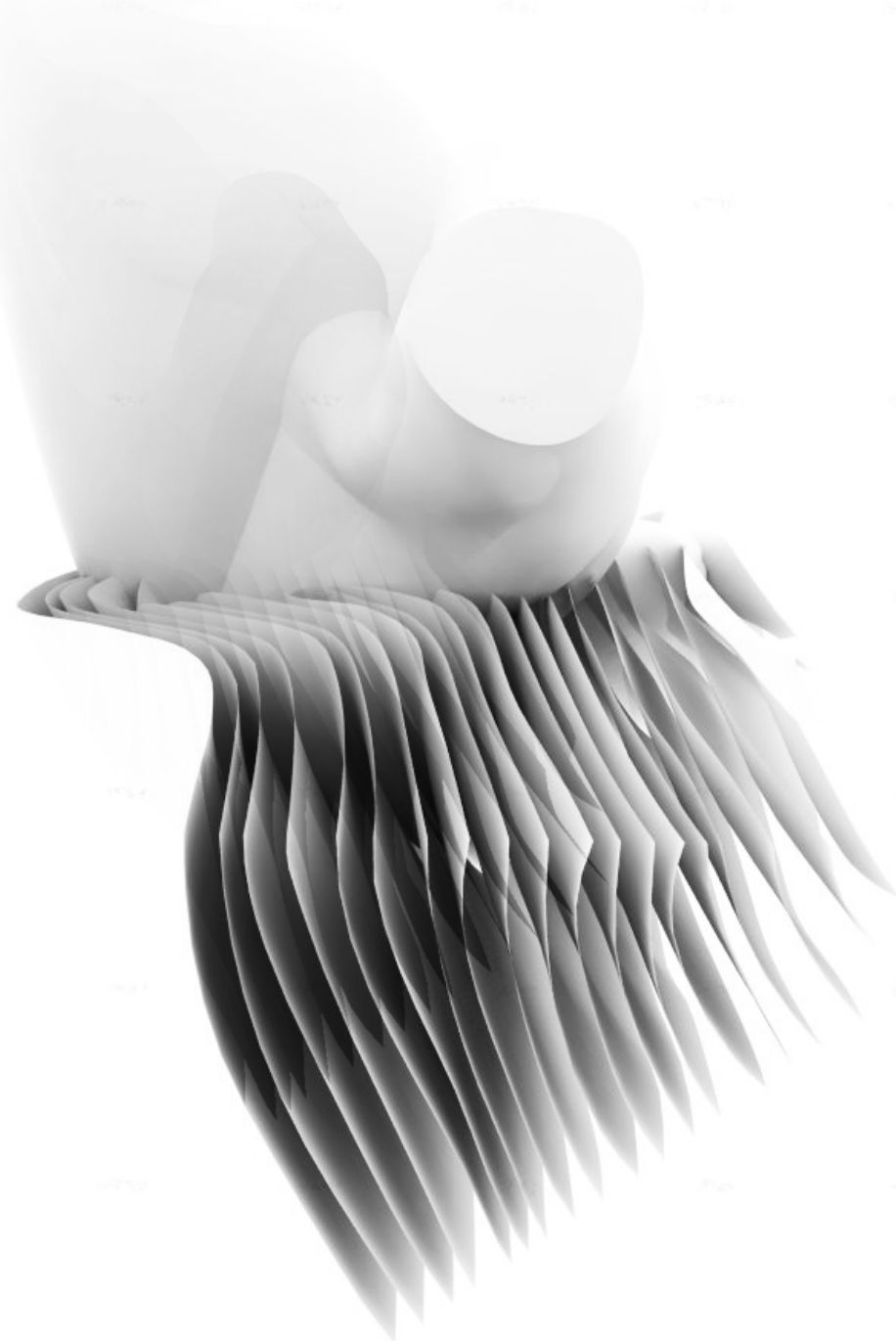



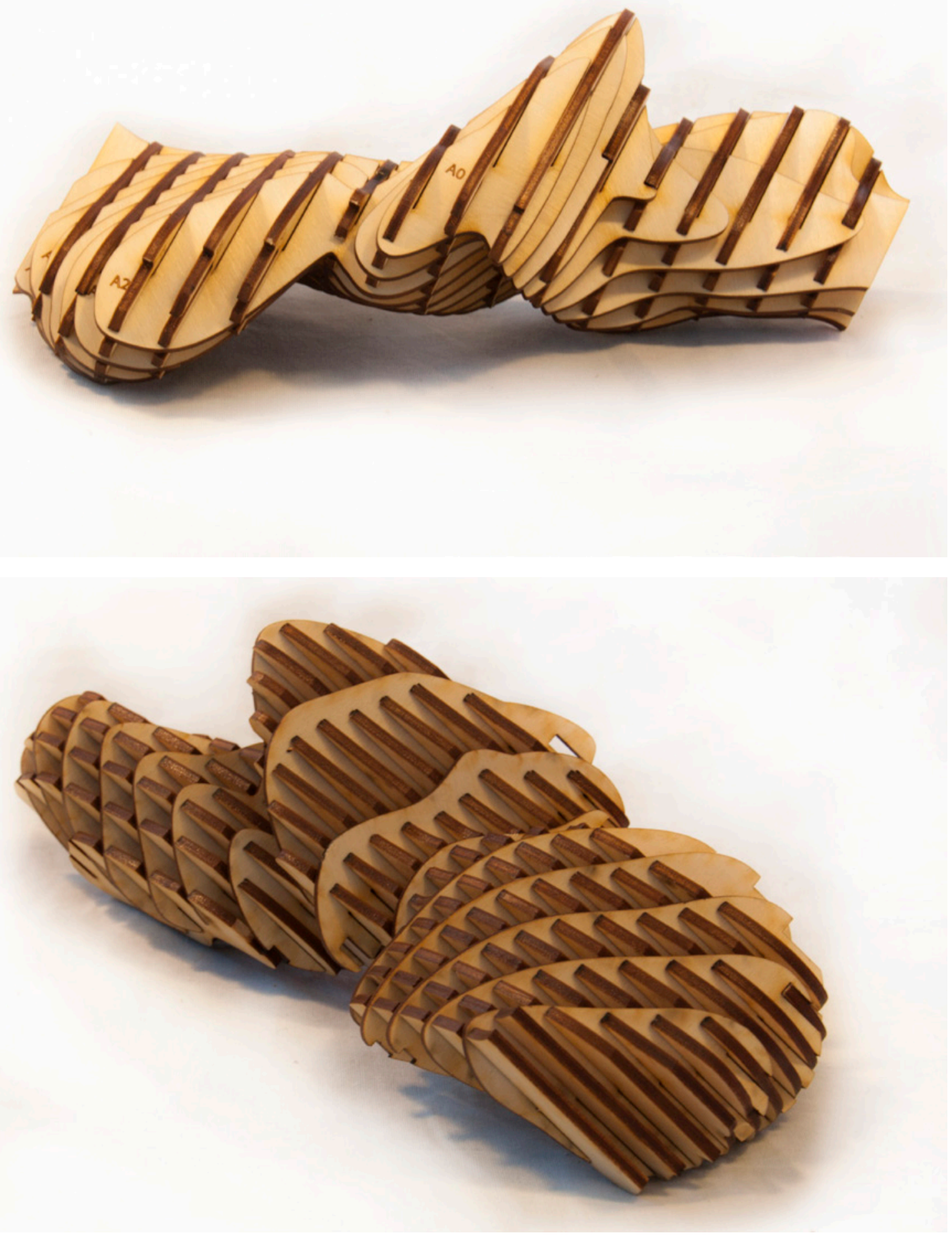

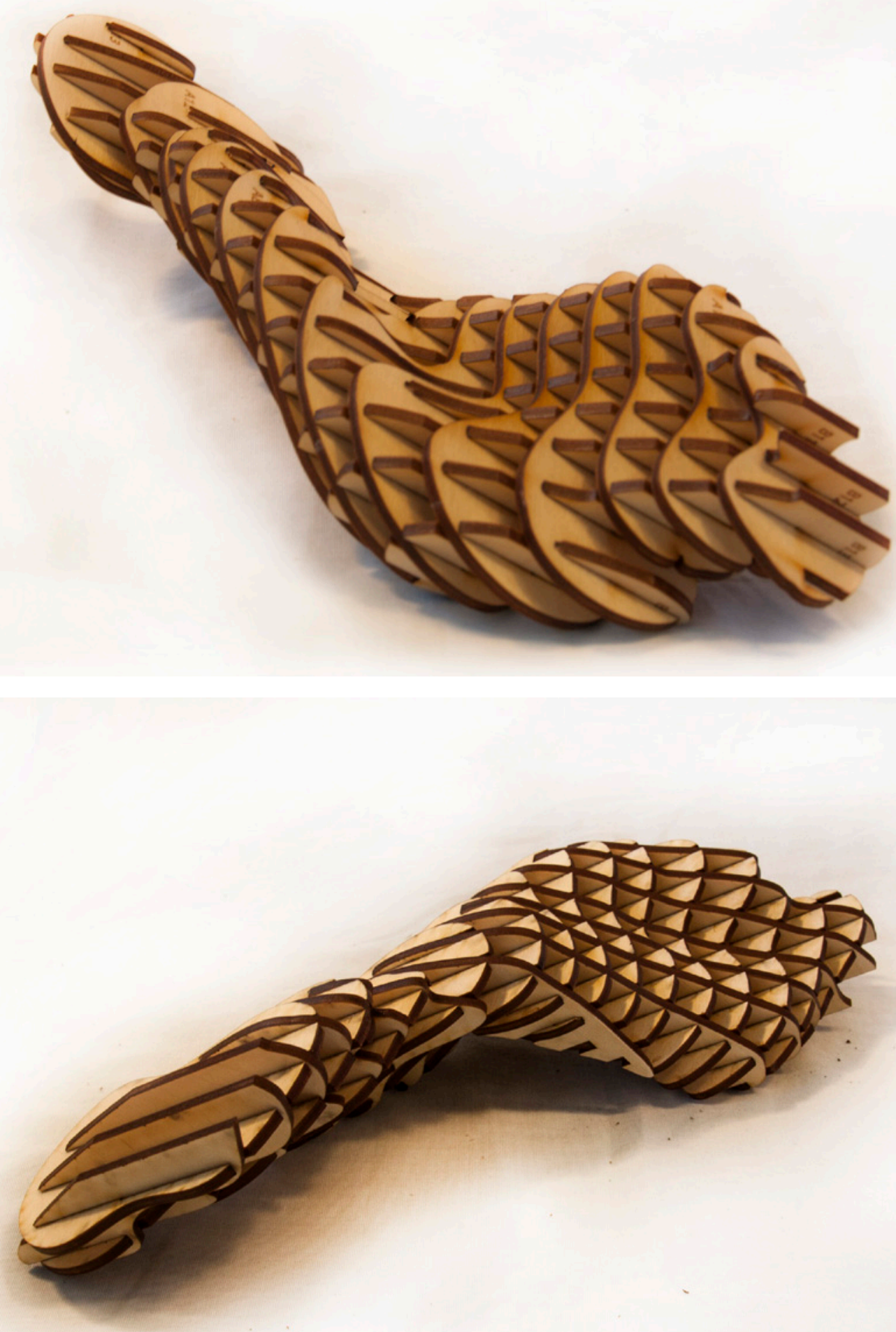
Using a simple contouring method, this ghost growth can be recreated physically and mounted onto the original piece of wood (below).
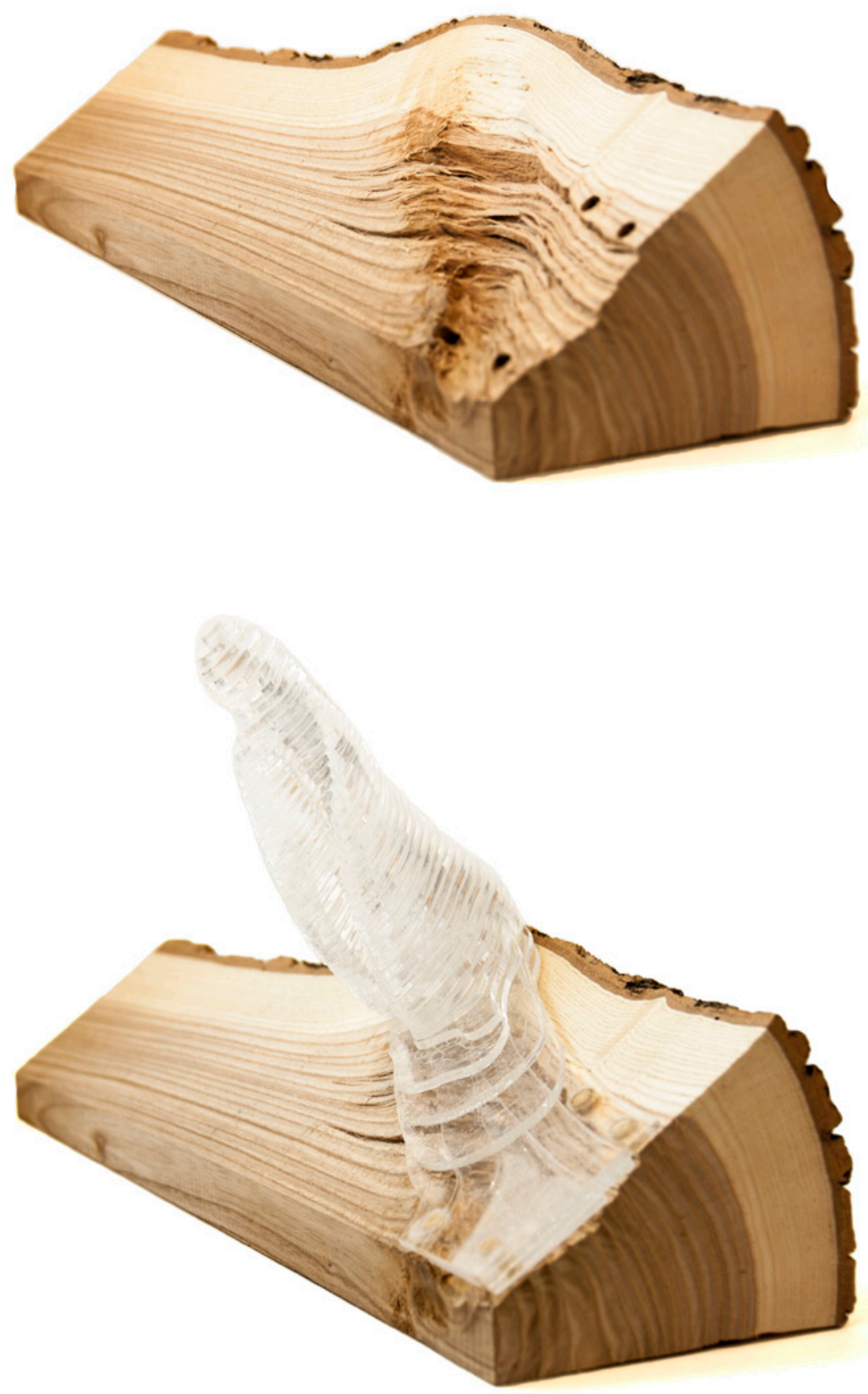


$$
=
$$



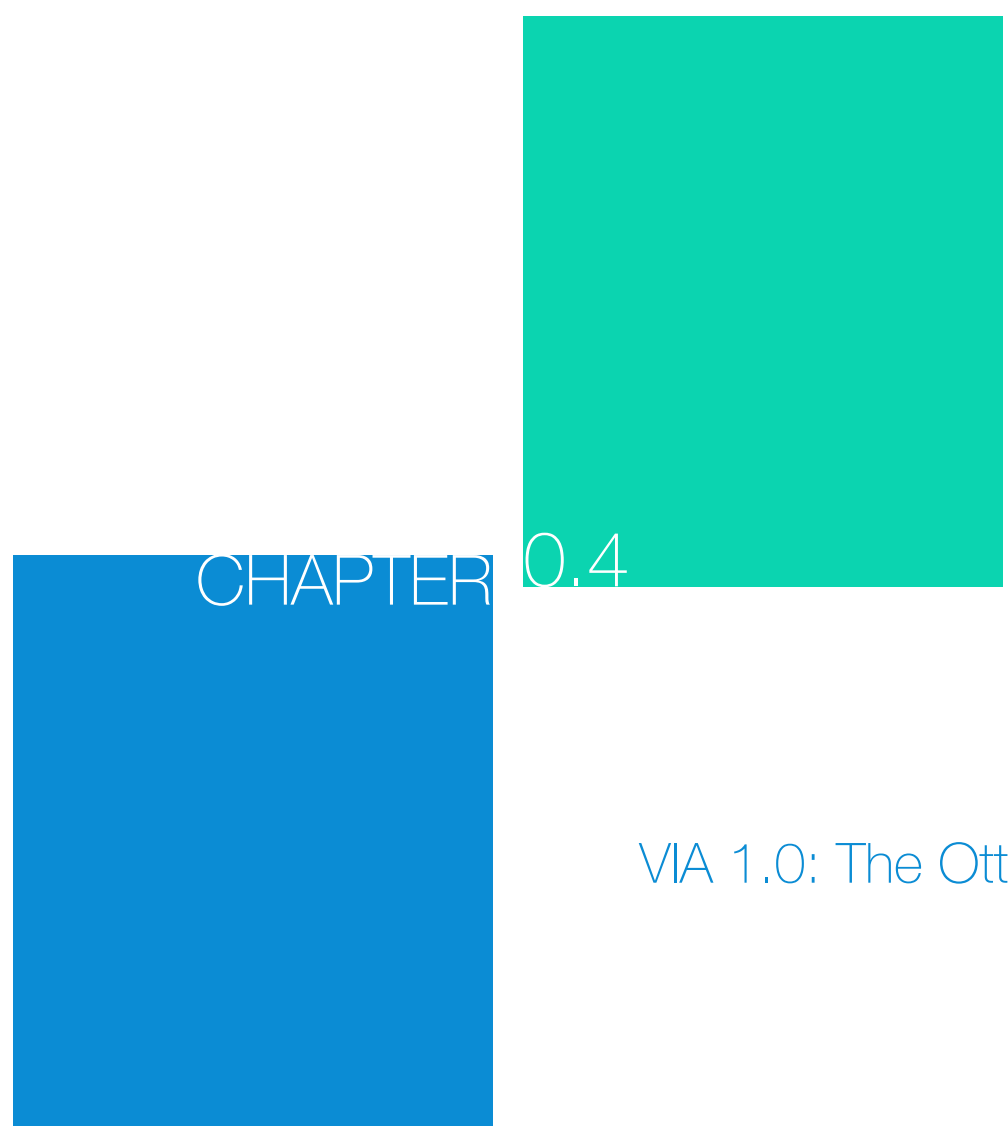

VIA 1.0: The Ottawa Train Station 
"While our international competitors are already at work on high-speed infrastructure, can Canada afford to ignore it?" _ Joseph Soares, The Huffington Post 


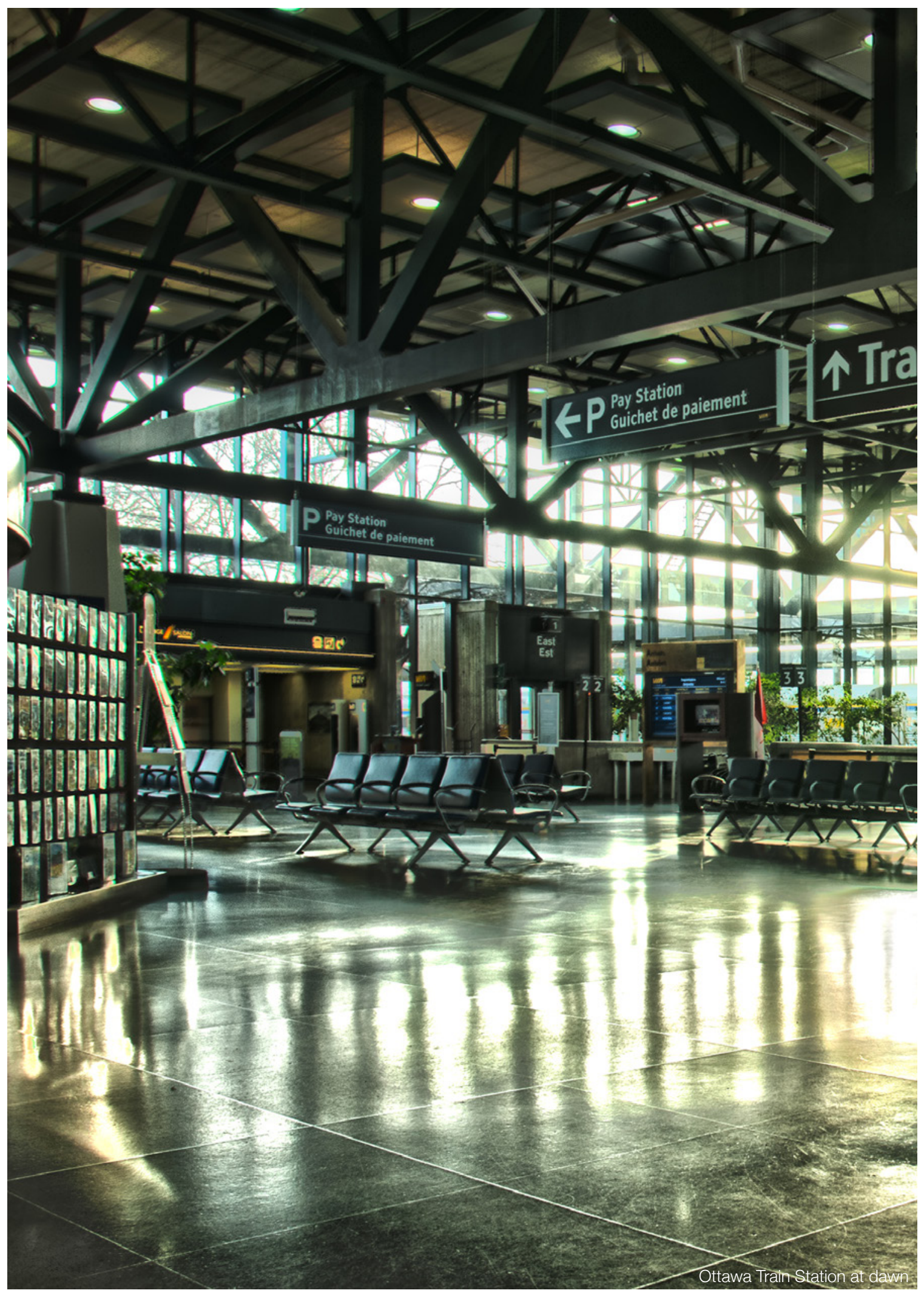




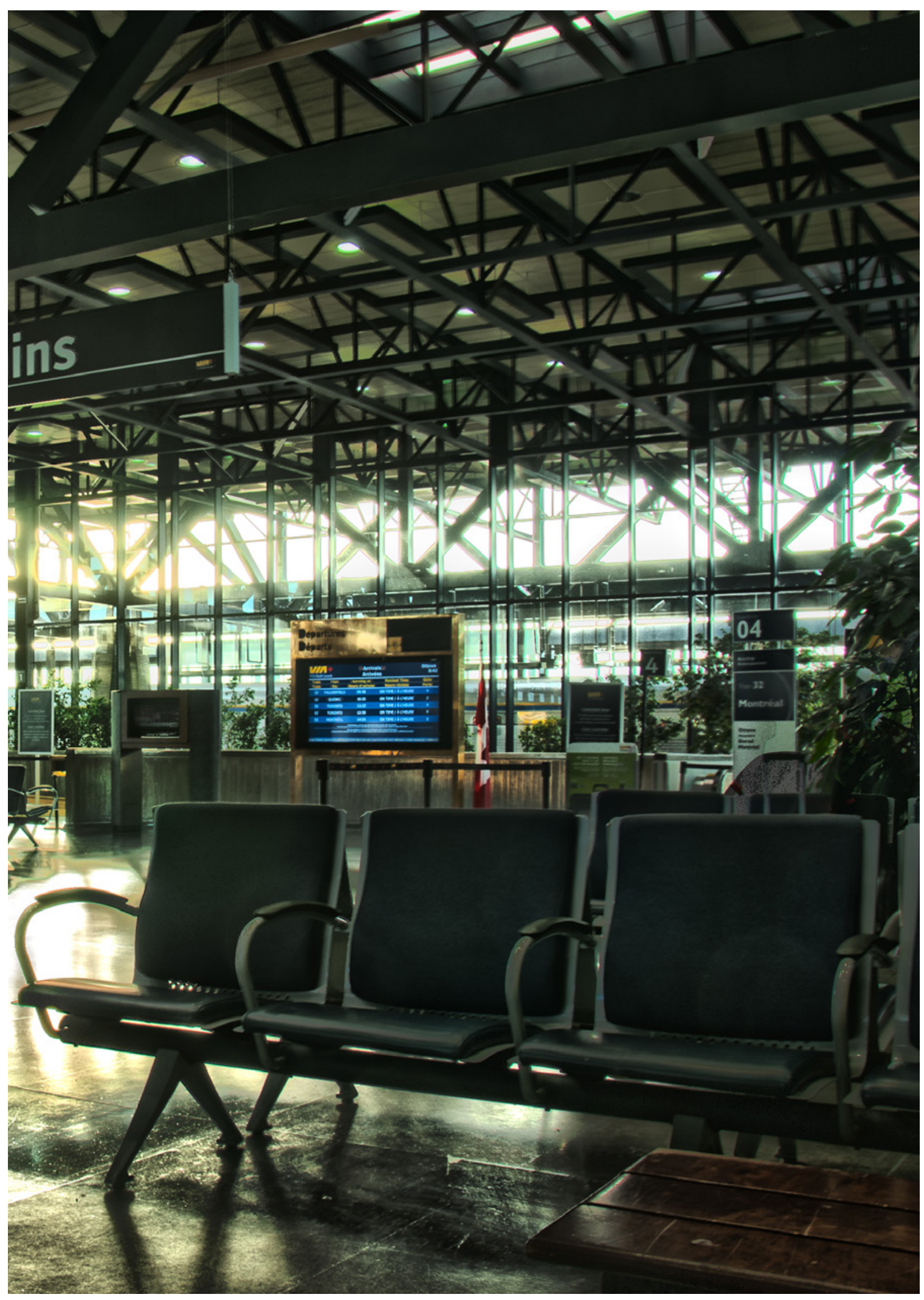



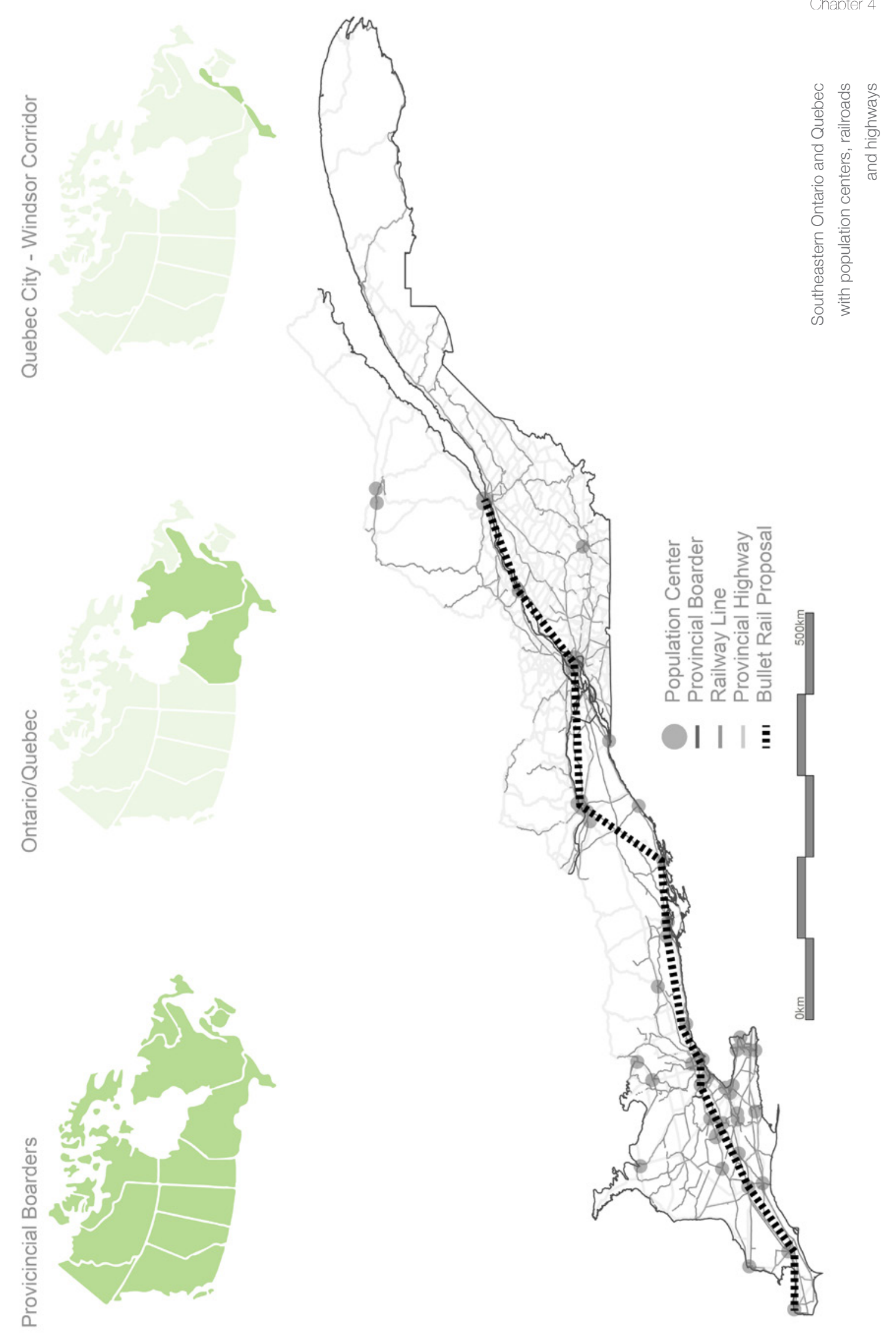

亦峦恋

U 잉 은

은 $\frac{\bar{\sigma}}{0} \frac{\bar{\pi}}{\frac{\pi}{0}} \overline{\frac{\pi}{\pi}}$

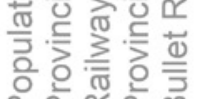

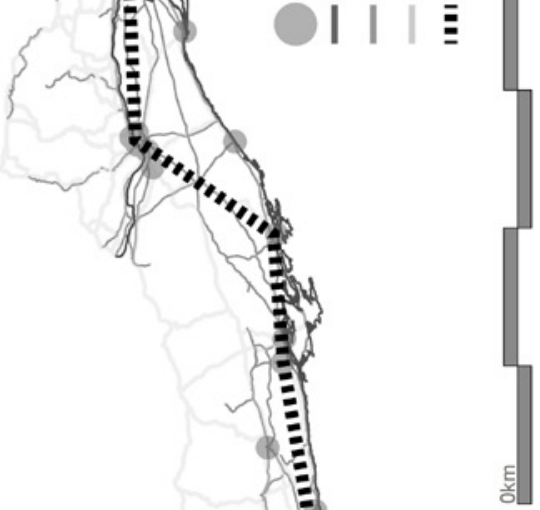




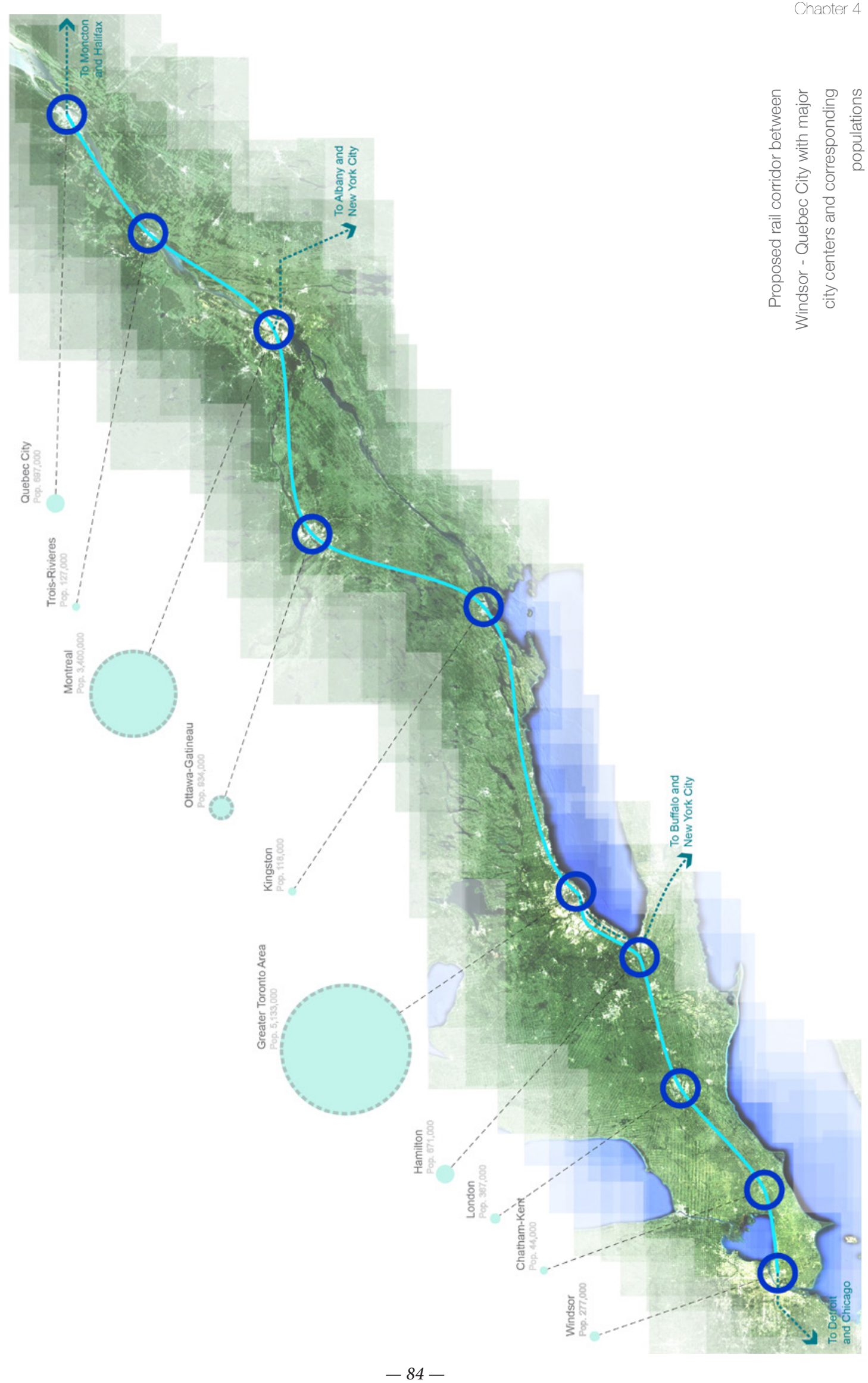



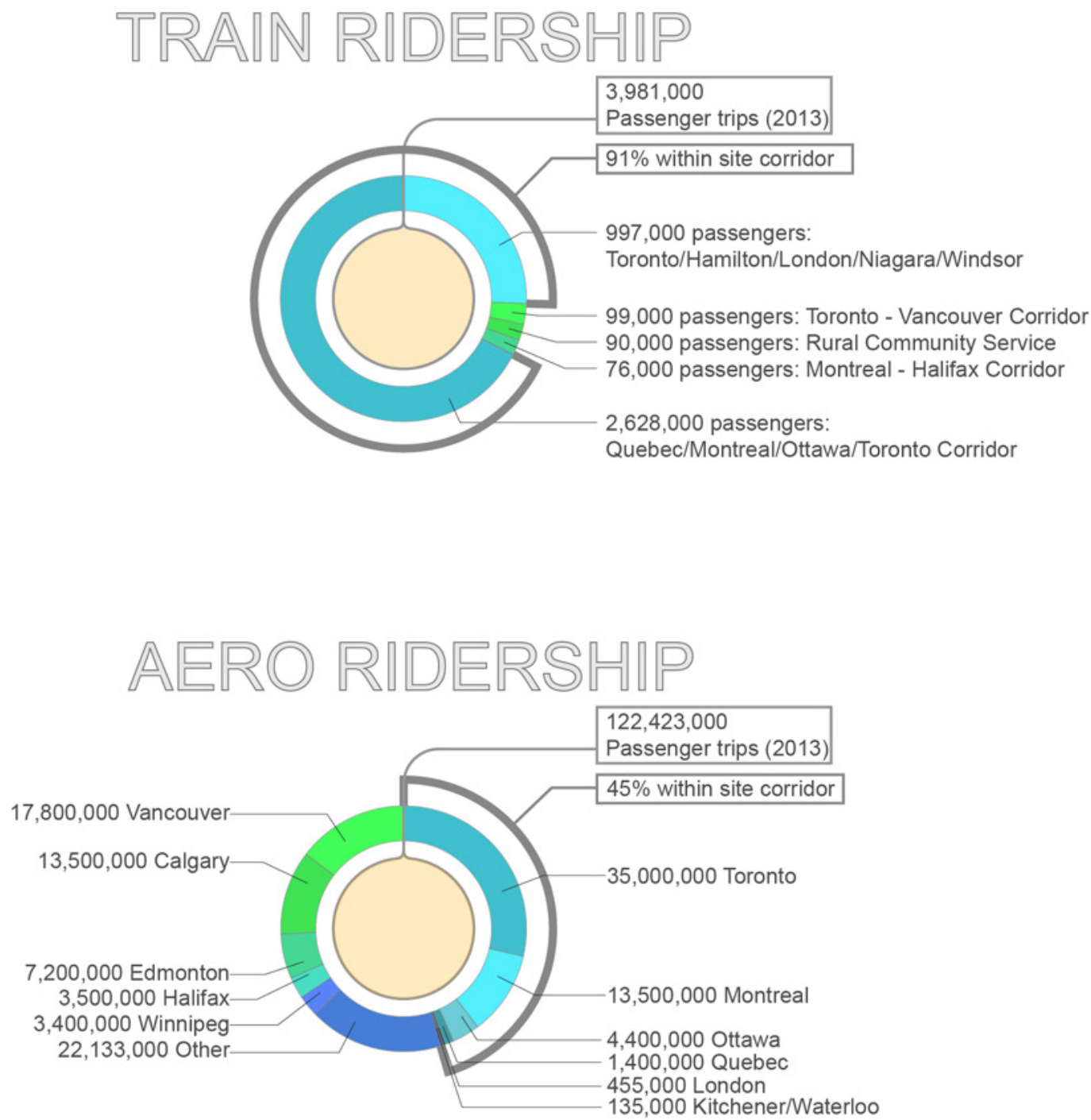
Ottawa-Toronto
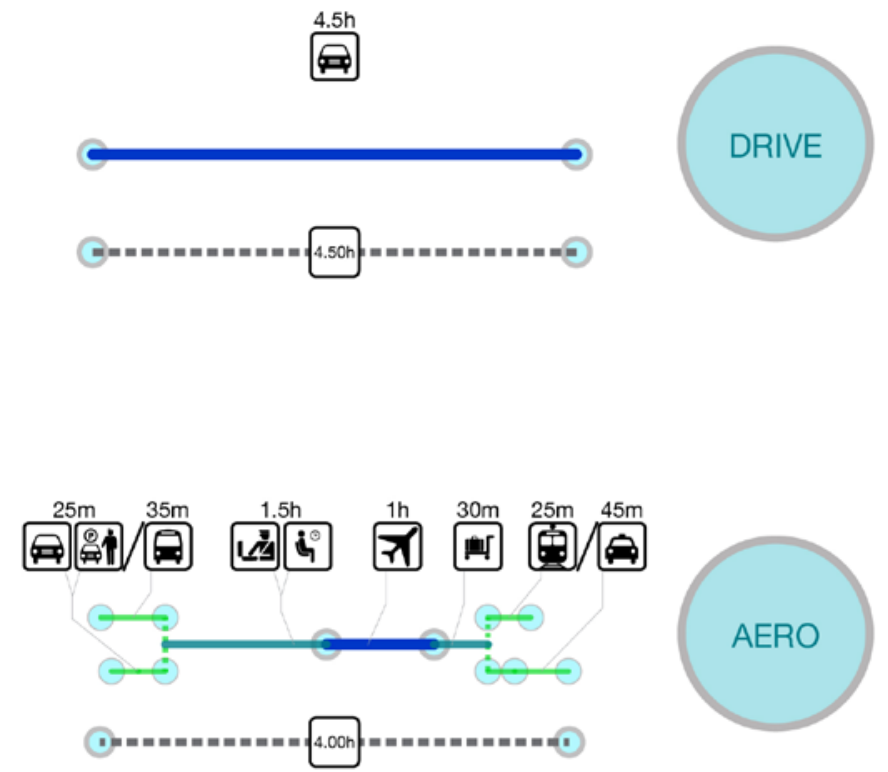

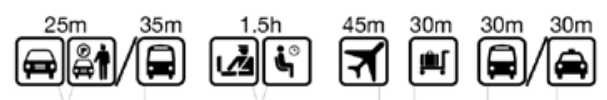
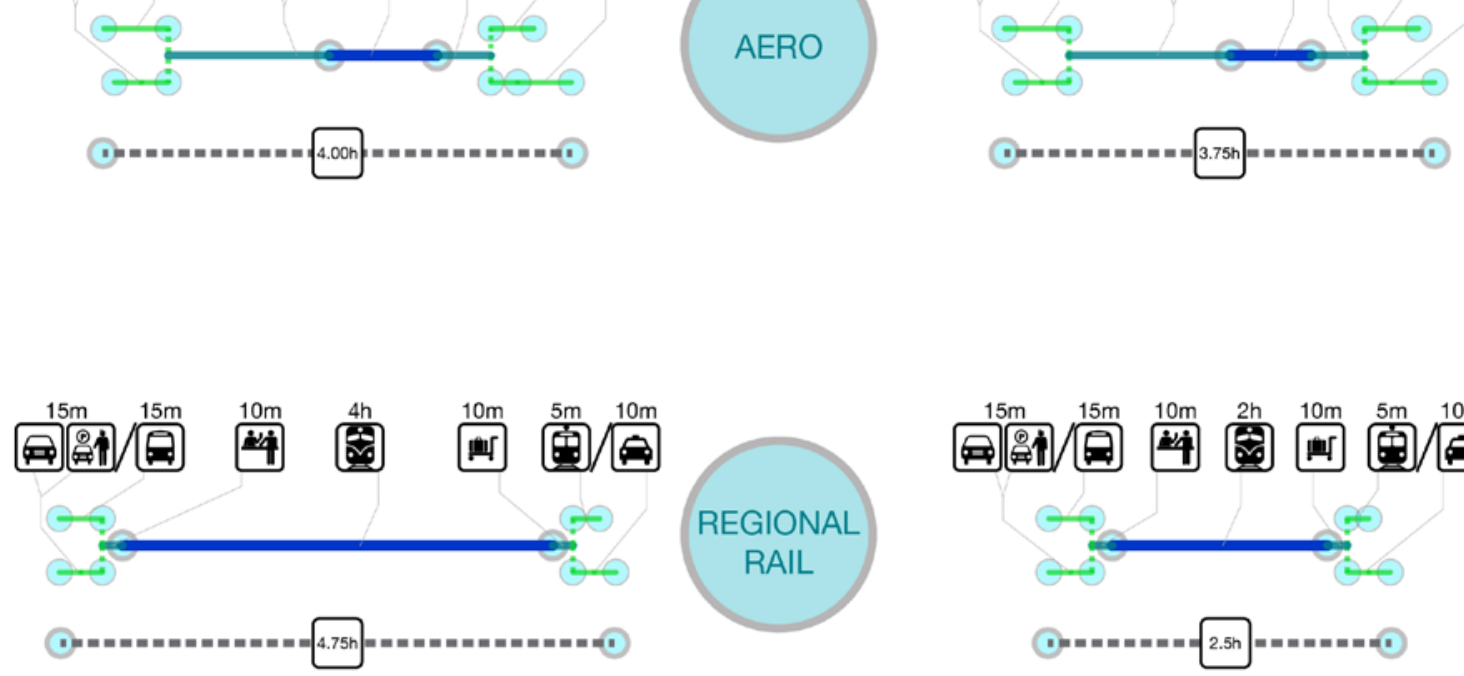

REGIONAL

RAIL

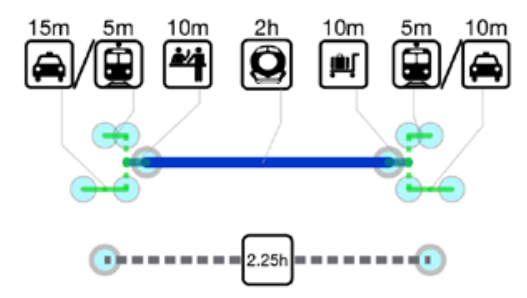

Ottawa-Montreal

占

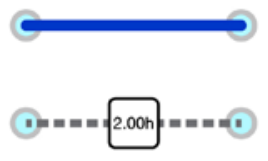

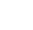




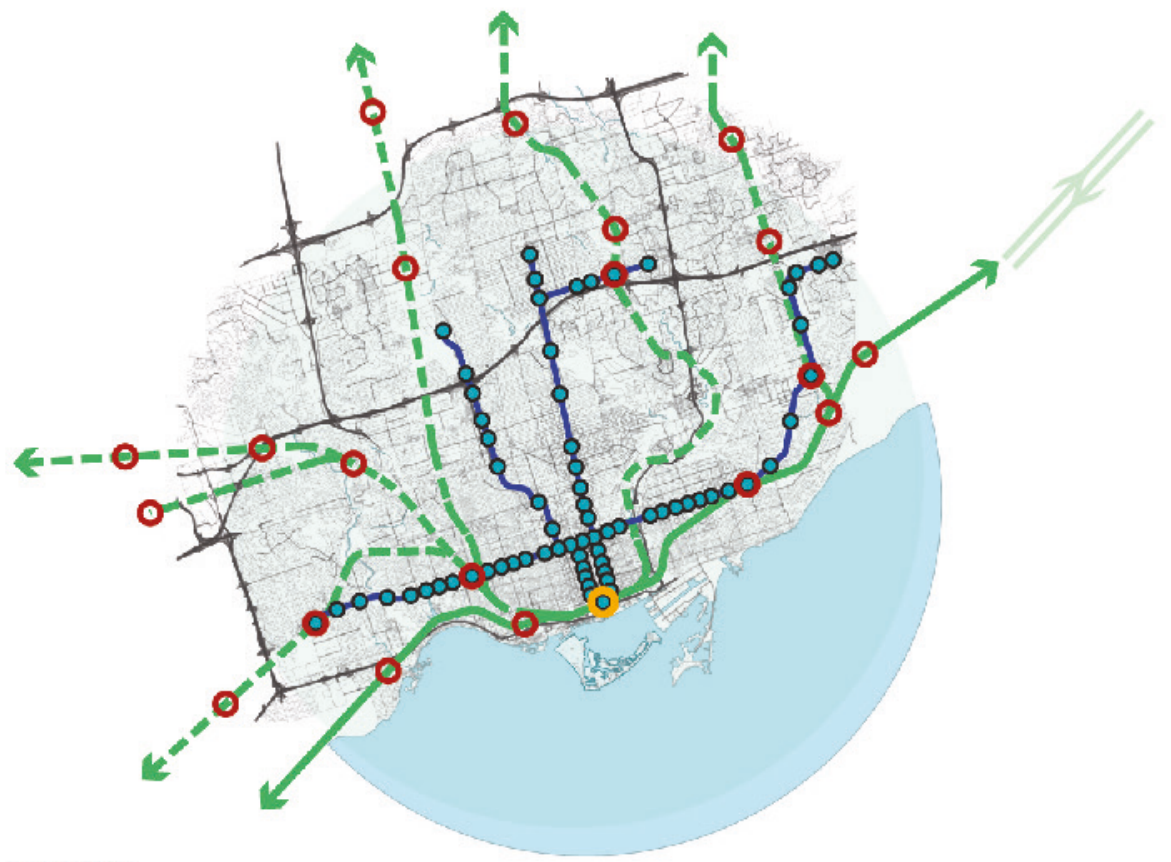

Toronto

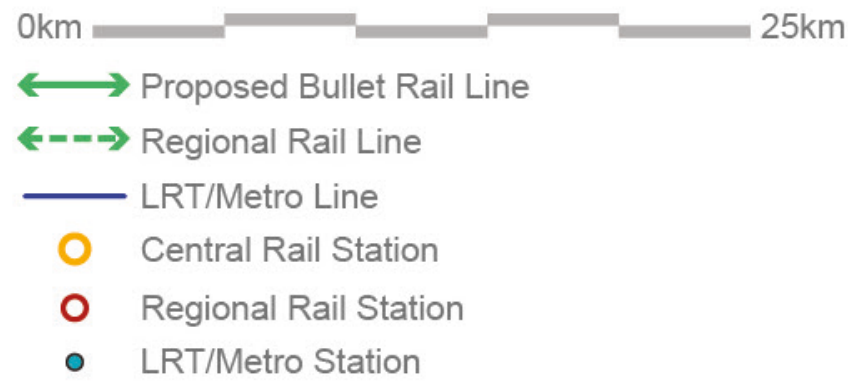




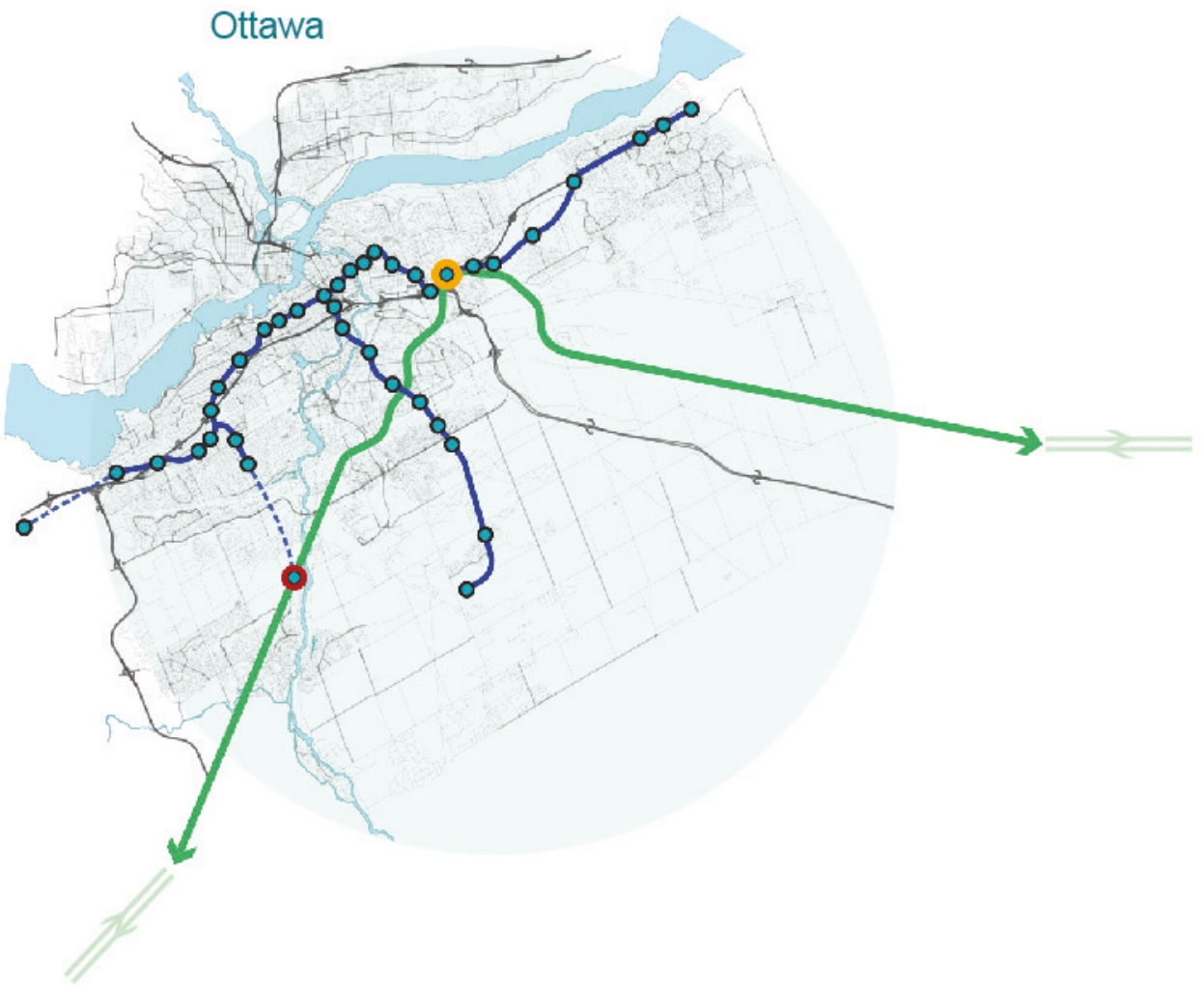




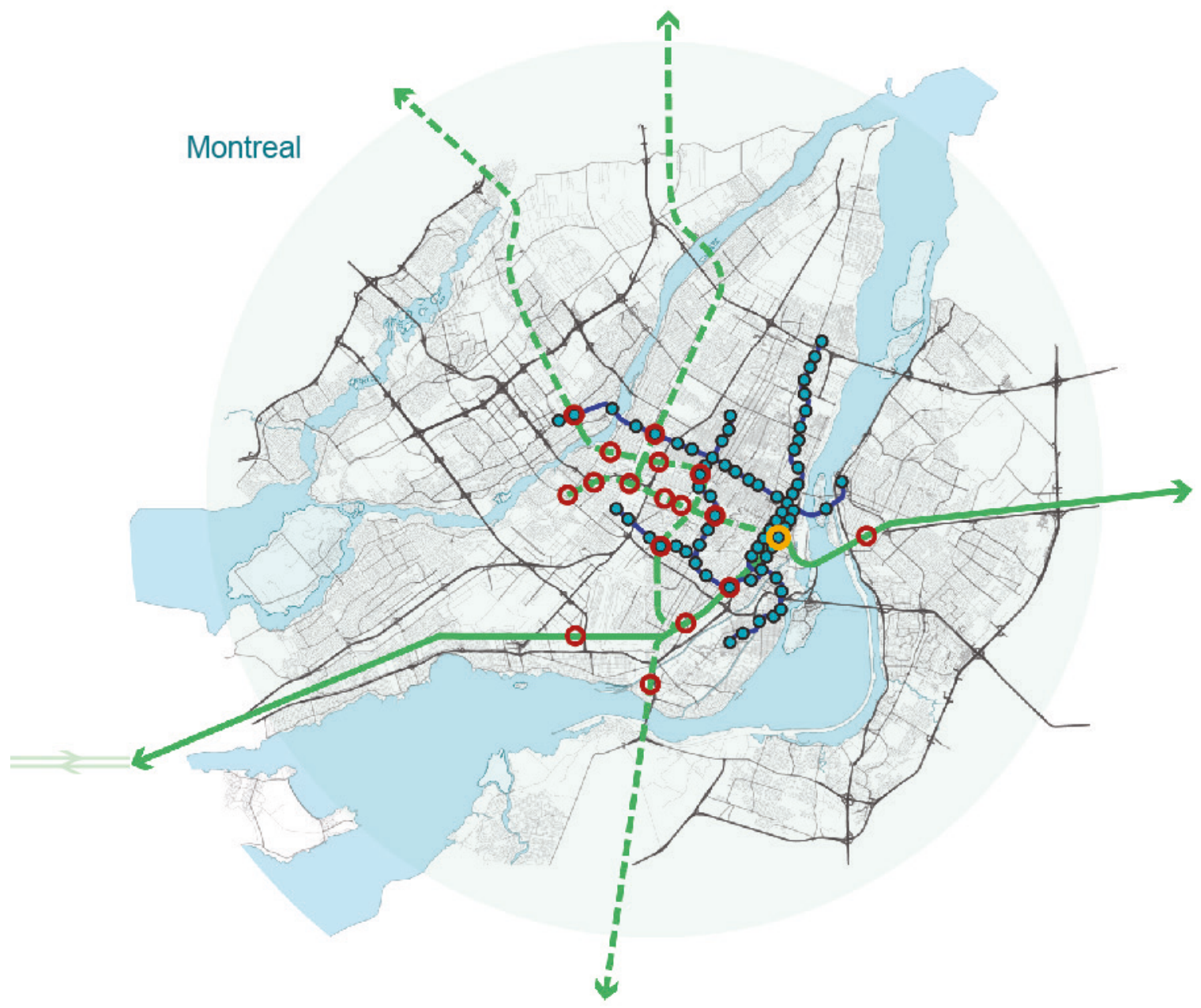




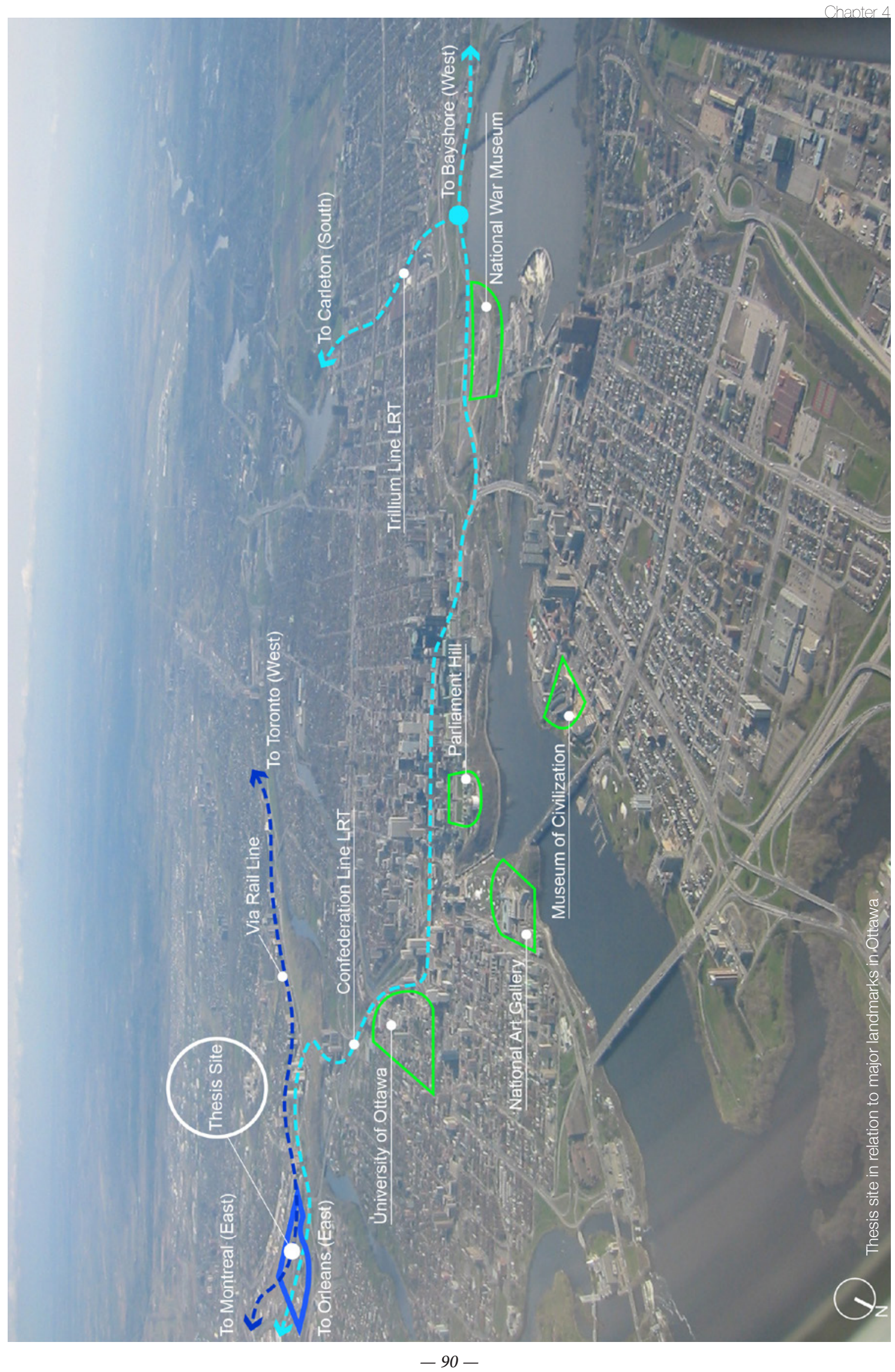




\subsection{HIGH-SPEED FOR OTTAWA}

The Ottawa Train Station was created to consolidate

all train traffic into and out of the National Capital

Region through one single station. ${ }^{1}$ As an aggregator of regional transit, it acts as an iconic entry to the city with the architects and engineers seeking a Modern structural expression to match "the excitement of train travel with an equal bravado of structure. The challenge to span a railway terminus with a bold metaphor of iron or steel in one superbly simple gesture demonstrating the reach of modern engineering technique." 2 John Parkin Architects was chosen for the project with Gene Kinoshita acting as the project architect, completing it in 1966 with a focus on presenting a strong structural expression to bring Ottawa into the Modern era. It was originally named Union

Station because Canadian National Railway and Canadian

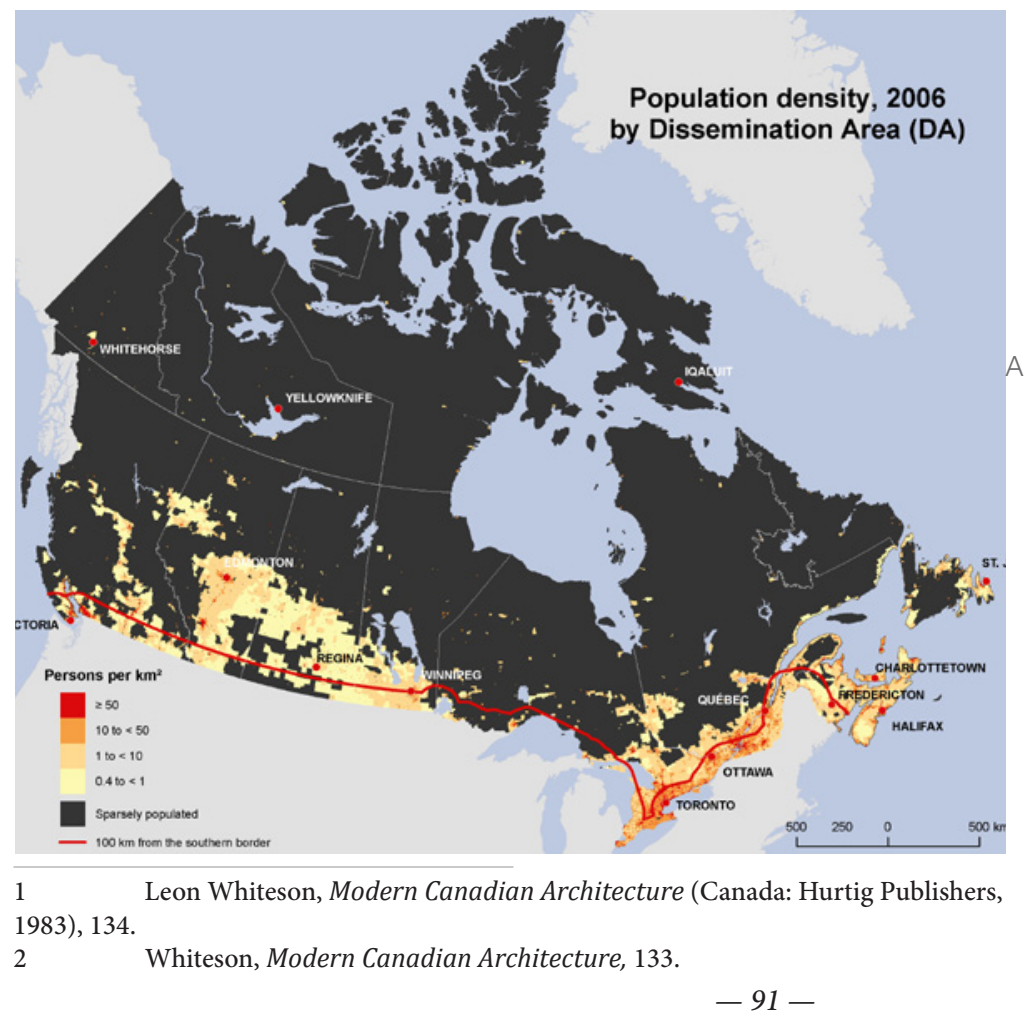


Pacific Rail planned to co-operatively use it as a central

headquarters for both companies. ${ }^{3}$

Unfortunately, as the rest of the world continued to expand their rail systems, Canada has not. It is now the only G8 country without a high speed train line. The definition of a "high-speed" rail line varies minorly across countries, but is generally considered to be one reaching speeds of over $200 \mathrm{~km} / \mathrm{h}$, typically up to $300 \mathrm{~km} / \mathrm{h} .{ }^{4}$

With the new light rail transit (LRT) system moving forward in Ottawa and connecting to the existing train station, this thesis considers the expansion of the Ottawa Train Station to incorporate the LRT with the heavilystudied bullet train system in the Quebec City - Windsor corridor. According to VIA Rail, this line already accounts for approximately $80 \%$ of national ridership. This would amalgamate the three hierarchies of interprovincial, regional and local rapid mass transit into one key node in the city and create a viable, quick, direct mobility option that would improve the connectivity of the estimated

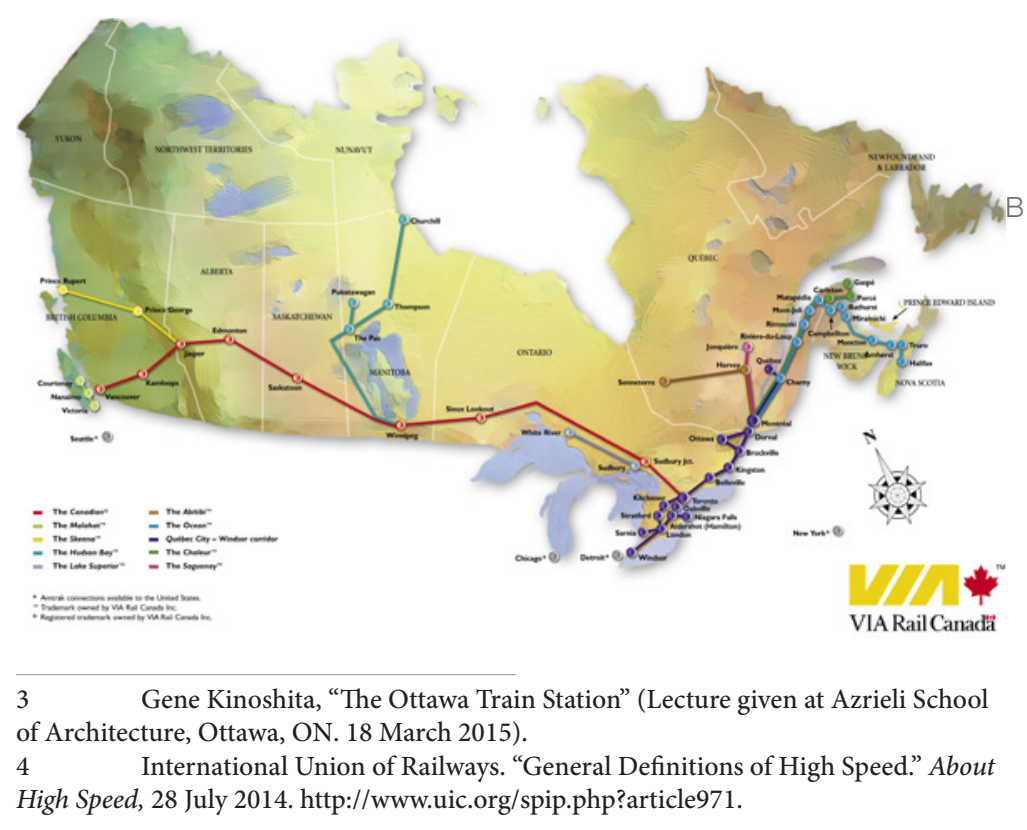


$18,000,000$ Canadians who live in that corridor - over half

the country. ${ }^{5}$ Furthermore, it would plug in to the central subway stations in both Montreal and Toronto, vastly expanding the feasibility of door-to-door movement for Canadians through this dense urban corridor.

The advantage of the Ottawa Train Station expanding to service as a premiere method of travel for this urban corridor lies in the simple, full-service, doorto-door movement it can provide. Even though actual in-air transportation time is shorter, aero travel requires significant additional time allowances when compared to rail. Airports are typically removed from the urban hub for noise reasons (especially in Toronto, Ottawa and Montreal), meaning more transit time to the departing airport. Expanded airport security means passengers are required to arrive 60-90 minutes prior to boarding. Baggage check and reception are carried out at a significantly slower rate. By contrast, central rail terminals are typically located downtown, include direct rapid transit, and have no preboard ticketing, security or baggage.

Factoring for all of these differentials, actual doorto-door transit times start to even out across short- and mid-range distances, particularly for the context of the Windsor - Quebec City corridor. This does not dismiss aero transport, but it means that its full advantage is pushed towards servicing longer distances or ones that have indirect or non-existent land connections. With an expedited high-speed rail line, a bullet train would offer

5 Central Intelligence Agency, The World Factbook: Canada. https://www.cia. gov/ library/publications/the-world-factbook/geos/ca.html. 
a simplified, streamlined service that would effectively

replace many aero trips along the route.

\subsection{NODES AND NODE-BASED SYSTEMS}

Kevin Lynch's Image of the City provides a detailed analytical review of the main elements that make up the city environment, where visual cues provide an elemental method of placing a physical being in a space. "These clues for urban design can be summarized... since there are common themes that run through the whole set," ${ }^{6}$ with the five distinctions being a) Path, b) Edge, c) District, d)

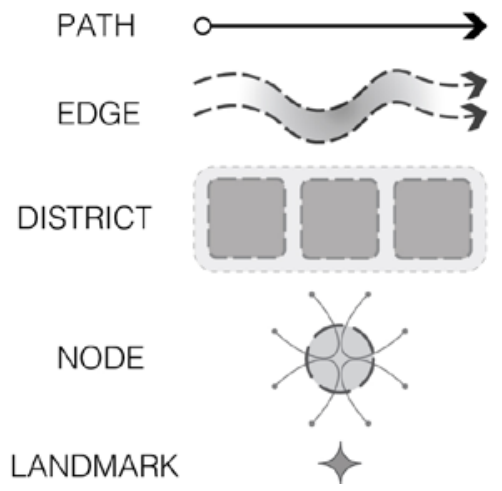
Node, e) Landmark. The importance of these five elements is constituted by their ability to help man orient himself in space $^{7}$ and therefore define a distinct 'place' in the mind of the user. This 'place' is created by the overlapping elements Lynch describes, which reinforce a hierarchy of distinction that the individuals can perceive for identification between themselves and specific objects.

More broadly, Stan Allen describes similar urban make-ups as 'fields', denoting their omnipresent relationship to one another as pieces within a whole. This worldview promotes "a practice not devoted to the production of autonomous objects, but rather to the production of directed fields in which program, event, and activity can play themselves out." 8 The architecture 
reinforces the functional condition of the program without

defaulting to Functionalist tropes.

Paths, Edges and Districts give spatial clarity by separating space to varying degrees, whether through physical or implied barriers. These denote the effect of direct traffic movement on a space, while establishing the relationship between served and servant spaces without constraining them. Most importantly, their direction acts as a means to connect to surrounding spaces through their role as primary vectors for movement. Populous outlines this by describing an ideal path network as one that resembles a tree's structural hierarchy. In this case, "the individual twigs lead to small branches, then to larger branches, and ultimately to the trunk." ${ }^{\prime 9}$ This reduces the likelihood of a counter-traffic flow, which can cause confusion and congestion in crowd movement. They instead suggest an ideal for providing spectators with "a series of simple $\mathrm{Y}$ or $\mathrm{T}$ junctions as they move"10 so that there is only one decision to make at any time in wayfinding.

In more general terms, "when Walter Benjamin writes that 'construction fulfills the role of the unconscious,' he articulates the capacity of certain structures to act as a scaffold for a complex series of events not anticipated by the architect - meanings and affects existing outside of the control of a single author that continuously evolve over time." ${ }^{11}$ By defining the relationship rather than the object,

9 Geraint John et al., Stadia (New York: Routledge, 2013), 175.

10 John, Stadia, 171.

11 Allen, Points \& Lines, 54. 
the architect doesn't need to anticipate the minutiae of

how the structure will inform the event. Rather, the event works inside a given solution space that the relationships can reinforce, with enough flexibility to accommodate for unaccounted surges.

Alternatively, "junction nodes occur automatically at major intersections and termini, and by their form should reinforce those critical moments in a journey." ${ }^{12}$ The node-based systems therefore establish the direct spatial implications as anchor points from one to the next. This is because of their natural creation due to being points of high change/use/traffic, etc. With a digital methodology, these relationships can be extracted and redefined, because of their shared characteristics to flow networks as paths, edges and districts. The node-based systems localize a network between a series of points based on their direct relationships. This, as Woodbury clarifies, addresses the problem directly: "Rather than the designer creating the design solution [by direct additive/subtractive manipulation] the idea is that the designer establishes the relationships by which parts connect,"13 allowing the designer to avoid excessive personal tropes that might be influencing biased decisions and instill integrity into the working methodology. In this way the designer will focus on how to "express and explore relationships... and [an] entire parametrically described solution space" 14 by removing the control of the singular, autonomous author

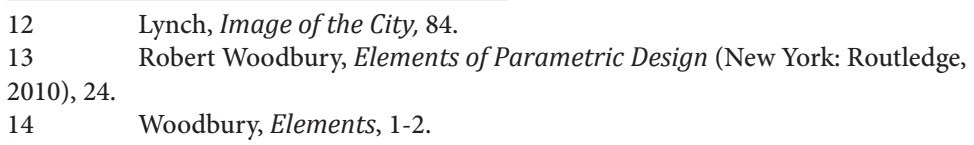


described by Benjamin.

Furthermore, Woodbury points out that

because the parametric process ignores the additive/ subtractive method of modeling in favor of one defined by relationships, the node-based methodology becomes inherently scalable. The growth and movement can update and react to its own evolution as more information becomes clearer, and "although ratios can be expressed numerically, the relationships intended are fundamentally geometric." ${ }^{15}$ The one drawback that arises from this is how a scalable model can also be interpreted as scaleless if there is no base reference point. In the same way that a mathematical function needs an input to define an output, so too does the solution space: "Field configurations are inherently expandable; the possibility of incremental growth is anticipated in the mathematical relations if the parts." ${ }^{16}$ If there is no base scale for reference, one must be incorporated into the model to ground the work. The purity established by the parametric safeguard suggests a similarity to the patterns found in natural phenomena due to their shared lack of bias. Both exist in a vacuum - one digital and one natural. There are external influences on their shape and growth patterns, but these constitute internal biological reactions to external stimuli and are important parts of the system - they reinforce the neutrality. Inside these growth phenomena, the external factors that influence the form are much more

15 Stan Allen, Points \& Lines (New York: Princeton Architectural Press, 1999), 
important than the form itself, since "overall shape and

extent are highly fluid and less important than the internal relationships of parts, which determine the behavior of the field." ${ }^{17}$ As an internal system of disparate parts influenced by external forces, the patterns can be tracked and analyzed through dissection, digital reconstruction, and generative simulation.

Similar to the individual natural nodes that dictate growth pattern phenomena in the space between them, "although static in and of themselves, infrastructures organize and manage complex system of flow, movement, and exchange. Not only do they provide a network of pathways, they also work through system of locks, gates, and valves - a series of checks that control and regulate flow." 18

If one can accept a similarity in the way each system acts, then in this way node-based natural phenomena might have the potential to inform node-based architectural projects. This could be especially useful as a model for projects incorporating fields of continuous physical movement and complex traffic patterns. It therefore stands to reason that within erosive phenomena and natural growth movement lies a latent similarity that can be extracted and applied to architectural applications. Inside both, "there are effects of scale (a capillary effect when the elements get very numerous and very small), both of which tend to produce field conditions that disrupt the overall tendency of [these systems] to organize 
themselves in linear fashion," ${ }^{19}$ producing divergent

relationships across the field. Allen further points out explicitly that since these "infrastructural systems work like artificial ecologies," ${ }^{20}$ their field relationships can be used to inform and dictate the built environment, both at the architectural and urban scale.

"Field conditions are bottom-up phenomena, defined not by overarching geometrical schemas but by intricate local connections." ${ }^{21}$ In the same way that Lynch describes a series of disparate elements (paths, edges, nodes, etc.) interacting to develop a reading of the image of the city, so too does Allen's serve as a model for the relationship between the disparate parts. In a more literal sense, when the seed of a tree is planted, it generates a system of growth based on factors such as water, sun, and nutrients to grow. It is affected by those things in the physical as a biological reaction to the phenomena around it. In the digital those don't exist. Instead, a growth is affected by the inputted 'fields' of surrounding phenomena as Allen and Lynch describe them.

As it stands, four of Lynch's five elements (Path, Edge, District, Node) can be attributed to the current Ottawa Train Station site via recreated phenomena in the digital process - but the idea of a Landmark is so far nonexistent. The pure programmatic consequences of the building act in accordance to the landmark description by Lynch, since by its own admission it is an indispensable

\begin{tabular}{ll}
\hline 19 & Ibid. \\
20 & Allen, 56. \\
21 & Allen, 92.
\end{tabular}


function for any major city, particular a national capital.

That being said, the inherent program is the main driver of it being a Landmark, not the actual building. This project will therefore posit a new landmark for the Ottawa station, as a node-based system that draws from the localized phenomena of growth and destruction in the key elemental building material of historical significance to the region, wood. Ottawa is uniquely located geographically as the catalyst for a new Central Station to incorporate a bullettrain system into the national railway services. As the political center of the country it is ideally positioned to support this kind of proposal. Ottawa deserves a newly expanded train station to become a world-class capital city. 


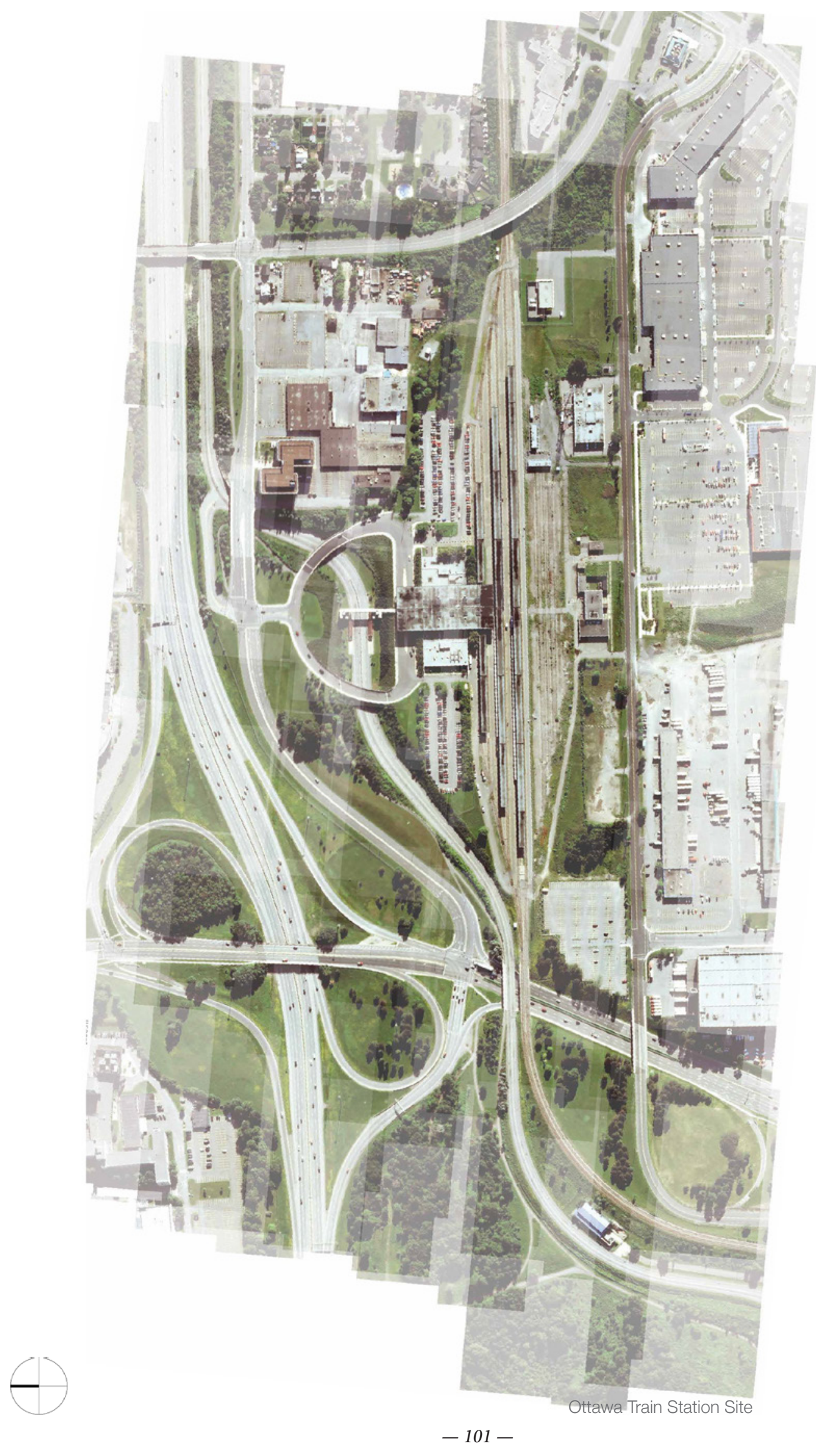

Chanter 4 


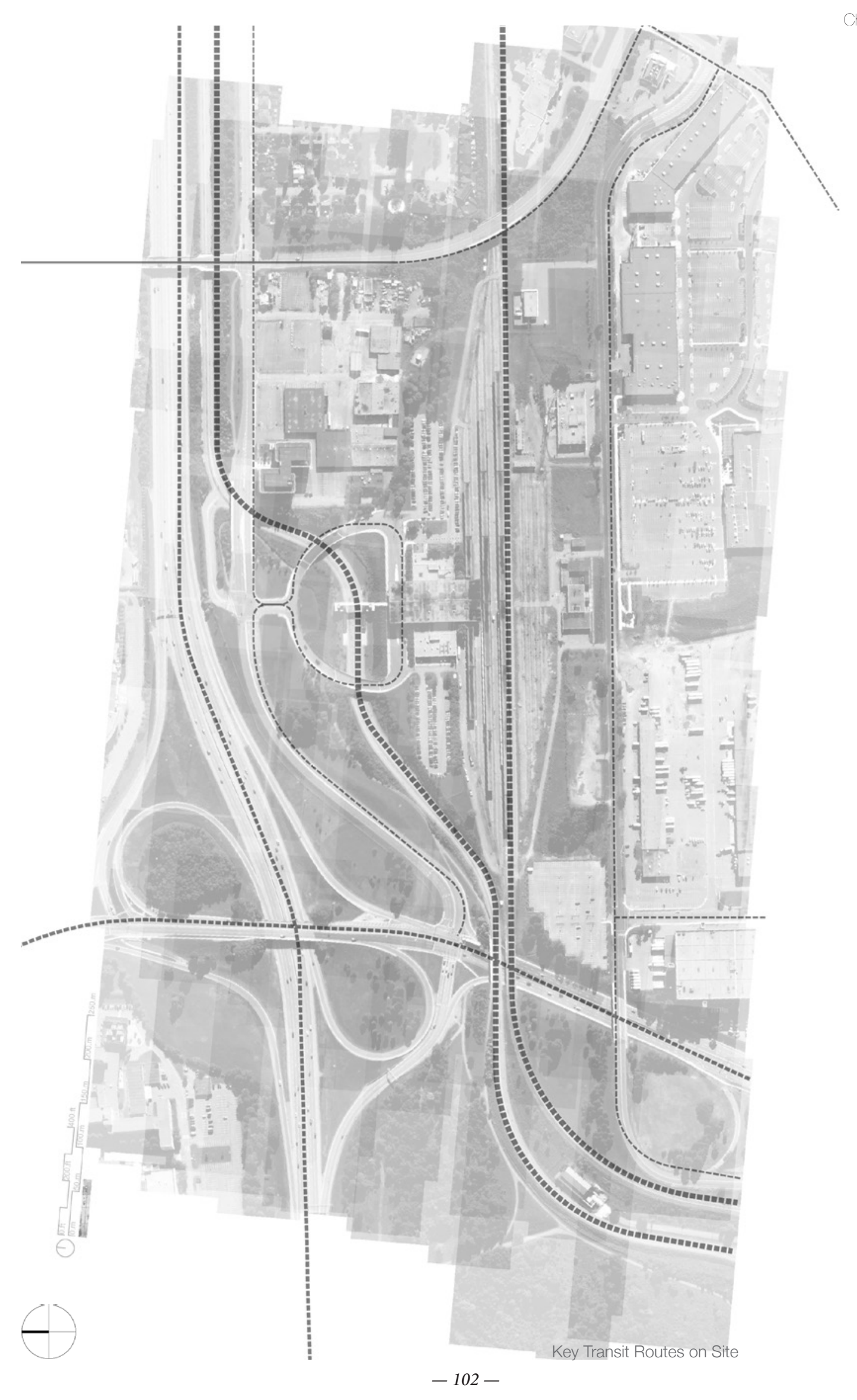




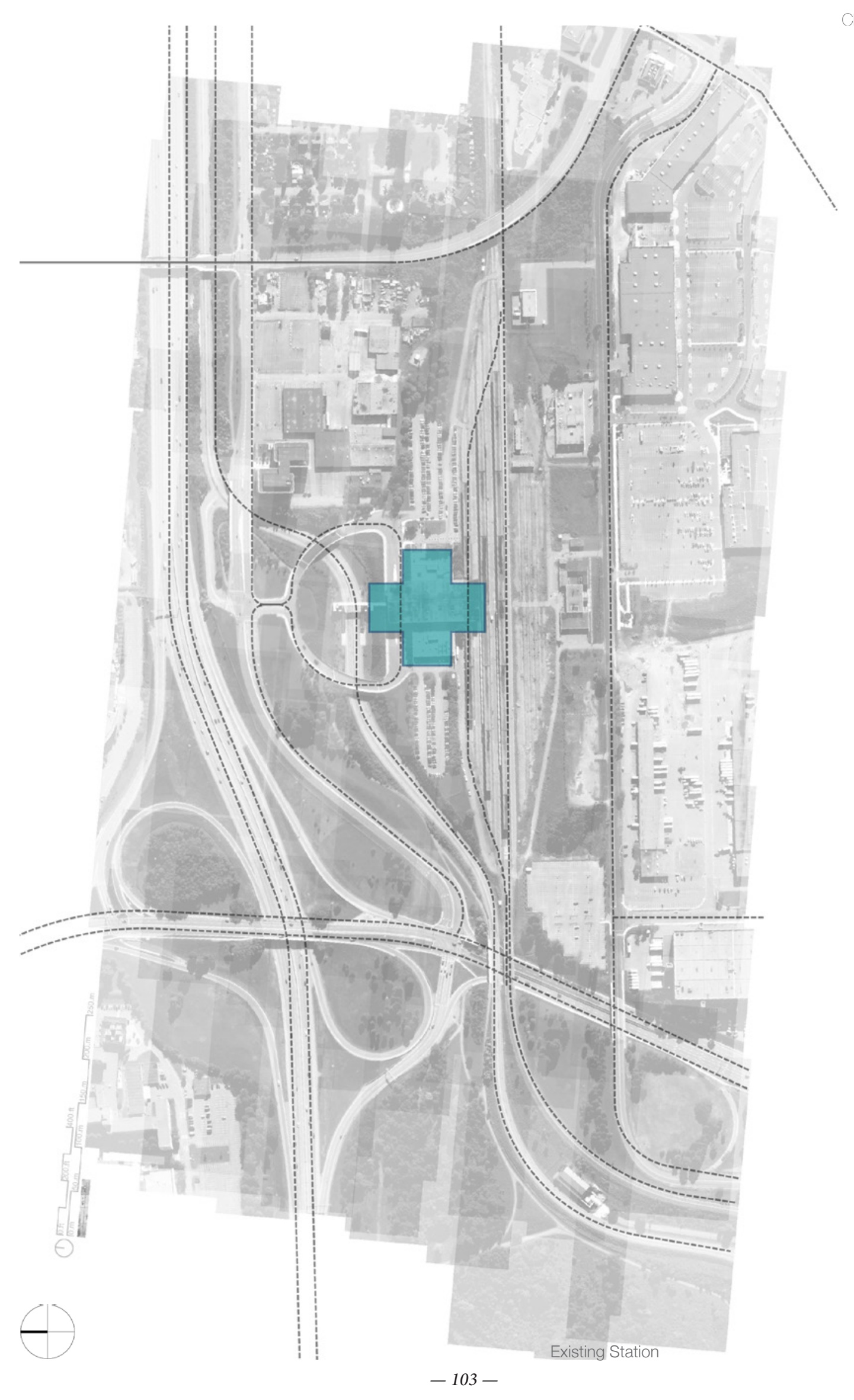




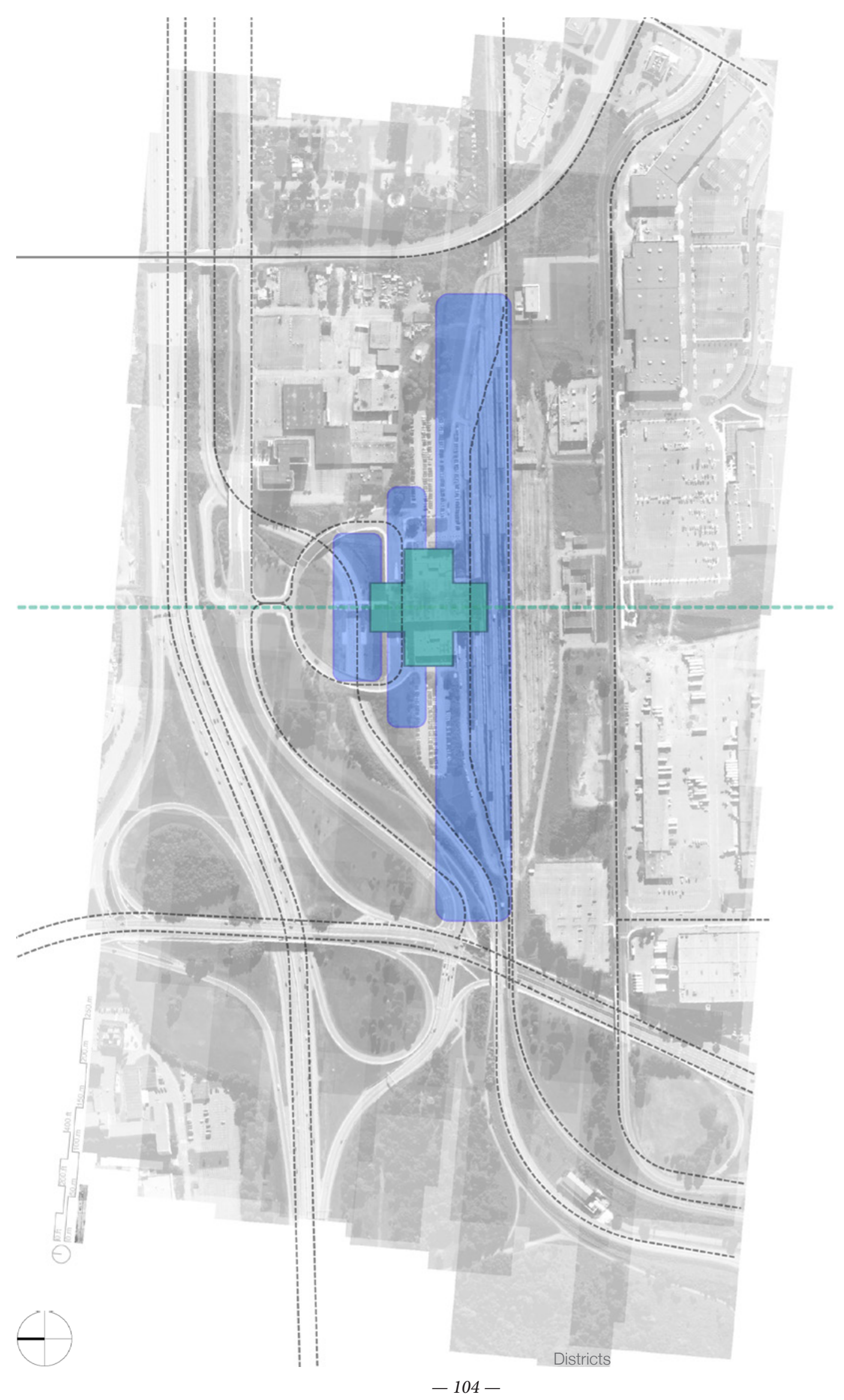




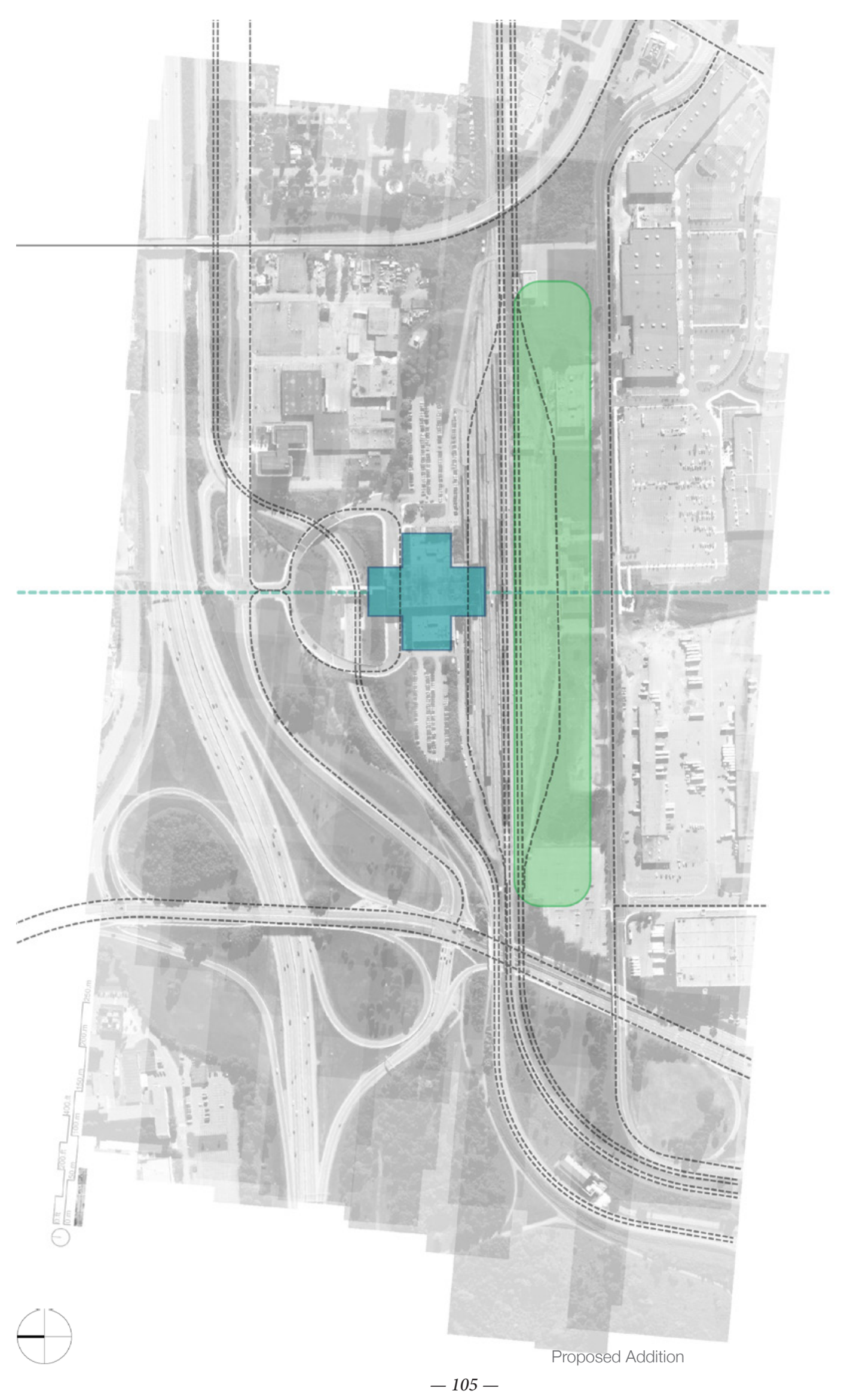

Chanter 4 


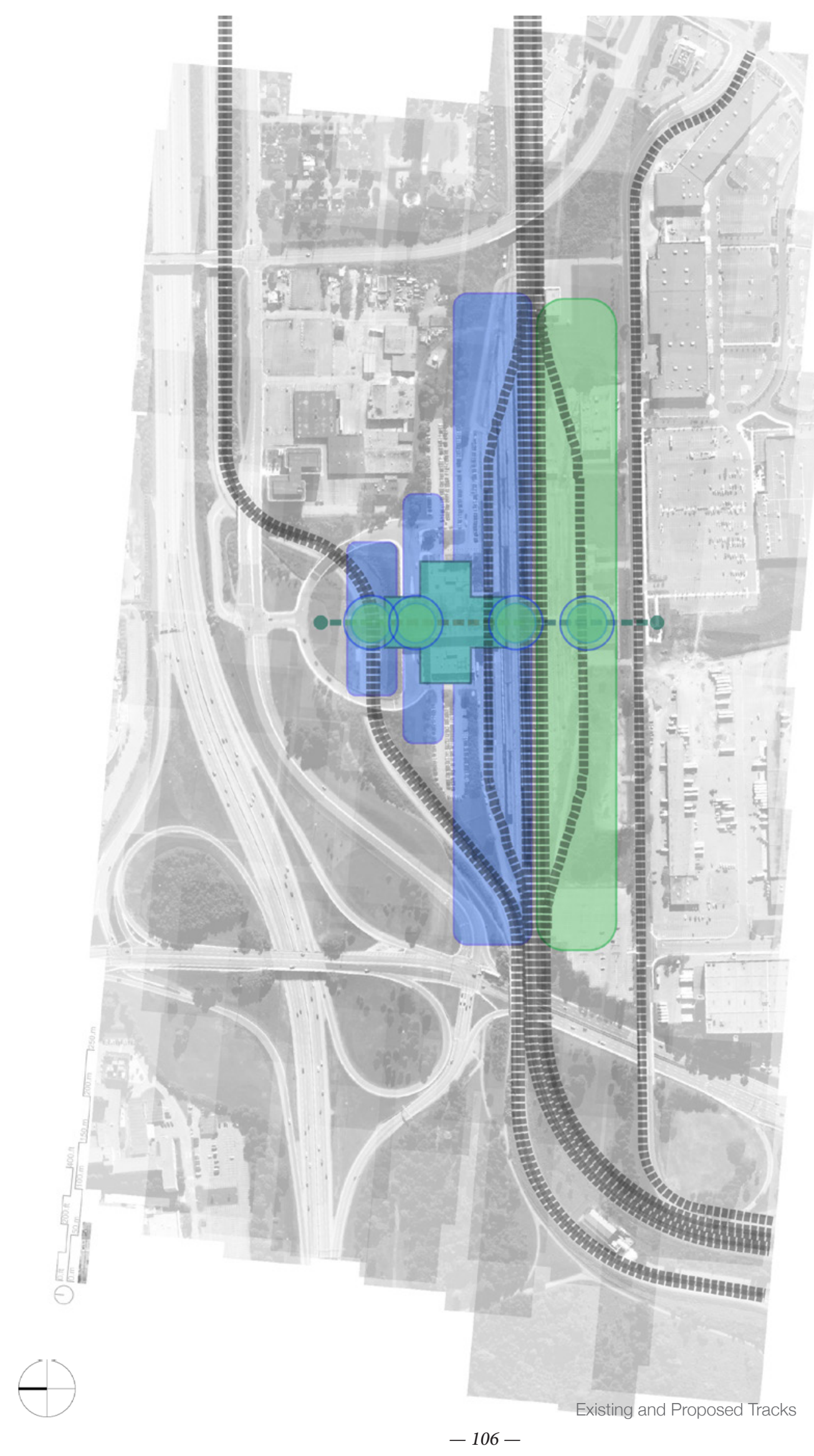

Chanter 4 


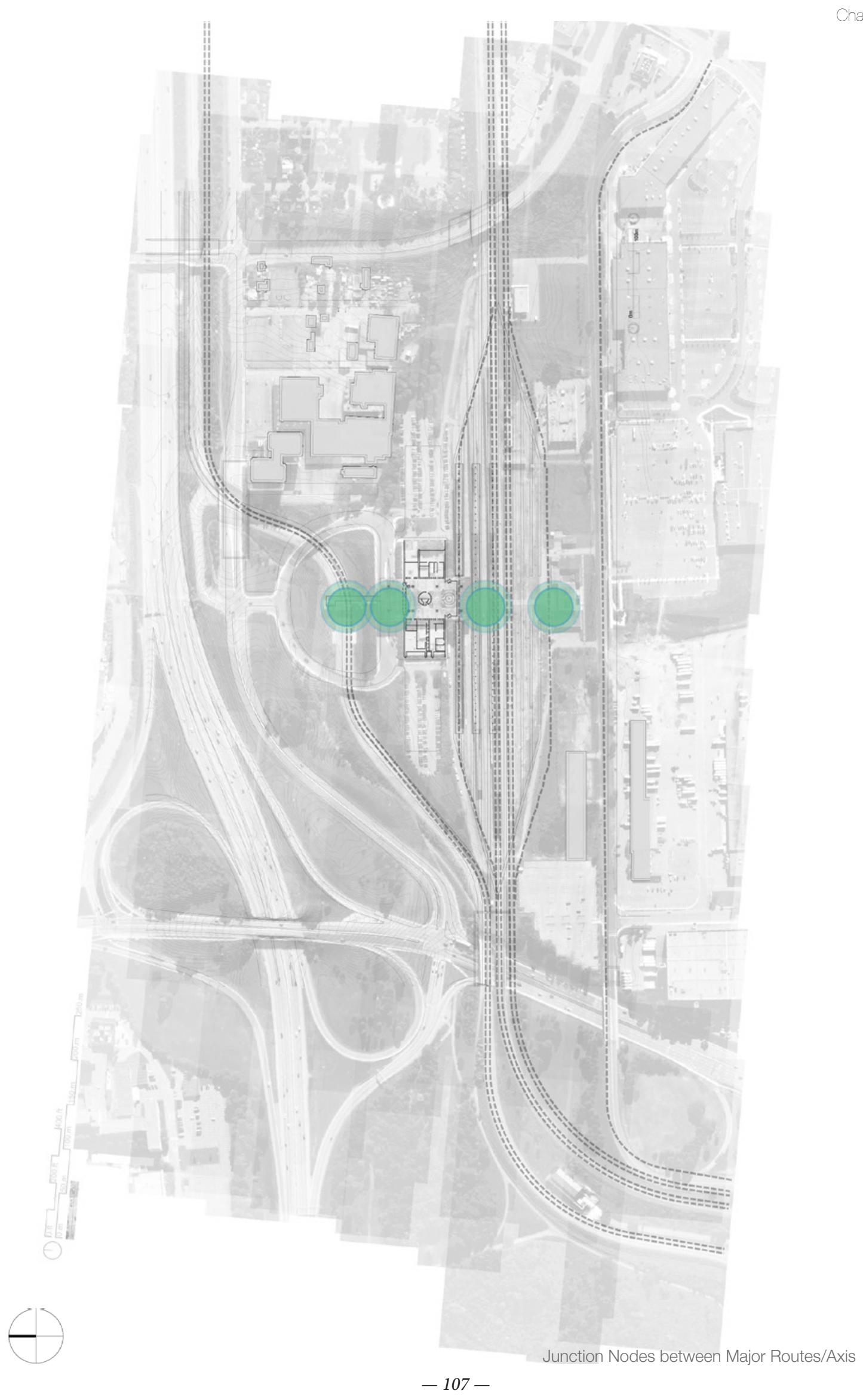




\subsection{INVESTIGATION III: SITED PHENOMENA}

The investigative phenomena has so far been isolated within its own autonomous "site" as an object. In developing a site-based contextual approach to the Ottawa Train Station, the next part of this line of inquiry suggests attempting to appropriate the processes found in the localized system. While the 'growth' could be interpreted metaphorically in terms of site, it is more useful to consider the 'act' of the phenomena and how those properties translate associatively.

In constituting this system as a series of nodes, the site works as a series of relationships to one another rather than individualized objects making up a composition. The node placement therefore establishes the primary acts of architecture and the subsequent parametric relationships.

This analysis bridges the relevancy of an overtly data-driven, scientific methodology in growth prediction to form a relationship with the identity of a meaningful wood material culture for the greater Ottawa region. Both inform the final placeform between sited traffic movements.

The phenomena was reinterpreted as site conditions to generate associative drawings, each increasing in complexity and expanse by iteration:

1) Metaball

2) Erosion

3) Drop Point

4) Expansion / Contraction

5) Field Network 

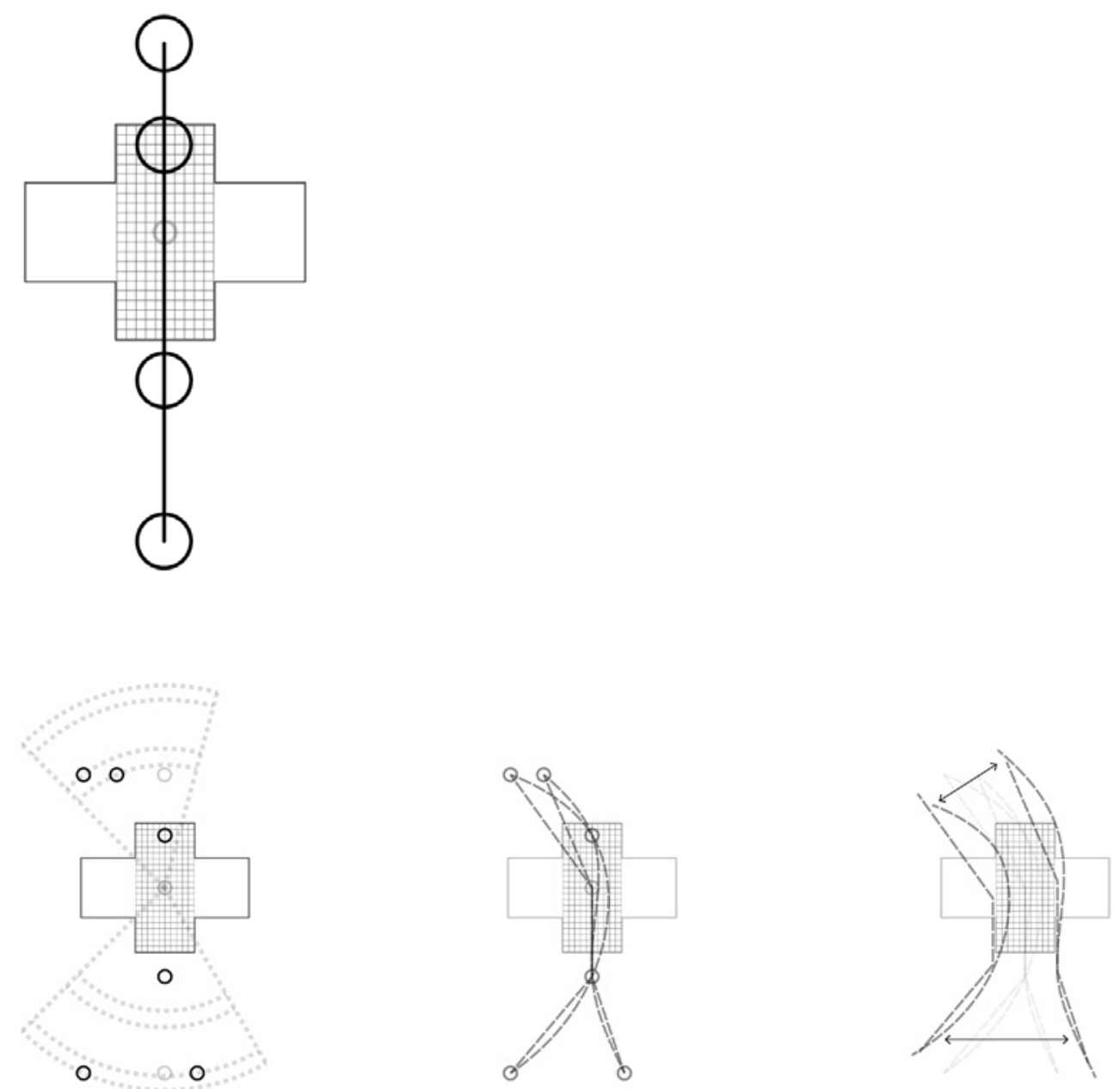

Node placement was determined by:

1) finding the space between key traffic flows on-site, 2) extruding these extents radially to establish a identical path of shortest distance through the center of the site, 3) connecting these paths directly with Nurbs curves, 4) extending the curves outwards to define general extents, 5) creating an amalgamated diagram $(R)$ showing the three established paths with their associative edges, nodes and districts.

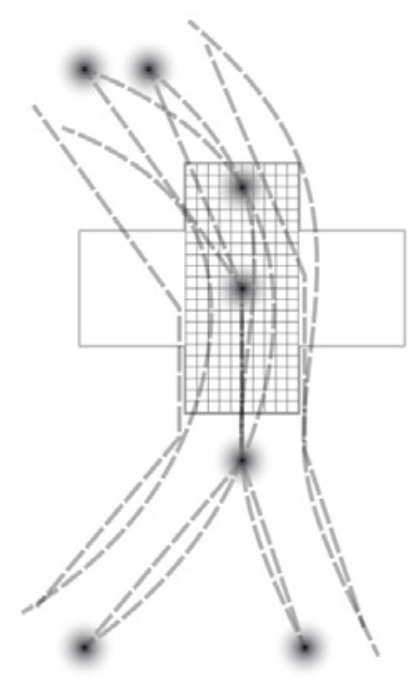



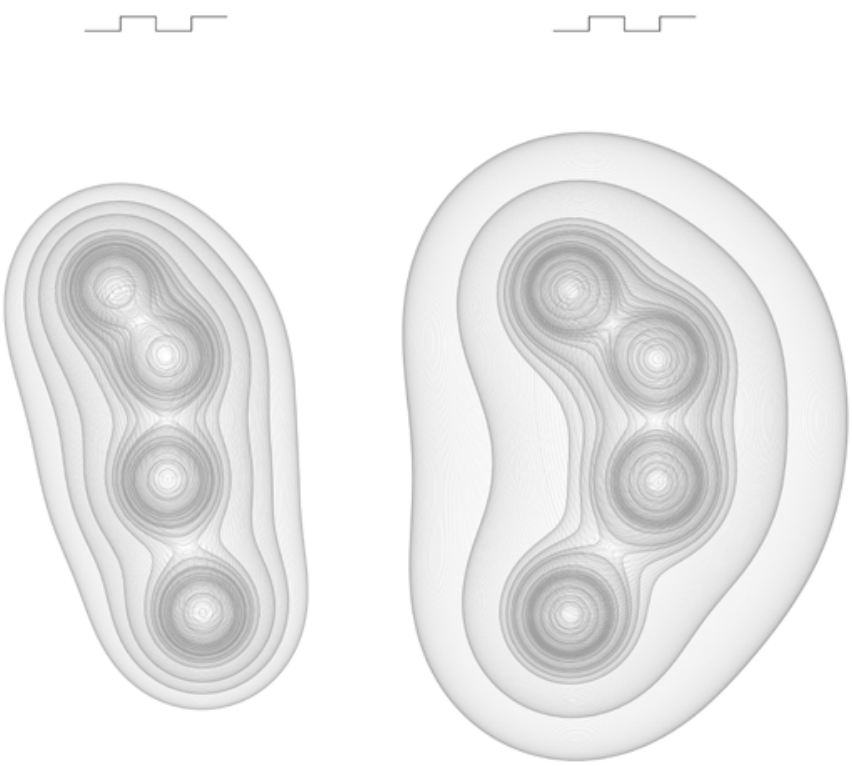

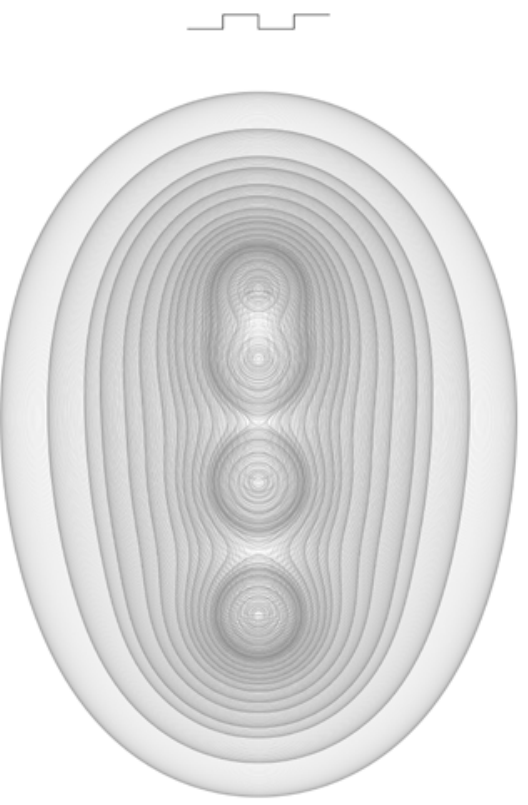


Chapter 4

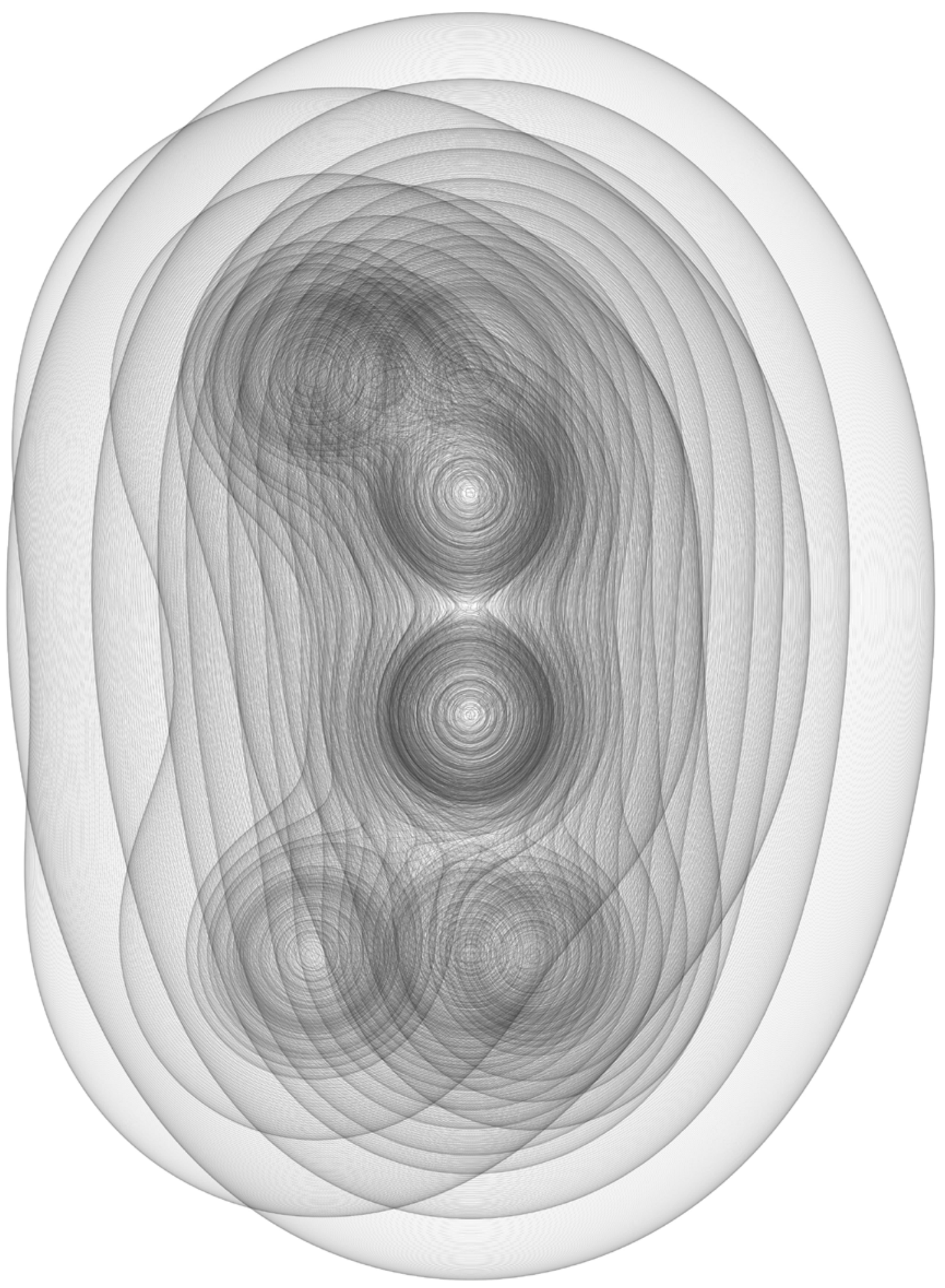

$-111-$ 

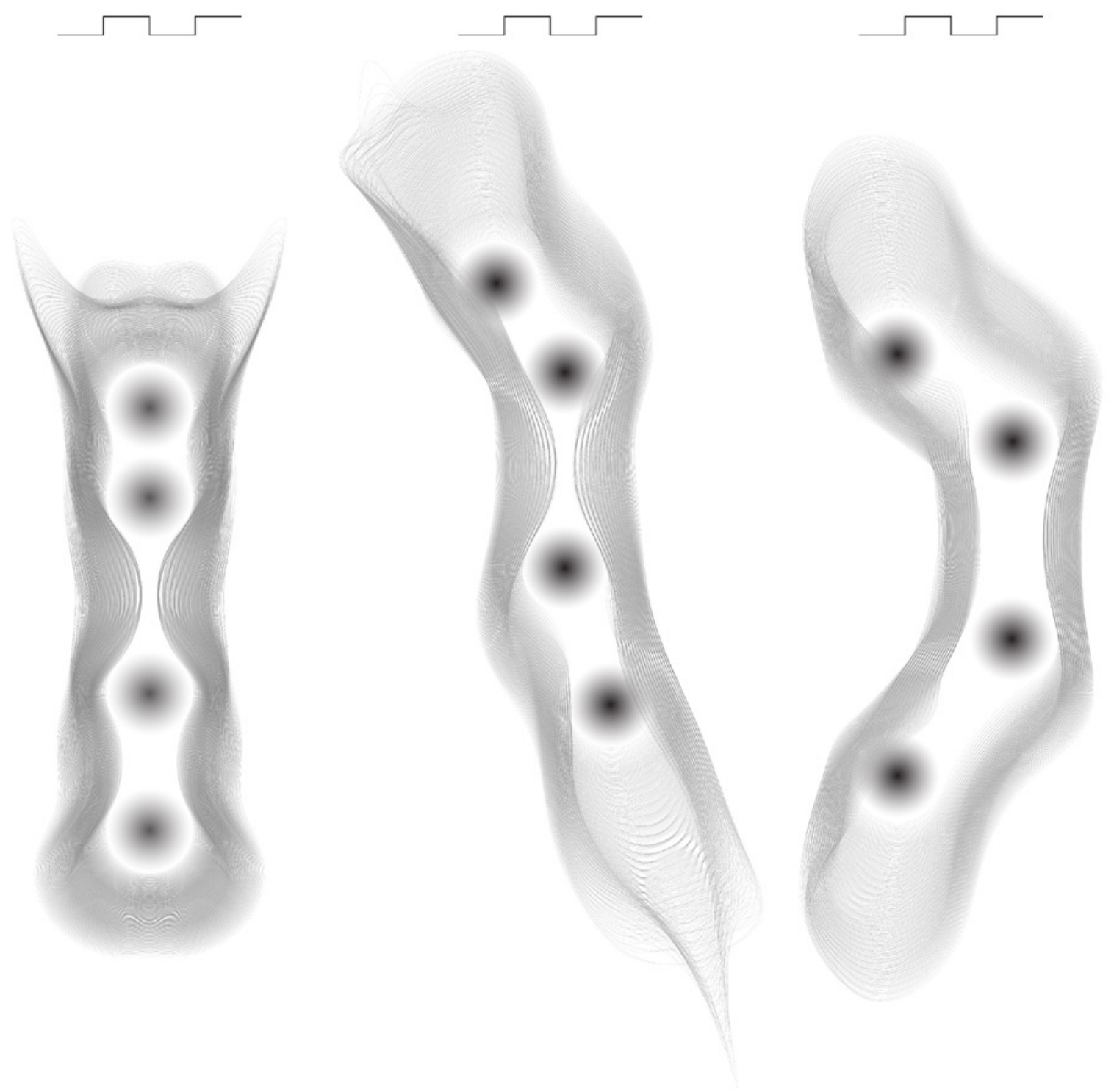

2) Erosion - extending established traffic routes to carve out areas where constant movement is situated.

$$
-112-
$$


Chapter 4

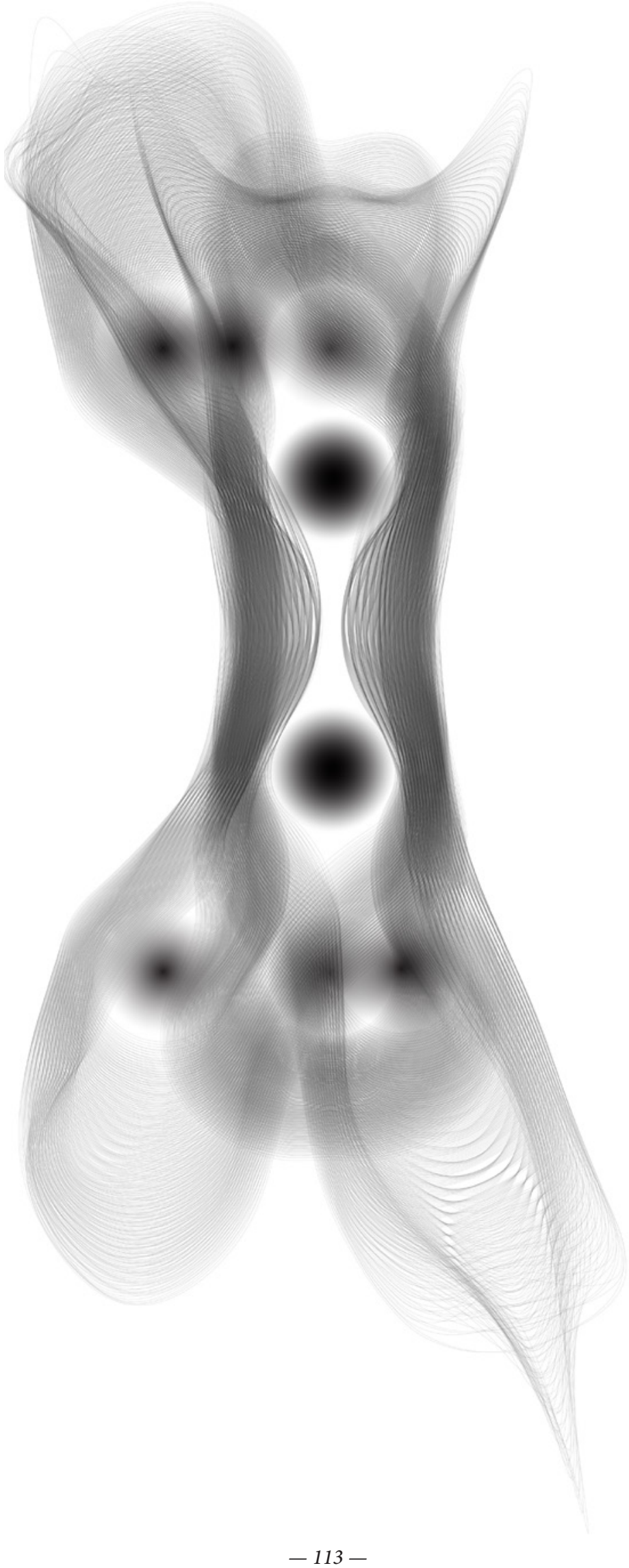



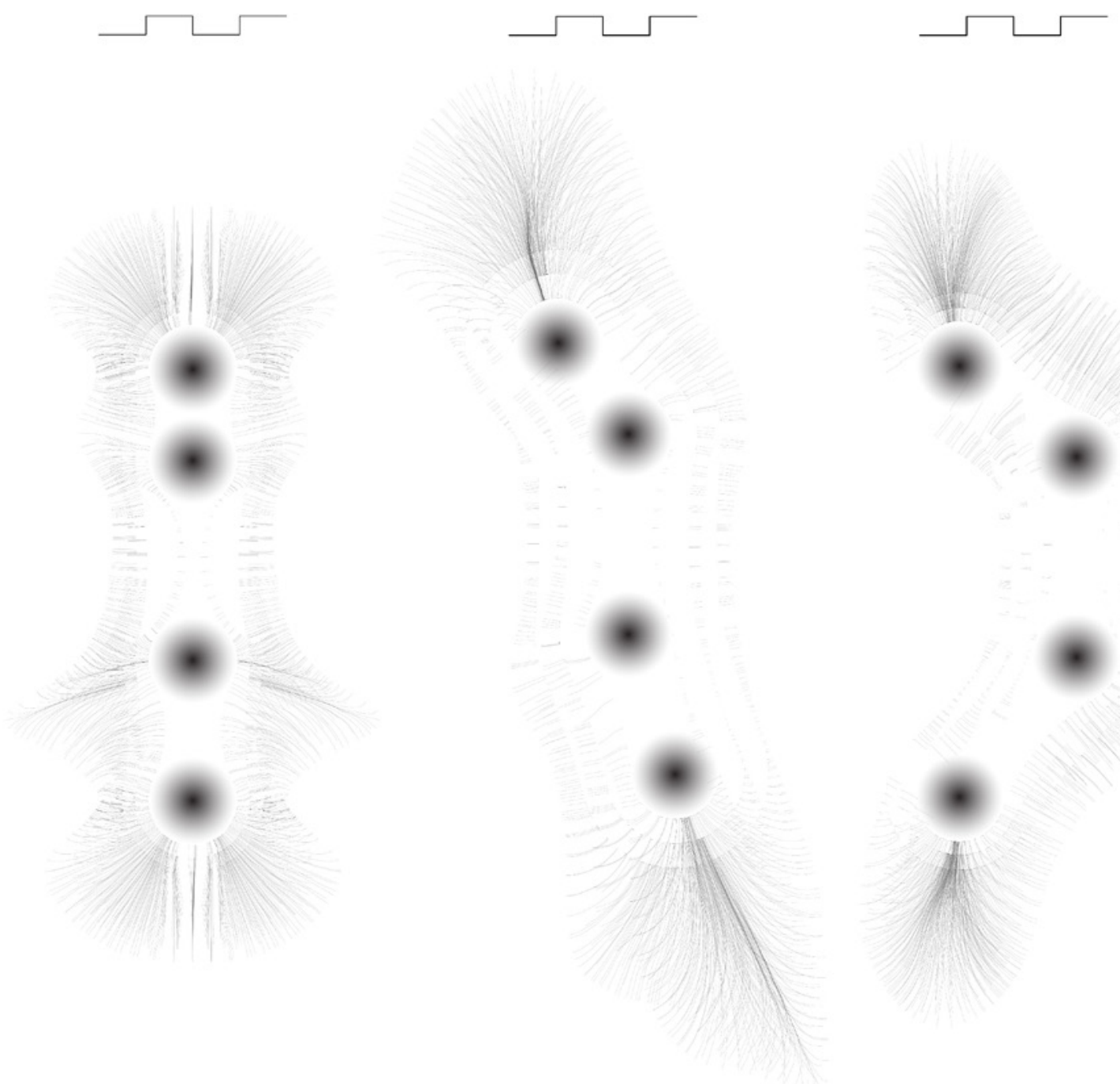

3) Drop Point Simulation - using the previous erosion simulation to create a surface that allows a grid of dropped points to depict the associative direction of movement towards the nodes.

$$
-114-
$$




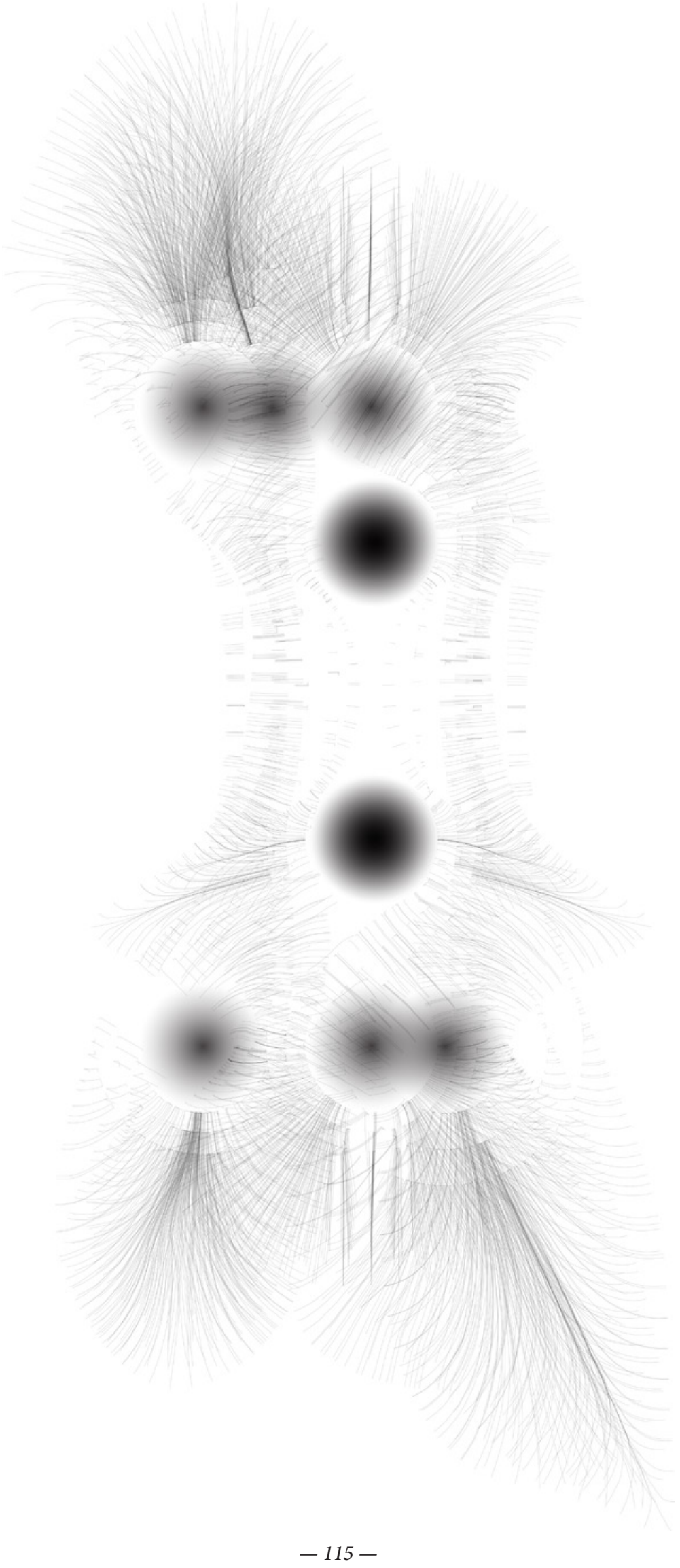



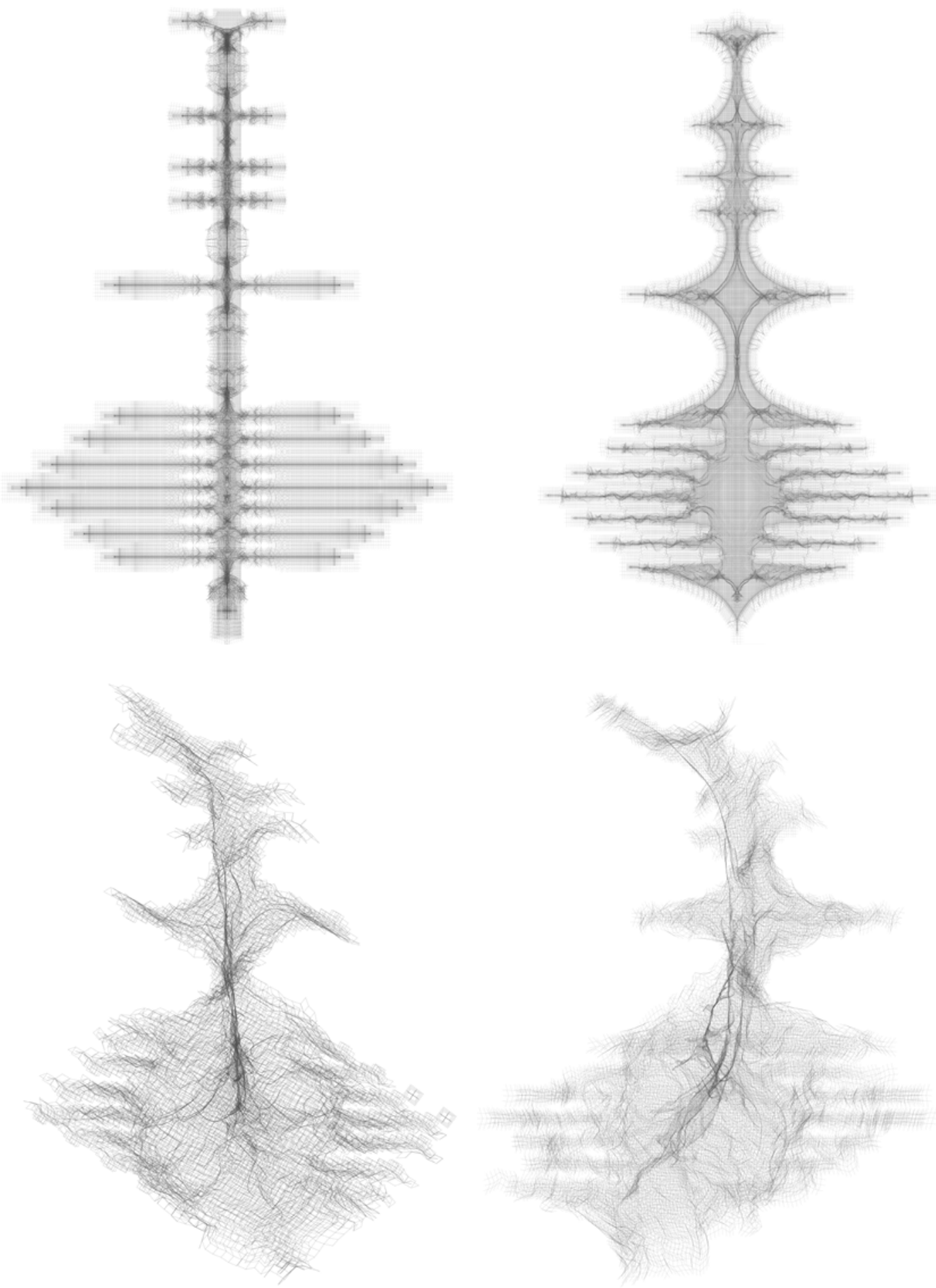

4) Expansion / Contraction - Generating a mesh grid that is pulled toward the center of the nodes, with the recorded movement overlaid to find resonance patterns. 


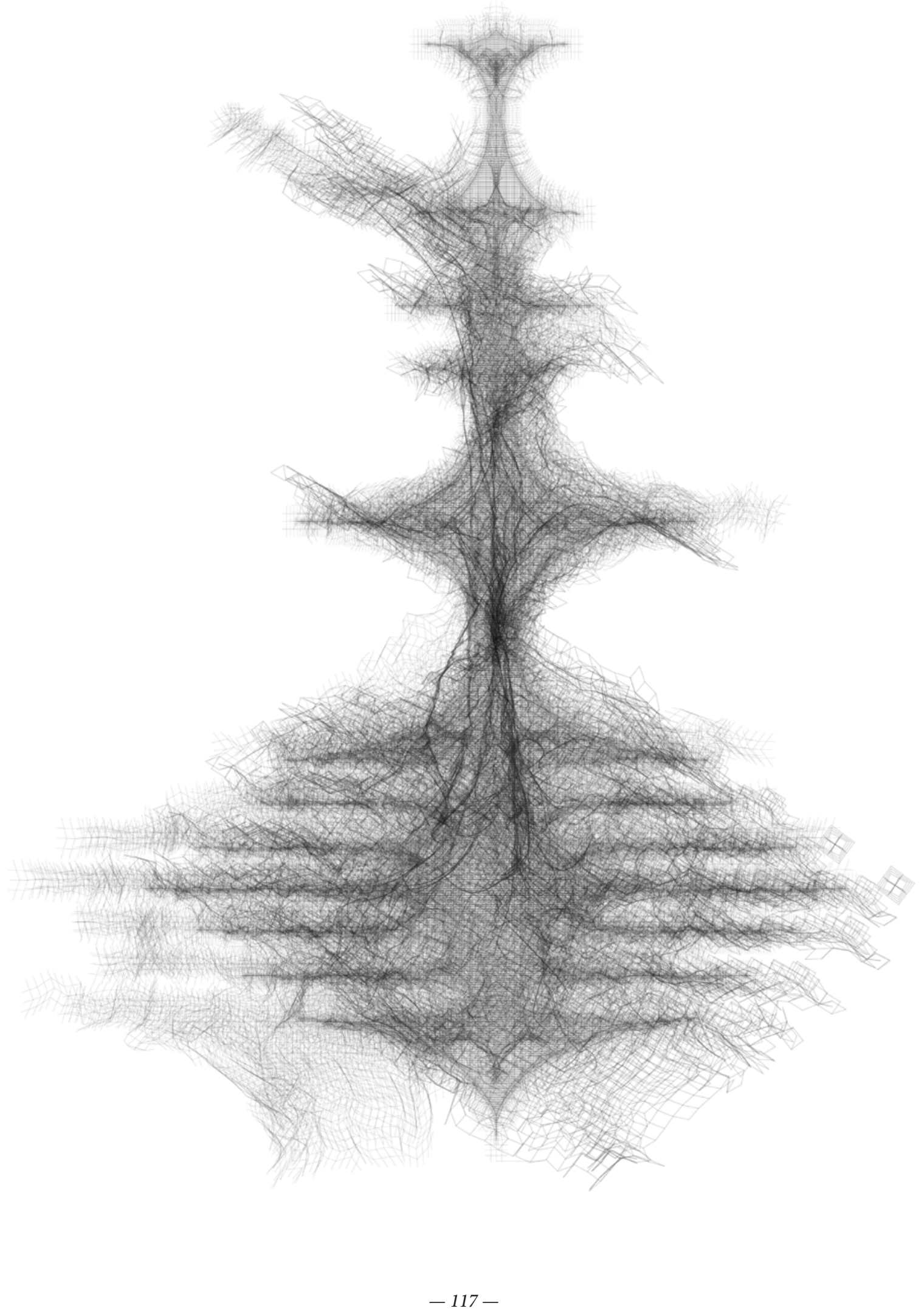

Curved Expansion/Contraction Detail 

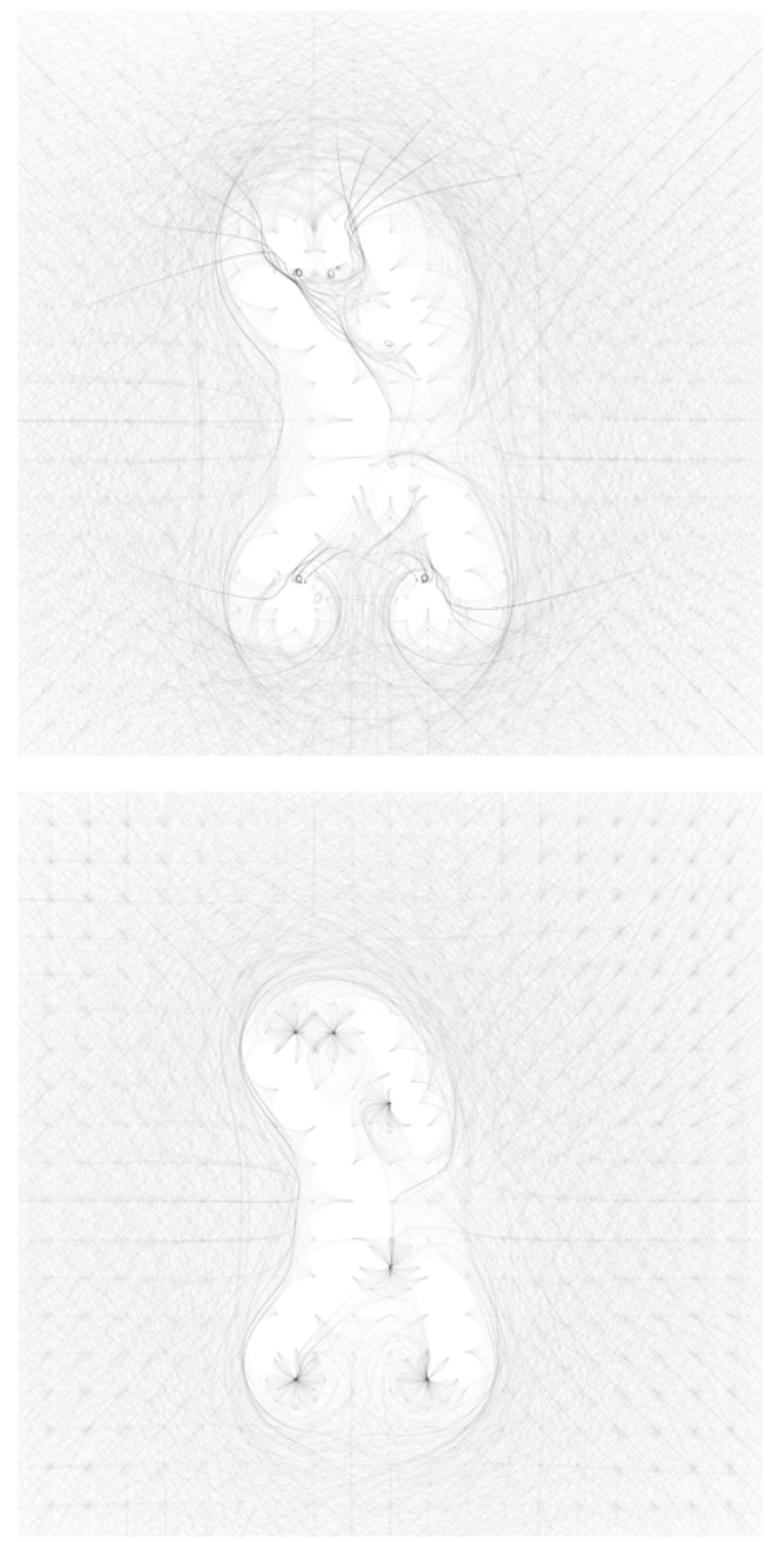

5) Field Network - Creating a field of outward (T) and inward (B) vector associations and recording their movements in relation to their surrounding components.

$-118-$ 


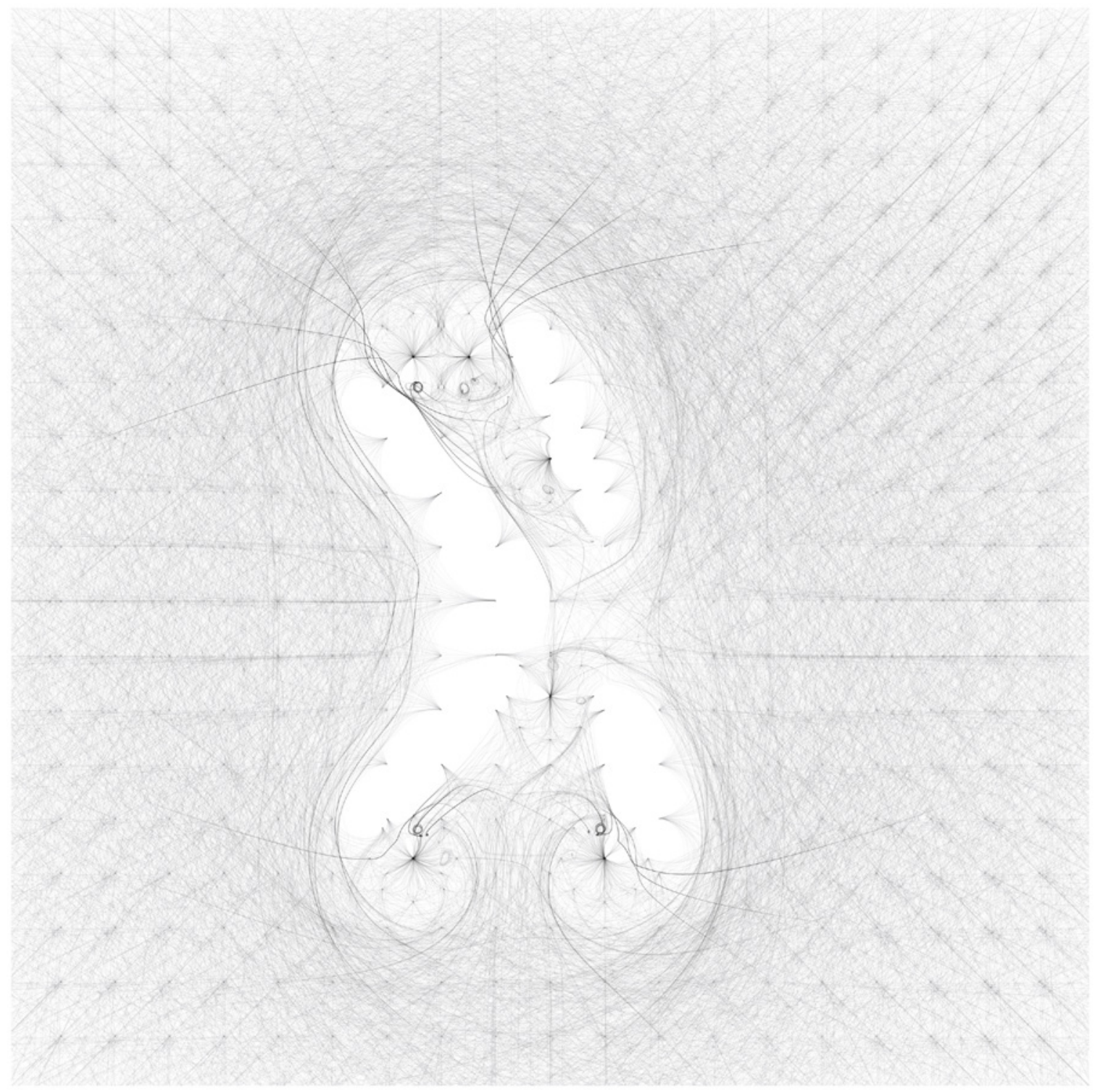

$-119-$ 

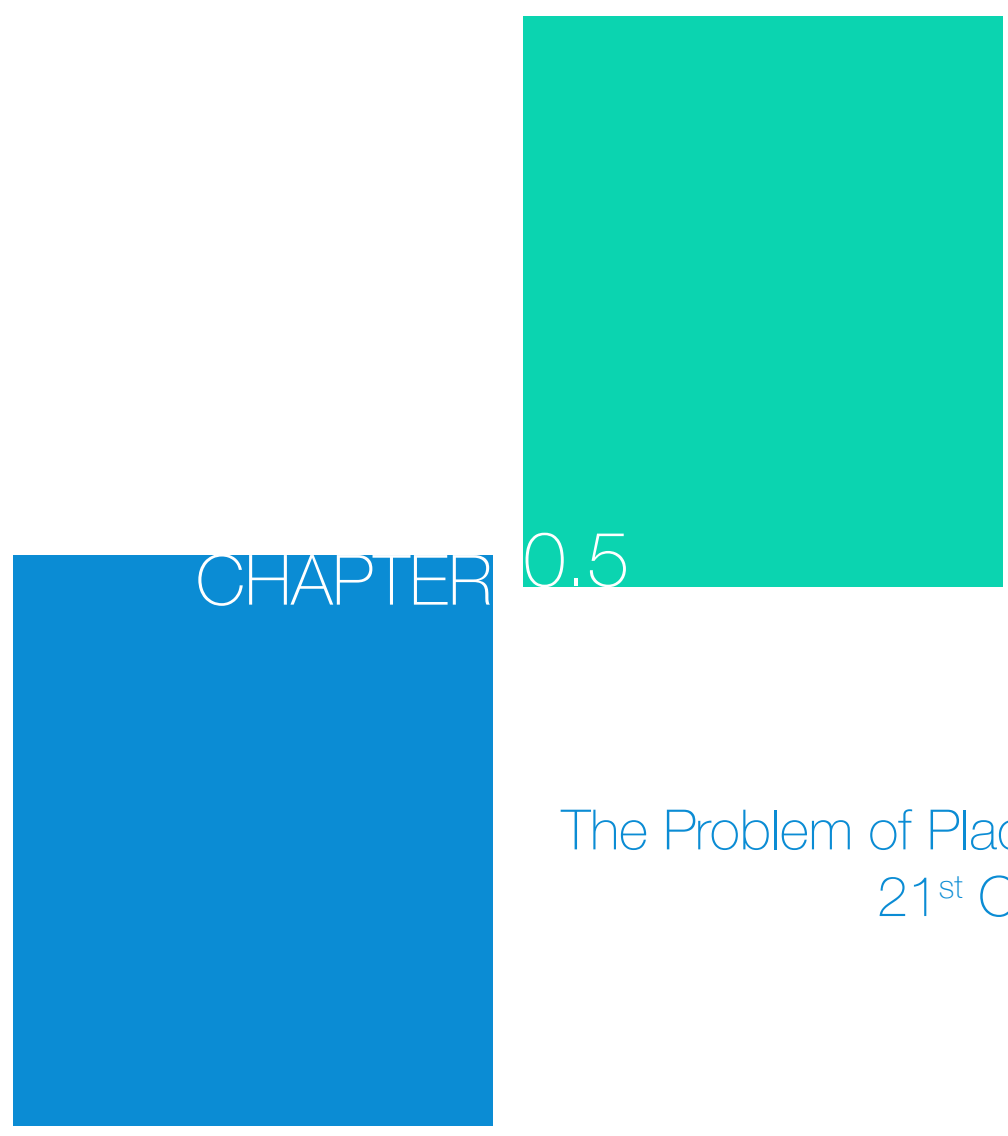

The Problem of Place and Identity in the $21^{\text {st }}$ Century 
"We think you're crazy to make us write an essay telling you who we think we are. What do you care? You see us as you want to see us - in the simplest terms, in the most convenient definitions." _ Brian Johnson [The Breakfast Club] 


\subsection{TRANSNATIONAL PRACTICE}

What does "place" mean in the era of architectural design where the computer reigns supreme? After all, globalized networks have expanded to inform methods of best practice in offices all over the world. It is now common for an architect in Ottawa to use the exact same software suite as one in Tokyo, Lisbon, or Oslo; practices must use the best tools at their disposal to achieve their ends. This method of best practice now transcends borders as a byproduct of the contemporary move towards globalization. A line drawn with AutoCAD in Ottawa is the same as the one drawn in Tokyo, Lisbon or Oslo. "The Digital Revolution is changing not only the nature of architectural production, but also its location. And this, in turn, redefines the connections of architectural production to the cultures of particular regions." ${ }^{1}$ This essentially outlines the new schism of regional building - locality is no longer inherently bound to a defined materiality or technology of construction, or even a sectarian method of drawing. This shift means that the "waning of affect' is in part visible in the collapse of regional identity and a corresponding loss of a sense of place."2

When talking about 'place' as it relates to a regional identity, it is often framed in the sense of Christian Norberg-Schultz's theories of the genius loci as an inherent 2009), 15.

2 Allen, Stan. Points \& Lines. (New York: Princeton Architectural Press, 1999), 14. 
endowment to the land it is located on. In this sense it is

a physical relationship to its surroundings between "the

distinction of natural and man-made phenomena, or in

concrete terms between 'landscape' and 'settlement' ${ }^{3}$ as a synthesized object.

As architecture moves away from theories of the 1980's, the idea of 'place' has remained ambiguous. Architects remain assertive that there are 'places' and 'nonplaces', it's difficult for many to describe exact criteria for categorizing into those denominations. General consensus has remained relatively steadfast to Norberg-Schultz's description that "Man dwells when he can orientate himself

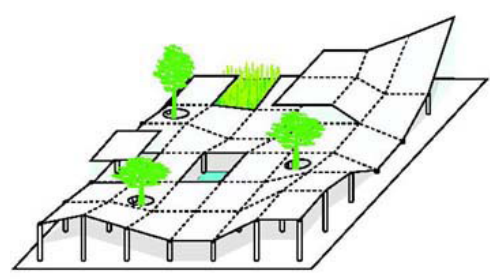
within and identify himself with an environment... when he experiences the environment as meaningful... The space where life occurs are places"4 because, as a relationship 'between how we do' and what we are as an identity, it reflects people on its most basic levels. "Identity is... our relationship to place"5 because it is elementally linked to our dwelling in the land via Norberg-Schulz's genius loci.

\subsection{THE TYRANNY OF PLACE}

The question of a sense of place does not need to be firmly embedded solely in what exists on site, however, as a limiting factor to create a boundary of what can be a 'place'. Rather, the theorist Fred Rush points out 
that there are in fact two fundamental ways to consider

making place: Firstly, as a strict response to totemic site conditions where "the project is supposed to be sensitive to existing... elements that surround it" such as Falling Water. The alternate, however, is to consider how "sitespecific' can refer to how the site is reorganized in terms of the building"7 as a way of establishing a place. This removes some of the onus from the site condition and instead gives more creative license to the designer. "Truly great buildings tend to go beyond site specificity; they are almost site determining," 8 that is, where Norburg-Schulz prescribes the architecture as fitting into the landscape, primacy can also be extended to the landscape fitting to the building. The previously established idea of place is now being questioned further; why should place be relegated solely as an offshoot of some kind of elemental existence? The Norberg-Schulz "place" as a form architecturally endowed to its site proposes a method of continuation between landscape and building. Mari Hvattum, however, points out that this method of thought is in many ways producing mere imitations of nature, where man-made objects are simply ceding authority to the natural. Her criticism of the established Norberg-Schulz line of thought is the over-reliance on a pseudo-natural approach to place that has not changed since Norberg-Schulz's writings. Instead she proposes a new architectural definition of place for a younger generation coming into age in a

Fred Rush, On Architecture (New York: Routledge, 2009), 110.

Ibid.

Rush, On Architecture, 120. 
contemporary discourse that has embraced the benefits

of the digital. This distinction is similar to the rejection of mimicry in turning towards a phenomena-based approach. In this, "[the] denouncement of the local ground and the invocation of wider horizons for their architecture could be read as a programmatic statement for a new generation of... architects rebelling against the 'tyranny of place."'9 Her 'tyranny of place' is an apt method of describing what parts of the discourse have devolved surrounding place. It is notable because this 'tyranny of place' is completely different from 'placelessness' or 'non-place,' which are much more commonly written about as interchangeable with poor architectural spaces. Rather, she rejects the inherent assumption within Norburg-Schulz's argument that place is dependent on a prescribed natural relationship to earth. Hvattum points out "how can something as artificial and complex as a building be described as 'natural?"'10 This is particularly relevant in contemporary discourse, when the complexity of any building type has expanded exponentially with technological advances becoming embedded further within them. Her 'place' recognizes that contemporary design is much further evolved from the vernacular huts of Norberg-Schulz's Norwegian countryside. The meaning embedded within the ground has evolved since the proposal of genius loci. Instead, the concept of place has moved on to "cultivate a sophisticated and many-layered artificiality, made

9 Mari Hvattum, "Making Place" in New Nordic: Architecture \& Identity (Denmark: Rosendahls, 2012), 107.

10 Hvattum, "Making Place," 103. 
meaningful in contrast to nature and place more than as

its continuation or imitation." 11 To instill meaning into a contemporary idea of place, as Hvattum is arguing, means to break free from those tired ideas.

Touching on similar themes of clarity and contrast, Lynch describes "if it is our purpose to build cities for the enjoyment of vast numbers of people... we may even be wise to concentrate on the physical clarity of the image and to allow meaning to develop without our direct guidance." ${ }^{12}$ According to Lynch, 'meaning' (as an extension of 'place') may be more related in the way that it develops a clarity of visual perception rather than as a personal bias projected from the designer onto the building, and in turn those who come into the space. This line of thinking may act as a safeguard for the 'placeless', something that appears to happen largely when the designer projects a singular meaning into his own design that resonates for himself (the designer), but no one else.

In tandem with describing the placeless, Lynch also describes an analytical method for helping to form place in the world of the user. By creating a mapping of a space, either through visual clues or a physical method, the designer "can provide the viewer with a symbolic diagram of how the world fits together... as long as he can fit reality to the diagram, he has a clue to the relatedness of things." 13 Even though this relatedness of things might seem to only apply to intimate situations, Sverre Fehn points out 
that this can mapped association can be extended to

man's existence on the horizon, as "the simplest form of architecture is to cultivate the surface of the earth, to make a platform. Then the horizon becomes the only direction one has, and the moment a dialogue between the earth and the horizon is established, one can start to consider it as a room." ${ }^{14}$ The ability to mentally map a space by constructing it perceptively based on visual cues allows the individual to establish a deeper connection to the space, because "map reading, research has shown, strengthens our sense of place and hones our navigational skills."15

\subsection{PLAIN CONTEMPORARY}

The Norwegian firm Snohetta has steadily increased their professional profile, both in architectural circles and to the general public, since their founding in the 1980's through the completion of a number of successful ventures. In current terms they now work on projects across the world from offices in Oslo and New York with their uniquely Nordic approach. They have published two books concerning their approach to practice, often touching on identity in relation to a globalized profession.

Through their publications, Snohetta has outwardly questioned an "explicit skepticism towards the notion of the genius loci"16 proposed by their fellow Norwegian. This

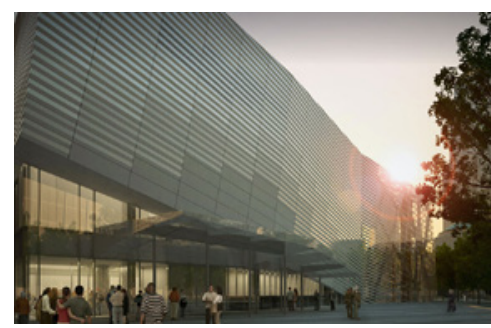

14 Fjeld, Per Olaf. Sverre Fehn: The Pattern of Thought. (New York: The Monacelli Press, 2009), 108.

15 Carr, Glass Cage, 129.

16 Snohetta, Conditions (Germany: Lars Muller Publishers, 2007), 60. 
seems counter-intuitive, as the firm has been responsible

for some of the most identifying, iconic buildings since the turn of the millennium. Among them: the National Opera House in Oslo, 9/11 Memorial Museum in New York, and the Alexandria Library Egypt. In the Canadian context they have completed the Bader Arts Center in Kingston, Ryerson Student Center in Toronto and are currently working towards completion of the Calgary Central Library.

To Snohetta, the problem of place has become too ambiguous, as 'genius loci' has slowly become a catch-all phrase $^{17}$ that can seemingly be pertinent to any situation where 'place' has a rootedness in the existential idea of humanity in the landscape. This position means that 'place' as a landscape rootedness may be a falsehood, at least in an autonomous pure sense. This seems accurate, as most contemporary successful 'places' are never proven before construction, but rather after completion. Place is not postrationalized, but it is post-realized - and this always has to do with how people interact directly with it. If that is true, then the problem must be reframed: "Anything may be argued with reference to the genius loci of a place, except the most vital: What human conditions must be at hand to recognize this implicit identity of a place?" They answer this question with three conditions: 1) Instinct and reflex, 2) Perception and emotion, 3) Science and research. ${ }^{18}$ They are most interested in creating place by how people actually interact with the natural phenomena of landscape. 
They call this the 'non-place' for how their distinct places

are projected onto the user's mind, ${ }^{19}$ and incorporating landscape with nonscape becomes their viable strategy to achieve place. It suggests that translating NorbergSchulz's mediation between human and landscape into contemporary digital practice has much more to do with mediating how human and nature act. This promotes a working method to produce what is "associative rather than symbolic." 20 Allowing a design process that focuses on the actual way that the inhabitants grow into the space reinforces a building form that is dictated by and reinforces programmatic constraints. Structural freedom is used to express identity via direct usage, as "the directional flow and movement in urban hubs dictates built form as much as it does urban form." ${ }^{21}$

Their contribution is notable for the fact that, while pursuing a process heavily indebted to the longstanding Norwegian appreciation for Modernism's traditions with intimate material conditions, they incorporate this with a rigorous digital process in production. This allows them to harness the inferential power the computer can provide in supporting their working method while also avoiding a false complexity in the final product through a grounded phenomenology of design. They are able to find Pallasmaa's tension between the coherent suggested illusion and the credible imaginary world. This represents an idealized contemporary best practice free of the tropes found in

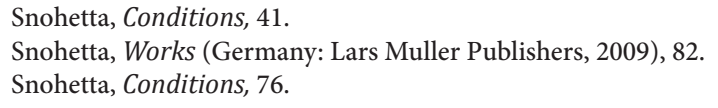


“-ism's."

\subsection{DIGITAL REGIONALISM}

'Parametric' has been applied colloquially to architectural design so broadly since the 2000's that the term has become practically meaningless - a descriptive word without agency.

By its mathematical definition, parametric simply means that multiple solutions are made available based on a variable input. In architectural terms, it relates primarily to a returnable, editable, self-perpetuating function for working through design development.

The term 'parametric' has been hijacked from its birth as a robust type of design tool to describe a 'style;' most prominently by Patrik Schumacher.

The problem is that 'parametrics' are not the sole propriety of the architectural world, or even that of the general art and design community. It is not the lone domain of recent advancements in these disciplines, nor is it exclusive to the digital. The most prominent reflection of this might be Antonio Gaudi’s Sagrada Familia Basilica in Barcelona, initiated in 1883, which used analog descriptive geometry and models to develop its form and structure, most notably through catenary curves. ${ }^{22}$

The overuse of the word 'parametric' has grown to describe anything created through the use of advanced

22 Gustavo Garcia Gabarro. "Nature in the Sagrada Familia." In Sagradda Familia: Gaudis Opus Magnum (Spain: Media Minds SL), 79-83. 
digital tools. Although this does encompass a node-based

methodology, it has become a contemporary misnomer. If something is 'parametric', it does not inherently make it digital, and vice versa. To describe it as a style is to suggest that the emphasis should be placed on the 'look' of using digital tools. The ambiguity of the term is precisely the reason why it no longer has any authority. Digital tools should instead be used for rationalizations to find a specific placeform within the context of the project, and let the 'look' be an extension of that.

The vernacular, in a parallel way, has seen a similar arc of usage. Originally a practical building method of local tradition and materiality, it was appropriated to scenarios that did not share the same external influences. In the appliqué of faux-vernacular motifs, an architecture of integrity and reflective identity got watered down to a stylistic device relying on the sentimentality of kitsch. Sincere vernacular architecture, in the same way as current digital tools, should promote a methodology that uses them with integrity to find an architectural end. The creation of identity and sense of place is therefore contingent on the integrity of their application, and therein lies their ultimate shared attribute.

The product of this is a methodology that can use accuracy and precision of systematic methods to create an architecture that responds to the various surrounding factors. This suggests that the relationship of the object to the surroundings hold the most importance; in other words, contemporary regional context reigns supreme. The by-product of this methodology is an aesthetic that 
projects its inherent responsive qualities to the users. This hierarchy of primary and secondary influence cannot be ignored. "Form matters, but more for what it can do than for what it looks like" - or more directly, "Form matters, but not so much the form of things as the form between things." ${ }^{23}$ Form does not follow function; the form is the function. Comparatively, it supports and enhances it - the 'use aesthetic' of the digital revolution.

The clear association of relationships between the separate parts, whether digital or material, must take the most prominent role in developing an architecture of identity and place. False complexity must stop masquerading as information that reinforces the ivory tower of contemporary digital practice - Schumacher's autopoiesis is giving way to Bjarke’s democracy. Digital universality can be incorporated with a regional contextuality to safeguard from the premium of the surface as a homogeneous design element without simply recreating the visual of the computation. That act is the new 'place' in contemporary architecture. To incorporate people not as passive observers but as actors of agency defines new place and recreate human conditions as subjects.

If the critical regionalists reacted to Internationalism with a site-based contextual approach, digital regionalists should react to the similar placeless homogeneity with a use-based contextual approach. This can be achieved by leveraging the use of digital tools rather than discarding them. Design as a conveyor of identity still 
must be grounded in real things to sustain an association to

place. This offers a new twist on the Frampton standard - a

Digital Regionalism for the 21 st century.

A) Plot Architects, Maritime Youth House Diagram, Nordic Identities, 114

B) Snohetta, 9/11 Memorial Render, Works, 186-7

C) Snohetta, Oslo Opera House, Works, 36-37 


\subsection{INVESTIGATION IV: DEVELOPED PHENOMENA}

The analysis developed to this point has applied to the site conditions but has remained largely abstract, suggesting schematic approaches. However, the same sets

of relationships can also be applied at the architectural and human scale for this project.

At the architectural scale the relationships between the nodes can also begin to define the placement of gathering areas, waiting areas, key movement areas, etc. Once these extents are defined, their spatial relationships can be focused and heightened in the context of the existing building. As the central nodes are placed to inform the main intersection of movement flows, those areas can become visual attractors to the surrounding areas.

General layout and floor planes are developed by using the phenomena patterns to produce movement diagrams that can be extruded into three dimensional landscapes of migration through the space.

The human scale can also be addressed through a digital process informed by simple regionalist placemaking processes. This considers craft and construction as a sequence of moves and translations that increase in complexity through series of parametric relationships. Traditional building methods can be dissected and reinforced to produce more complex assemblies. 

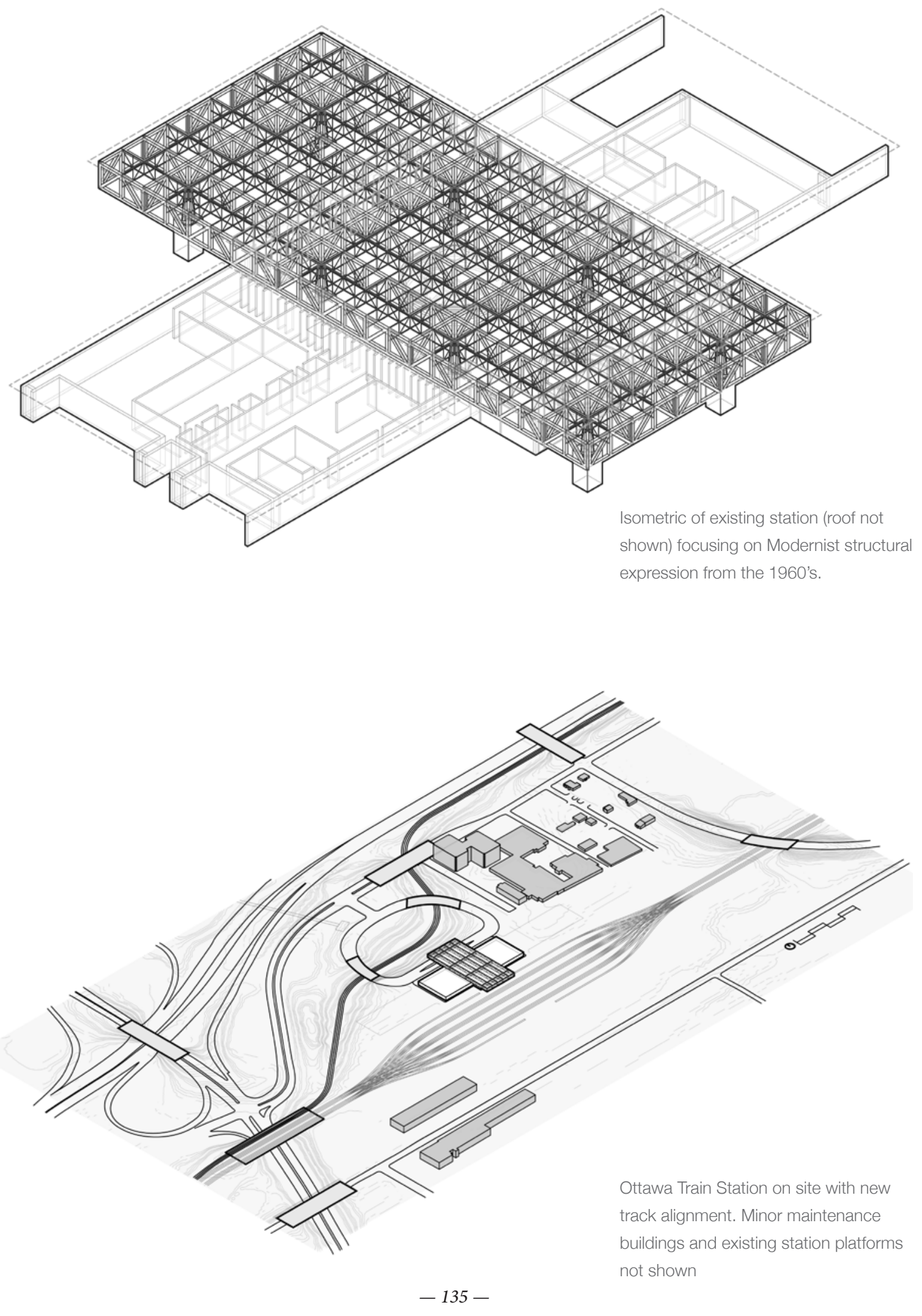


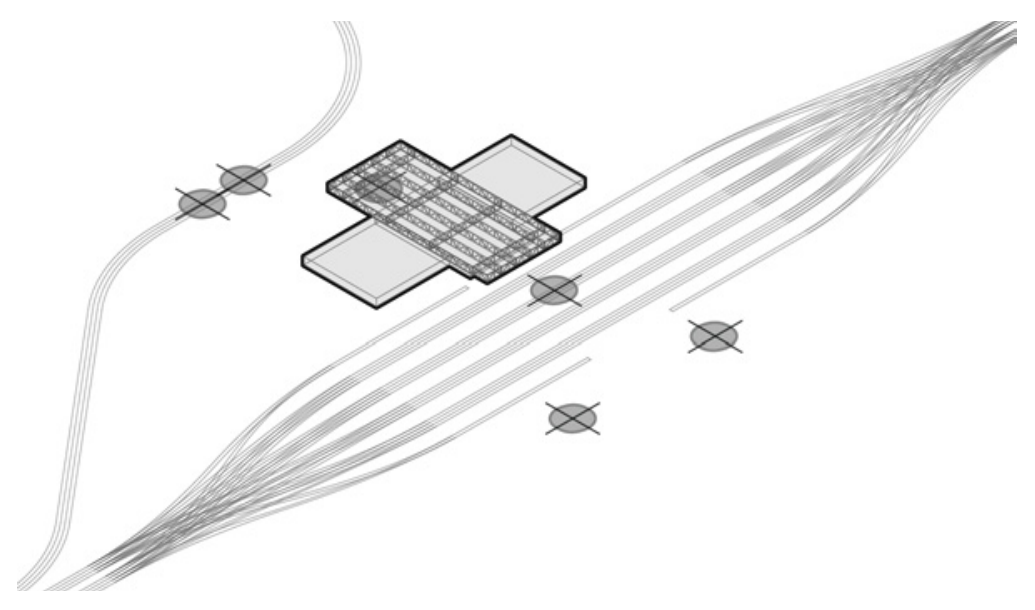

Chapter 5
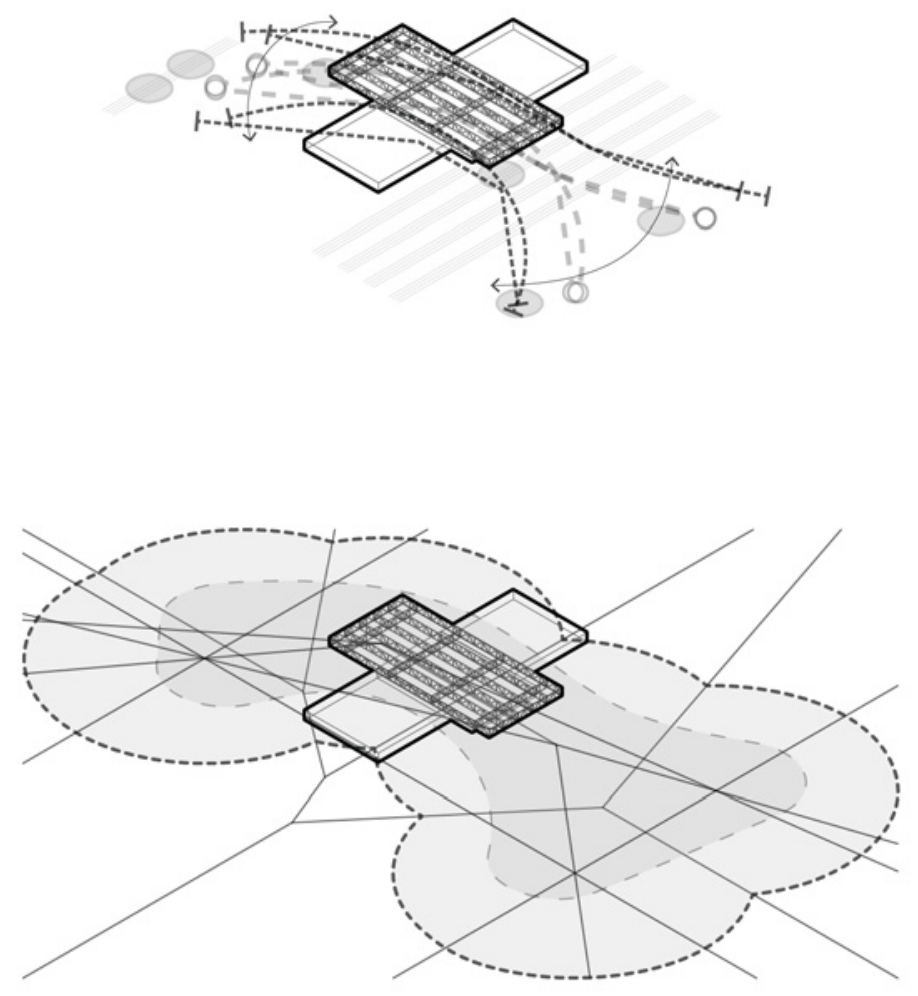

1) Building isometric with track routes

and node configuration.

2) Path/Edge/Node/District

3) Voronoi \& Delauney diagram showing

connectivity and separation patterns

between node points 

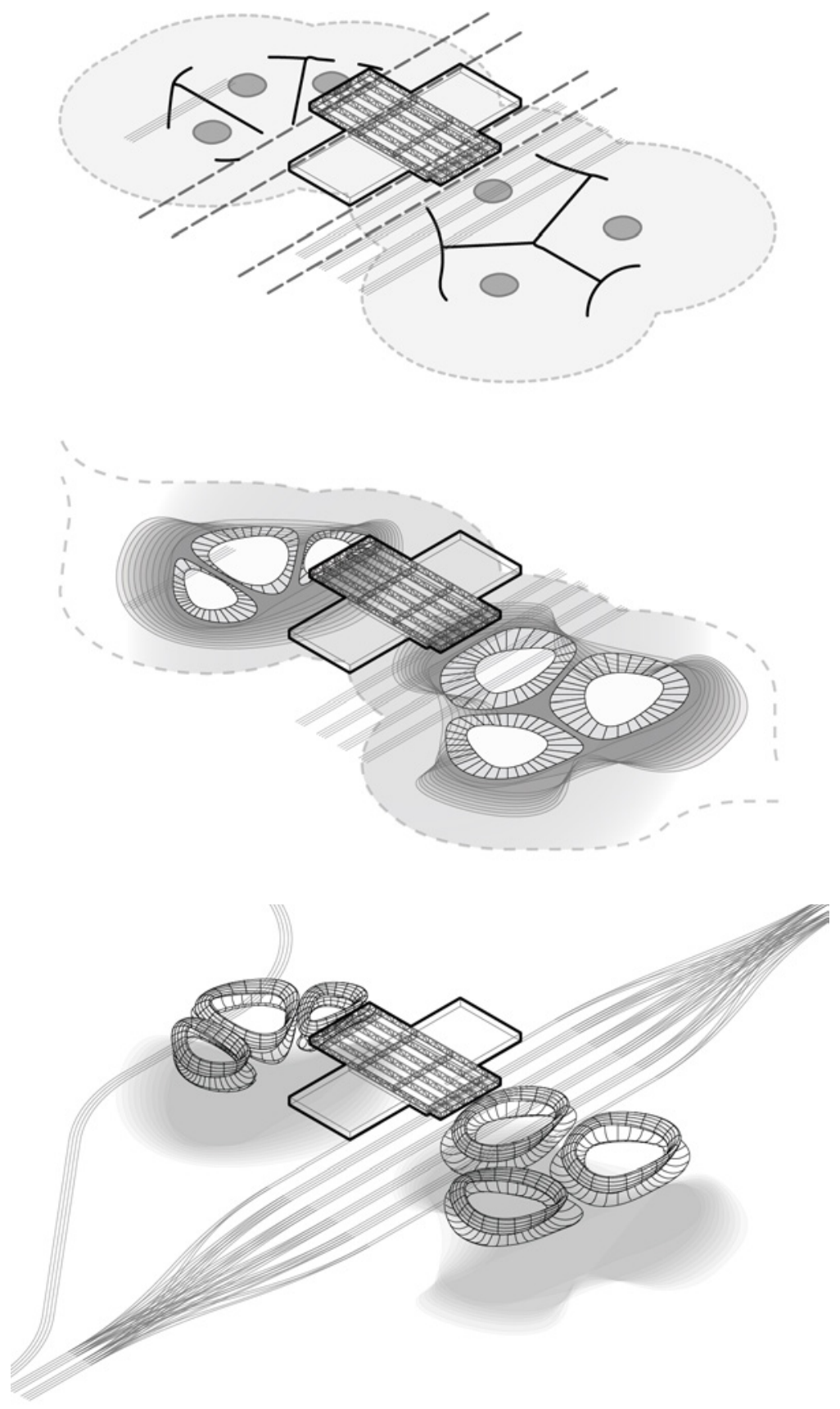
4) Separation of node districts
5) Offset toward central interiors, with a
localized erosion to individual extents.
6) Mobius strip compression rings to
dictate natural lighting paths inside. 
Chapter 5
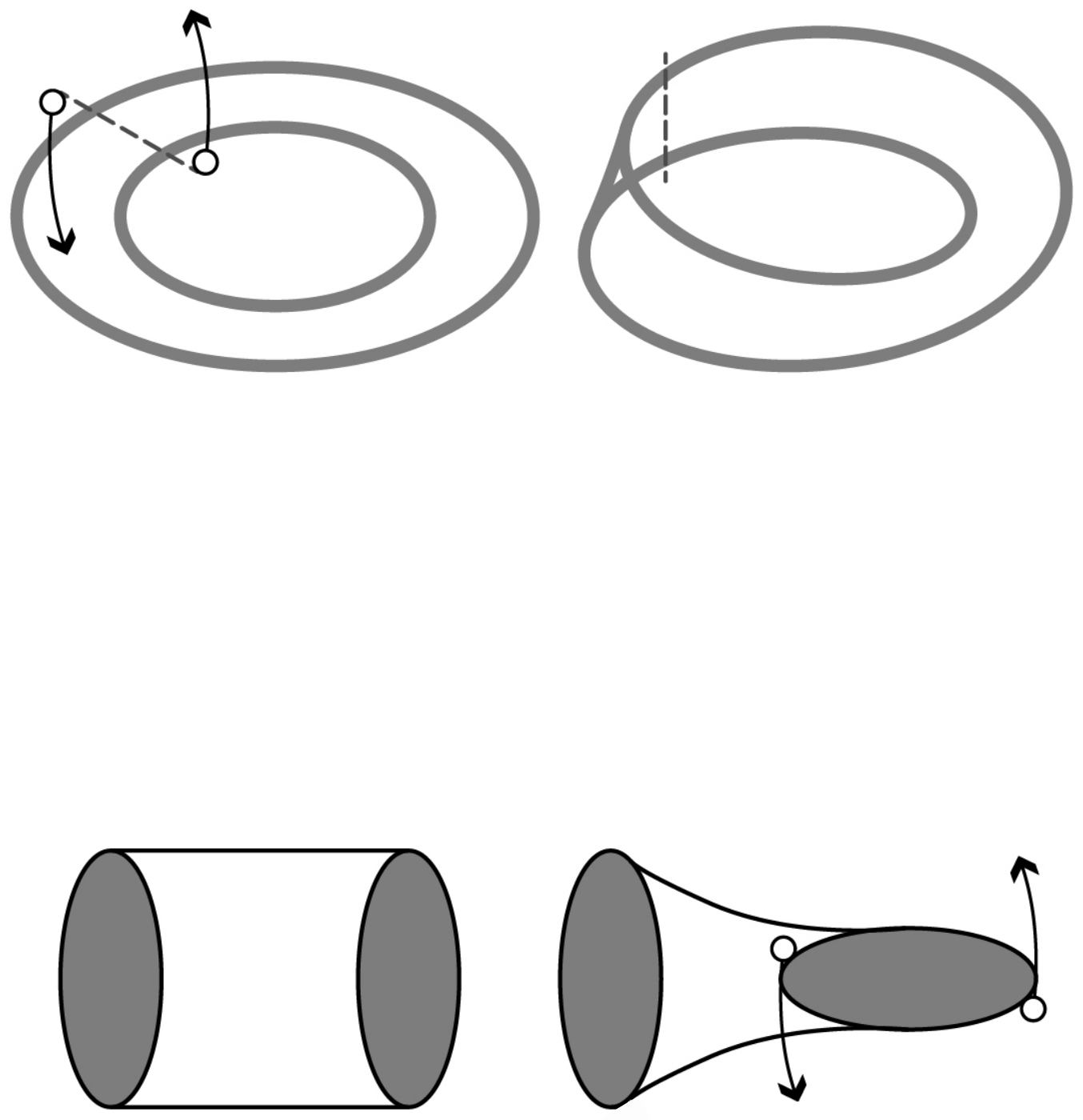

1) Mobius geometry transformation

2) Ovalized torus section vs. ovalized

Mobius section

3) Rotating compression ring profiles,

controlled by parametric sliding values.

(No rotation, 270 deg, 90 deg.)

$-138-$ 

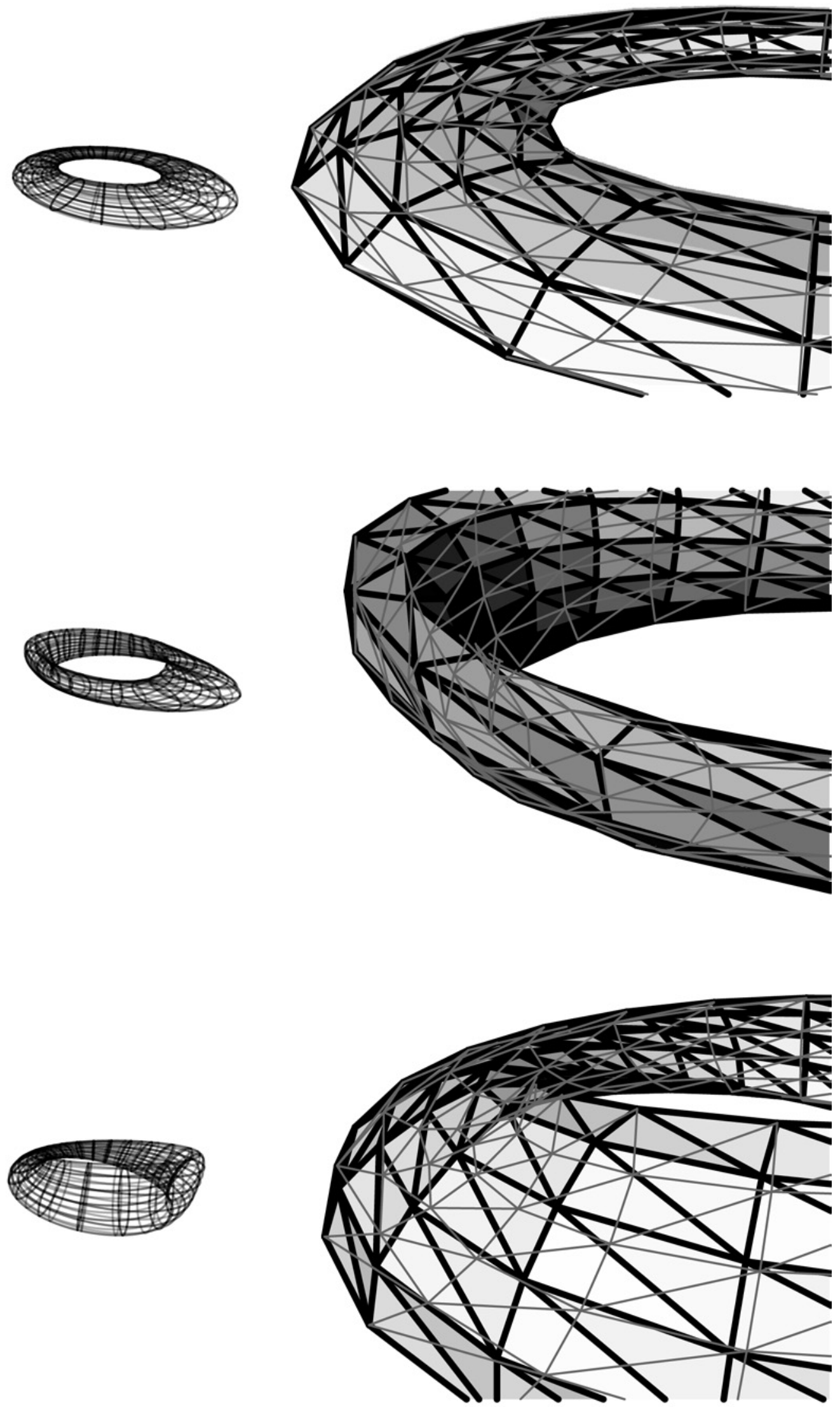

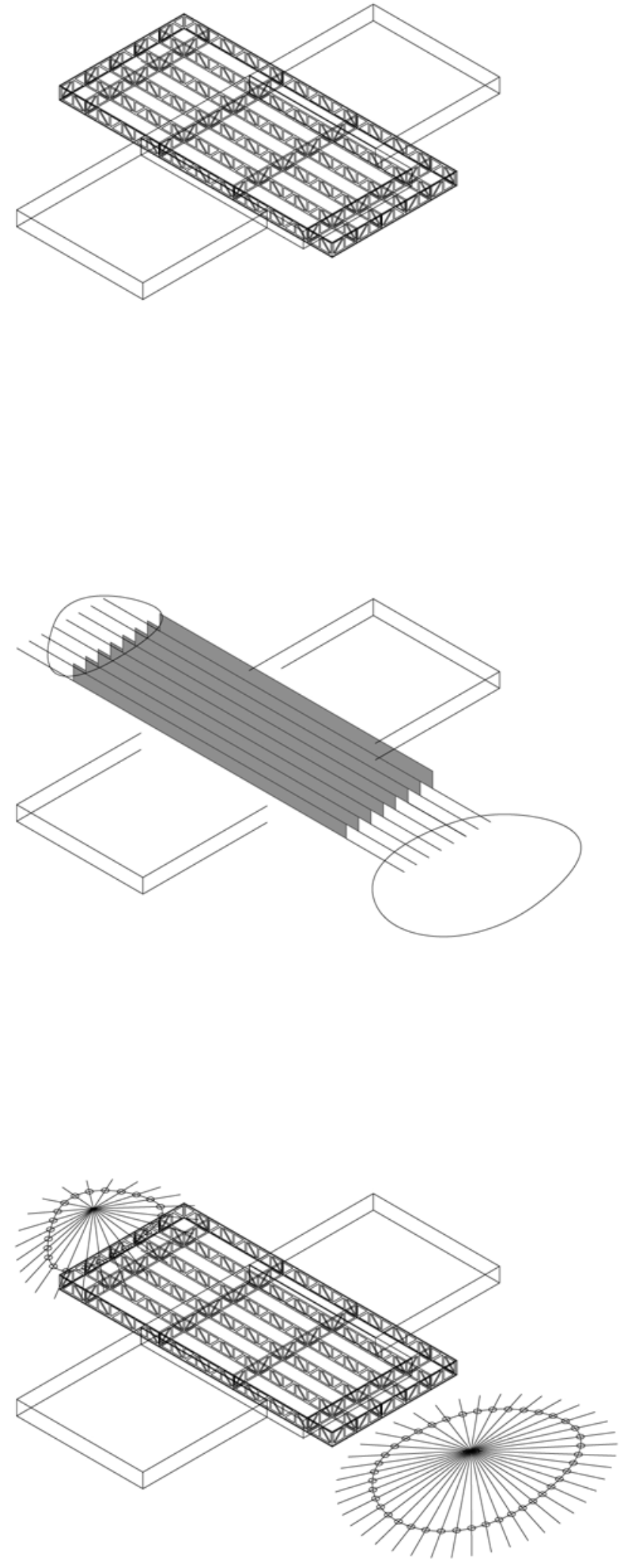

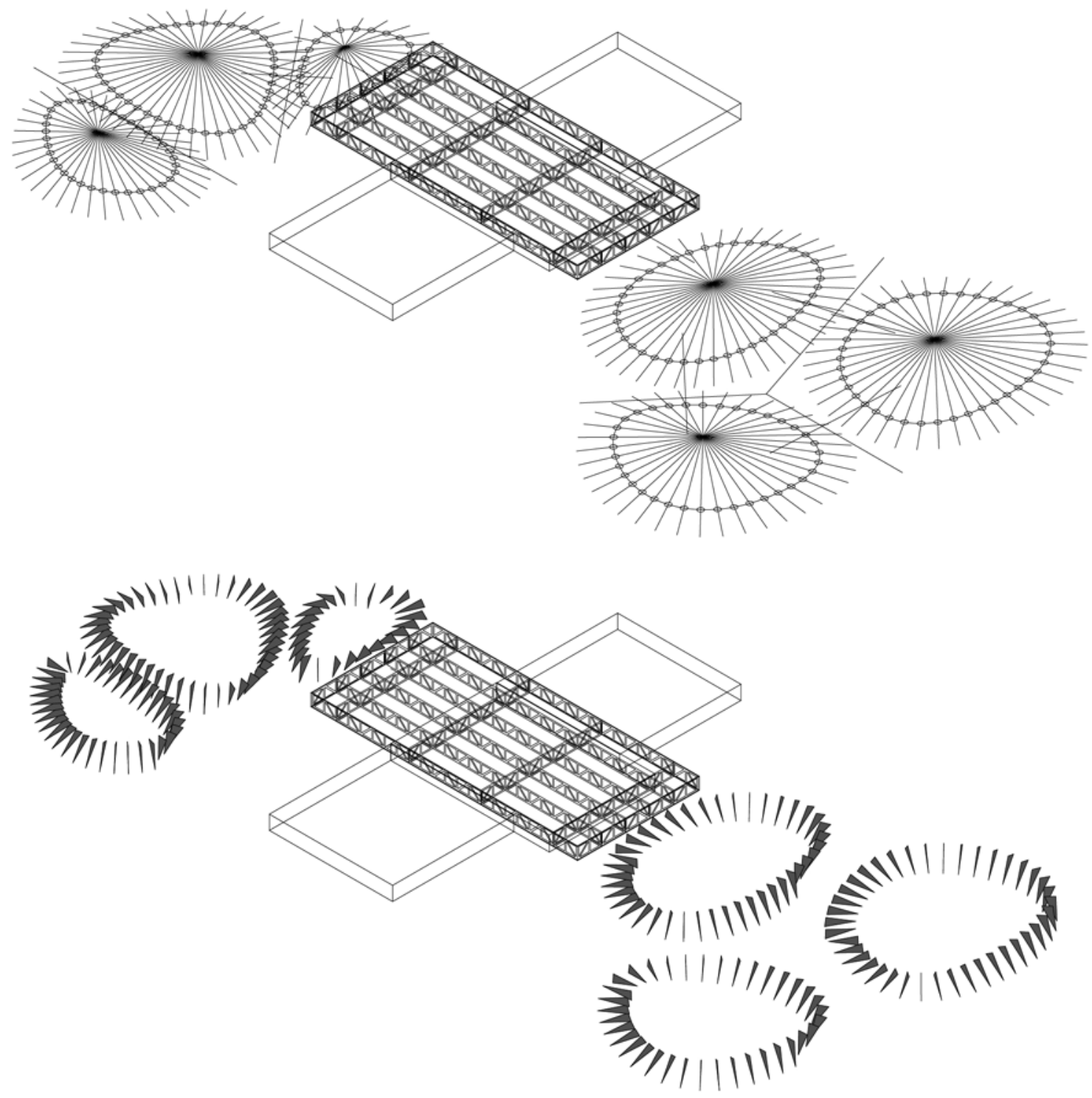

The Mobius Structure can be

extrapolated from the original structure of the Ottawa Train Station by finding the closest point from the existing structural frames to the new ring profile, subdividing the rings by that alignment, and creating the frames based on the parametric profile. 

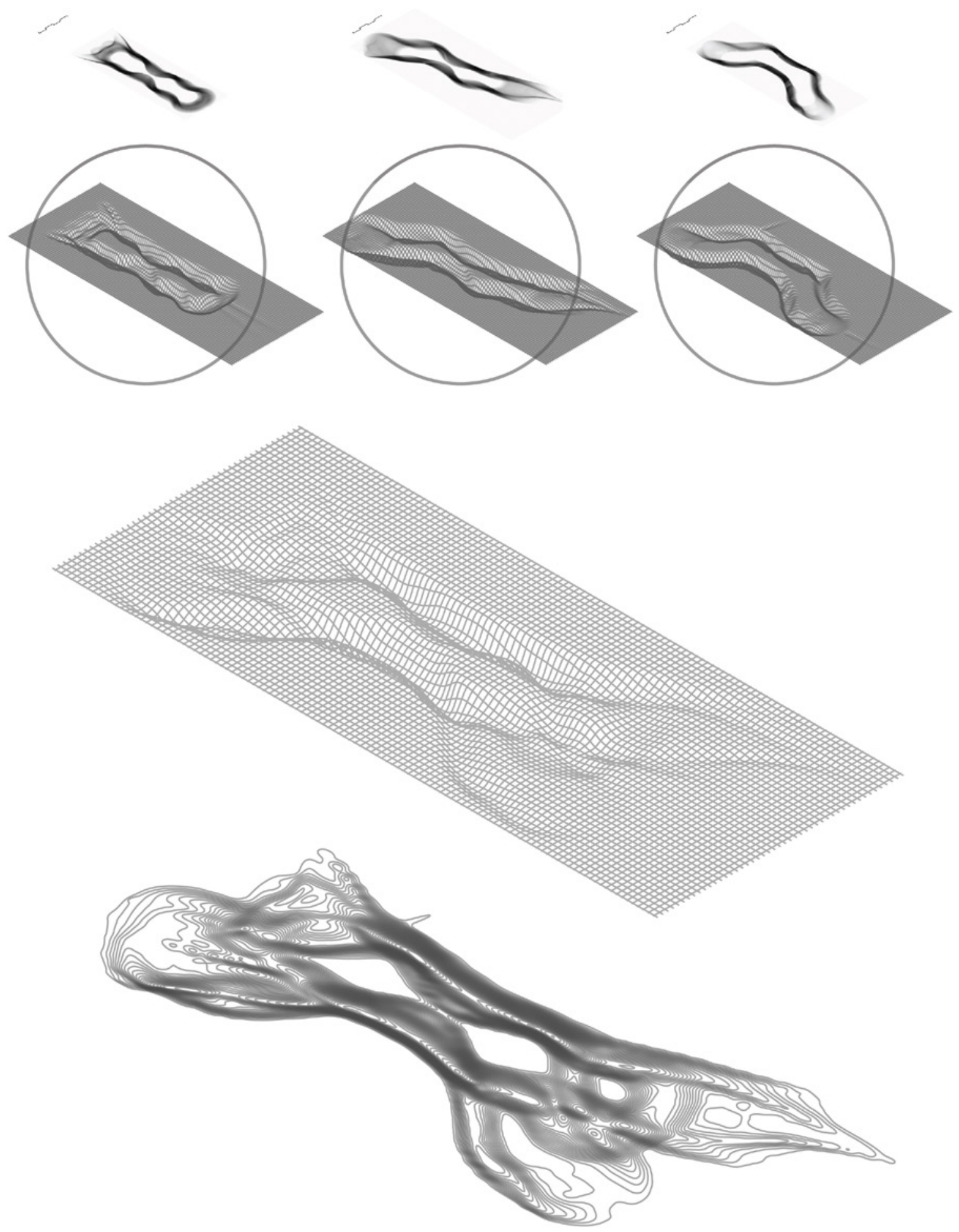

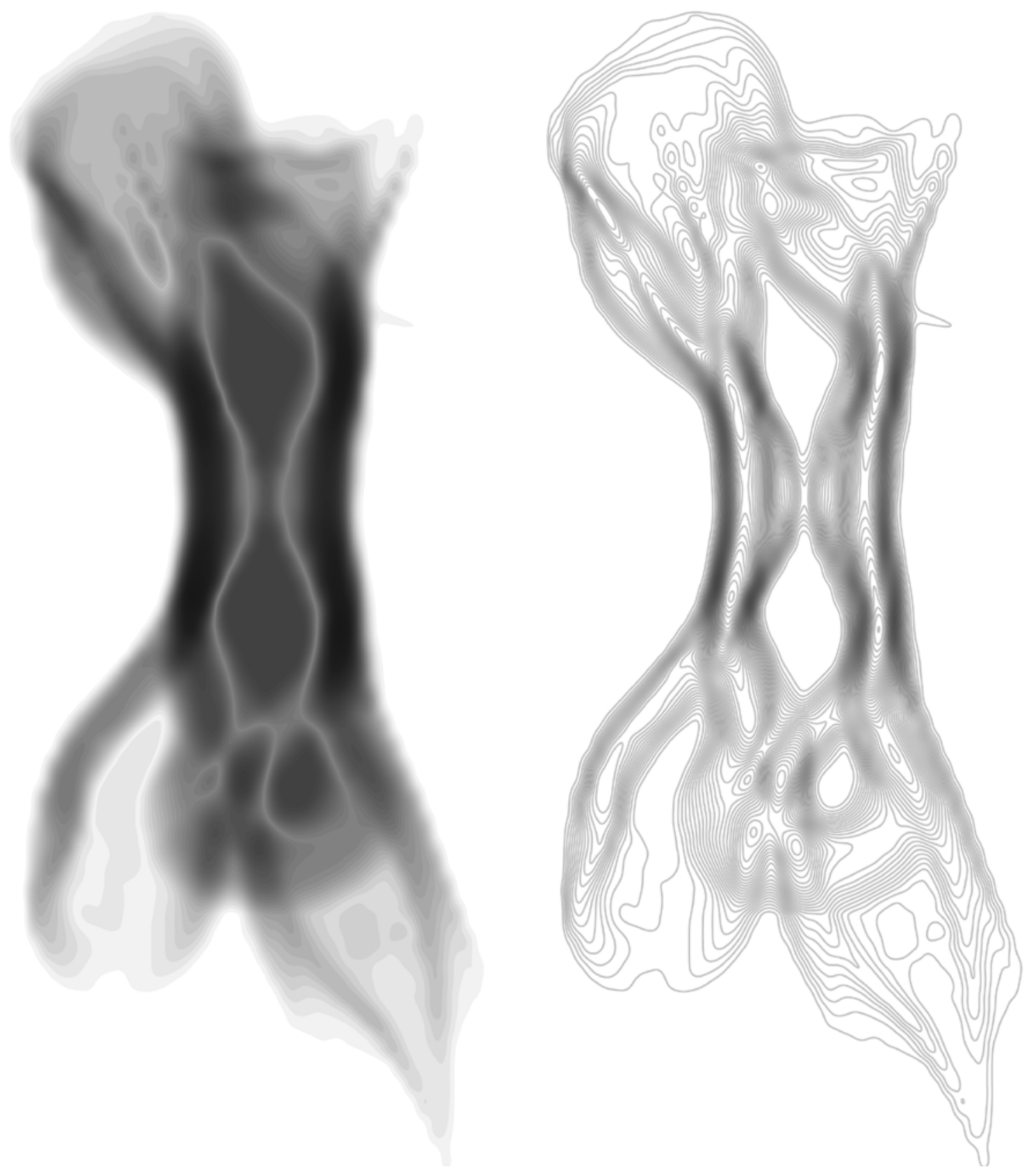

The erosion simulations can be superimposed to create a broader site condition. Using a Grasshopper image sampler, the resonances can be extruded to a 3D surface and define a continuous massing condition. 


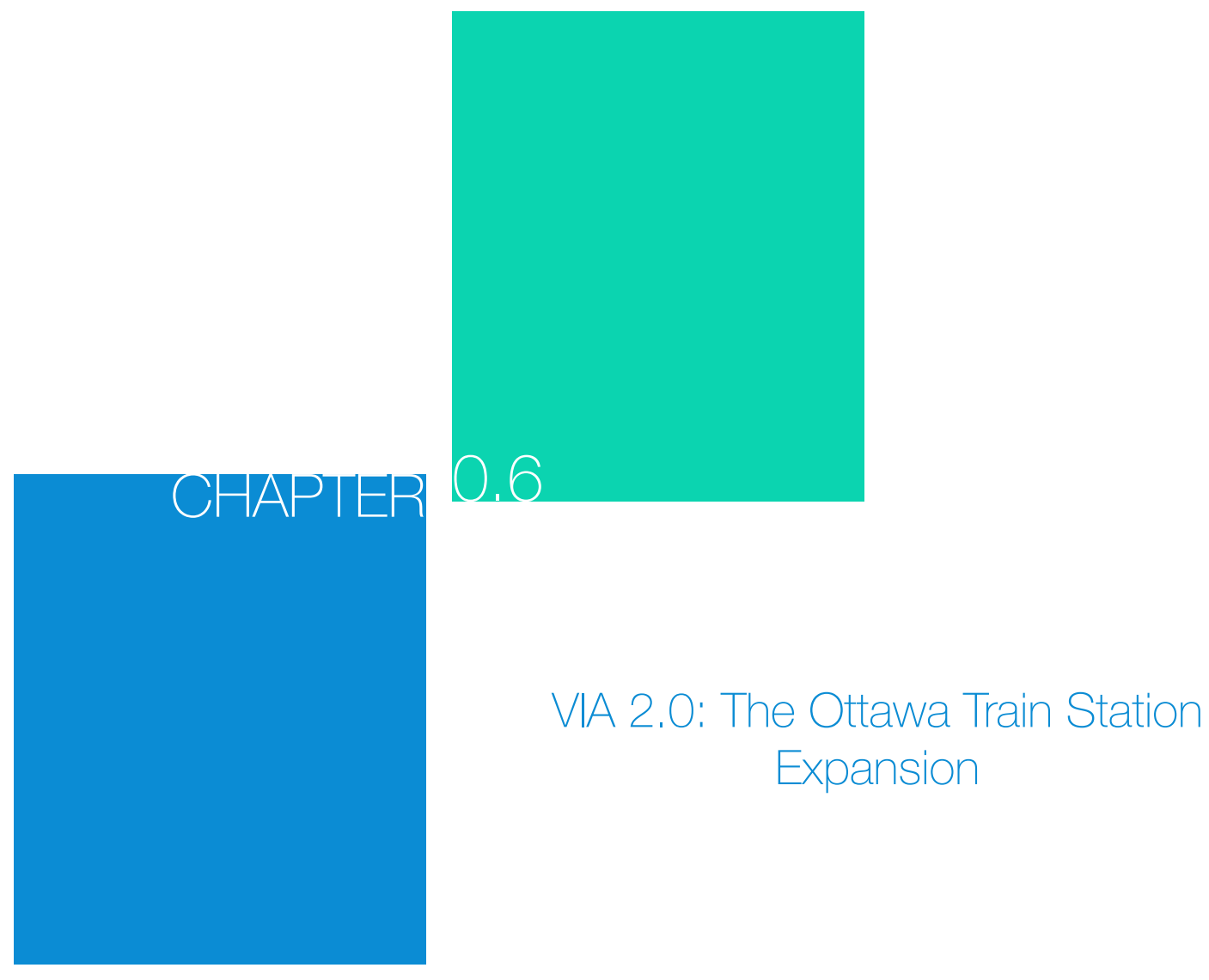


"Airport and train station have one theme in common: both are theaters of travel." _Leon Whiteson 


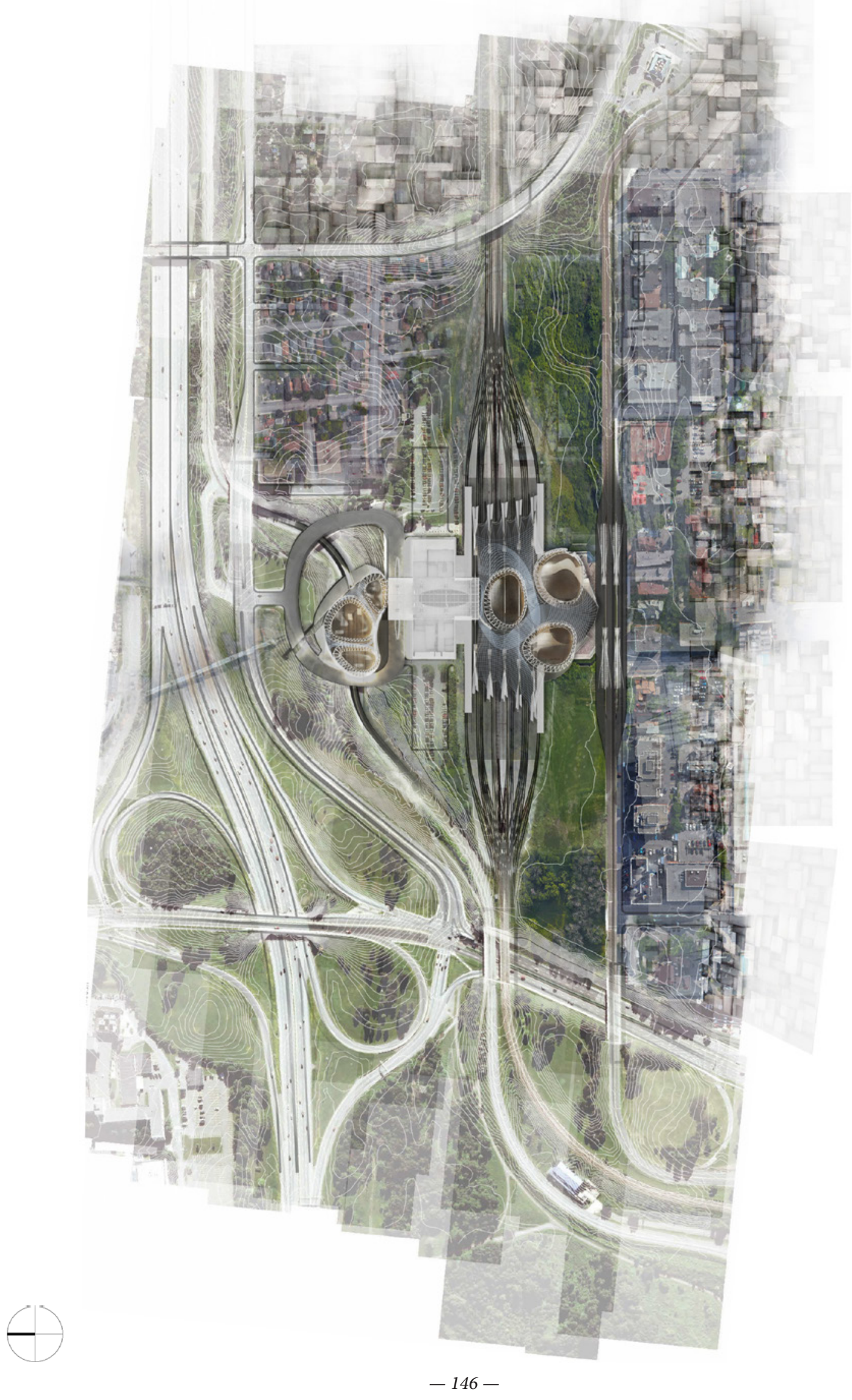

Site Plan 


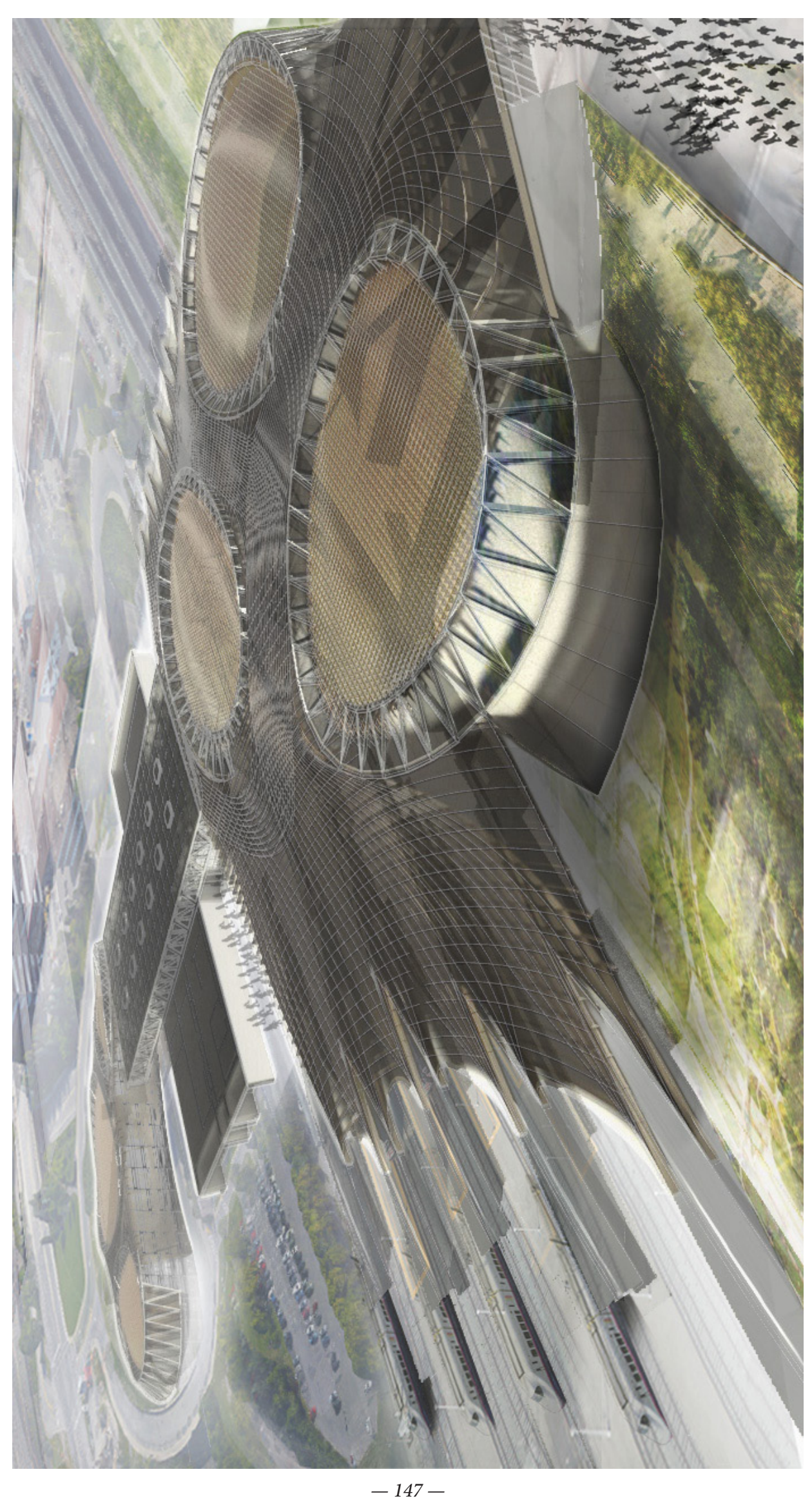

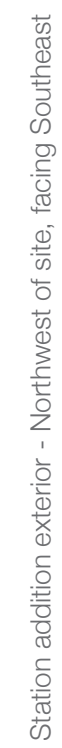




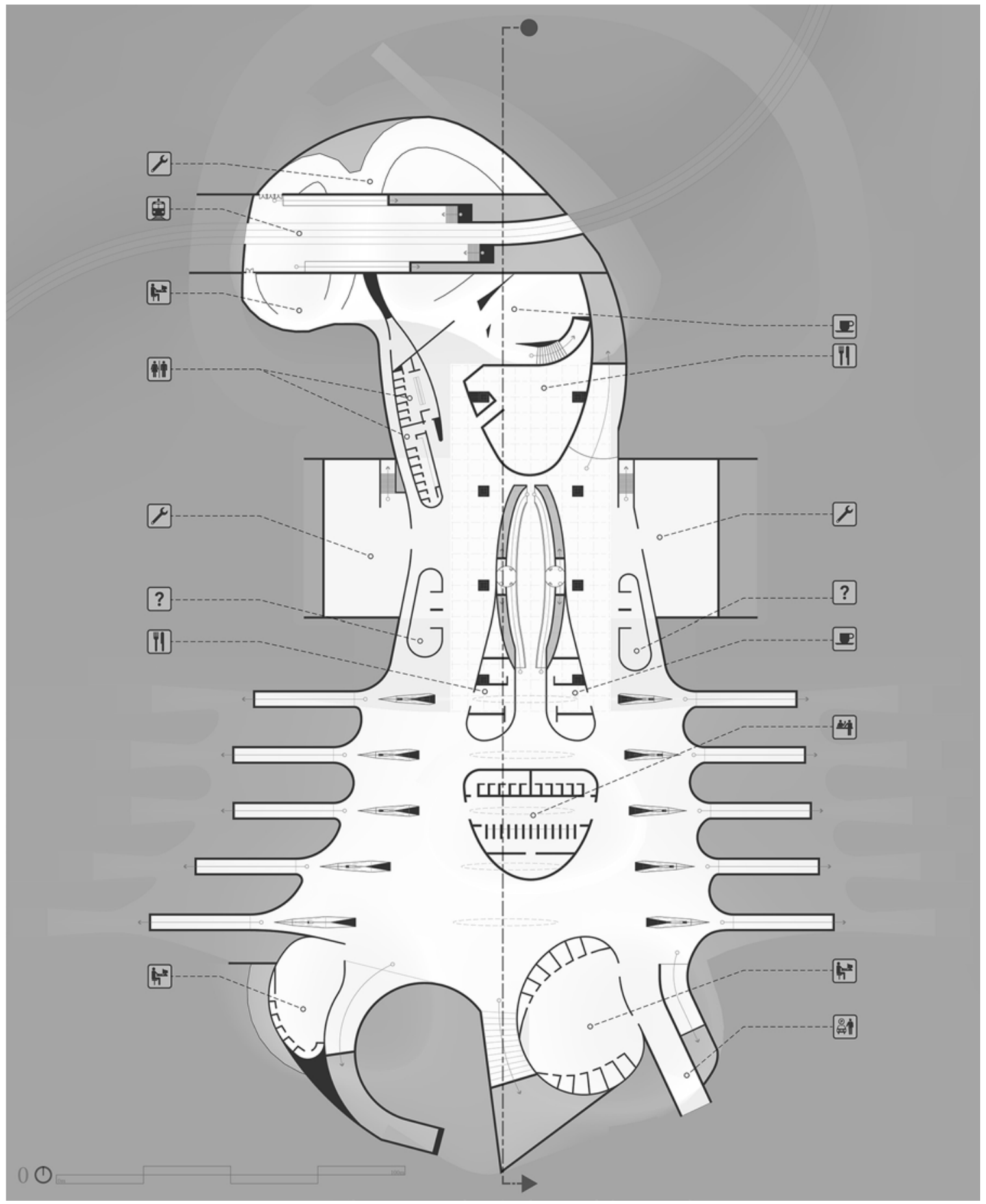




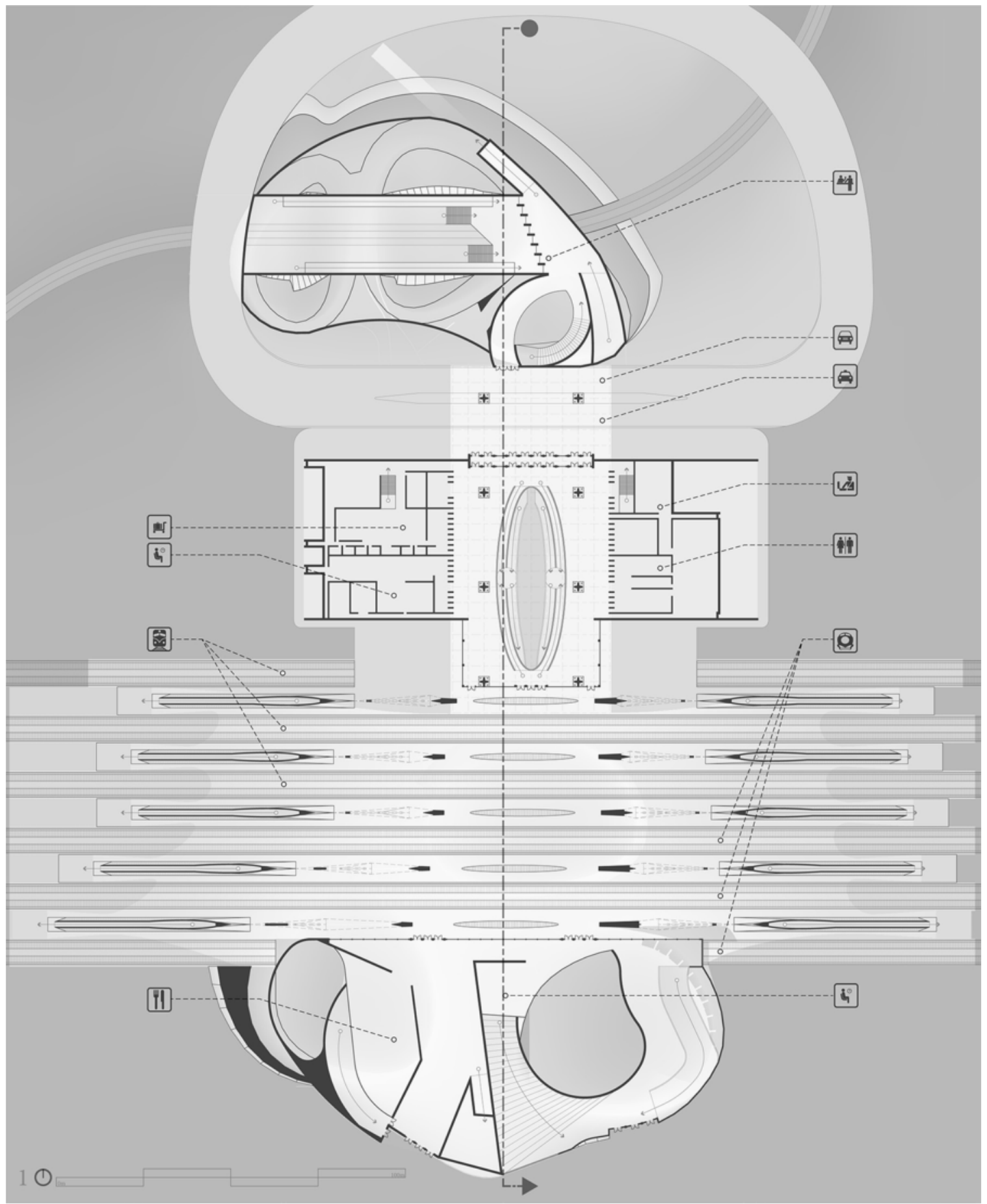




$$
\begin{aligned}
& \infty \\
& 08 \\
& 08
\end{aligned}
$$



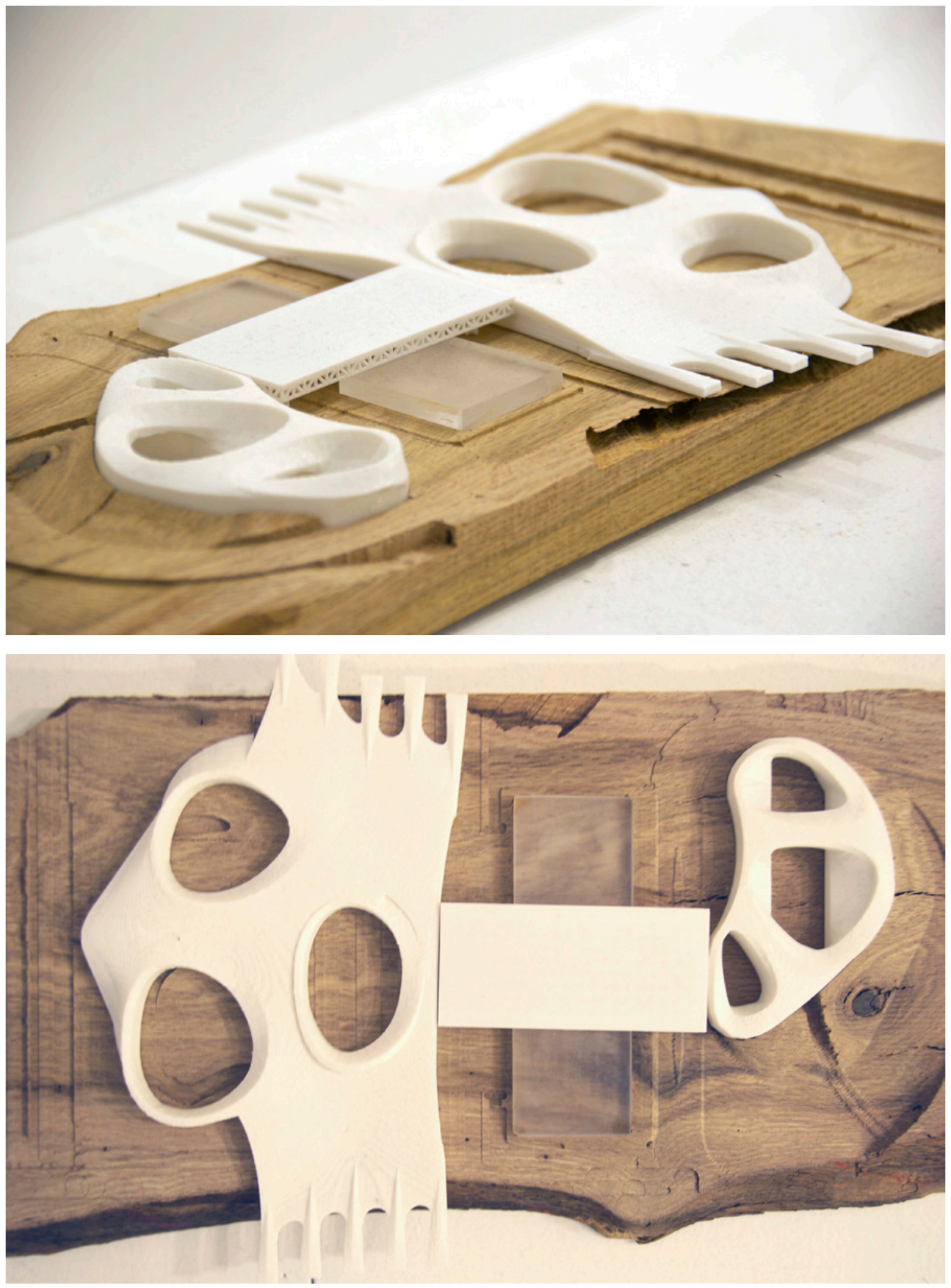


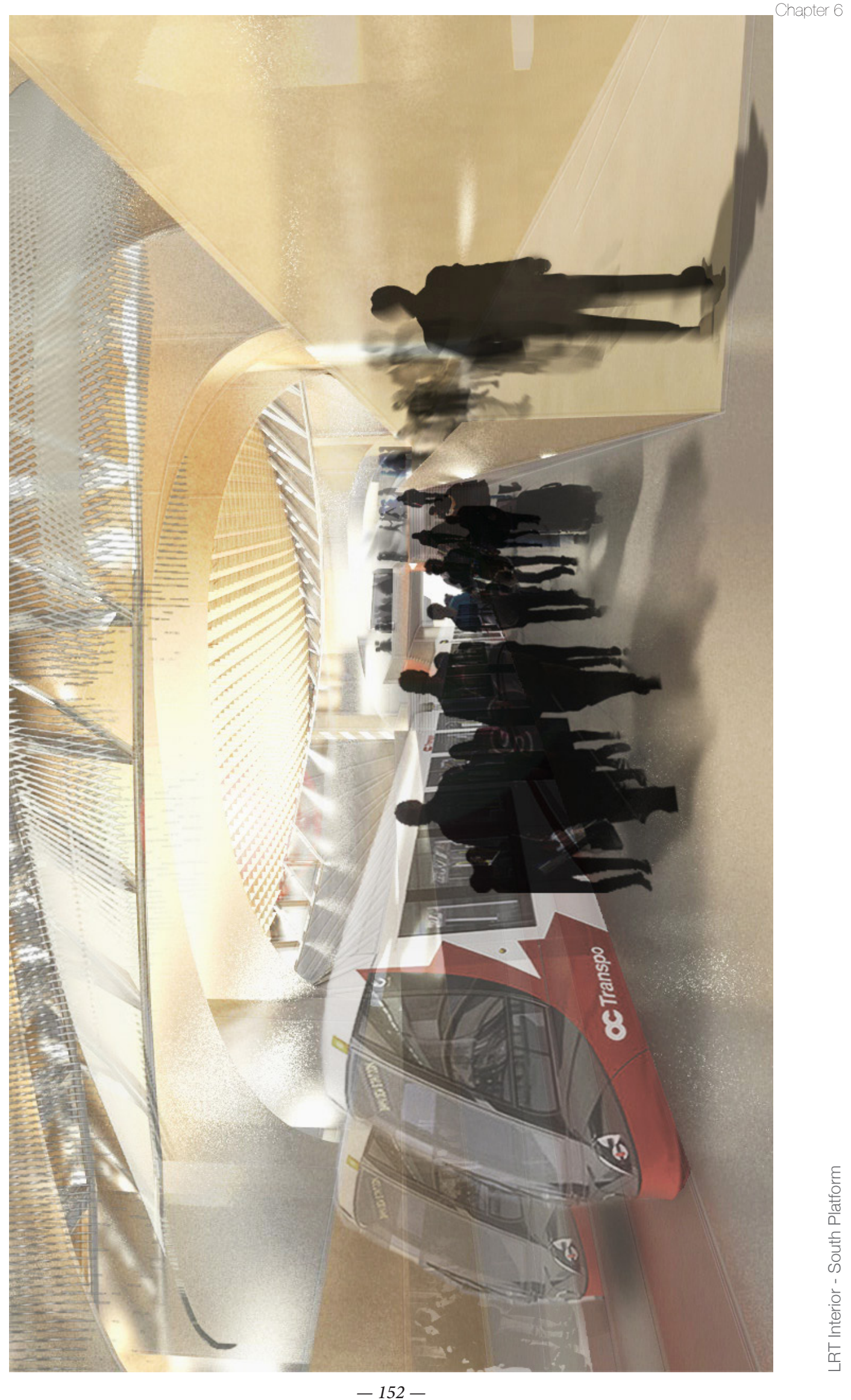




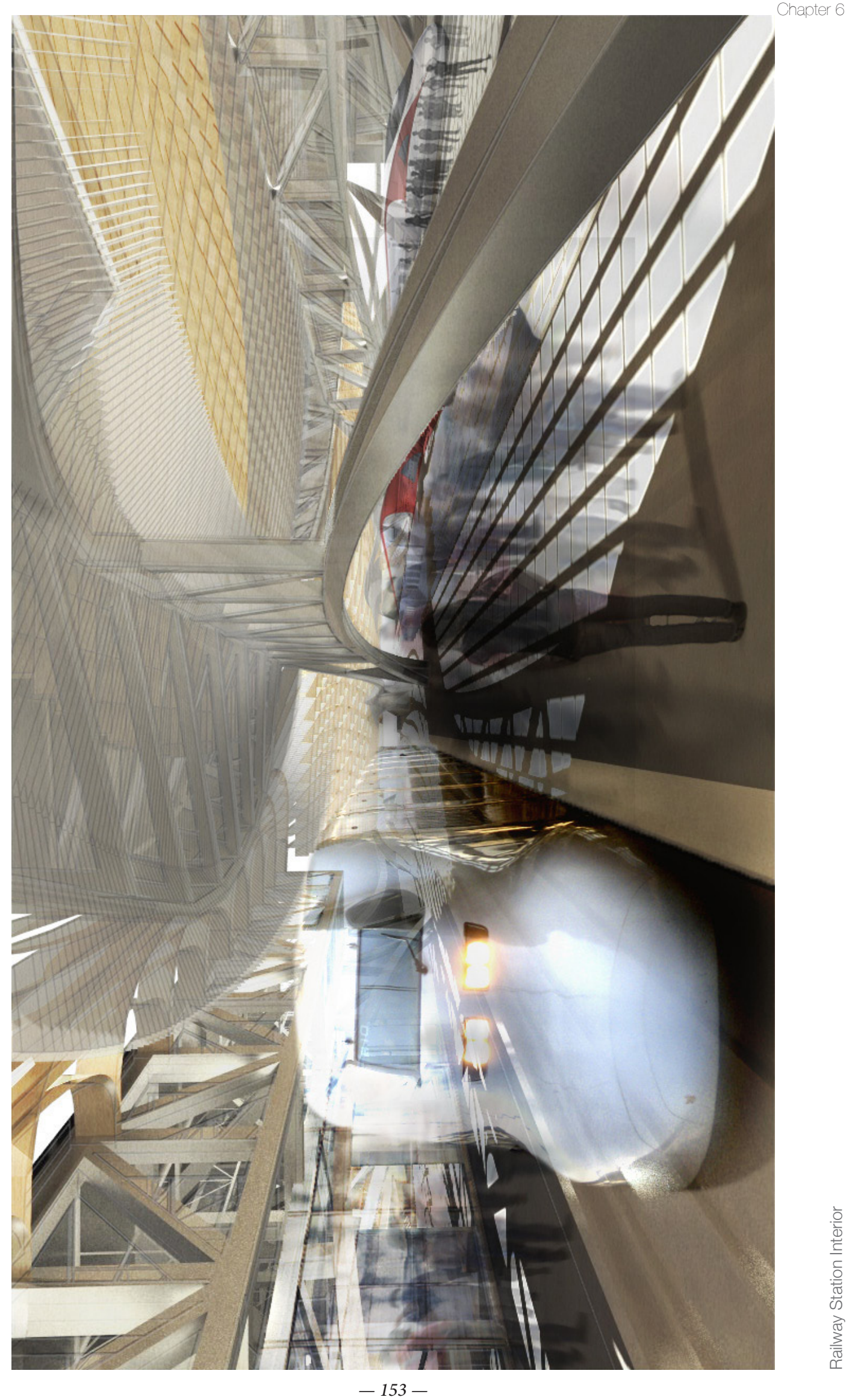




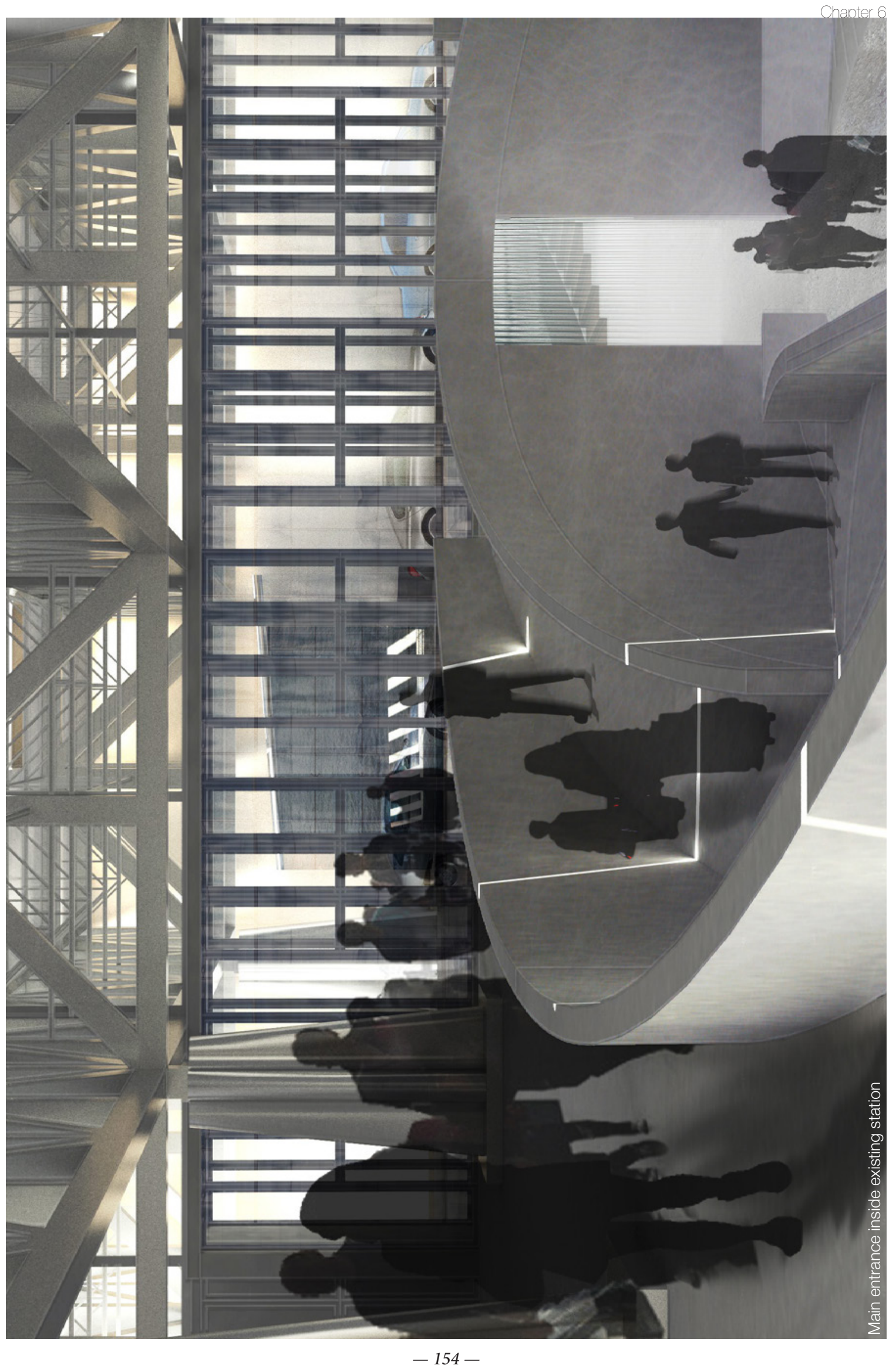




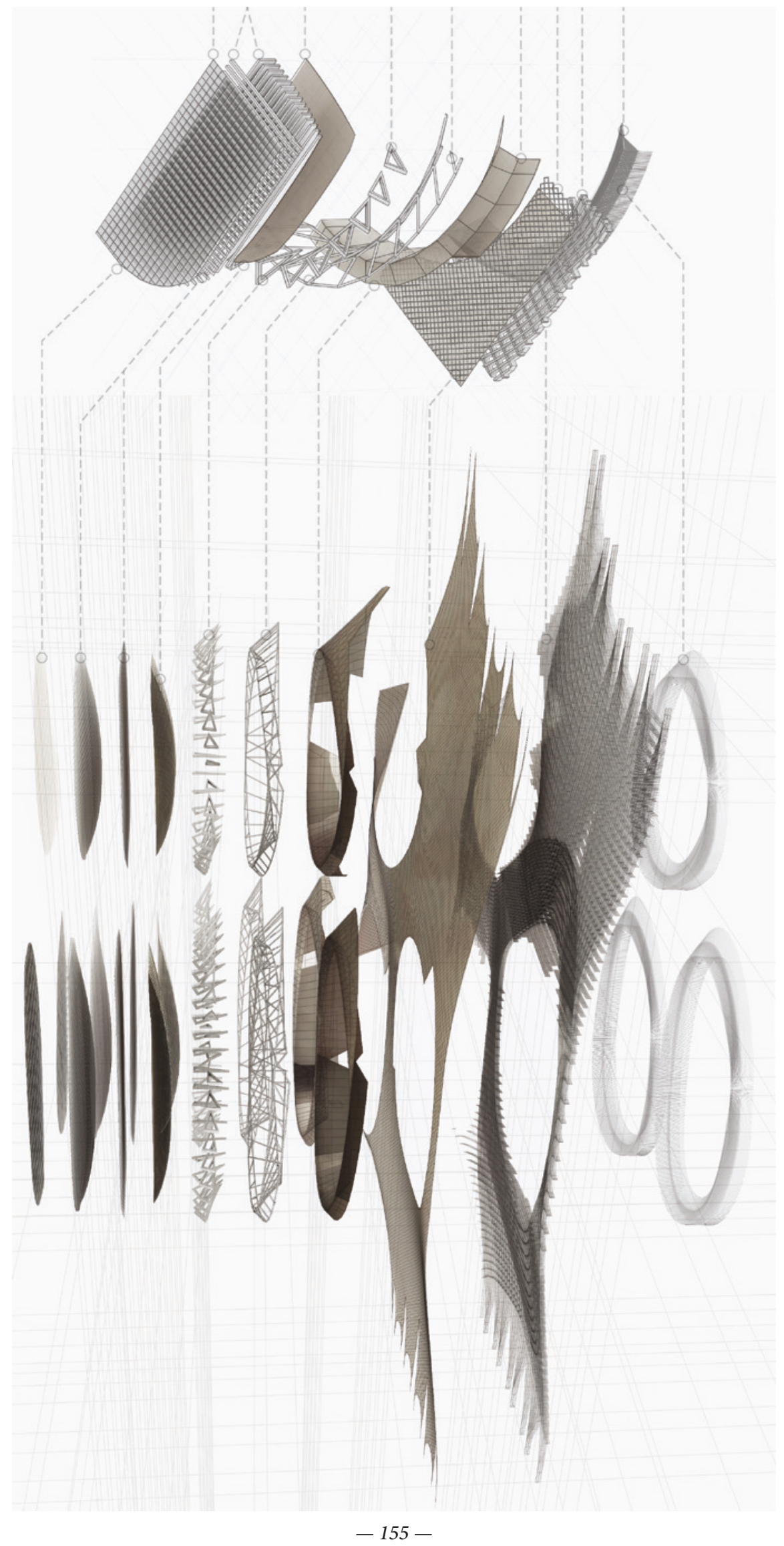

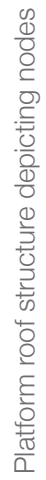




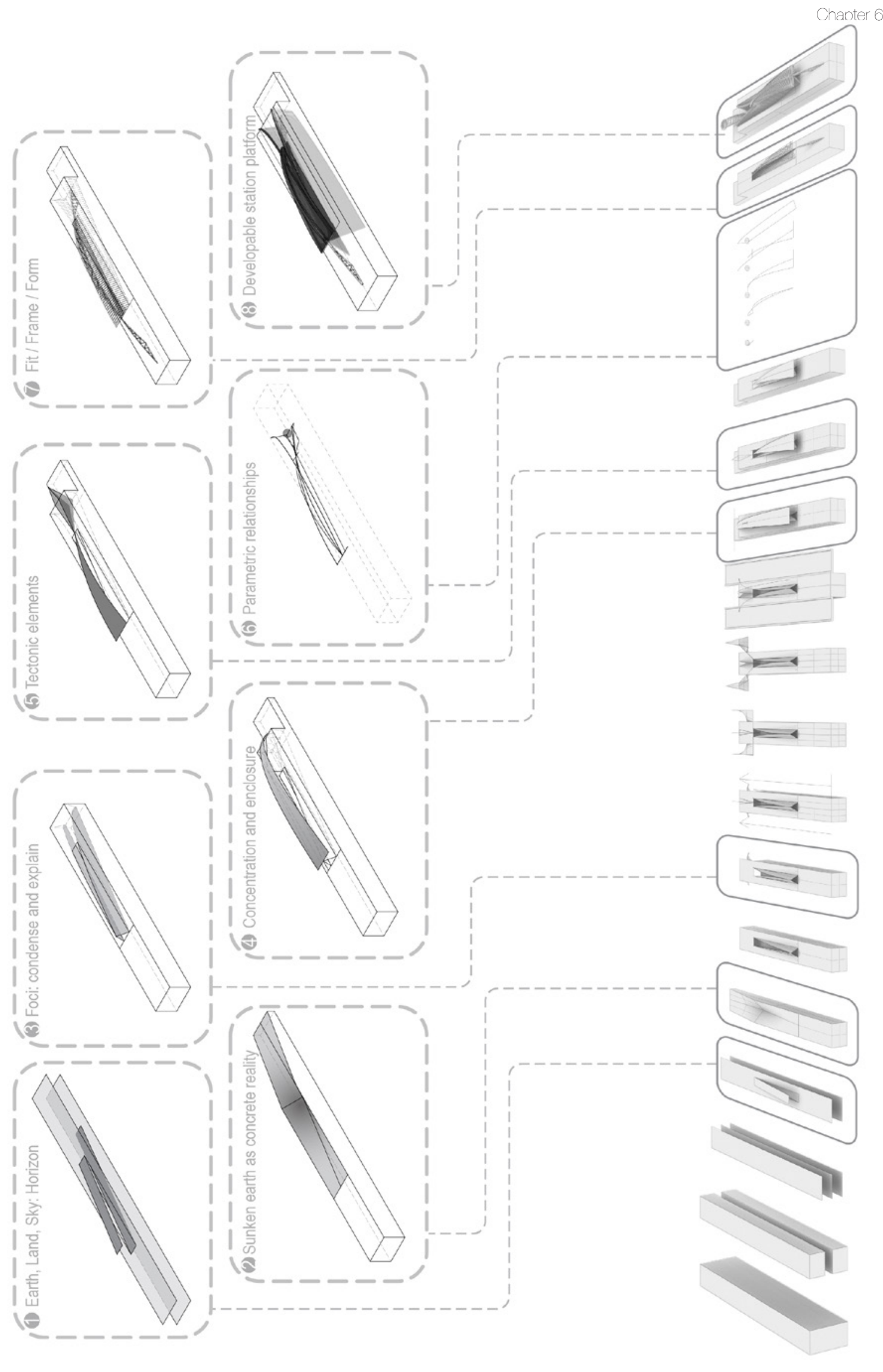




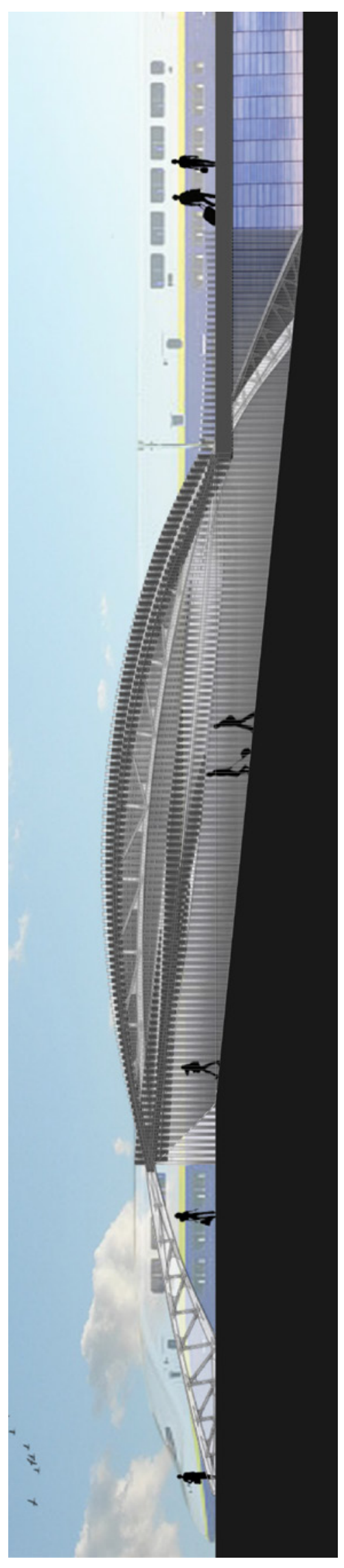

Chapter 6

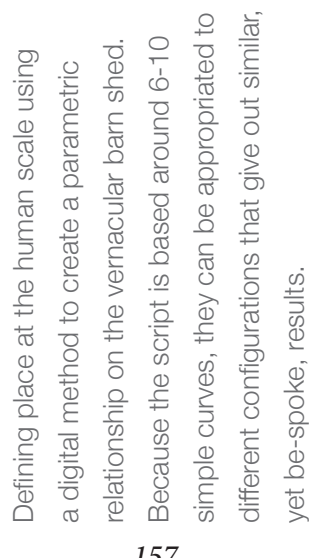



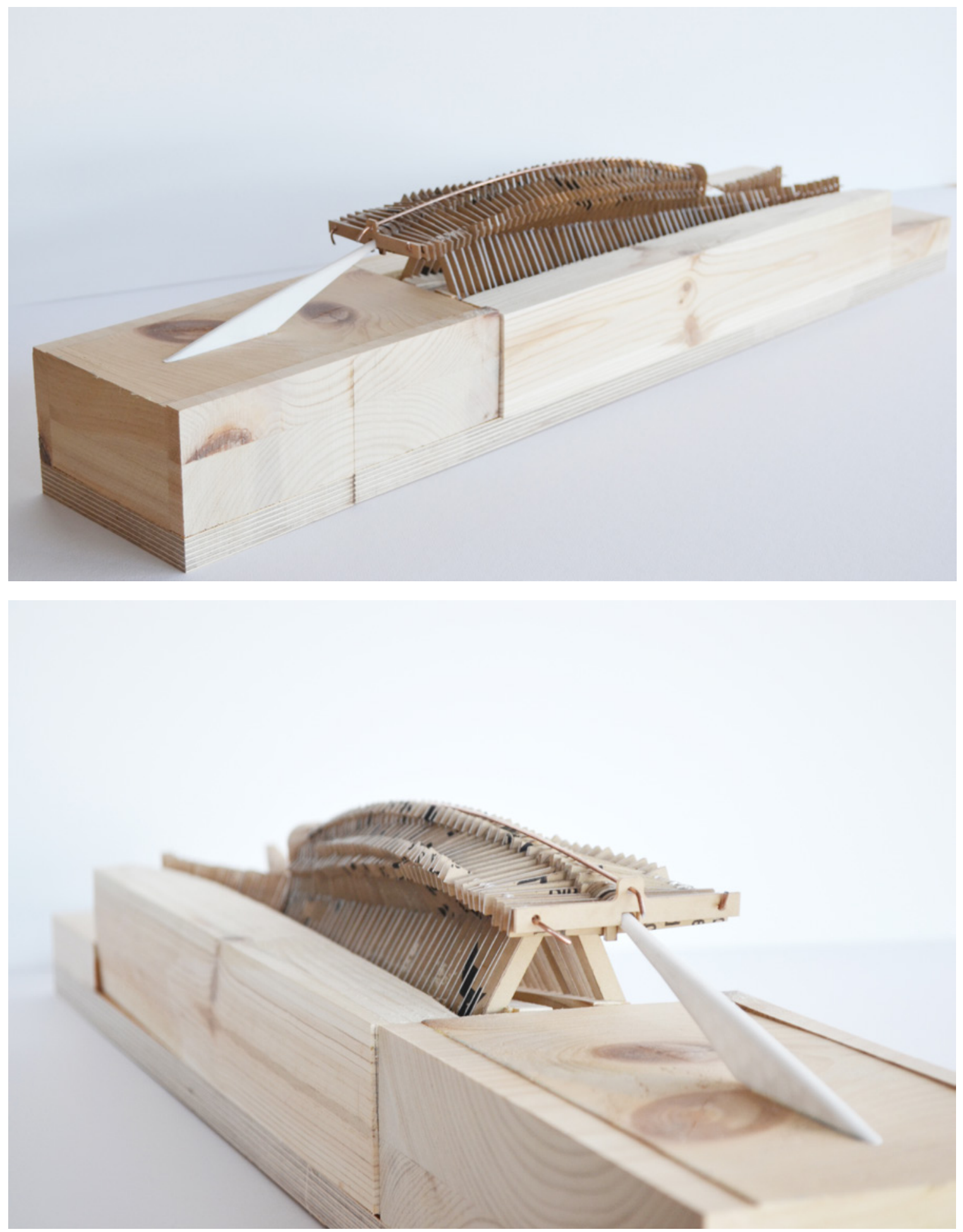


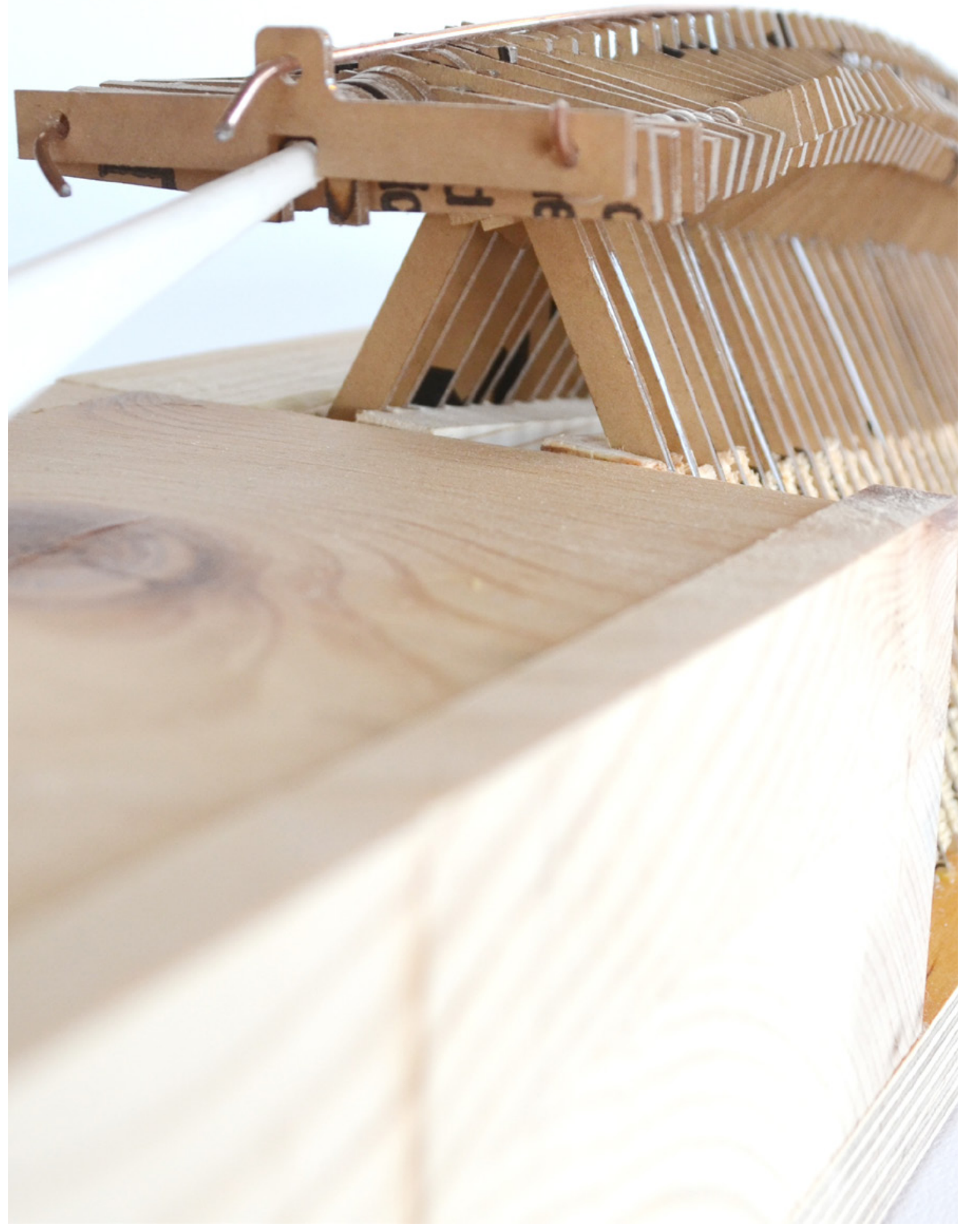



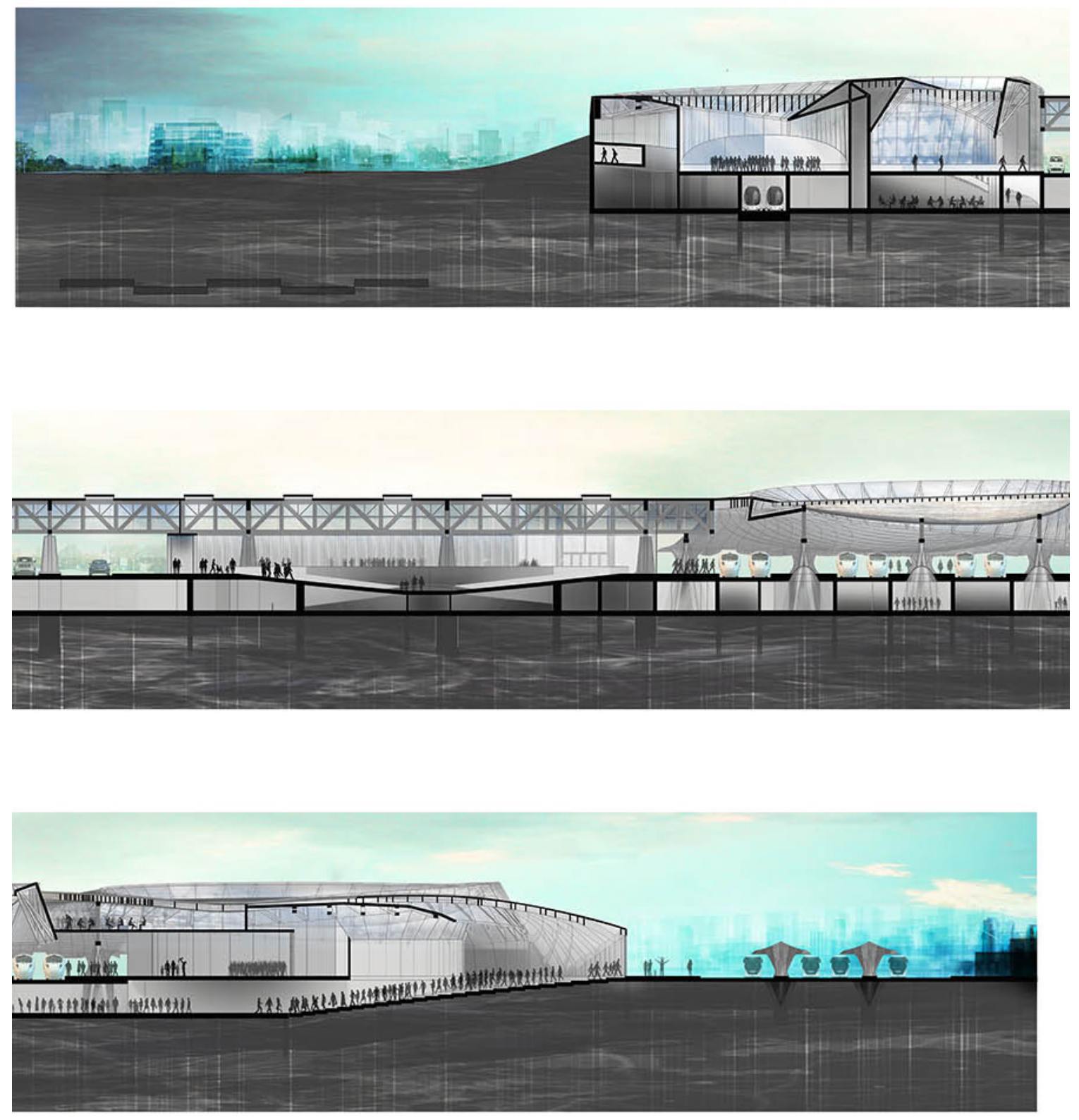

Master Section (hybrid, broken into three parts). 


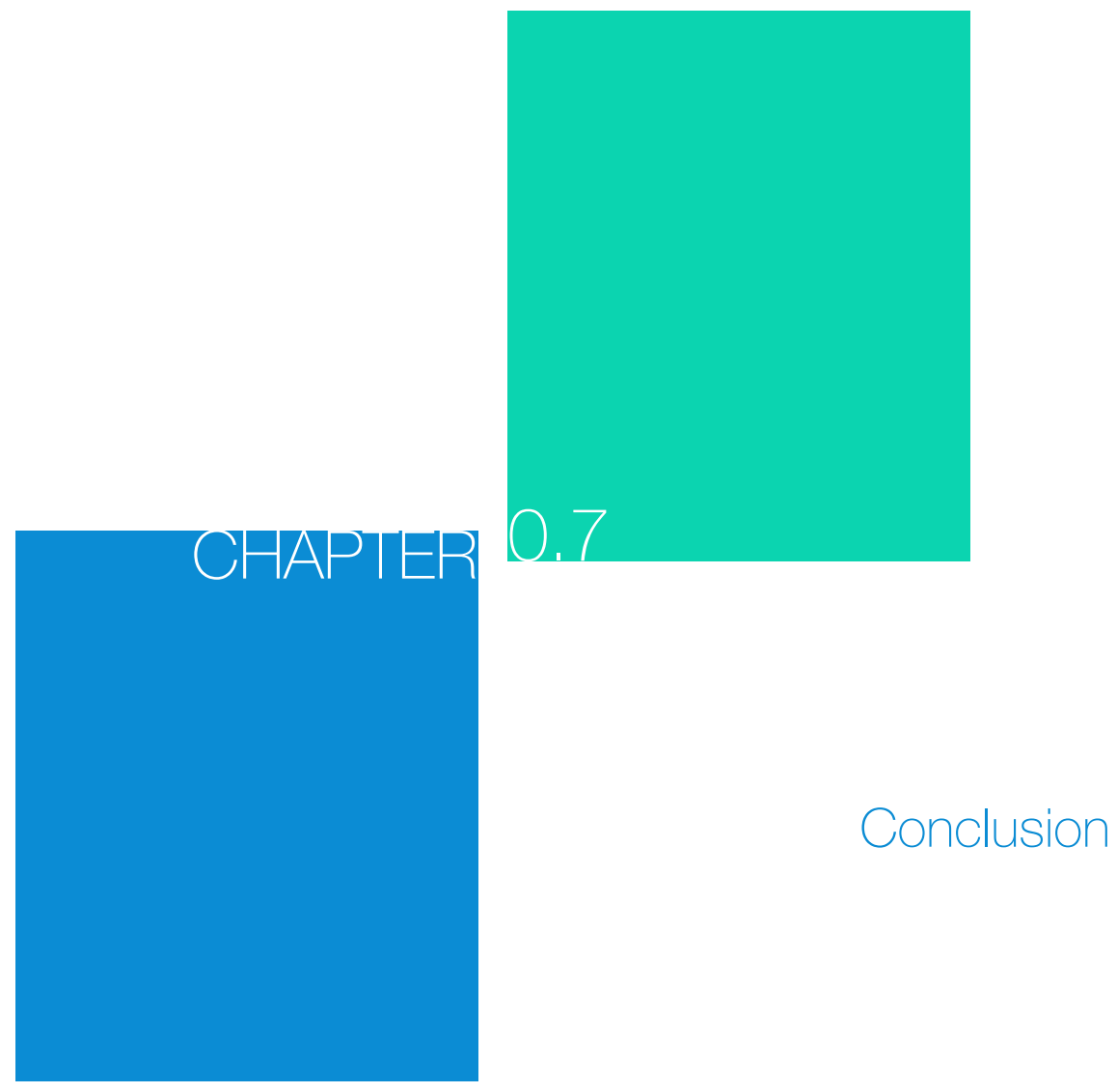


“'-Ism's,' in my opinion, are not good. A person should not believe in an '-ism,' he should believe in himself." _ Ferris Bueller [Ferris Bueller's Day Off] 


\section{$7.1 \quad$ CONCLUSION}

This study set out to explore the notion of regional identity in an increasingly globalizing contemporary architectural landscape, particularly as it pertained to the overlap between vernacular material craft and emergent digital techniques.

The investigation identifies a disconnect between perceptions of physicality and digitality, methods for contextualizing digital architecture in place-form, and bridging the gap between responsive vernacular and computational logics. In tandem, through an iterative process it defines modes for recreating intangible physical objects in digital space, interpolating existing physical traits to produce complete phenomena that would otherwise remain hidden, and determine ways of predicting their generative properties for further extrapolation. If architectural 'Phenomenology' is the study of architectural experience and its sensory properties, 'Phenomena' in this sense is acknowledged as an observed physical process that is acknowledged to exist around us, but is difficult to explain at a definitive level - peripheral sensory evidence. The process of physical deconstruction in the study therefore became integral because it was the one observation that could be considered 'definitive': it records what is there to see. The curves produced in the digital model are curves expressed through the process of cutting and revealing, but in the untouched tree in the forest one would be unable to see (and therefore interpret) the grain. 
Through the physical act of cutting the edge is revealed,

and through the digital interpolation the potential field of grain/fiber is defined. This is how the digital can augment the hidden phenomena: that which we see to be true, but not what we understand to be true.

In recreating these phenomena in digital space, their effect can be produced and reproduced by breaking down their constituent parts and processes using parametric, generative and computational tools. Once these are identified, they can be reinterpreted to broader architectural applications in much the same way as the physical forces on localized natural growths. For this thesis, it is interpreted as a structured field that informs the sinuous movement of mass traffic through the station between the nodal transportation hierarchies, grafted to the original 1960's station. The spatial results of this information are interpreted architecturally to subtly guide these movements through the station by incorporating them into the design process. In doing so, the proposal, and particularly the roof, become a strain of 'meta-wood' that transcends the sentimental kitsch of the vernacular. It is instead a roof of regional phenomena that has been applied to create a meta-wood system, where it engages in dialogue with the divergent and emergent field lines. As a meta-wood system depicting regional independence, 'place' is created within the physicality of the wood phenomena.

The key issue that came up during the course of this research was establishing, reinforcing, and ultimately safeguarding the integrity of the connection between the digital models and the physical objects. The flexibility in 
the parametric software became integral for finding the associations in the material investigation, which produced successful results (especially with a dearth of relevant case studies). However, this presented a paradox: at what point should the designer go from the investigative metaphor to the physical architectural implications? Through the course of the study, for an extended period of time it seemed that this was on the verge of presenting itself - the moment of enlightenment. This came instead through the broader interpretation of the various results produced from the study. No silver bullet ever emerged from the fog; rather the gray investigative area became much richer than the black-and-white expectation.

Future research holds promise to further refine how a Digital Regionalism might unfold. An expanded body of knowledge on the way localized phenomena acts and how the patterns can be more reliably interpreted and differentiated from one another, in terms of localization, would improve the regional distinctions for a Digital Regionalism. Much of the research revolved around the dissection of wood growth phenomena using digital modeling tools. This choice was made because the scope of the study required focusing on an object that could be reliably recorded and extrapolated to develop the project. Deeper separation between particular tree growth types occurring in specific regions could give deeper meaning to the study - for instance, differing growths from the Boreal throughout the Canadian Shield, to the rain forests on the West Coast, to the Atlantic Maritime ecozones.

The context of Ottawa was chosen for this thesis 
due to previous individual knowledge of the city and

surrounding area, mainly from long-term habitation.

When writing about regional identity, it must come from

the personal, local perspective rather than appropriating

the identity of another region. However, the research

shows promise in how it could be applied to other urban

centers along the $1200 \mathrm{~km}$ Windsor - Quebec City corridor.

In particular, how the parametric definitions created for

this project could be applied to Union Station in Toronto or

La Gare de Montreal should high-speed rail get approval.

The advantage of a parametric space is its malleability -

one can apply a defined process to different contextual

situations. The logical extension of this research then is to

see which parts can be applied to the other scenarios, and

which cannot. This type of continuation would allow for

an even stronger regional architecture that would reinforce

the notion of identity and place across this major urban

Canadian corridor.

With the improved proficiency of computation

to simulate, predict and model phenomena, the localized

conditions could, once again, become a central principle of evolving a new vernacular. This Digital Regionalism would be a fundamental shift from its predecessors, as the singular customization of the craftsman's vernacular hut gives way to the mass customization employed by parametric modeling in the construction of large-scale projects. It offers once again to put place at the forefront of design and locale as a means of expressing independence. By doing so, it promotes a strong, clear identity in the architecture. This is a rejection of the autonomy of digital design, and a 
rejection of the historicism of phenomenology. It is instead

a merging of these critical issues side-by side in developing a new meaningful architecture.

\section{2 $\quad$ POST-SCRIPT}

As a democratic infrastructure, rail stations play an important role in the urban makeup of the 21st century city, requiring a large footprint by virtue of their function. In contrast, the wood knot exists as a small microcosm in the greater structure of the tree, growing from the core system as a pure response to its surroundings.

This architecture has reversed these roles. The train station has grown out of the city's established systems through its relationship to surrounding context; it has become the microcosm of the city. Paradoxically, the material culture of wood in Ottawa has expressed the knot as the macrocosm of space in this project - the traveler within the node. This becomes most apparent in the site plan of the thesis.

As an urban project, this role-reversal indicates that the vernacular (as a traditionally small-scale building methodology) can be simplified to its core principles, which will always remain scaleless. It does not necessarily need to be tied to a form, but rather to an identity and a response. The form is the microcosm of the city; the material is the macro of the wood. Their common characteristic is the field condition in which they operate. This has produced the urban placeform between site, city, 
architecture, and inhabitant. Here, regionality is empirical,

not closed within the aesthetics or academics; and the

byproduct is a rich architectural methodology specific to its

region. This proposal is simply natural infrastructure.

Finally, this placeform (as an urban anchor

connecting its surroundings with a purpose of its own)

produces a defacto Landmark, bringing closure to the

spectrum of Kevin Lynch's city elements on the site into the completion of the building proposal. 


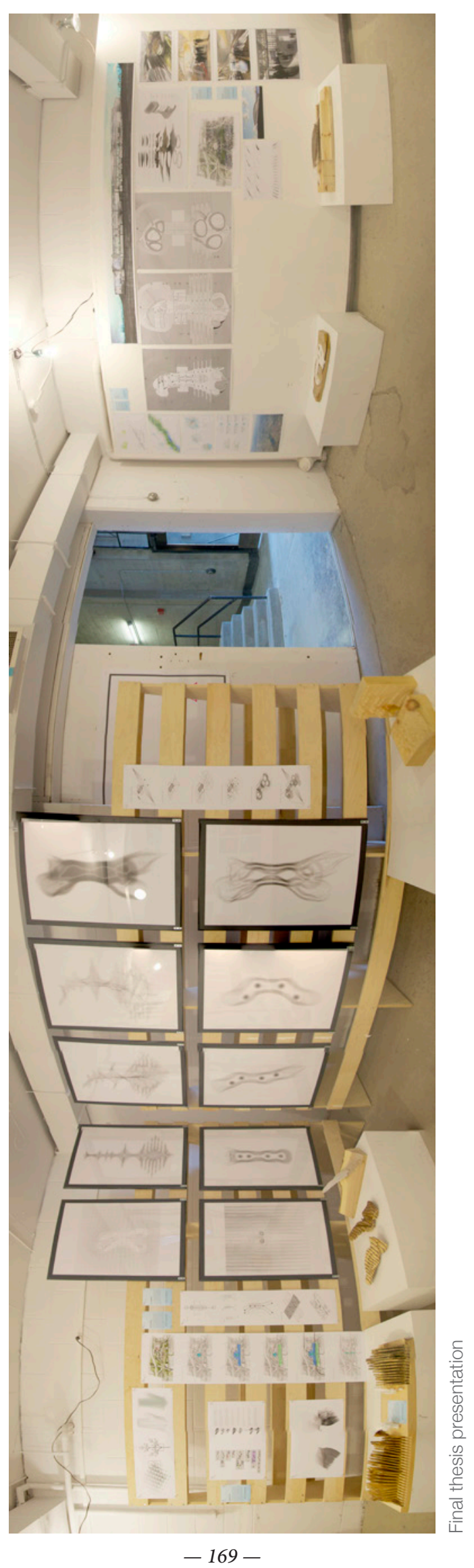

Chapter 7 


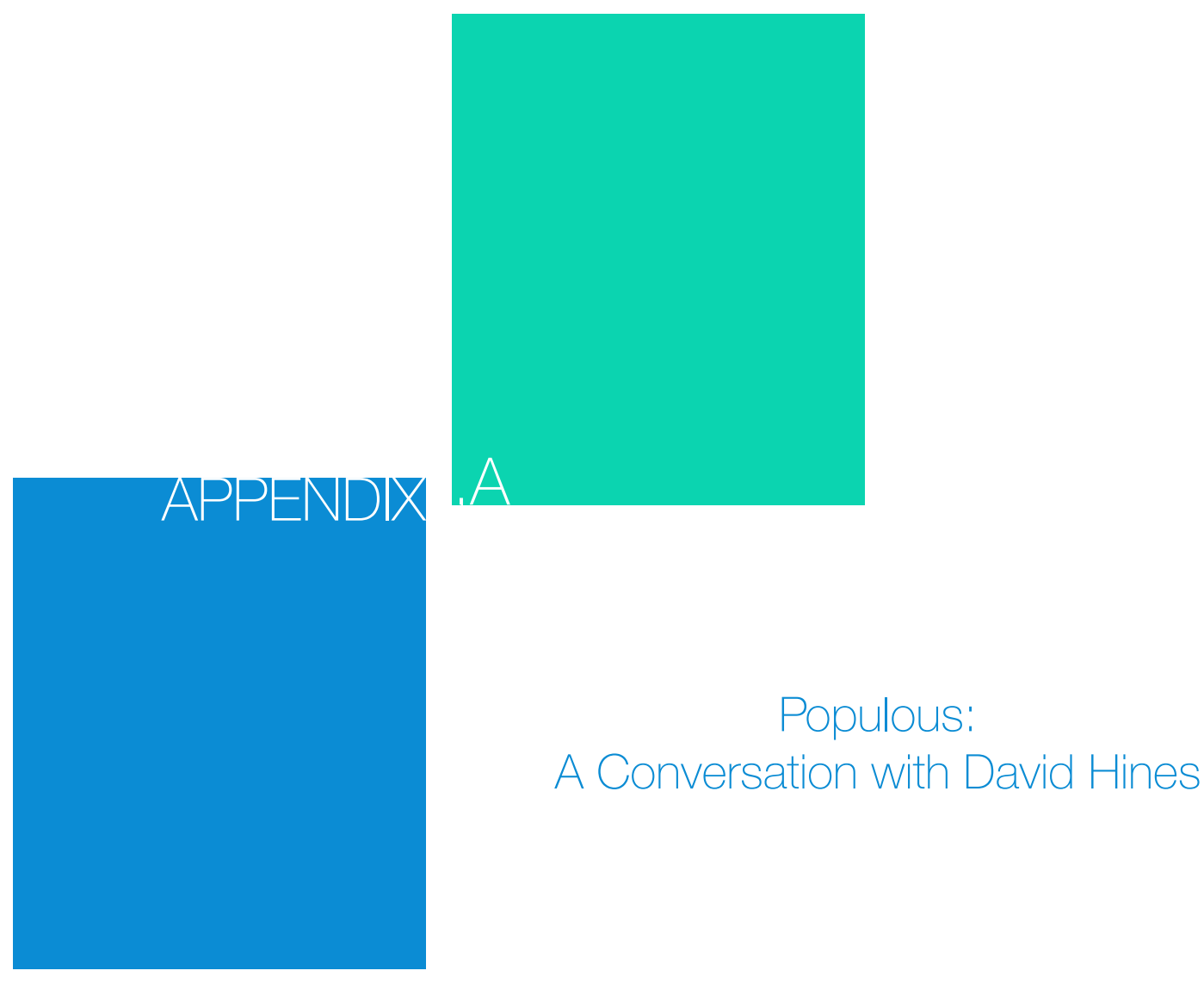


"Sport in the UK differed from sports growth in America because the urban grade of the field is already situated there in the urban fabric [of the city]."

_David Hines 
In December 2014 I had the opportunity to

sit down for a discussion with David Hines, Associate

Principle at the London offices of Populous.

David works in a leadership role at the forefront of applying parametric modeling in Rhino and Grasshopper to developing stadia projects, both in design development and construction methods.

Most notably, David was the project architect of the Aviva National Rugby Stadium in Dublin, Ireland. This project was one of the first ever developed at this scale using a parametric approach, and applications included developable form, seating optimization and sunlight analysis. He later expanded on this working method acting as a project leader on Fischt Stadium for the Sochi Olympics.

Following is our discussion.

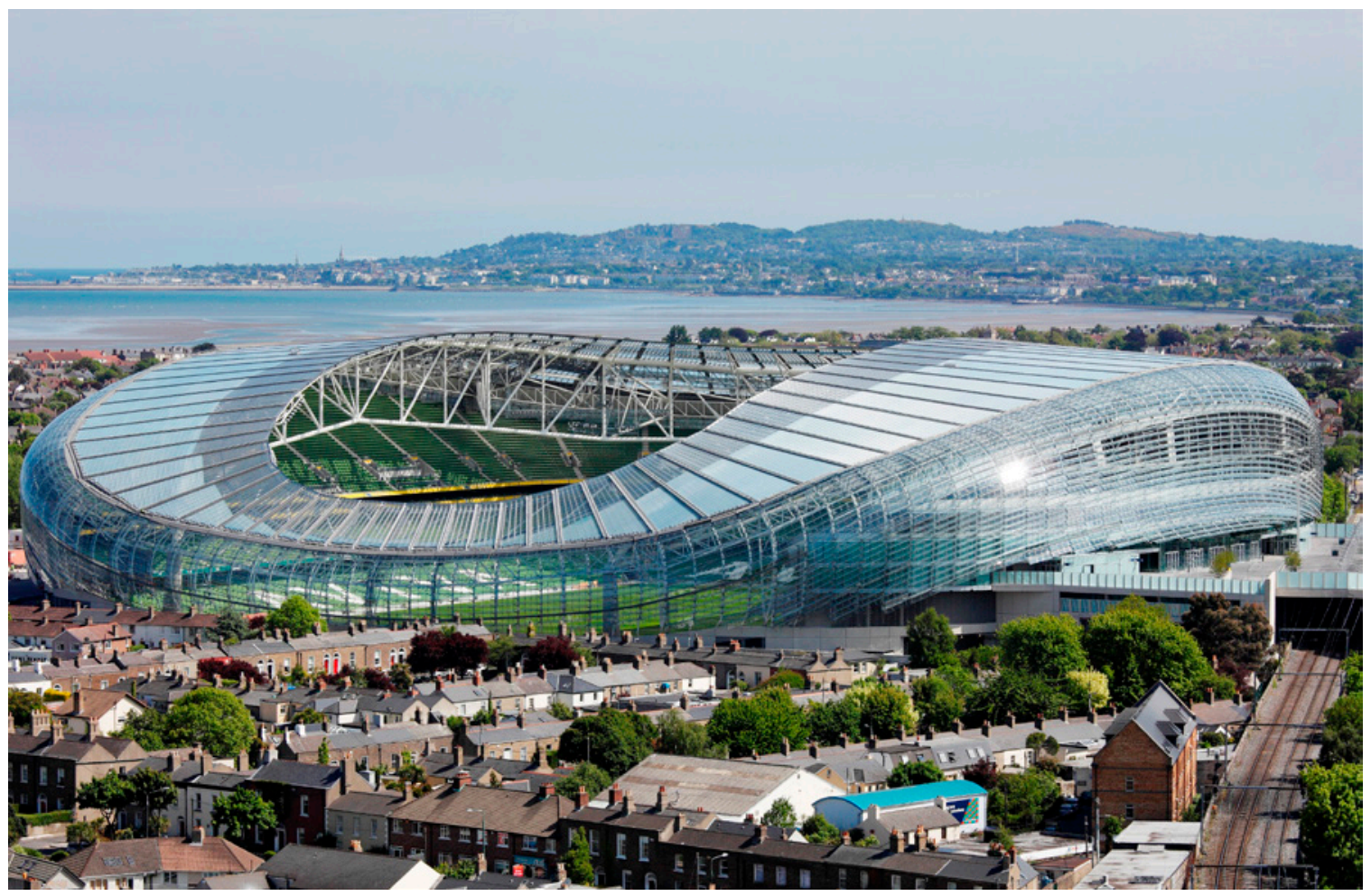


BC: You're an Associate Principle at the Populous London

office, and you also lead much of the digital work coming from the studio. Let's start with what your role was in the new national rugby/football stadium for Ireland, now known as the Aviva Stadium in Dublin.

DH: I was running the package form of the building, the roof structure, and the cladding, more of the architectural side of it. We were using generative components at the time. We approached Roly Hudson [previously of the University of Bath and Dalhousie University] and said come on and give us a hand with this job, and we ended up establishing quite a good working relationship, so he's helped us out with a few other jobs as well.

BC: So it seems that you drove much of the overall form for Aviva, and collaborated with Roly to establish the relationship between the formal complexity and the parametric support. I'm curious how you came to that form, and specifically how you used parametric software to develop the formal relationship to the existing surrounding buildings. The way that we saw it [in visiting the site], I don't think there are very many large stadium projects that are similarly surrounded by small townhomes. They looked like they were probably 50-100 years old, easily within 25 meters of the structure. It seems the complete opposite of most North American stadiums.

$\mathrm{DH}$ : For the starting point, the reasoning behind it was that the site was very historic; it was the ground of the first 
international rugby game ever played at Lansdowne Road.

They wanted to keep the site at that location. Admittedly

the old stadium got ripped down and the existing field was

completely removed, so they rebuilt it and the orientation

of the field shifted about eighteen degrees. But the site

was always known as Lansdowne Road. So in that regard,

the point about the North American stadiums, because of

its heritage, its history, they were keen to keep it where it

was. Also, in Europe it is far more common as stadiums

are developed through more urban fields that they get

swallowed up by housing and other developments, so that

over time they have sort of grown out of the land that

they are located. You can look at any number of English

Premiership football clubs, and you will see they are

generally in the suburb that is associated with their name

[Chelsea, Fulham, West Ham, Tottenham]. So sports in

the UK differed from sports growth in America because

the urban grade of the field is already situated there in the

urban fabric.

BC: And this falls directly in line with the low number of car parking spaces available at Aviva Stadium; you couldn't

build parking spaces because of the tight site conditions, so you leveraged the strategy to this constraint?

DH: Yes, there are very few cars at Aviva Stadium, there's probably only car parking space for about 50-60 cars under the stadium, and everyone else, basically $95 \%$ of everyone comes by foot or by the DART station. The stadium was limited to 50,000 capacity because of the size, the actual 
width of Lansdowne Road, you couldn't' safely get people away from the stadium in sufficient time to have any greater capacity at that location. So the old stadium, when it was taken down, is pretty much the same capacity as the new stadium, except the new stadium is all seated.

$\mathrm{BC}$ : So with a dearth of parking but requiring the same capacities for the program, what was the approach to the way Aviva deals with the traffic flows of large crowds?

DH: In Europe we use a document called the Green Guide, also known as Safety in Sports Grounds. In this green guide there are calculations that give you things like a movement ratio, of division, and generally what you do, a sort of rule of thumb is that if you had 10,000 people for example, and you need to move them out of the building in 8 minutes, which is a sort of regulation time to get someone to a safe area, you take the 10,000 and divide it by 8 [time in minutes], and the you divide that number by 66 [the given rate of flow for people descending a staircase] and that should give you the relative width of space you need for those people to descend a staircases. That would give you something around $18 \mathrm{~m}$ wide. So if you have a stadium of 30,000 people, you need 3 inputs of $18 \mathrm{~m}$ to make sure they can all get away from that building in a safe amount of time. That's the flow rate they will move to. That's the speed that people descend stairs. I think if you divide by 82 that's the rate that people will move for people walking flat. But generally you use the stair one because are generally coming out of these buildings down the fire stair, so they're

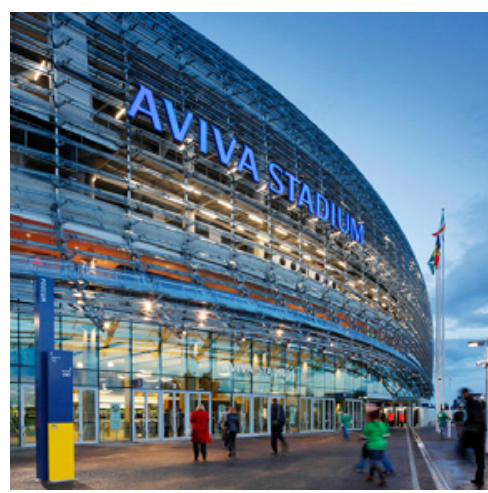


already into that ratio of movement. And you don't want

crowds to crush and slow down when they hit the stair.

BC: And part of it is how you incorporated the DART system into something as big as a 50,000 seat stadium. In Canada I think there are three stadiums in the entire country that actually have some kind of rapid transit service like that [Toronto's Air Canada Center/Rogers Center, Montreal's Bell Center, Vancouver's BC Place/ Rogers Arena); otherwise they seem to use bussing, which is typically quite slow into the suburbs, or just cars as a single-service mode of transport. I'm curious what the approach was to incorporating a DART system into the overall scheme.

$\mathrm{DH}$ : We had to understand the capacities of what the DART can load people on and also capacities that it could take people, and then we worked with the train operators to regulate how many people we could move through the station safely on a match day. Everybody else basically walks, and then the station was designed in accordance with how those flows can safely get on and off the platform around game time. Also we had to put an underpass below the Stadium side, across the North side of the Lansdowne Road. That underpass by the stadium allows the crowd flow to move down the Lansdowne Road when the [road/ train] barriers are down and the trains are coming. So on a non-match day, there's little barriers that are raised as normal, because it's a level crossing, then on a match day they keep the barriers down full-time and regulate that so 
no one runs across the level crossing. And they just open the gates underneath the stadium so the crowd flow can just shift through the underside of the stadium itself. So you basically divert the pedestrians through tunnels and on to the other side of Lansdowne Road. No traffic runs down that road on match days because everyone in the area knows it's a game day, so they don't try to drive in that area. It just fills with people. You'd have to be totally foreign to the area to even try to drive down Lansdowne Road on a match day, because the police would turn you away early on. The crowd flow would stop you from moving anywhere.

BC: You would never get anywhere close to the stadium in a car in the first place.

DH: Right. For these stadiums, you make a scenario and engineer a scenario with regards to the movement of people, for fire regulations, for transport flows, etc. We work with companies who do traffic analysis and crowdflow analysis, and they run a program called "Legion" to create a crowd-flow model which they can input the CAD data. They can include people en masse and have them move from points A-to-B. The people will have a slight bit of chaos theory, and it basically replicates what a crowd will do. You can push people through a building or through a street, see how they bottleneck and see how quickly they move and get out. We use these companies to make the simulation models for these big buildings. That's how we address it. 
BC: We've been talking about this as a critical schematic

criteria; did this play into the form for the Aviva Stadium at all?

$\mathrm{DH}$ : We won the Aviva stadium on a competitive submittal competition. One other company was pitching an idea to the client but they preferred our design. We sold the building on the fact that it was purely on the rights of light. That allowed you to dip the building on the North side because of those historical houses on the Havelock Square to the North. The actual area that the stadium is in is one of the most affluent areas of Dublin; they're all very expensive. Because it's close to the center, with traditional Victorian houses, they're either big homes or very expensive houses divided up into flats or small offices in that area. It was critical that we slot the stadium in and not upset too many neighbors. We really couldn't purchase houses and just extend the whole building. We had to fit it into the land. With this we had to look at sun path studies, then we did initial modeling and designs and ran these through the sunpath analysis tools. We did lots of early modeling in Rhino, just shape modeling what would work generally with the shadow on the North side. That was where we started the process of modeling this form of the building curves, and then defining, really before we started any parametric work, the radius of the side of the building, the radius around the curve of the edge and the roof element.

When we put this design together it was before any big parametric tools had been on the market. We really took 
the planning application stage based on form modeling in

Rhino, and then post-planning we then moved the model of the building into generative components and brought Roly [Hudson] on board specifically to translate the 3D geometry into a parametric tool that we could then work with and apply cladding to. We realized that it would be futile and incredibly laborious to try and design a cladding system and then not have a parametric tool where we would have to reapply if the shape of the building changed. So it was really a whole parametric process driven out of a necessity for what we had to produce in the time frame we had. We ended up tying together the engineers with us, so we used the same shared parametric model and the engineers would then structurally model from that for the skin-model cladding. The two were tied to the same baseline geometry, and that was quite critical because it meant we had a shared modeling environment, the same setup of modeling. And that allowed us to basically progress the design over about a year with the structural engineers. We developed the cladding systems and they in turn would be developing the structural bend and movement systems. We adapted that base model about nine times throughout the year, tweaking the roof slightly, tweaking the facades, cushioning the building envelope.

The building was kind of straightened on the roof, around the edge and then a straight curve on the façade; we used that element and then we would roll out the building around the grid, and then move the control points of the start and stop with each of those three bits of geometry to 
get that form. When you strip the building down, it is all

very rational arcs to the pieces of the building in straight

lines, even though the apparent shape has a double curve.

Everything is rationalized and stripped down so you can

dissect it and construct the pieces. Because we always have

to think in advance about how you would manufacture any

mullion or any transom or any fixing position, we always

had to dissect how you could rationalize it on a grid, on a

single arc radius, because you can't really effectively and

efficiently bend mullions in splines. They just become

entrapped.

BC: So for a double-curve you basically had to have two separate curves moving on separate flat planes, rather than actually having double-curved units? That's where you get the apparent double-curve without any actual doublecurve?

DH: Well you can do double-curved elements but the price just goes through the roof per piece. And the manufacturing process is just that much more difficult. We made very conscious decisions that nothing was ever a double-curve; it was only ever curved in a single plane. And nothing was ever of a spline-nature, like an ellipse or a complex shape; everything was an arc or a straight line. Because if you give a steel contractor a spline [variablychanging curve], they will rationalize it down to a series of arcs they can bend on a machine. They will take a curved object and just cut it into pieces and then they'll know how many bends they'll extract to get the piece. Again, that's

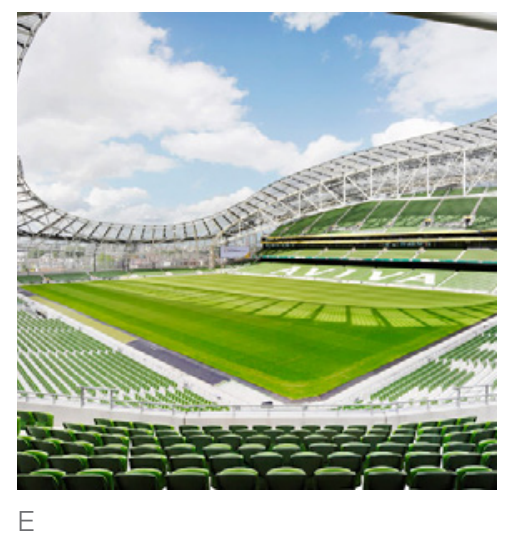


still more expensive than having a single, known arc. So we

made all of the façade mullions that you see in the majority of the building to have the same radius. They're all about 110 meter radius. And then only the bit that differs is the arc of the mullion at which makes the connection between the roof and the façade. That has a different radius of each one. So basically four-fifths of your building has the same radius. Then we know the panels sit on straight axles, they work when it's a double-arm rotation element.

BC: Right, and we could see this plainly at the stadium. There were a couple points where it almost comes down to about head-height, maybe 2.5 meters above the ground. From all of the images you see of the stadium, the skin seems to look like a singular unit, but it wasn't until we were up at the third floor inside the stadium where, if you get close enough, you can start to see how they've all shifted just subtly enough to let the air through. And that was one of the key orientation methods I believe, where the rain would clearly run down from the next panel to the next, etc. without a true wall system in place?

DH: That's correct. We designed the fixing piece to the mullions, it had a neck piece, and then there is a v-plate that had the rotation, so everything was pre-modeled and pre-drilled and pre-defined so that they couldn't get it wrong on site. Then you had a bar so that each panel was on an axial bar, and that panel itself was then in an elevation, so it was a fixed thing, but at the same point the holding piece, the key piece at the end, you have a 
double-arm axial piece, and that was locked in. But the way that blade was fixed to that axial was always on a slightly different rotation, so that you could then gradually see the moment of opening and closing.

BC: But it's not working in a motor system of any sort?

DH: No, they're all fixed. We ran models to find the opening, and then fixed them, so everything was all manufactured, the off-site assembly was basically all done and defined in a position for everything. Everything was pre-drilled, everything was pre-made. Nothing was ever measured or set-out on site, except for the major erection of the mullion pieces. All the racks were affixed and drilled, all the panels were made in order so they knew the installation sequence. Because you could never eyeball that sort of system. All of that was worked out. In-house we designed the panels so that it acts like a rain-screen. The folds in the panel give it rigidity, so it has rigidity over its length. If it were flat it would wobble about too much. So we deliberately bent edges to it so that the rain would blow down the pieces. The axle piece that went around the mullion, that bit was always the same piece. We designed only one casting mold that made all those bits. They were drilled onto the mullion at the position in the factory, and then the holes were drilled onto the face, and that bit was placed on the front. It only had one locking location.

BC: And then that was all brought back into the main structural components that were put up as the very first 
thing on site? Other than that, everything was unitized into

a single piece and the only variation was in the connections to the structure itself?

$\mathrm{DH}$ : You mean to get the elevation of the blade?

BC: Yes I think so.

$\mathrm{DH}$ : The elevation of the blade was prescribed in the factory because the actual axle would have the blades fixed onto it. We knew the bar was always located at one point, in the factory they had a jig that they would make the blade pieces and the axle pieces. So they could be in a completely different rotation. They could number them in sequence,

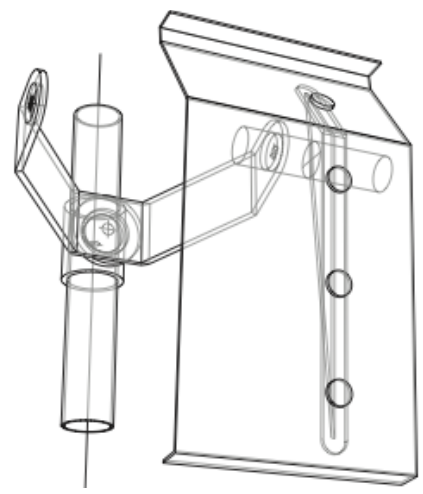
store them, and then as they erected them they had a logic going top-down on the building with each blade. Also what you don't really see is that the panels themselves do change width, very slightly. If you peel an orange, it's going to get wider at its middle and then thinner at the end. It's the same geometry. The panels in the middle were slightly wider and then they get thinner. We had over 4,400 panels, and we rationalized this down to $30 \mathrm{~mm}$ increments in panel widths. This rationalized it down to about 52 different versions with different widths. Because the panels never touch, you don't really see that. The panels are anything from about 3.8 to $4.2 \mathrm{~m}$, so a jump of $30 \mathrm{~mm}$ on the ends can't really be perceived."

BC: Then I suppose to extend this, it seems like the Aviva Stadium in Dublin and Fisht Stadium in Sochi are 
distinctly related, at least compared to the other more

prominent projects in the Populous portfolio; perhaps

that's not true but it certainly appears so.

DH: [Laughing] That might be because I worked on both.

BC: Right, I assumed that might also have something to do with it [laughs].

DH: I mean, I do get asked this a lot, why parametric and why all those specific elements. I do get asked how you design parametrically, etc. A lot of it just comes down to - how and what are you designing, and then how do you materialize it? So we embarked on a vehicle without thinking, 'Oh, we're going to do this parametrically. We just embarked on the design, and what's possible today, and what can we model, and what can we build. And that's why even when we were moving into Rhino shapes we made quick rules. No splines, no double-curved surfaces. Let's just rationalize this down, let's see if we can actually manufacture this. So we were always thinking about the production of line. We used the parametric tools to make our lives bearable, because we realized quickly we had so much re-work to do if we changed the shape. You couldn't do that traditionally; it would just be a nightmare. It would take years to ever re-draw anything. And so really it came from design, and it came from the site, and it came from the rights to light, and then we made it as beautiful as we could. It was polycarbonate because it's an open-air environment. It needed to be transparent and it needed 
to reflect some daylight. It the best material that we could ever justify in that close proximity to the houses. It materialized through that design process, and then we used the computer to build it.

Fisht stadium was based on the mountains in the region, and those two hoods point towards the center of the Olympic Park. From inside the stadium the idea is that from the views and the vistas you can see the mountains; they replicate the forms of the boomerang-hoods that give the shape of those roofs. There's a reference to the known stadiums that people like. When it came to the cladding, we did it parametrically because we shifted very late in the design from an overlapping shingle system to set tiles of ETFE to reduce the weight of the roof cladding, and we did it with this pattern to replicate the shingle effect. It just so happens that, again, the right way, the quickest way to do it was modeling in $3 \mathrm{D}$ and driving a parametric pattern so that you got the staggered effect, you got that shingled appearance, and it worked quite nicely. If it was all one material it would just look typical. The structural ribs below run in the opposite direction; it would just look like a series of ribs, but now it looks like a pattern.

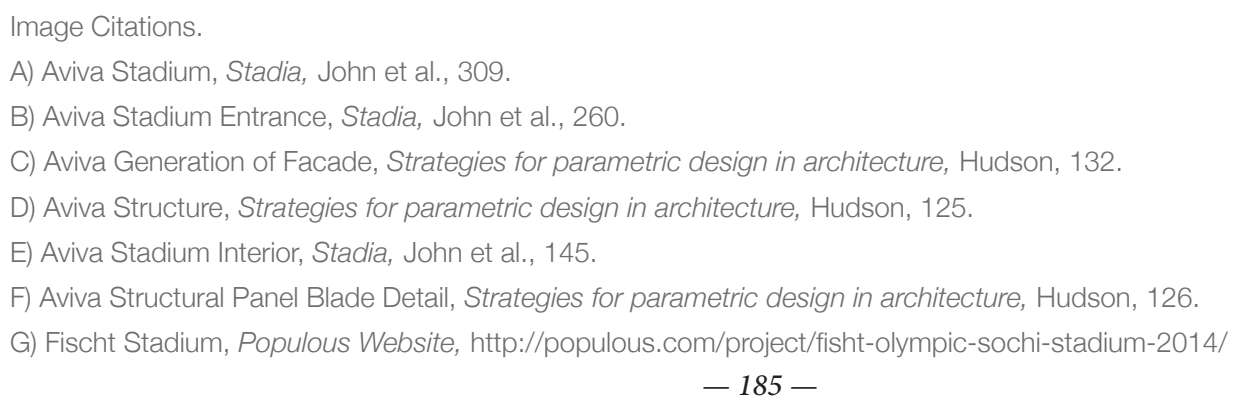




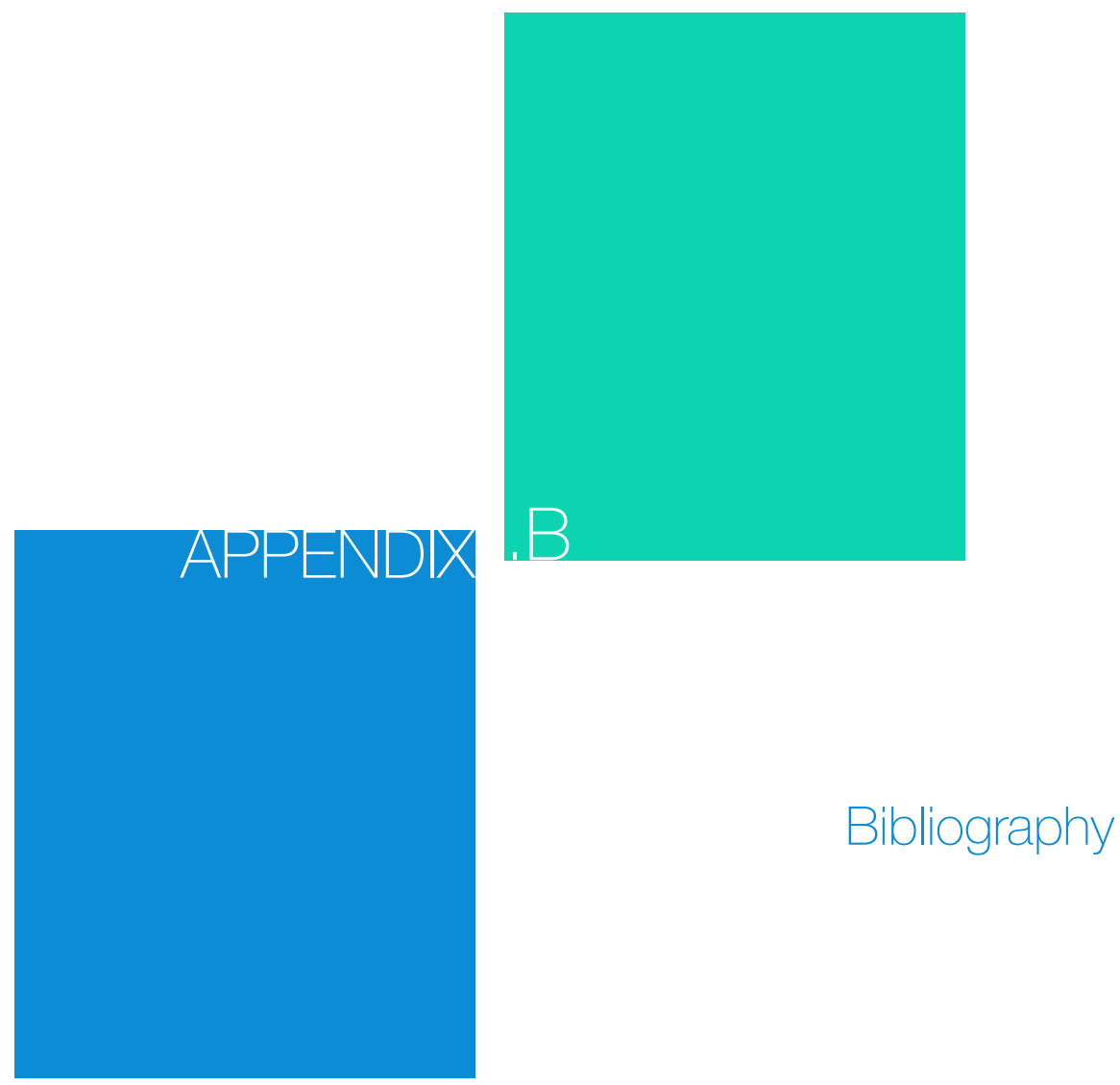


Aalto Digital Design Laboratory. “ADD Discourse.” ADD Summary (2013): 23-38.

Alexander, Christopher. "The Question of Computers in Design." Landscape 14, Issue 3 (1963): 6-8.

Allen, Stan. Points \& Lines: Diagrams and Projects for the City. New York: Princeton Architectural Press, 1999.

Anderse, Michael Asgaard and Jeanne Rank Schelde. "Architecture Gives Shape to Our Existence." In New Nordic: Architecture \& Identity, 32-55. Denmark, Rosendahls, 2012.

Carpo, Mario. "Breaking The Curve." Artforum International 02 (2014): 169-173.

Carr, Nicholas. The Glass Cage: Automation and Us. New York: W. W. North \& Co., 2014.

Central Intelligence Agency. "Canada." The World Factbook. https://www.cia.gov/ library/publications/theworld-factbook/geos/ca.html.

Coates, Paul. "The Deep Structure of the Picturesque." Architectural Design 231 (2014): 32-37.

Curtis, William J. R. Modern Architecture Since 1900, $3^{\text {rd }}$ Ed. New York: Phaidon, 2010.

Derix, Christian. "The Space of People in Computation." Architectural Design 231 (2014): 14-23.

Fjeld, Per Olaf. Sverre Fehn: The Pattern of Thought. New York: Monicelli Press, 2009.

Frampton, Kenneth. Modern Architecture: A Critical History, 4th Ed. United Kingdom: Thames \& Hudson, 2007.

Gabarro, Gustavo Garcia. "Nature in the Sagrada Familia." In Sagradda Familia: Gaudi’s Opus Magnum, 68101. Spain: Media Minds SL.

Hagan, Susannah. Digitalia: Architecture and the Digital, the Environmental and the Avant-Garde. United Kingdom: Routledge, 2008.

Hvattum, Mari. "Making Place." In New Nordic: 
Architecture \& Identity, 100-115. Denmark:

Rosendahls, 2012.

Ingels, Bjarke. "WorldCraft." Guest Lecture at Harvard

Graduate School of Design, Boston, MA, 4

September 2014.

International Union of Railways. "General Definitions of

High Speed." About High Speed. 28 July 2014. http://

www.uic.org/spip.php?article971.

John, Geraint, Rod Sheard and Ben Vickery. Stadia: The

Populous Design and Development Guide, $5^{\text {th }} \mathrm{Ed}$.

New York: Routledge, 2013.

Kinoshita, Gene. “The Ottawa Train Station.” Guest Lecture

at Carleton Azrieli School of Architecture, Ottawa,

ON, 18 March 2015.

Kjeldsen, Kjeld. Foreword to New Nordic: Architecture \& Identity, 10-15. Denmark: Rosendahls, 2012.

Leatherbarrow, David. "Building In and Out of Place." Architectural Design 85 Vol. 2 (2015): 24-29.

Lynch, Kevin. The Image of the City. USA: MIT Press, 1960.

Mitchell, William J. Foreword to New Tectonics: Towards

a New Theory of Digital Architecture, edited by Yu-Ting Liu and Chor-Kheng Lim, 10-18. Taiwan:

Birkhauser, 2009.

Pallasmaa, Juhani. The Embodied Image. Italy: John Wiley \& Sons, 2011.

Pallasmaa, Juhani. The Eyes of the Skin. UK: John Wiley \& Sons, 2012.

Pallasmaa, Juhani. The Thinking Hand. Italy: John Wiley \& Sons, 2009.

Patt, Trevor. "Taipei 2.0.2: Computation and the Urban Generic.” In Highrise Shuffle, 111-119. Finland: Multiprint Oy, 2011.

Quantrill, Malcolm. Plain Modern: The Architecture of Brian MacKay-Lyons. New York: Princeton Architectural Press, 2005.

Rush, Fred. On Architecture. New York: Routledge, 2009. 
Rutten, David. "Worrisome Trends in Architecture

Education." I Eat Bugs for Breakfast Blog. 9 August

2013. https://ieatbugsforbreakfast.wordpress.com

/2013/08/09/worrisome-trends-in-architecture-

education/.

Schumacher, Patrik. "The Autopoiesis of Architecture.”

Lecture presented at the Architectural Association, London, United Kingdom, December 2010.

Simons, Andrew. Interview with author, 12 November 2014.

Snohetta. Conditions. Germany: Lars Muller Publishers, 2007.

Snohetta. Works. Germany: Lars Muller Publishers, 2009.

Sorkin, Michael. "Critical Measure: Why Criticism Matters." Architectural Review 1408 (2014): 91-99.

Teer, Marc. "Requirements to Work in the Top 50

Architecture Firms.” Black Spectacles. 27 June 2013.

http://blackspectacles.com/blog/software-licensurerequirements-to-work-top-50-architecture-firms\#.

VK1ZhSf31o4.

Timme, Elizabeth. Archinect Sessions Podcast 11. Interview

by Paul Petrunia et al. Archinect. Los Angeles, 22 January 2015.

UNESCO World Heritage Centre. Operational Guidelines

for the Implementation of the World Heritage

Convention. July 2012. http://whc.unesco.org/

archive/opguide12-en.pdf.

Venturi, Robert. Complexity and Contradiction in Architecture. New York: Baronet Litho Inc., 2007.

Vidler, Anthony. Warped Space: Art, Architecture, and Anxiety in Modern Culture. Boston: MIT Press, 2000.

Whiteson, Leon. Modern Canadian Architecture. Canada: Hurtig Publishers, 1983.

Woodbury, Robert. Elements of Parametric Design. New York: Routledge, 2010. 


$$
\text { 承 }
$$

